\title{
Chemical Analysis Results for Potable Water from ISS Expeditions 21 through 25
}

\author{
John E. Straub II ${ }^{1}$, Debrah K. Plumlee ${ }^{2}$, and John R. Schultz ${ }^{3}$ \\ Wyle Integrated Science \& Engineering Group, Houston, Texas, 77058 \\ J. Torin $\mathrm{McCoy}^{4}$ \\ NASA Johnson Space Center, Houston, Texas, 77058
}

\begin{abstract}
The Johnson Space Center Water and Food Analytical Laboratory (WAFAL) performed detailed groundbased analyses of archival water samples for verification of the chemical quality of the International Space Station (ISS) potable water supplies for Expeditions 21 through 25 . Over a 14-month period the Space Shuttle visited the ISS on four occasions to complete construction and deliver supplies. The onboard supplies of potable water available for consumption by the Expeditions 21 to 25 crews consisted of Russian groundsupplied potable water, Russian potable water regenerated from humidity condensate, and US potable water recovered from urine distillate and condensate. Chemical archival water samples that were collected with U.S. hardware during Expeditions 21 to 25 were returned on Shuttle flights STS-129 (ULF3), STS-130 (20A), STS-131 (19A), and STS-132 (ULF4), as well as on Soyuz flights 19-23. This paper reports the analytical results for these returned potable water archival samples and their compliance with ISS water quality
\end{abstract} standards.

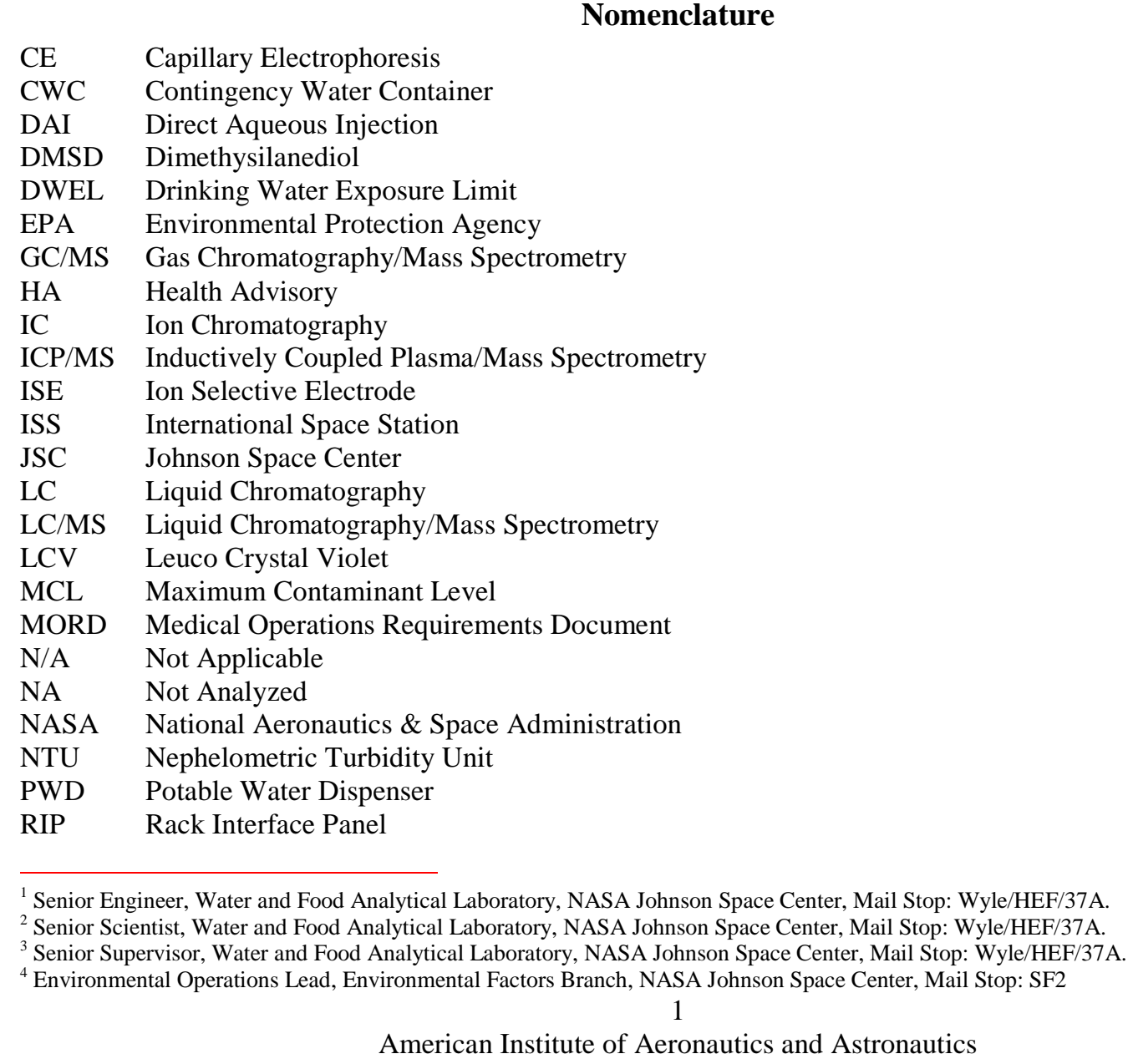




$\begin{array}{ll}\text { SM } & \text { Service Module } \\ \text { SRV-K } & \text { System for Regeneration of Condensate Water } \\ \text { SVO-ZV } & \text { System for Water Storage and Dispensing } \\ \text { SWEG } & \text { Spacecraft Water Exposure Guideline } \\ \text { TDS } & \text { Total Dissolved Solids } \\ \text { THM } & \text { Trihalomethanes } \\ \text { TOCA } & \text { Total Organic Carbon Analyzer } \\ \text { U.S. } & \text { United States } \\ \text { UPA } & \text { Urine Processor Assembly } \\ \text { UV } & \text { Ultraviolet } \\ \text { WAFAL } & \text { Water and Food Analytical Laboratory } \\ \text { WPA } & \text { Water Processor Assembly } \\ \text { WRS } & \text { Water Recovery System }\end{array}$

\section{Introduction}

While the International Space Station (ISS) recently celebrated the 10-year anniversary of human occupation by some 25 different expedition crews, the Johnson Space Center's (JSC) Water and Food Analytical Laboratory (WAFAL) quietly continued its key role of performing ground-based chemical analyses of returned archival water samples to verify that the ISS potable water remained safe for crew consumption. Chemical analysis results for samples collected during Expeditions 1-20 have been previously published. ${ }^{1-8}$ This paper presents and discusses the analytical results from chemical analyses of the archival potable water samples collected and returned during Expeditions 21 - 25, as detailed in Table 1. Only chemical analysis results are discussed herein, as the JSC Microbiology Laboratory has responsibility for microbiological monitoring of the ISS potable water supplies.

The WAFAL sent a representative to the Kennedy Space Center in Florida to retrieve and return the ISS potable water samples that returned on Shuttle flights STS-129 (ULF3), STS-130 (20A), STS-131 (19A), and STS32(ULF4). These samples were packed into coolers along with ice packs and transported to Houston via commercial air transport. The ISS water samples that returned on Soyuz 19 through Soyuz 23 were retrieved from the vehicle by Russian specialists and subsequently transferred to a NASA representative for cold packing and transportation to Houston via NASA jet. The WAFAL representative took possession of the return after their arrival in Houston for delivery to the JSC laboratory.

Once the samples arrived in the WAFAL, allocation was performed based upon return sample volume. The samples collected into U.S. 1-L Teflon ${ }^{\circledR}$ sample bags typically contained sufficient sample volume (> $\left.500 \mathrm{~mL}\right)$ to support full chemical characterization using the standard and custom analytical methods identified in Table 2.

Water samples collected for return on Soyuz were typically less than $500 \mathrm{~mL}$ volume, requiring elimination of some analyses and/or reductions in sensitivity of other analyses performed. During data analysis and reporting, the analytical results for each sample are compared as appropriate against either the Russian Segment potable water quality requirements found in the ISS Medical Operations Requirement Document (MORD) ${ }^{9}$ or the U.S. Segment potable water quality requirements found in the System Specification for the ISS document. ${ }^{10}$

\section{Background}

Over the 14-month period beginning October 2009, the Expeditions 21 to 25 crews onboard the International Space Station had access to 3 different sources of potable water: U.S. potable water recovered from urine distillate and humidity condensate, Russian ground-supplied potable water, and Russian potable water regenerated from humidity condensate. These different water supplies were available via water systems located in the U.S. Segment and the Russian Segment of the ISS.

\section{U.S. Segment Water Recovery System}

The U.S. Segment Water Recovery System (WRS) was delivered on STS-126 (ULF2) in November of 2008 and began processing a combined wastewater feed consisting of both urine distillate and humidity condensate. During an initial checkout period of about 90-days the product water was tested to verify quality and was subsequently approved for crew consumption beginning in May 2009 prior to the start of ISS 6-person crew operations. 
Table 1. Summary of Water Samples Collected \& Received during Expeditions 21 through 25

\begin{tabular}{|c|c|c|c|c|c|}
\hline Expedition & Flight No. & $\begin{array}{l}\text { Samples } \\
\text { Received } \\
\end{array}$ & Sample Type & Sample Collection Date & $\begin{array}{c}\text { Sample Receipt } \\
\text { Date }\end{array}$ \\
\hline \multirow{10}{*}{21} & STS-129 (ULF3) & 1 & PWD Ambient & \multirow[t]{4}{*}{$11 / 10 / 2009$} & \multirow[t]{4}{*}{$11 / 30 / 2009$} \\
\hline & & 1 & PWD Hot & & \\
\hline & & 1 & SVO-ZV & & \\
\hline & & 1 & SRV-K Hot & & \\
\hline & Subtotal: & 4 & & & \\
\hline & Soyuz 19 & 1 & PWD Ambient & \multirow[t]{3}{*}{$10 / 20 / 2009$} & \multirow[t]{3}{*}{$12 / 14 / 2009$} \\
\hline & & 1 & PWD Hot & & \\
\hline & & 1 & SVO-ZV & & \\
\hline & Subtotal: & 3 & & & \\
\hline & Total: & 7 & & & \\
\hline \multirow{10}{*}{22} & STS-130 (20A) & 1 & PWD Ambient & $1 / 6 / 2010$ & \multirow[t]{4}{*}{$2 / 22 / 2010$} \\
\hline & & 2 & SVO-ZV & $1 / 6 / 2010,2 / 3 / 2010$ & \\
\hline & & 1 & SRV-K Hot & $2 / 3 / 2010$ & \\
\hline & & 1 & SRV-K Warm & $1 / 6 / 2010$ & \\
\hline & Subtotal: & 5 & & & \\
\hline & \begin{tabular}{|l} 
Soyuz 20 \\
\end{tabular} & 1 & SVO-ZV & \multirow[t]{3}{*}{$3 / 3 / 2010$} & \multirow[t]{3}{*}{$3 / 26 / 2010$} \\
\hline & & 1 & SRV-K Hot & & \\
\hline & & 1 & SRV-K Warm & & \\
\hline & Subtotal: & 3 & & & \\
\hline & Total: & 8 & & & \\
\hline \multirow{15}{*}{23} & STS-131 (19A) & 1 & PWD Ambient & \multirow[t]{2}{*}{$3 / 3 / 2010$} & \multirow[t]{4}{*}{$4 / 22 / 2010$} \\
\hline & & 1 & PWD Hot & & \\
\hline & & 1 & PWD Aux Port & \multirow[t]{2}{*}{$2 / 25 / 2010$} & \\
\hline & & 1 & WPA RIP & & \\
\hline & Subtotal: & 4 & & & \\
\hline & STS-132 (ULF4) & 2 & PWD Ambient & $4 / 26 / 2010,5 / 18 / 2010$ & \multirow[t]{4}{*}{$5 / 27 / 2010$} \\
\hline & & 2 & SVO-ZV & $4 / 26 / 2010,5 / 18 / 2010$ & \\
\hline & & 1 & SRV-K Hot & $5 / 18 / 2010$ & \\
\hline & & 2 & SRV-K Warm & $4 / 26 / 2010,5 / 18 / 2010$ & \\
\hline & Subtotal: & 7 & & & \\
\hline & Soyuz 21 & 1 & PWD Hot & \multirow[t]{3}{*}{$3 / 31 / 2010$} & \multirow[t]{3}{*}{$6 / 3 / 2010$} \\
\hline & & 1 & SVO-ZV & & \\
\hline & & 1 & SRV-K Hot & & \\
\hline & Subtotal: & 3 & & & \\
\hline & Total: & 14 & & & \\
\hline \multirow{4}{*}{24} & Soyuz 22 & 2 & PWD Ambient & 7/14/2010, 9/15/2010 & \multirow[t]{3}{*}{$9 / 26 / 2010$} \\
\hline & & 1 & PWD Hot & $8 / 25 / 2010$ & \\
\hline & & 1 & WPA RIP & $7 / 29 / 2010$ & \\
\hline & Total: & 4 & & & \\
\hline \multirow{3}{*}{25} & Soyuz 23 & 2 & PWD Hot & $10 / 19 / 2010,11 / 23 / 2010$ & \multirow[t]{3}{*}{$11 / 29 / 10$} \\
\hline & & 1 & SRV-K Warm & $11 / 23 / 10$ & \\
\hline & Total: & 3 & & & \\
\hline
\end{tabular}

The WRS includes a urine processor assembly (UPA) that uses a distillation process to purify pretreated urine. The resulting urine distillate is stored in a wastewater tank where it is combined with humidity condensate recovered from the ISS atmosphere. The WRS also includes a water processor assembly (WPA) that uses adsorption/ion exchange and thermal catalytic oxidation to remove contaminants from the combined wastewater feed stream. After iodine biocide is added, the WPA product water is then stored for delivery to the potable water bus. The potable water dispenser (PWD) receives water directly from the potable bus and provides either hot or ambient water for crew use, after removing the iodine biocide at the point of use. Iodinated potable water transferred from the Space Shuttle can also be added directly to the WPA storage tank in the event that make-up water is needed.

A total organic carbon analyzer (TOCA) was also delivered with the WRS in November of $2008 .{ }^{11}$ Since that time the TOCA has served as a real-time tool for monitoring the total organic carbon (TOC) content of the WPA product water on a weekly basis. The TOCA includes a dedicated hose for direct sampling of water from the WPA product tank. It has also been used monthly to analyze samples collected from the PWD use-point in Teflon ${ }^{\circledR}$ sample bags. In-flight TOCA results provide insight into the total amount of organic contamination in the WPA water without identification of specific organic constituents. 
Table 2. Water Analytical Methods

\begin{tabular}{|c|c|}
\hline Parameter & Method \\
\hline pH \& conductivity & Potentiometric \\
\hline Total Dissolved Solids & Gravimetric \\
\hline Turbidity & Nephelometric \\
\hline Iodine \& iodide & Leuco crystal violet (LCV) \\
\hline Fluoride & Ion chromatography (IC) \\
\hline Metals/Minerals & Inductively coupled plasma/mass spectrometry (ICP/MS) \\
\hline Inorganic anions \& cations & Ultraviolet or heated persulfate oxidation \\
\hline Total organic carbon (TOC) & GC/MS with a purge \& trap concentrator \\
\hline Alcohols \& glycols & GC/MS after liquid/liquid extraction \\
\hline Volatile organics & Capillary electrophoresis (CE) \\
\hline Semi-volatile organics & Liquid chromatography (LC) with UV diode array detector \\
\hline Organic acids \& amines & GC/MS after derivatization \& extraction \\
\hline Urea/Caprolactam & LC/MS/MS \\
\hline Formaldehyde & Direct injection GC/MS or LC with refractive index detector \\
\hline Glycerol &
\end{tabular}

\section{Russian Segment Water Systems}

The Russian condensate water recovery system (SRV-K), which is located in the Service Module (SM), treats humidity condensate recovered from SM cabin air into potable water as previously described. ${ }^{1-3}$ Condensate from the U.S. Segment that has been stored in a Contingency Water Container (CWC) can also be transferred using a Condensate Feed Unit and processed by the SRV-K. After the condensate is filtered to remove particulate matter, it flows through a catalytic filter reactor, phase separator, and multifiltration beds to remove organic and inorganic contaminants. Prior to storage the processed water flows through a conditioning bed where silver biocide is added, as well as minerals (calcium, magnesium, and fluoride) to improve palatability. The conditioned product water is pumped from storage to the SRV-K galley where it is pasteurized then made available to the crews via two dispenser ports (hot and warm). Should the demand for potable water exceed the availability of condensate for processing, the crews can install a container of stored potable water to a connection located downstream of the conditioning bed and upstream of the galley for use as make-up water.

The Russian Segment stored potable water system or SVO-ZV is also located in the Service Module as previously described. ${ }^{1-3}$ The SVO-ZV provides the crew access to Russian ground-supplied potable water (Rodnik water) that has been launched on Russian Progress vehicles and delivered to the ISS. During pre-launch preparation, groundwater from Korolev, Russia is filtered and silver is added electrolytically as a biocide before storage on Progress in 210-liter Rodnik tanks.

\section{Discussion of Analytical Results}

Results from chemical analyses of the SRV-K (regenerated), SVO-ZV (stored), and U.S. WPA archival potable water samples collected during Expeditions 21 through 25 are summarized in Appendices 1, 2 and 3, respectively. Specification limits, if any, are included in these data tabulations for comparison with the analytical results. A discussion of findings obtained from the chemical analysis of archival samples collected during each expedition and their compliance with ISS requirements follows.

\section{EXPEDITION 21}

A total of 7 chemical archival potable water samples, including 1 SRV-K hot, 2 SVO-ZV, 2 PWD hot, and 2 PWD ambient, were collected during Expedition 21 as detailed in Table 1. The 4 samples collected on November 10, 2009 were returned on STS-129 (ULF3) and received in the WAFAL on November 30, 2009. The 3 samples collected on October 20, 2009 were returned on Soyuz 19 and received in the WAFAL on December 14, 2009. All of the samples were collected using U.S. 1-liter Teflon ${ }^{\circledR}$ water sample bags. The 4 samples that were returned on Shuttle all had sufficient sample volume to support full chemical characterization. Due to limited sample volume, turbidity was not analyzed on the PWD hot sample and solids were not analyzed on any of the 3 samples collected for Soyuz return. 
ISS US SEGMENT:

WPA Processed Water Samples

All chemical parameters measured for the 4 PWD samples were within limits specified in Table LXX of SSP $41000 .{ }^{10}$ Nickel levels ranged from 17 to $33 \mathrm{ug} / \mathrm{L}$ (see Figure 1 ). The total iodine (I) was $<0.05 \mathrm{mg} / \mathrm{L}$ and meets the point of consumption limit of $0.2 \mathrm{mg} / \mathrm{L}$ total I (see Figure 2). The iron levels have continued to be at low levels after the PWD disinfection flush on 3/23/09 (see Figure 3). The TOC values ranged from 0.14 to $0.30 \mathrm{mg} / \mathrm{L}$ and are well within the $3 \mathrm{mg} / \mathrm{L}$ limit (see Figure 4). Trace levels of methyl sulfone (34 to $111 \mu \mathrm{g} / \mathrm{L}$ ) and formaldehyde ( $<2$ to 2 $\mu \mathrm{g} / \mathrm{L}$ ) were identified; however, these levels were toxicologically insignificant.

\section{ISS RUSSIAN SEGMENT:}

SRV-K Potable Water Sample

All chemical parameters measured for the SRV-K potable water sample were within the requirements found in Table D-1 of the ISS MORD, Revision C. ${ }^{9}$ The nickel level of $34 \mu \mathrm{g} / \mathrm{L}$ was well below the limit of $100 \mu \mathrm{g} / \mathrm{L}$ (see Figure 5). The silver biocide level of $14 \mu \mathrm{g} / \mathrm{L}$ was low, indicating that heating of the water by the pasteurization unit continues to be the main source of microbial control in the SRV-K galley. As shown in Figure 6, an updated plot of the TOC trend in the SRV-K water samples, the TOC level was $0.17 \mathrm{mg} / \mathrm{L}$ and well below the specification limit. Trace levels of acetone $(4 \mu \mathrm{g} / \mathrm{L})$ chloroform $(0.7 \mu \mathrm{g} / \mathrm{L})$, diethyl ether $(4 \mu \mathrm{g} / \mathrm{L})$, acetaldehyde $(3 \mu \mathrm{g} / \mathrm{L})$, dibutylphthalate $(8 \mu \mathrm{g} / \mathrm{L})$, and N-phenyl-2-naphthylamine $(4 \mu \mathrm{g} / \mathrm{L})$ were identified. Their levels, however, were toxicologically insignificant.

\section{SVO-ZV Potable Water Samples}

All chemical parameters measured for the $2 \mathrm{SVO}-\mathrm{ZV}$ water samples were within the ISS MORD requirements except for a turbidity level of 1.6 NTU in the October 20, 2009 sample, which slightly exceeded the 1.5 NTU limit. The concern with elevated turbidity in the SVO-ZV samples is that particulates causing the turbidity can shield bacteria from the silver biocide. The dissolved silver level of $81 \mu \mathrm{g} / \mathrm{L}$ in the October 20 sample indicates that a small amount of the particulates are due to colloidal silver which may help to mitigate this concern. The total silver levels of 28 to $111 \mu \mathrm{g} / \mathrm{L}$ continued to be lower than the expected range of 400 to $500 \mu \mathrm{g} / \mathrm{L}$, thereby increasing the risk of microbial growth in the water. Updated plots of manganese, and of turbidity, total and colloidal silver $\left(\mathrm{Ag}_{\text {colloidal }}=\right.$ $\left.\mathrm{Ag}_{\text {total }}-\mathrm{Ag}_{\text {dissolved}}\right)$, and formate in SVO-ZV samples are presented in Figures 7 and 8. The TOC levels in the 2 SVO-ZV samples were $0.36 \mathrm{mg} / \mathrm{L}$ and $2.70 \mathrm{mg} / \mathrm{L}$, well below the $20 \mathrm{mg} / \mathrm{L}$ limit. Low levels of chloroform (1.6 to $51.9 \mu \mathrm{g} / \mathrm{L})$ and a trace level of bromodichloromethane $(1.9 \mu \mathrm{g} / \mathrm{L})$ were identified; however, these levels were not considered to be toxicologically significant.

\section{EXPEDITION 22}

A total of 8 chemical archival potable water samples, including 2 SRV-K hot, 2 SRV-K warm, 3 SVO-ZV, and 1 PWD ambient, were collected during Expedition 22 as detailed in Table 1. The 5 samples collected on January 6, 2010 and February 3, 2010 were returned on STS-130 (20A) and received in the WAFAL on February 22, 2010. The 3 samples collected on March 3, 2010 were returned on Soyuz 20 and received in the WAFAL on March 26, 2010. All of the samples were collected in U.S. 1-liter Teflon ${ }^{\circledR}$ water sample bags. The 5 samples that were returned on Shuttle all had sufficient sample volume to support full chemical characterization. Due to limited sample volume, solids were not analyzed on the 3 samples that were returned on Soyuz.

\section{ISS US SEGMENT:}

\section{WPA Processed Water Sample}

All chemical parameters measured for the PWD ambient sample were within limits specified in SSP 41000 . The nickel level was $10 \mu \mathrm{g} / \mathrm{L}$ (refer to Figure 1). The total iodine (I) level was $<0.05 \mathrm{mg} / \mathrm{L}$ and met the point of consumption limit of $0.2 \mathrm{mg} / \mathrm{L}$ (Figure 2). The iron level continued to be at a low level after the PWD disinfection flush on 3/23/09 (Figure 3). The TOC value was $0.16 \mathrm{mg} / \mathrm{L}$ and is well within the $3 \mathrm{mg} / \mathrm{L}$ limit (see Figure 4). Trace levels of methyl sulfone $(34 \mu \mathrm{g} / \mathrm{L})$ and formaldehyde $(4 \mu \mathrm{g} / \mathrm{L})$ were identified; however, these levels were toxicologically insignificant.

\section{ISS RUSSIAN SEGMENT:}

\section{SRV-K Potable Water Samples}

All chemical parameters measured for the 4 SRV-K warm \& hot potable water samples were within the ISS MORD requirements. The nickel levels $(26-48 \mu \mathrm{g} / \mathrm{L}$ ) were within specifications (Figure 5). The silver level (added 

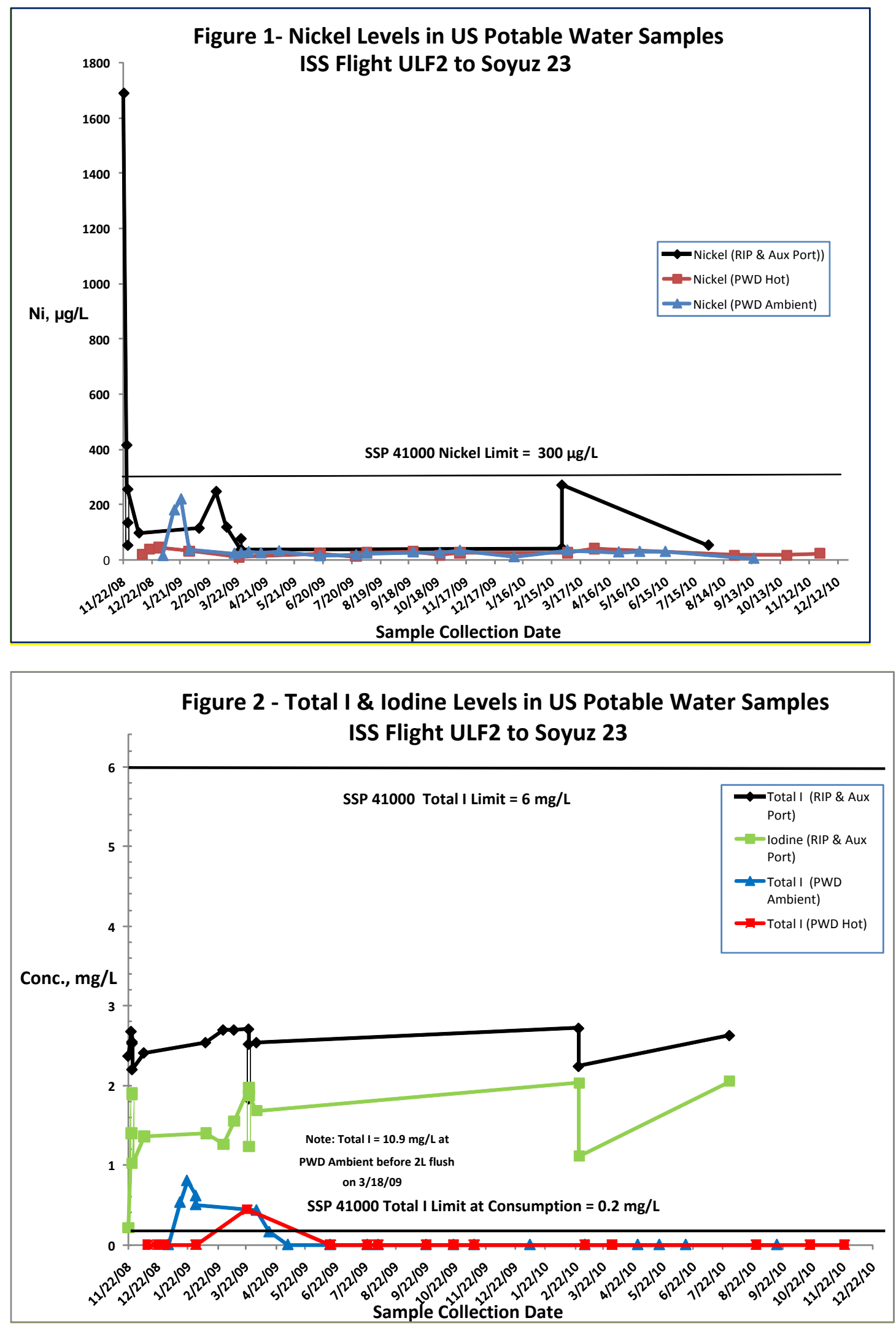

6

American Institute of Aeronautics and Astronautics 

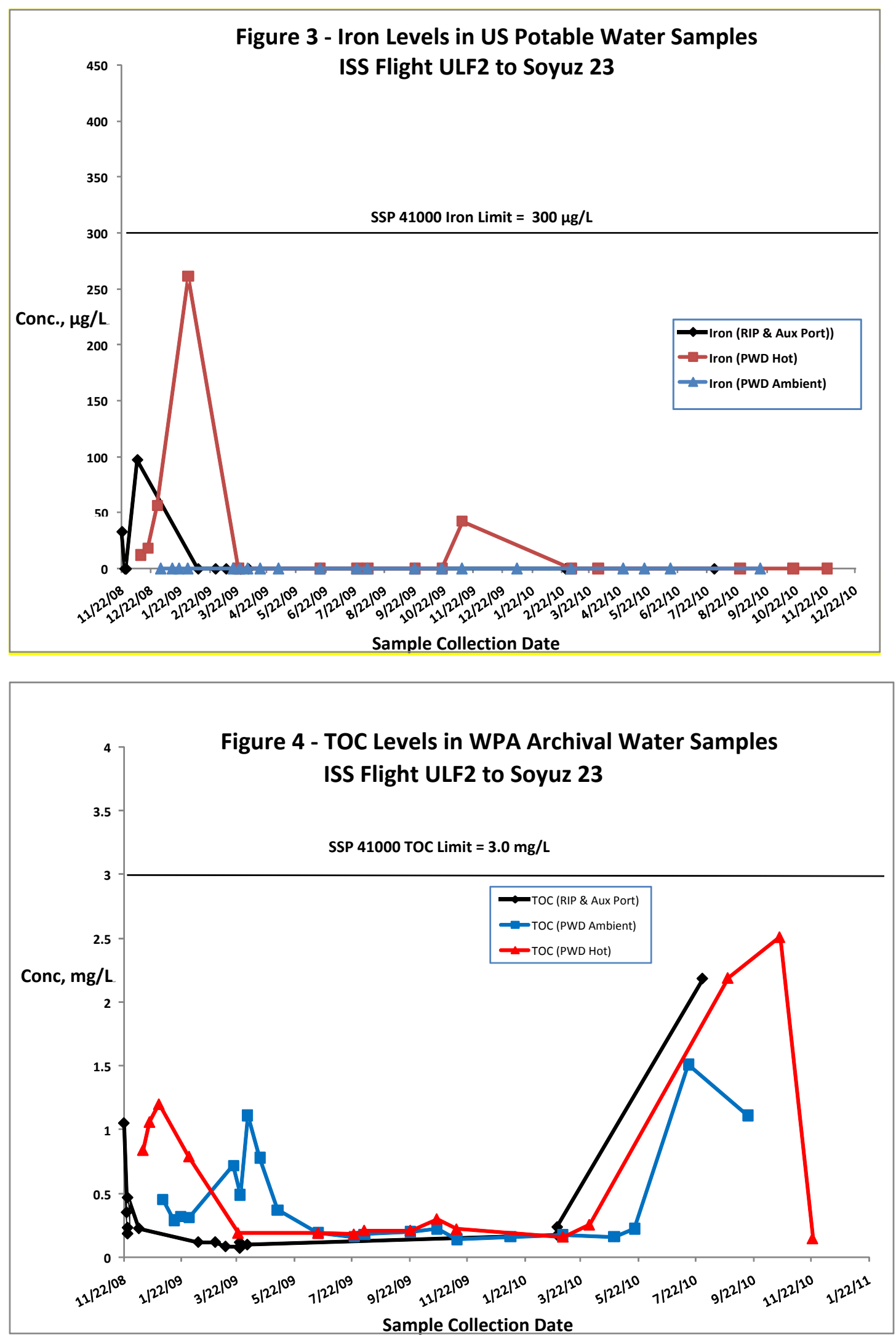

7

American Institute of Aeronautics and Astronautics 
Figure 5 - Nickel Levels in SRV-K Water Samples

ISS Flight 4A to Soyuz 23

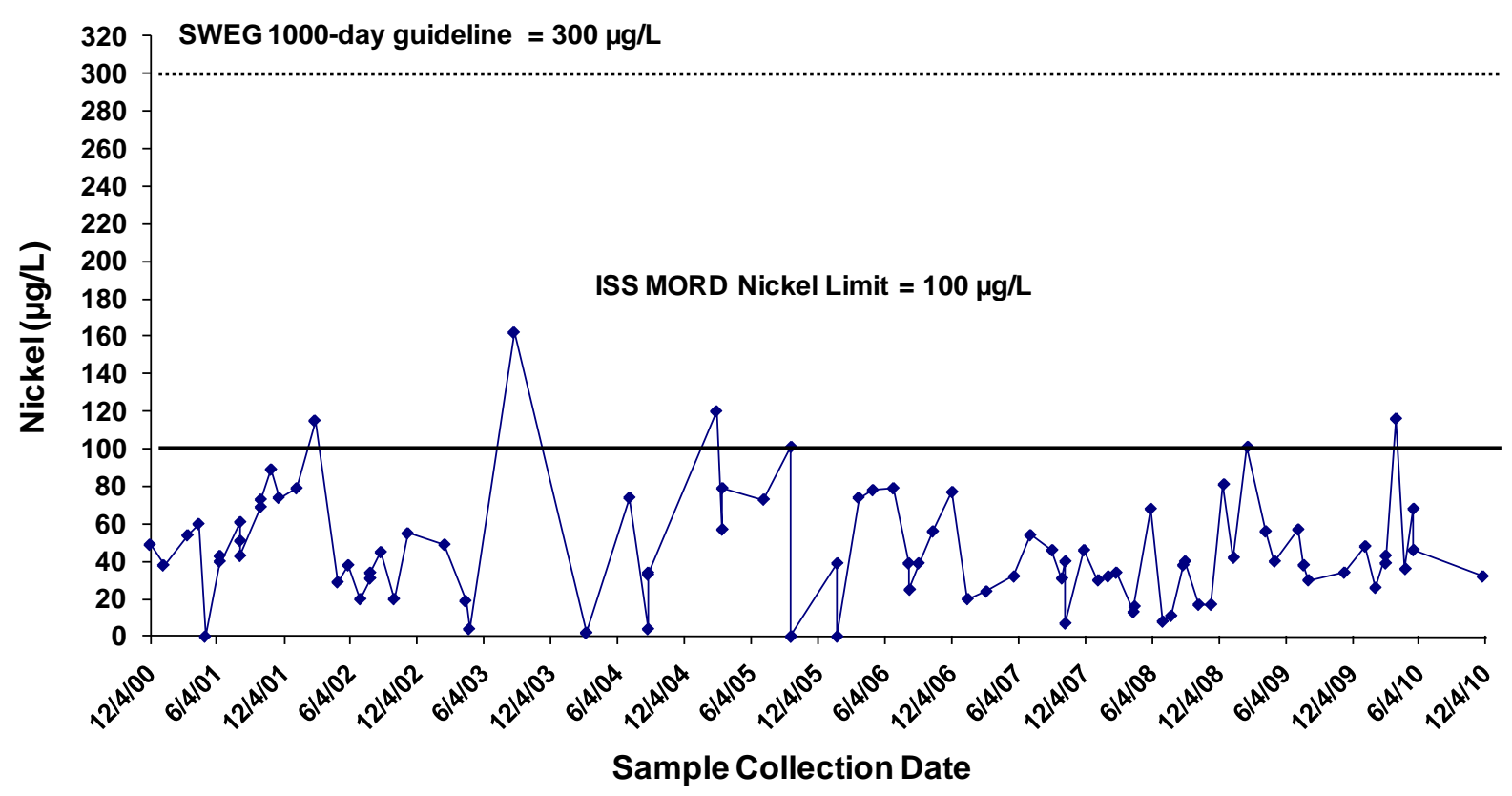

Figure 6 - Total, Formate, \& Non-formate Organic Carbon in SRV-K Potable Water ISS Flight 4A to Soyuz 23

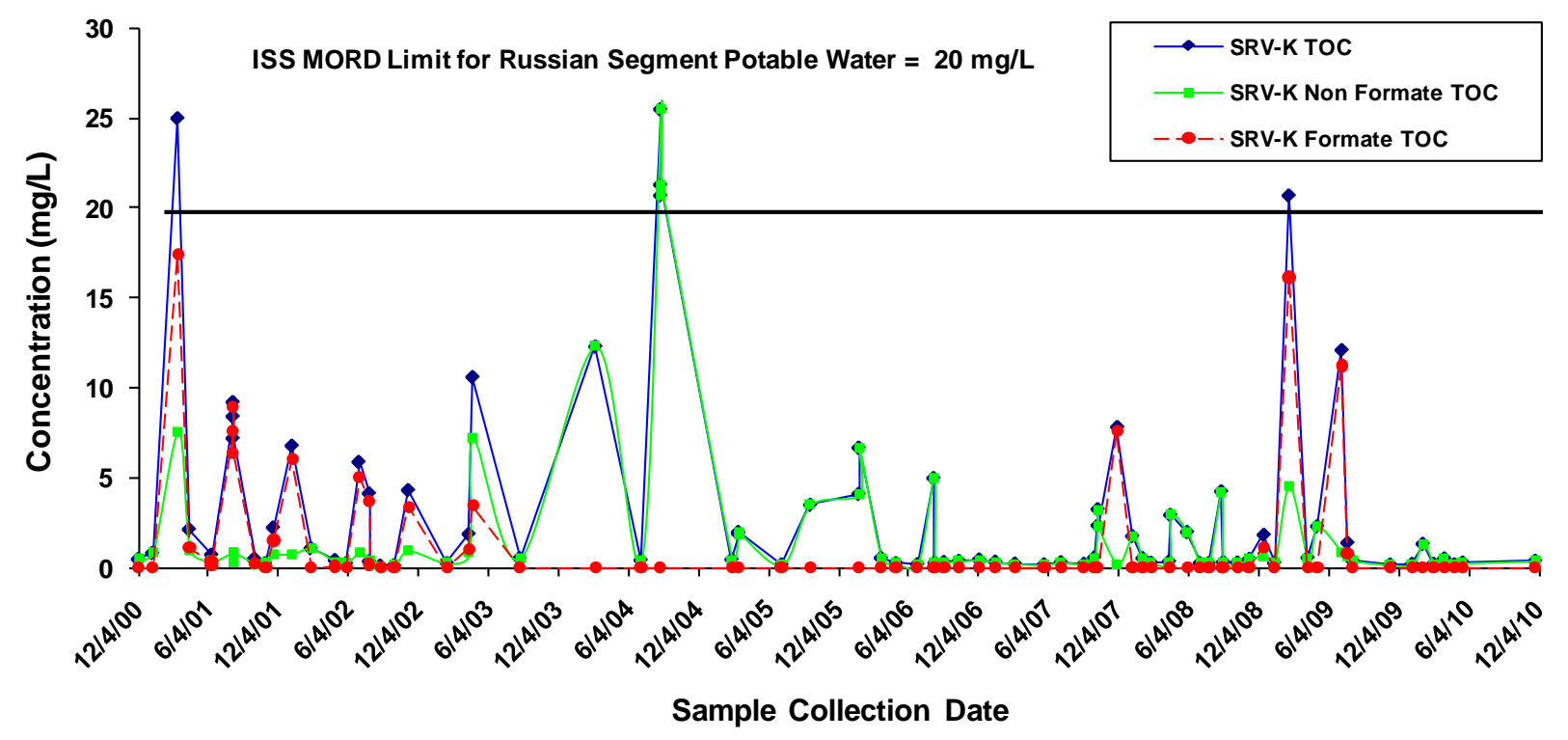

8

American Institute of Aeronautics and Astronautics 
Figure 7 - Manganese Levels in SVO-ZV Water Samples ISS Flight 5A to Soyuz 21

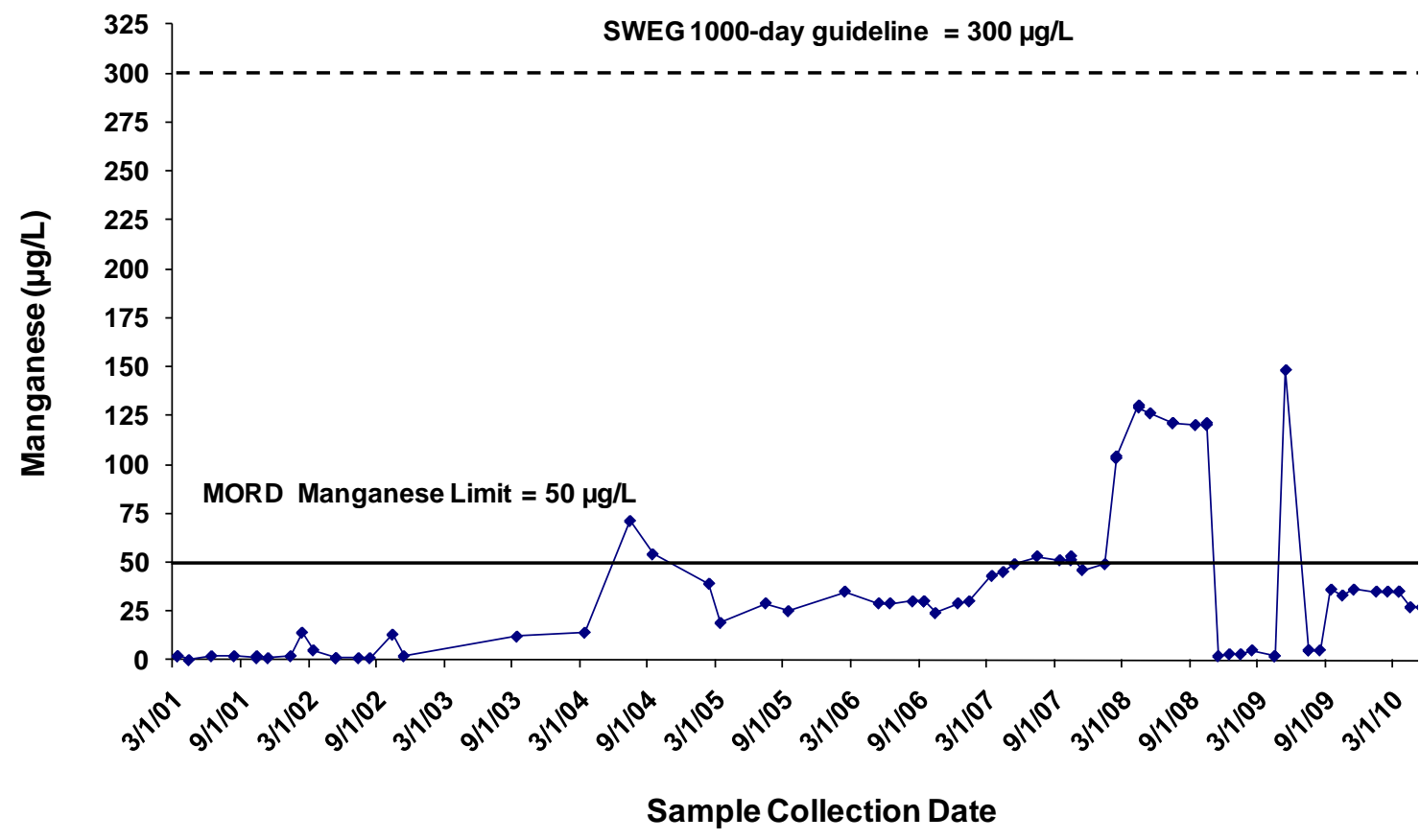

Figure 8 - Turbidity, Formate, Total \& Colloidal Silver in SVO-ZV Water Samples ISS Flight 5A to Soyuz 21

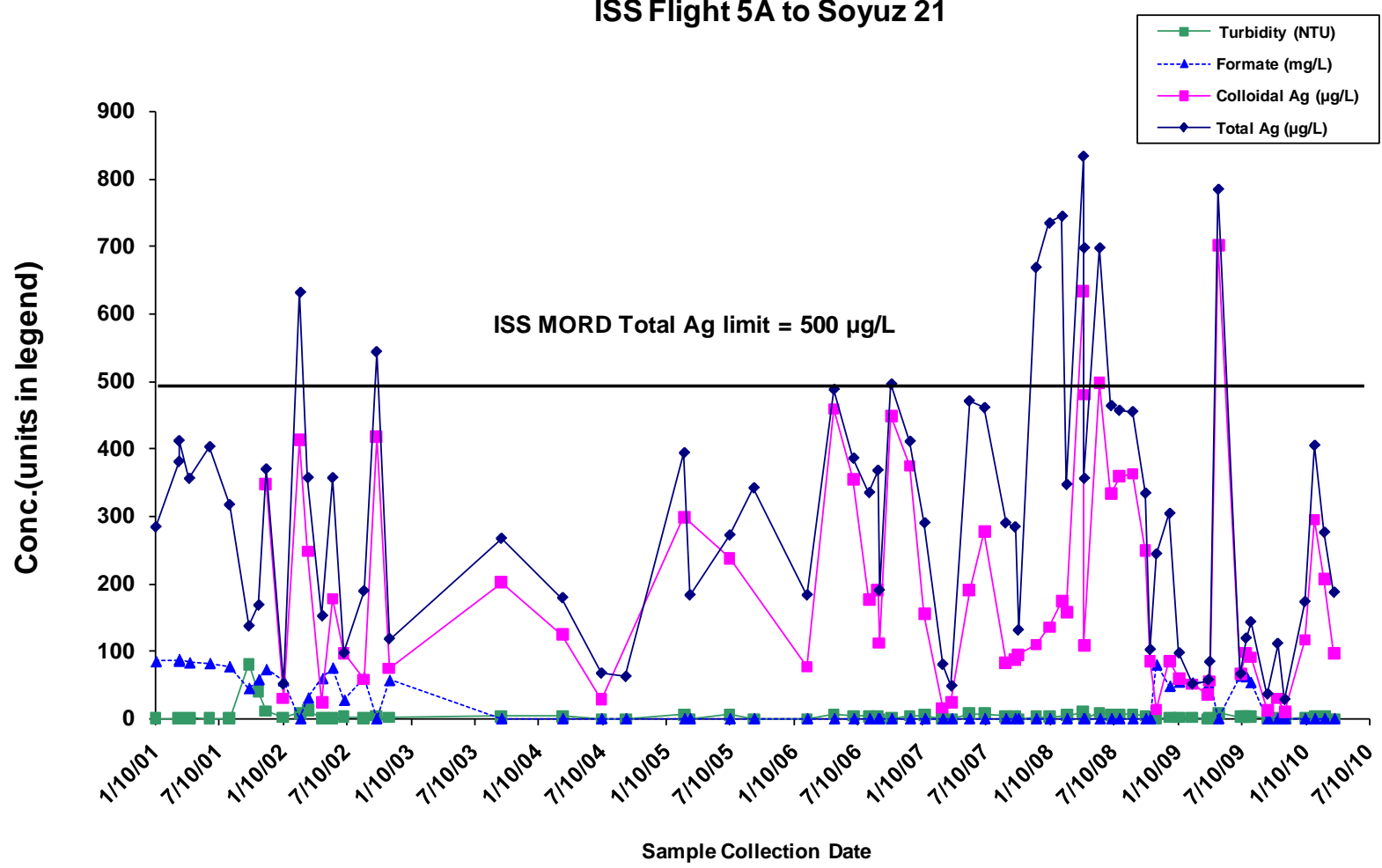


biocide) continued to be low in the SRV-K samples (2-90 $\mu \mathrm{g} / \mathrm{L})$, indicating that heating of the water by the galley continues to be the main source of microbial control. The TOC levels ranged from 0.19-1.32 mg/L (Figure 6). Trace levels of bromodichloromethane $(1.2 \mu \mathrm{g} / \mathrm{L}), 1,2$-dichloroethane $(0.6 \mu \mathrm{g} / \mathrm{L})$, chloroform $(28 \mu \mathrm{g} / \mathrm{L})$, diethyl ether $(4$ $\mu \mathrm{g} / \mathrm{L})$, o-xylene $(1.5 \mu \mathrm{g} / \mathrm{L})$, and dibutylphthalate $(5 \mu \mathrm{g} / \mathrm{L})$ were identified; however, these levels were not considered to be toxicologically significant.

\section{SVO-ZV Potable Water Samples}

All chemical parameters measured for the $3 \mathrm{SVO}-\mathrm{ZV}$ water samples were within the ISS MORD requirements except for turbidity (2.0 - 3.8 NTU) which exceeded the 1.5 NTU limit. The concern with elevated turbidity in the SVO-ZV samples is that particulates causing the turbidity can shield bacteria from the silver biocide. The dissolved silver levels ranged from 2-90 $\mu \mathrm{g} / \mathrm{L}$ (Figure 8) indicating that a small amount of the particulates are due to colloidal silver which may mitigate this concern. The total silver levels in the January 6 and March 3 samples of 173 and 276 $\mu \mathrm{g} / \mathrm{L}$, respectively, (Figure 8) were both below the expected range of 400-500 $\mu \mathrm{g} / \mathrm{L}$, increasing the risk of microbial growth in the water. On the other hand, the total silver level in the February 3 sample was $405 \mu \mathrm{g} / \mathrm{L}$ and within the expected range. Manganese levels in the 3 samples ranged from 35-36 $\mu \mathrm{g} / \mathrm{L}$ and were well below the ISS MORD limit (Figure 7). The TOC levels ranged from 3.26-3.48 mg/L, all well within specifications. Although chloroform $(89.2 \mu \mathrm{g} / \mathrm{L})$ exceeded the $80 \mu \mathrm{g} / \mathrm{L}$ EPA limit in the March $3^{\text {rd }}$ sample, it was within the 6,500 $\mu \mathrm{g} / \mathrm{L}$ Spacecraft Water Exposure Guideline (SWEG) for total trihalomethanes and thus did not pose a crew health risk. Only trace levels of bromodichloromethane (3.0-3.1 $\mu \mathrm{g} / \mathrm{L})$ and chloroform $(68-77 \mu \mathrm{g} / \mathrm{L})$ were identified in the other 2 samples.

\section{EXPEDITION 23}

A total of 14 chemical archival potable water samples, including 2 SRV-K hot, 2 SRV-K warm, 3 SVO-ZV, 3 PWD ambient, 2 PWD hot, 1 PWD Auxiliary Port, and 1 WPA Rack Interface Panel (RIP) were collected during Expedition 23 as detailed in Table 1. The 4 samples collected on February 25, 2010 and March 3, 2010 were returned on STS-131 (19A) and received in the WAFAL on April 22, 2010. The 7 samples collected on April 26, 2010 and May 18, 2010 were returned on STS-132 (ULF4) and received in the WAFAL on May 27, 2010. The 3 samples collected on March 31, 2010 were returned on Soyuz 21 and received in the WAFAL on June 3, 2010. All of the samples were collected using U.S. 1-liter Teflon ${ }^{\circledR}$ water sample bags. The 5 samples that were returned on Shuttle all had sufficient sample volume to support full chemical characterization. Due to limited sample volume, solids were not analyzed on any of the 3 samples collected for Soyuz return.

\section{ISS US SEGMENT:}

\section{WPA Processed Water Samples}

All chemical parameters measured for the 7 potable water samples taken from the PWD, WPA RIP, and PWD Auxiliary Port met requirements in SSP 41000. The nickel levels (see Figure 1) ranged from 26-270 $\mu \mathrm{g} / \mathrm{L}$, with the highest level coming from the Aux port. The total iodine (I) was $<0.05 \mathrm{mg} / \mathrm{L}$ for the PWD ambient and hot samples and meets the requirement limit of $0.2 \mathrm{mg} / \mathrm{L}$ at the points of consumption (Figure 2). Total iodine levels were 2.24 and $2.72 \mathrm{mg} / \mathrm{L}$ in the PWD Aux and WPA RIP samples, both within the range of 2-4 mg/L that is the desired level for WPA product water. Iron was not detected in any of the 7 samples. The TOC levels ranged from 0.16-0.26 mg/L, well within the $3 \mathrm{mg} / \mathrm{L}$ limit (see Figure 4). Trace levels of acetone (11-39 $\mu \mathrm{g} / \mathrm{L})$, iodomethane $(5 \mu \mathrm{g} / \mathrm{L})$, methyl sulfone $(34-98 \mu \mathrm{g} / \mathrm{L})$, o-xylene $(1.5 \mu \mathrm{g} / \mathrm{L})$ and formaldehyde $(2-6 \mu \mathrm{g} / \mathrm{L})$ were identified in the samples. These trace levels were toxicologically insignificant.

\section{ISS RUSSIAN SEGMENT:}

\section{SRV-K Potable Water Samples}

All chemical parameters measured for the 4 SRV-K water samples met requirements listed in the ISS MORD with the exception of the nickel level of $116 \mu \mathrm{g} / \mathrm{L}$ in the March 31, 2010 SRV-K hot sample, which slightly exceeded the MORD specification of $100 \mu \mathrm{g} / \mathrm{L}$, but was well within the established SWEG of $300 \mu \mathrm{g} / \mathrm{L}$ (Figure 5). The total silver level in the March 31 sample was $131 \mu \mathrm{g} / \mathrm{L}$, which is within the acceptable biocidal range (>100 $\mu \mathrm{g} / \mathrm{L})$. The silver biocide levels returned to typical low levels in the SRV-K samples collected on April 26 and May18 (5-9 $\mu \mathrm{g} / \mathrm{L})$, indicating that heating of the water in the galley was the main source of microbial control at that time. The TOC levels in the SRV-K samples ranged from 0.18-0.51 mg/L. Traces of 1,4-dichlorobenzene (1.4 $\mu \mathrm{g} / \mathrm{L})$, dibutylphthalate $(6 \mu \mathrm{g} / \mathrm{L})$, 2-methylthiobenzothiazole $(4 \mu \mathrm{g} / \mathrm{L})$, formaldehyde $(2-6 \mu \mathrm{g} / \mathrm{L})$, and chloroform $(1.7 \mu \mathrm{g} / \mathrm{L})$ were identified in the SRV-K samples, which were not toxicologically significant levels. The TOC levels in the 4 SRV-K water samples ranged from $0.18-0.51 \mu \mathrm{g} / \mathrm{L}$ and were well below the limit (Figure 6). 


\section{SVO-ZV Potable Water Samples}

All chemical parameters measured for the 3 SVO-ZV water samples met requirements listed in the ISS MORD. Manganese ranged from 27-35 $\mu \mathrm{g} / \mathrm{L}$, well below the specification limit (Figure 7). The total silver levels of 132-187 $\mu \mathrm{g} / \mathrm{L}$ were within the acceptable biocidal range $(>100 \mu \mathrm{g} / \mathrm{L})$, but on the lower end of that range which increases the risk of microbial growth (see Figure 8). The TOC levels in the SVO-ZV water samples ranged from 0.48-3.53 mg/L. The only organics identified in the samples were traces of bromodichloromethane $(2.6 \mu \mathrm{g} / \mathrm{L})$, formaldehyde $(3-4$ $\mu \mathrm{g} / \mathrm{L})$, and chloroform (1.4-47.8 $\mu \mathrm{g} / \mathrm{L})$. Although the chloroform level of $47.8 \mu \mathrm{g} / \mathrm{L}$ in the March 31, 2010 sample is higher than the historical average of $13.5 \mu \mathrm{g} / \mathrm{L}$ in SVO-ZV samples, it falls well below the EPA maximum contaminant limit for trihalomethanes of $80 \mu \mathrm{g} / \mathrm{L}$. Accordingly, none of the organics detected were at levels sufficient to pose a crew health risk.

\section{EXPEDITION 24}

A total of 4 chemical archival potable water samples, including 2 PWD ambient, 1 PWD hot, and 1 WPA RIP were collected during Expedition 24 as detailed in Table 1. The 4 samples collected on July 14, 2010, July 29, 2010, August 25, 2010, and September 15, 2010 were returned on Soyuz 22 and received in the WAFAL on September 26, 2010. All of the samples were collected using U.S. 1-liter Teflon ${ }^{\circledR}$ water sample bags. Due to limited sample volume, turbidity was not analyzed on the WPA RIP sample, and solids were not analyzed on any of the samples.

\section{ISS US SEGMENT:}

\section{PWD Potable Water Samples}

All chemical parameters measured for the 3 potable water samples from the PWD met requirements in SSP 41000. The nickel levels (see Figure 1) ranged from $4-29 \mu \mathrm{g} / \mathrm{L}$. The total iodine (I) was $<0.05 \mathrm{mg} / \mathrm{L}$ and met the requirement of $0.2 \mathrm{mg} / \mathrm{L}$ at points of consumption (Figure 2). The iron levels remained below the detection limit (Figure 3). The TOC levels ranged from 1.11-2.19 mg/L, within the $3 \mathrm{mg} / \mathrm{L}$ limit, but trending up beginning July 14, 2010 (see Figure 4). A comparison of in-flight versus archival TOC results is presented in Figure 9. The archival TOC results confirm the trend seen with the TOCA in-flight analyses, although the TOCA results, on average, are $45 \%$ lower than the archive results (Figure 9).

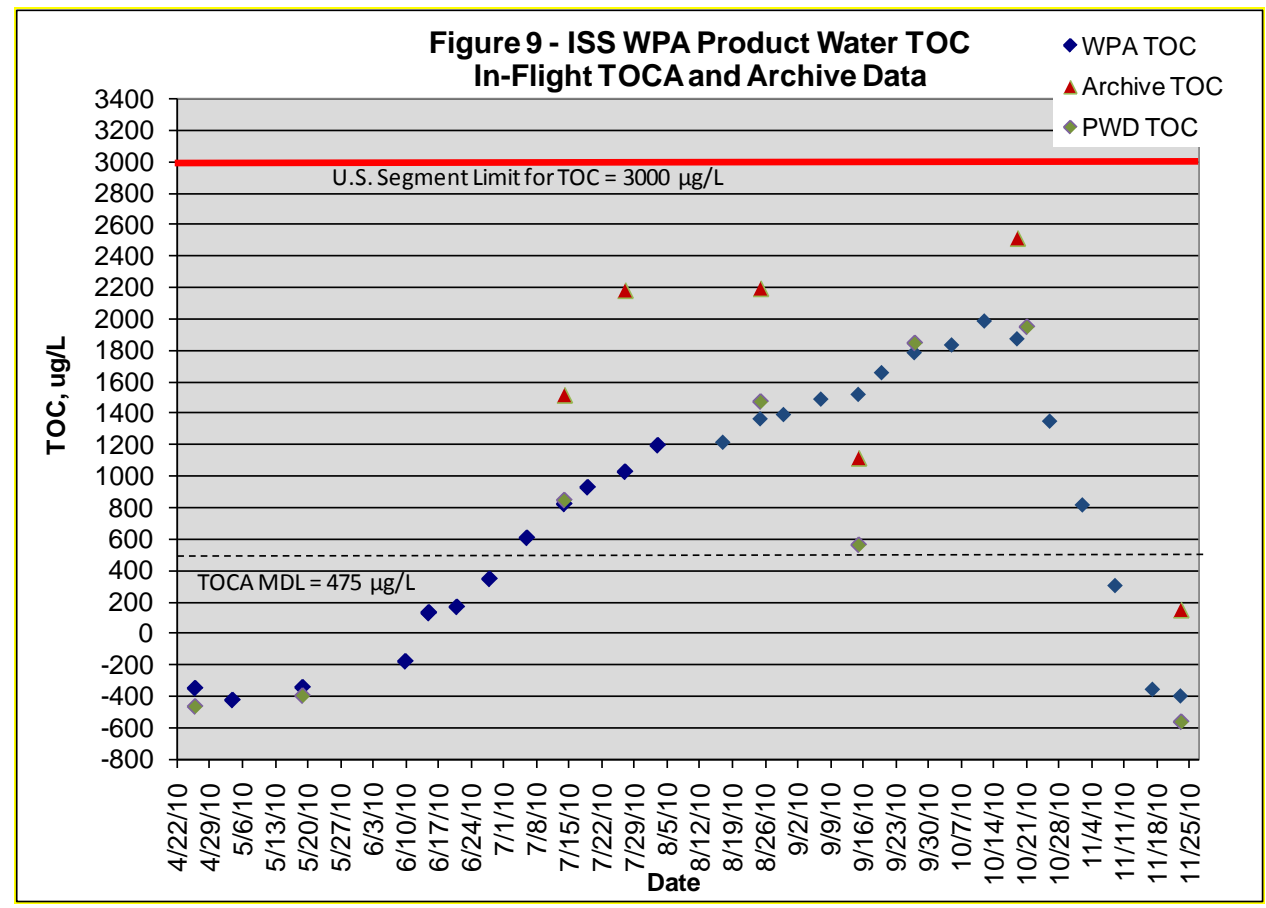

Routine analyses for target organics showed only trace levels of 2-butanone $(<2-4 \mu \mathrm{g} / \mathrm{L})$, methyl sulfone $(<4-36$ $\mu \mathrm{g} / \mathrm{L}$ ) and formaldehyde (5-8 $\mu \mathrm{g} / \mathrm{L})$ in the samples that were toxicologically insignificant. As the target list did not reveal the contaminant responsible for the increase in TOC, efforts to look for unknown compounds were initiated. A method for glycerol was developed but this compound was not detected in any of the potable samples. Further 
work, however, showed an unknown peak in the gas chromatography/mass spectrometry (GC/MS) analysis for glycols. This unknown was identified as dimethylsilanediol (DMSD) by GC/MS analysis. ${ }^{11}$ A method for analysis of DMSD was then developed, which provided estimated DMSD levels from 4.7-8.2 mg/L. The results from the DMSD analysis should be considered estimates only as the synthesized standard used for calibration was not traceable. Using the estimated DMSD levels, the organic carbon accountability for the potable water samples ranged from $98-111 \%$ indicating that DMSD was the primary contributor to the elevated TOC in the WPA product water. An interim SWEG of $25 \mathrm{mg} / \mathrm{L}$ was subsequently established for DMSD. Accordingly, in-flight levels did not pose a crew health risk. ${ }^{12}$

Non-routine silicon analysis by inductively coupled plasma/mass spectrometry (ICP/MS) was also conducted to confirm that the unknown contained silicon and whether there were additional silicon containing contaminants in addition to the DMSD. The results show that the silicon levels ranged from 1.21-2.13 mg/L. These levels are slightly less than values predicted using the estimated DMSD levels, confirming that most of the silicon was accounted for in the DMSD analysis.

More detailed descriptions of the extensive work performed to identify and quantify the DMSD and of the multidisciplinary teamwork used to evaluate associated risk and investigate potential sources and root cause can be found in other recent publications. ${ }^{12,13}$

\section{WPA Processed Water Sample}

The TOC level in the product water sample from the WPA RIP was $2.18 \mathrm{mg} / \mathrm{L}$, which also reflects an upward trend (Figure 4). Methyl sulfone and formaldehyde were identified at toxicologically insignificant levels of $150 \mu \mathrm{g} / \mathrm{L}$ and $8 \mu \mathrm{g} / \mathrm{L}$, respectively. Glycerol was not detected and the silicon level was $1.77 \mathrm{mg} / \mathrm{L}$. The estimated DMSD concentration was $7.3 \mathrm{mg} / \mathrm{L}$, and well below the interim SWEG of $25 \mathrm{mg} / \mathrm{L}$. Organic carbon accountability for the sample improved to $90 \%$, with DMSD as the primary contributor.

\section{EXPEDITION 25}

A total of 3 chemical archival potable water samples, including 2 PWD hot and 1 SRV-K warm, were collected during Expedition 25 as detailed in Table 1. The 3 samples were collected on October 19, 2010 and November 23, 2010, returned on Soyuz 23 and received in the WAFAL on November 29, 2010. All of the samples were collected using U.S. 1-liter Teflon ${ }^{\circledR}$ water sample bags. Due to limited sample volume, total dissolved solids were not analyzed on any of the samples and turbidity was not analyzed on the October 19 PWD sample or the SRV-K sample.

\section{ISS US SEGMENT:}

\section{PWD Potable Water Samples}

All chemical parameters measured for the 2 potable water samples from the PWD met ISS quality requirements listed in SSP 41000. The nickel levels of 17 and $23 \mu \mathrm{g} / \mathrm{L}$ were well within specifications (see Figure 1). The total iodine (I) levels were below the detection limit $(<0.05 \mathrm{mg} / \mathrm{L})$ and met the ISS limit of $0.2 \mathrm{mg} / \mathrm{L}$ at points of consumption (Figure 2). The iron levels remained below the detection limit (Figure 3). The TOC levels of $2.51 \mathrm{mg} / \mathrm{L}$ on October 19 and $0.15 \mathrm{mg} / \mathrm{L}$ on November 23 indicate that the TOC level in the WPA product water continued to rise until mid-October, but never reached the $3 \mathrm{mg} / \mathrm{L}$ limit before falling precipitously (see Figure 4). These results confirm the TOCA in-flight data trend and an offset between TOCA results and archive results (Figure 9). The primary contributor to the elevated TOC again proved to be DMSD. The DMSD level in the October 19 sample of $8.49 \mathrm{mg} / \mathrm{L}$ is the highest seen in product water to date, but still well below the recently established SWEG of 25 $\mathrm{mg} / \mathrm{L}$. As expected, DMSD was not detected $(<2 \mathrm{mg} / \mathrm{L})$ in the November 23 sample collected after the TOC of the WPA product water returned to a nominal low level. Again, these DMSD results should be considered estimates as the synthesized standard used for calibration of the DMSD method was not traceable. Routine analyses for other target organics detected only trace levels of acetone ( $<2$ and $40 \mu \mathrm{g} / \mathrm{L}$ ), 2-butanone ( $<2$ and $24 \mu \mathrm{g} / \mathrm{L}$ ), and methyl sulfone (48 and $50 \mu \mathrm{g} / \mathrm{L}$ ) in the samples. Non-routine silicon analysis by ICP/MS was also performed to confirm the presence of DMSD and determine if there were additional silicon containing contaminants present in the samples. The results indicated silicon levels of 0.94 and $0.22 \mathrm{mg} / \mathrm{L}$ in the October 19 and November 23 samples, respectively. These levels are slightly less than values predicted using the estimated DMSD levels, thereby confirming that most of the silicon in the samples was being accounted for in the DMSD analysis. 
ISS RUSSIAN SEGMENT:

SRV-K Potable Water Sample

All chemical parameters measured for the SRV-K warm water sample were within the ISS MORD requirements. The nickel level of $32 \mu \mathrm{g} / \mathrm{L}$ was well below the specification limit (Figure 5). The silver biocide level of $75 \mu \mathrm{g} / \mathrm{L}$ was below the acceptable biocidal range $(>100 \mu \mathrm{g} / \mathrm{L})$ indicating that heating of the water by the pasteurization unit was the main source of microbial control in the SRV-K galley at the time. As shown in Figure 6, the TOC level was $0.38 \mathrm{mg} / \mathrm{L}$ and well below the specification limit. No specific organics were identified in the sample.

\section{Conclusions}

The chemical analysis results for the archival potable water samples collected and returned during Expeditions 21 through 25 indicate that the ISS potable water supplies complied with ISS quality specifications and were acceptable for crew consumption.

Turbidity levels exceeded the ISS MORD limit of 1.5 NTU in 4 of 8 SVO-ZV samples collected during Expeditions 21 through 25. Although elevated turbidity in itself does not pose a direct crew health risk, the concern is that particulate matter causing the turbidity could shield bacteria from the silver biocide that is added for microbial control. Analytical results for dissolved silver indicate that some of the particulates contributing to elevated turbidity are colloidal silver, which has biocidal properties and may help to mitigate the turbidity concern. The silver biocide levels in 2 of 8 SVO-ZV samples were below the acceptable biocidal range (>100 $\mu \mathrm{g} / \mathrm{L})$, which indicates increased risk of microbial growth. Continued close monitoring of the SVO-ZV silver level is therefore recommended to determine if remedial action is required.

Silver biocide levels in all but one SRV-K sample remained typically low $(<100 \mu \mathrm{g} / \mathrm{L})$, indicating that heating of the water by the pasteurization unit continued to be the primary means of microbial control in the SRV-K galley. The nickel level of $116 \mu \mathrm{g} / \mathrm{L}$ in the March 31, 2010 SRV-K hot sample slightly exceeded the ISS MORD specification of $100 \mu \mathrm{g} / \mathrm{L}$, but was well within the established SWEG of $300 \mu \mathrm{g} / \mathrm{L}$.

From early July to mid-October the TOC level in the WPA product water showed a continuous rise, approaching the $3 \mathrm{mg} / \mathrm{L}$ limit, before falling back to nominal low levels in mid-November. The analytical results for archival PWD and WPA product water samples confirm the TOCA in-flight data trend and an offset between TOCA results and archive results. DMSD was eventually identified as the primary contributor to the elevated TOC. The November 23 PWD sample, collected after the TOC of the WPA product water returned to a nominal low level, did not contain DMSD. It is recommended that WPA troubleshooting support continue as well as support for the multidisciplinary effort to establish root cause and the environmental source(s) of the DMSD.

\section{Appendix}

The chemical analysis results for the archival potable water samples collected from the Russian Segment SRV-K (regenerated water) system during Expeditions 21 through 25 are presented in Appendix 1. Analytical results for the chemical archive samples collected from the Russian Segment SVO-ZV (stored water) system during these 5 expeditions are presented in Appendix 2. Appendix 3 contains the chemical analysis results for U.S. Segment Water Processor Assembly product water samples collected during Expeditions 21-25.

\section{Acknowledgments}

All of the work described in this paper was performed at the JSC Water and Food Analytical Laboratory (WAFAL) under NASA contract NAS9-02078. The authors wish to acknowledge the ISS Expeditions 21-25 crewmembers for collecting and packing the chemical archival samples for return to the ground. Mickie Benoit of the WAFAL is acknowledged for coordinating the retrieval and delivery of the returned ISS samples to WAFAL. The following WAFAL chemists are gratefully acknowledged for performing chemical analyses of the chemical archival water samples: Jim Alverson, Lydia Ding, Mike Kuo, Esther Liu, Jeff Rutz, and Dawn Zapp. Finally, our Russian colleagues, Peter Andreichuk and Elena Zapryagaylo of RSC-Energia, Yuri Sinyak and Sergei Harin of the Institute of Biomedical Problems, and Leonid Bobe of NIICHIMMASH are also acknowledged. 


\section{References}

${ }^{1}$ Straub, J. E., Plumlee, D. K., and Schultz, J. R., “ISS Expeditions 16 thru 20: Chemical Analysis Results for Potable Water”, AIAA-2010-6042, AIAA Proceedings of the 2010 International Conference on Environmental Systems, 2010.

${ }^{2}$ Straub, J. E., Plumlee, D. K., and Schultz, J. R., “Chemical Analysis Results for Potable Water Returned from ISS Expeditions 14 and 15”, SAE International Journal of Aerospace. 1(1): 556-577, 2008.

${ }^{3}$ Straub, J. E., Plumlee, D. K., and Schultz, J. R., "Sampling and Chemical Analysis of Potable Water for ISS Expeditions 12 and 13”, SAE International Journal of Aerospace, 2007.

${ }^{4}$ Straub, J. E., Plumlee, D. K., and Schultz, J. R., "ISS Expeditions 10 \& 11 Potable Water Sampling and Chemical Analysis Results”, SAE International Journal of Aerospace, 2006.

${ }^{5}$ Straub, J. E., Plumlee, D. K., and Schultz, J. R., “Chemical Analysis of ISS Potable Water from Expeditions 8 and 9”, SAE International Journal of Aerospace, 2005.

${ }^{6}$ Straub, J. E., Plumlee, D. K., and Schultz, J. R., “ISS Potable Water Sampling and Chemical Analysis: Expeditions 6 \& 7”, SAE International Journal of Aerospace, 2004.

${ }^{7}$ Plumlee, D. K. and Schultz, J. R., "ISS Potable Water Sampling and Chemical Analysis: Expeditions 4 \& 5", SAE International Journal of Aerospace, 2003.

${ }^{8}$ Plumlee, D. K. and Schultz, J. R., “Chemical Sampling and Analysis of ISS Potable Water: Expeditions 1-3”, SAE International Journal of Aerospace, 2002.

${ }^{9}$ ISS Medical Operations Requirements Document, SSP 50260, Revision C, NASA Johnson Space Center, February 2006, Section 7.2 and Appendix D, Table D-1.

${ }^{10}$ System Specification for the International Space Station, SSP 41000 BP, National Aeronautics and Space Administration, September 28, 2009, Table LXX.

${ }^{11}$ Bentley, N, et al., "Second-Generation International Space Station Total Organic Carbon Analyzer Verification Testing and OnOrbit Performance Results”, AIAA-2010-6043, AIAA Proceedings of the 2011 International Conference on Environmental Systems, 2010.

${ }^{12}$ Rutz, J. A. et al., "Discovery and Identification of Dimethylsilanediol as a Contaminant in ISS Potable Water”, AIAA Technical Paper \#1021758. 41 ${ }^{\text {st }}$ International Conference on Environmental Systems, 2011.

${ }^{13}$ McCoy, J. T. et al., "The Story Behind the Numbers: Lessons Learned from the Integration of Monitoring Resources in Addressing an ISS Water Quality Anomaly”, AIAA Technical Paper \#1020578 . $41^{\text {st }}$ International Conference on Environmental Systems, 2011. 
Appendix 1. ISS SRV-K Potable Water (Regenerated) Summary for Expeditions 21 through 25

\begin{tabular}{|c|c|c|c|c|c|c|c|c|c|c|c|c|c|c|}
\hline Mission & & & & & ISS ULF3/Exp. 21 & ISS 20A & /Exp. 22 & Soyuz 20 & Exp. 22 & Soyuz 21/Exp. 23 & & S ULF4/Exp. 2 & & \begin{tabular}{|l|} 
Soyuz 23/Exp. 25 \\
\end{tabular} \\
\hline $\begin{array}{l}\text { Sample Location } \\
\text { Sample Description } \\
\text { Sample Date } \\
\text { Analysis/Sample ID }\end{array}$ & Units & $\begin{array}{c}\text { Test } \\
\text { Conducted } \\
\text { by }\end{array}$ & $\begin{array}{l}\text { Potable W ater } \\
\text { Maximum } \\
\text { Contaminant } \\
\text { Level } \\
\text { (MCL) }\end{array}$ & $\begin{array}{c}\text { Maximum } \\
\text { Contaminant } \\
\text { Level } \\
\text { Source } \\
\end{array}$ & $\begin{array}{l}\text { SRV-K Hot } \\
\\
\text { Potable Water } \\
11 / 10 / 2009 \\
20091130011 \\
\end{array}$ & \begin{tabular}{|c|} 
SRV-K Warm \\
\\
Potable Water \\
$1 / 6 / 2010$ \\
20100222006 \\
\end{tabular} & \begin{tabular}{|c|} 
SRV-K Hot \\
\\
Potable Water \\
2/3/2010 \\
20100222008 \\
\end{tabular} & $\begin{array}{c}\text { SRV-K Warm } \\
\\
\text { Potable Water } \\
3 / 3 / 2010 \\
20100326002 \\
\end{array}$ & \begin{tabular}{|c|} 
SRV-K Hot \\
\\
Potable Water \\
$3 / 3 / 2010$ \\
20100326003 \\
\end{tabular} & $\begin{array}{c}\text { Potable W ater } \\
3 / 31 / 2010 \\
20100603001\end{array}$ & \begin{tabular}{|c|} 
SRV-K W arm \\
\\
Potable Water \\
$4 / 26 / 2010$ \\
20100527010 \\
\end{tabular} & \begin{tabular}{|c|} 
Potable Water \\
$5 / 18 / 2010$ \\
20100527011
\end{tabular} & \begin{tabular}{|c|} 
SRV-K Hot \\
\\
Potable Water \\
$5 / 18 / 2010$ \\
20100527012 \\
\end{tabular} & \begin{tabular}{c|} 
SRV-K Warm \\
\\
Potable Water \\
$11 / 23 / 2010$ \\
20101129003 \\
\end{tabular} \\
\hline Physical Characteristics & & & & & & & & & & & & & & \\
\hline $\begin{array}{l}\text { Pnysical Cnaracteristics } \\
\mathrm{pH}\end{array}$ & $\mathrm{pH}$ units & U.S. & $5.5-9.0$ & MORD & 7.31 & 7.09 & 7.00 & 7.45 & 7.34 & 6.33 & 6.51 & 6.43 & 6.35 & 5.82 \\
\hline Conductivity & $\mu \mathrm{S} / \mathrm{cm}$ & U.S. & & & 15 & 14 & 189 & 49 & 50 & 187 & 20 & 22 & 21 & 124 \\
\hline Turbidity & \begin{tabular}{|l|l|} 
NTU \\
\end{tabular} & U.S. & $1.5^{*}$ & MORD & 0.4 & 0.2 & 0.4 & 0.5 & 0.2 & 0.5 & 0.2 & 0.3 & 0.4 & $\mathrm{NA}$ \\
\hline \begin{tabular}{|l|l|} 
Total Dissolved Solids \\
\end{tabular} & \begin{tabular}{|l|}
$\mathrm{mg} / \mathrm{L}$ \\
\end{tabular} & U.S. & $100\left(1,000^{\#}\right)$ & MORD & $<5$ & 15 & 112 & $\mathrm{NA}$ & $\mathrm{NA}$ & NA & $<5$ & $\mathrm{NA}$ & $<5$ & $\mathrm{NA}$ \\
\hline Iodine (LCV) & & & & & & & & & & & & & & \\
\hline Total I & \begin{tabular}{|l|}
$\mathrm{mg} / \mathrm{L}$ \\
\end{tabular} & U.S. & 0.05 & MORD & $<0.05$ & $<0.05$ & $<0.05$ & $<0.05$ & $<0.05$ & $<0.05$ & $<0.05$ & $<0.05$ & $<0.05$ & $<0.05$ \\
\hline & & & & & & & & & & & & & & \\
\hline Anions (IC/ISE) & & & & & & & & & & & & & & \\
\hline Bromide & $\mathrm{mg} / \mathrm{L}$ & U.S. & & & $<0.5$ & $<0.5$ & $<0.5$ & $<0.5$ & $<0.5$ & $<0.5$ & $<0.5$ & $<0.5$ & $<0.5$ & $<0.5$ \\
\hline \begin{tabular}{|l|} 
Chloride \\
\end{tabular} & \begin{tabular}{|l|}
$\mathrm{mg} / \mathrm{L}$ \\
\end{tabular} & U.S. & 250 & MORD & $<0.15$ & $<0.15$ & 4.47 & 1.09 & 1.14 & 4.89 & 0.21 & 0.18 & 0.19 & 4.37 \\
\hline \begin{tabular}{|l|} 
Fluoride \\
\end{tabular} & \begin{tabular}{|l|}
$\mathrm{mg} / \mathrm{L}$ \\
\end{tabular} & U.S. & $1.5 / 4$ & MORD/EPA & $<0.1$ & $<0.1$ & $<0.1$ & $<0.1$ & $<0.1$ & $<0.1$ & $<0.1$ & $<0.1$ & $<0.1$ & $<0.1$ \\
\hline \begin{tabular}{|l} 
Nitrate as Nitrogen (NO3-N) \\
\end{tabular} & \begin{tabular}{|l|}
$\mathrm{mg} / \mathrm{L}$ \\
\end{tabular} & U.S. & 10 & MORD/EPA & $<0.11$ & $<0.11$ & 0.16 & $<0.11$ & $<0.11$ & $<0.11$ & $<0.11$ & $<0.11$ & $<0.11$ & 0.38 \\
\hline \begin{tabular}{|l} 
Nitrite as Nitrogen (NO2-N) \\
\end{tabular} & \begin{tabular}{|l|}
$\mathrm{mg} / \mathrm{L}$ \\
\end{tabular} & U.S. & 1 & EPA & $\mathrm{NA}$ & $\mathrm{NA}$ & $\mathrm{NA}$ & $\mathrm{NA}$ & NA & NA & $\mathrm{NA}$ & $\mathrm{NA}$ & NA & $\mathrm{NA}$ \\
\hline Phosphate as P (PO4-P) & \begin{tabular}{|l|l|l}
$\mathrm{mg} / \mathrm{L}$ \\
\end{tabular} & U.S. & & & $<0.24$ & $<0.24$ & $<0.24$ & $<0.24$ & $<0.24$ & $<0.24$ & $<0.24$ & $<0.24$ & $<0.24$ & $<0.02$ \\
\hline \begin{tabular}{|l|l|} 
Sulfate \\
\end{tabular} & \begin{tabular}{|l|}
$\mathrm{mg} / \mathrm{L}$ \\
\end{tabular} & U.S. & 250 & MORD & $<0.75$ & $<0.75$ & 13.5 & 0.94 & 1.02 & 19.2 & $<0.75$ & $<0.75$ & $<0.75$ & 11.6 \\
\hline \begin{tabular}{|l} 
Cations.(IC) \\
\end{tabular} & & & & & & & & & & & & & & \\
\hline Ammonia as Nitrogen (NH3-N) & \begin{tabular}{|l|}
$\mathrm{mg} / \mathrm{L}$ \\
\end{tabular} & U.S. & $2 / 1$ & MORD/SWEG & $<0.002$ & $<0.002$ & $<0.002$ & $<0.002$ & $<0.002$ & 0.018 & $<0.002$ & $<0.002$ & $<0.002$ & $<0.002$ \\
\hline Lithium & \begin{tabular}{|l|}
$\mathrm{mg} / \mathrm{L}$ \\
\end{tabular} & U.S. & & & $<0.002$ & $<0.002$ & $<0.002$ & $<0.002$ & $<0.002$ & $<0.002$ & $<0.002$ & $<0.002$ & $<0.002$ & $<0.002$ \\
\hline Metals (ICP/M S) & & & & & & & & & & & & & & \\
\hline \begin{tabular}{|l} 
Calcium \\
\end{tabular} & \begin{tabular}{|l|}
$\mathrm{mg} / \mathrm{L}$ \\
\end{tabular} & U.S. & 100 & MORD & 2.42 & 2.34 & 28.0 & 9.36 & 9.61 & 21.1 & 3.23 & 3.17 & 3.17 & 18.1 \\
\hline \begin{tabular}{|l} 
Magnesium \\
\end{tabular} & \begin{tabular}{|l|}
$\mathrm{mg} / \mathrm{L}$ \\
\end{tabular} & U.S. & 50 & MORD & 0.03 & 0.04 & 4.21 & 0.16 & 0.16 & 5.34 & 0.17 & 0.21 & 0.21 & $\frac{1.14}{4.14}$ \\
\hline Potassium & \begin{tabular}{|l|}
$\mathrm{mg} / \mathrm{L}$ \\
\end{tabular} & U.S. & & & 0.04 & $<0.01$ & 0.72 & 0.01 & $<0.01$ & 1.71 & 0.02 & 0.04 & 0.03 & 1.03 \\
\hline Sodium & \begin{tabular}{|l|}
$\mathrm{mg} / \mathrm{L}$ \\
\end{tabular} & U.S. & & & 0.01 & 0.02 & 2.12 & 0.01 & 0.01 & 3.88 & 0.13 & 0.15 & 0.15 & 5.12 \\
\hline Aluminum & \begin{tabular}{|l|}
$\mu \mathrm{g} / \mathrm{L}$ \\
\end{tabular} & U.S. & & & $<2$ & $<2$ & 19 & 3 & 4 & 53 & 6 & $<4$ & 4 & 101 \\
\hline \begin{tabular}{|l|} 
Antimony \\
\end{tabular} & \begin{tabular}{|l|}
$\mu \mathrm{g} / \mathrm{L}$ \\
\end{tabular} & U.S. & 6 & EPA & $<2$ & $<2$ & $<2$ & $<2$ & $<2$ & $<2$ & $<2$ & $<4$ & $<2$ & $<4$ \\
\hline \begin{tabular}{|l|} 
Arsenic \\
\end{tabular} & \begin{tabular}{|l|}
$\mu g / L / L$ \\
\end{tabular} & U.S. & 10 & MORD/EPA & $<1$ & $<1$ & $<1$ & $<1$ & $<1$ & $<1$ & $<1$ & $<2$ & $<1$ & $<2$ \\
\hline Barium & \begin{tabular}{|l|}
$\mu \mathrm{g} / \mathrm{L}$ \\
\end{tabular} & U.S. & $1,000 / 10,000$ & MORD/SWEG & $<1$ & $<1$ & 11 & $<1$ & $<1$ & 8 & $<1$ & $<2$ & $<1$ & 12 \\
\hline Beryllium & \begin{tabular}{|l|}
$\mu \mathrm{g} / \mathrm{L}$ \\
\end{tabular} & U.S. & 4 & EPA & $<1$ & $<1$ & $<1$ & $<1$ & $<1$ & $<1$ & $<1$ & $<2$ & $<1$ & $<2$ \\
\hline \begin{tabular}{|l|} 
Cadmium \\
\end{tabular} & \begin{tabular}{|l|}
$\mu \mathrm{g} / \mathrm{L}$ \\
\end{tabular} & U.S. & $5 / 22$ & MORD/SWEG & $<1$ & $<1$ & $<1$ & $<1$ & $<1$ & 1 & $<1$ & $<2$ & $<1$ & $<2$ \\
\hline \begin{tabular}{|l} 
Chromium \\
\end{tabular} & \begin{tabular}{|l|}
$\mu g / L / L$ \\
\end{tabular} & U.S. & 100 & MORD/EPA & $<5$ & $<5$ & $<5$ & $<5$ & $<5$ & $<5$ & $<5$ & $<10$ & $<5$ & $<10$ \\
\hline Cobalt & \begin{tabular}{|l|}
$\mu \mathrm{g} / \mathrm{L}$ \\
\end{tabular} & U.S. & & & $<1$ & $<1$ & $<1$ & $<1$ & $<1$ & $<1$ & $<1$ & $<2$ & $<1$ & $<2$ \\
\hline Copper & \begin{tabular}{|l|}
$\mu \mathrm{g} / \mathrm{L}$ \\
\end{tabular} & U.S. & $1,000 / 1,300$ & MORD/EPA & 11 & 4 & 7 & 2 & 9 & 9 & 7 & 15 & 13 & 5 \\
\hline Iron & \begin{tabular}{|l|}
$\mu \mathrm{g} / \mathrm{L}$ \\
\end{tabular} & U.S. & 300 & MORD & 6 & 6 & 39 & 17 & 15 & 36 & $<5$ & $<10$ & $<5$ & 19 \\
\hline Lead & \begin{tabular}{|l|}
$\mu \mathrm{g} / \mathrm{L}$ \\
\end{tabular} & U.S. & $50 / 15$ & MORD/EPA & $<1$ & $<1$ & $<1$ & $<1$ & $<1$ & 1 & $<1$ & $<2$ & $<1$ & $<2$ \\
\hline Manganese & \begin{tabular}{|l|}
$\mu g / L / L$ \\
\end{tabular} & U.S. & $50 / 300$ & MORD/SWEG & $<1$ & $<1$ & 13 & $<1$ & $<1$ & 28 & 1 & $<2$ & 1 & $\frac{2}{6}$ \\
\hline Mercury & \begin{tabular}{|l|}
$\mu \mathrm{g} / \mathrm{L}$ \\
\end{tabular} & U.S. & 2 & MORD/EPA & $<0.5$ & $<0.5$ & $<0.5$ & $<0.5$ & $<0.5$ & $<0.5$ & $<0.5$ & $<0.5$ & $<0.5$ & $<1$ \\
\hline \begin{tabular}{|l|} 
Molybdenum \\
\end{tabular} & \begin{tabular}{|l|}
$\mu \mathrm{g} / \mathrm{L}$ \\
\end{tabular} & U.S. & 40 & EPA HA & $<1$ & $<1$ & $<1$ & $<1$ & $<1$ & $<1$ & $<1$ & $<2$ & $<1$ & $<2$ \\
\hline Nickel & \begin{tabular}{|l|}
$\mu \mathrm{g} / \mathrm{L}$ \\
\end{tabular} & U.S. & $100 / 300$ & MORD/SWEG & 34 & 48 & 26 & 43 & 39 & 116 & 36 & 68 & 46 & 32 \\
\hline Selenium & \begin{tabular}{|l|}
$\mu g / L / L$ \\
\end{tabular} & U.S. & $10 / 50$ & MORD/EPA & $<1$ & $<1$ & $<1$ & $<1$ & $<1$ & $<1$ & $<1$ & $<2$ & $<1$ & $<2$ \\
\hline Silver & \begin{tabular}{|l|}
$\mu \mathrm{g} / \mathrm{L}$ \\
\end{tabular} & U.S. & $500 / 400$ & MORD/SWEG & 14 & 7 & 108 & 7 & 3 & 131 & 5 & 7 & 9 & 75 \\
\hline Silver, Dissolved & \begin{tabular}{|l|}
$\mu \mathrm{g} / \mathrm{L}$ \\
\end{tabular} & U.S. & & & 5 & 2 & 90 & $<4$ & $<4$ & 58 & $<2$ & $<4$ & 3 & 57 \\
\hline Zinc & $\mu \mathrm{g} / \mathrm{L}$ & U.S. & $5,000 / 2,000$ & MORD/SWEG & 59 & 69 & 27 & 27 & 21 & 54 & 34 & 129 & 42 & 18 \\
\hline & & & & & & & & & & & & & & \\
\hline Silicon (ICP/MS) & & & & & & & & & & & & & & \\
\hline Silicon (ICP/MS) & \begin{tabular}{|l|}
$\mu \mathrm{g} / \mathrm{L}$ \\
\end{tabular} & U.S. & & & $\mathrm{NA}$ & $\mathrm{NA}$ & NA & $\mathrm{NA}$ & $\mathrm{NA}$ & $\mathrm{NA}$ & $\mathrm{NA}$ & NA & 73 & 793 \\
\hline & & & & & & & & & & & & & & \\
\hline Total Organic Carbon (S & & & & & & & & & & & & & & \\
\hline Total Inorganic Carbon & \begin{tabular}{|l|}
$\mathrm{mg} / \mathrm{L}$ \\
\end{tabular} & U.S. & & & 2.79 & 2.64 & 18.1 & 6.33 & 6.44 & 17.2 & 3.62 & 3.54 & 3.58 & 14.2 \\
\hline
\end{tabular}

NA=Not analyzed; MI=Matrix interference
${ }^{*}$ MORD limit $1.5 \mathrm{mg} / \mathrm{L}$ (Russian method)

*limit does not include contribution from formate

SWEG - 1000 days (5-2006) 
Appendix 1. ISS SRV-K Potable Water (Regenerated) Summary for Expeditions 21 through 25

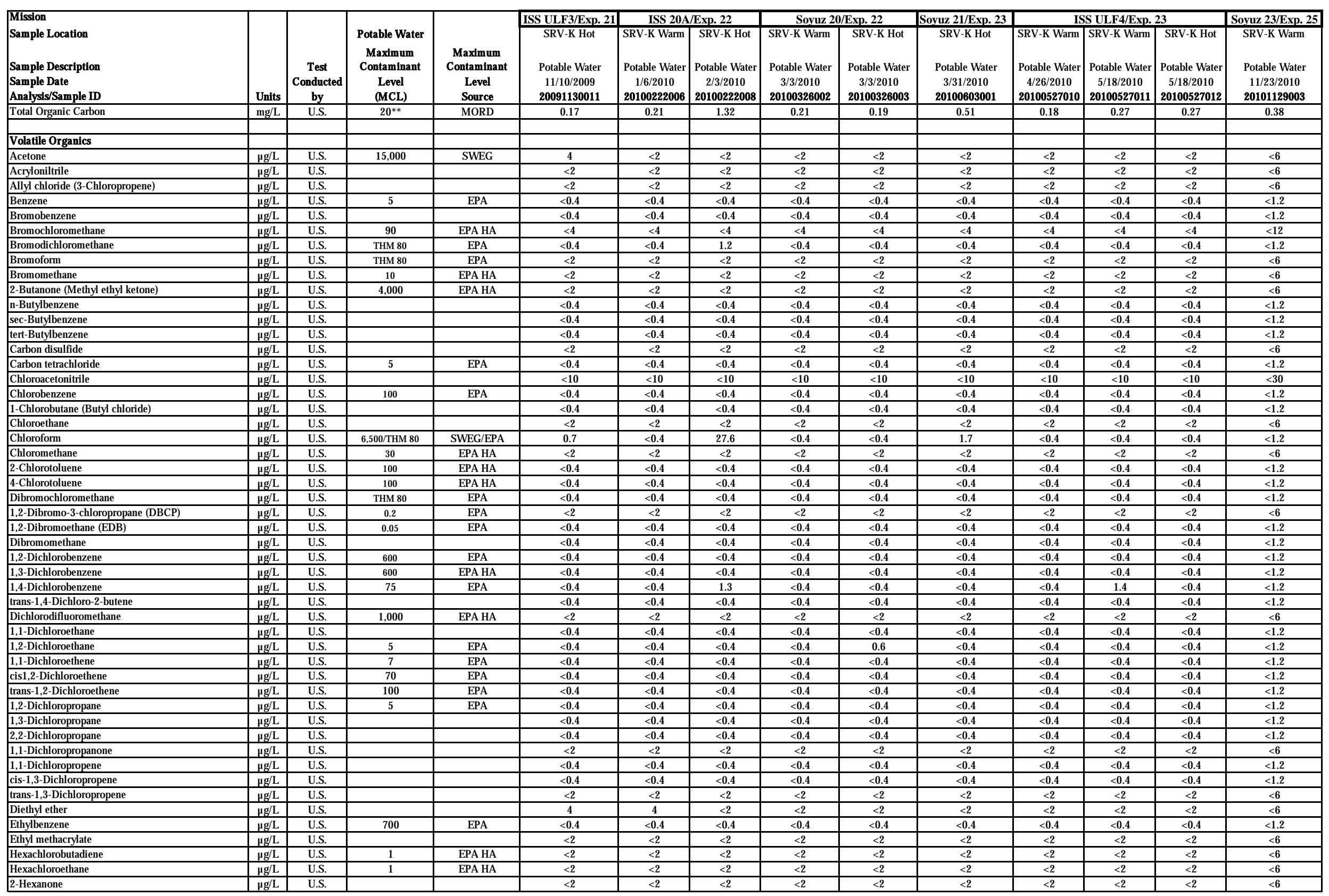

NA=Not analyzed; MI=Matrix interference
${ }^{*}$ MORD limit $1.5 \mathrm{mg} / \mathrm{L}$ (Russian method)

* *limit does not include contribution from formate

SWEG - 1000 days (5-2006) 
Appendix 1. ISS SRV-K Potable Water (Regenerated) Summary for Expeditions 21 through 25

\begin{tabular}{|c|c|c|c|c|c|c|c|c|c|c|c|c|c|c|}
\hline Mission & & & & & ISS ULF3/Exp. 21 & ISS 20A & $\sqrt{\text { Exp. } 22}$ & Soyuz 20 & Exp. 22 & Soyuz 21/Exp. 23 & & S ULF4/Exp. 2 & & \begin{tabular}{|l|} 
Soyuz 23/Exp. 25 \\
\end{tabular} \\
\hline Sample Location & & & Potable W ater & & SRV-K Hot & SRV-K Warm & SRV-K Hot & SRV-K Warm & SRV-K Hot & SRV-K Hot & \begin{tabular}{|l|} 
SRV-K Warm \\
\end{tabular} & SRV-K Warm & \begin{tabular}{|l|} 
SRV-K Hot \\
\end{tabular} & \\
\hline $\begin{array}{l}\text { Sample Description } \\
\text { Sample Date } \\
\text { Analysis/Sample ID }\end{array}$ & Units & $\begin{array}{c}\text { Test } \\
\text { Conducted } \\
\text { by }\end{array}$ & $\begin{array}{c}\text { Maximum } \\
\text { Contaminant } \\
\text { Level } \\
\text { (MCL) } \\
\end{array}$ & $\begin{array}{c}\text { Maximum } \\
\text { Contaminant } \\
\text { Level } \\
\text { Source } \\
\end{array}$ & $\begin{array}{c}\text { Potable Water } \\
11 / 10 / 2009 \\
20091130011 \\
\end{array}$ & \begin{tabular}{|c|} 
Potable W ater \\
$1 / 6 / 2010$ \\
20100222006 \\
\end{tabular} & \begin{tabular}{|c|} 
Potable Water \\
$2 / 3 / 2010$ \\
20100222008 \\
\end{tabular} & $\begin{array}{c}\text { Potable Water } \\
3 / 3 / 2010 \\
20100326002 \\
\end{array}$ & $\begin{array}{c}\text { Potable Water } \\
3 / 3 / 2010 \\
20100326003\end{array}$ & $\begin{array}{c}\text { Potable Water } \\
3 / 31 / 2010 \\
20100603001\end{array}$ & \begin{tabular}{|c|} 
Potable W ater \\
$4 / 26 / 2010$ \\
20100527010 \\
\end{tabular} & \begin{tabular}{|c|} 
Potable W ater \\
$5 / 18 / 2010$ \\
20100527011 \\
\end{tabular} & \begin{tabular}{|c|} 
Potable Water \\
$5 / 18 / 2010$ \\
20100527012 \\
\end{tabular} & $\begin{array}{c}\text { Potable Water } \\
11 / 23 / 2010 \\
20101129003\end{array}$ \\
\hline \begin{tabular}{|l|} 
Iodomethane \\
\end{tabular} & $\mu \mathrm{g} / \mathrm{L}$ & \begin{tabular}{l|l} 
U.S. & \\
\end{tabular} & & & $<2$ & $<2$ & $<2$ & $<2$ & $<2$ & $<2$ & $<2$ & $<2$ & $<2$ & $<6$ \\
\hline Isopropylbenzene (Cumene) & $\mu \mathrm{g} / \mathrm{L}$ & U.S. & 4,000 & EPA DWEL & $<0.4$ & $<0.4$ & $<0.4$ & $<0.4$ & $<0.4$ & $<0.4$ & $<0.4$ & $<0.4$ & $<0.4$ & $<1.2$ \\
\hline 4-Isopropyltoluene (Cymene) & $\mu \mathrm{g} / \mathrm{L}$ & U.S. & & & $<0.4$ & $<0.4$ & $<0.4$ & $<0.4$ & $<0.4$ & $<0.4$ & $<0.4$ & $<0.4$ & $<0.4$ & $<1.2$ \\
\hline Methacrylonitrile & $\mu \mathrm{g} / \mathrm{L}$ & U.S. & & & $<2$ & $<2$ & $<2$ & $<2$ & $<2$ & $<2$ & $<2$ & $<2$ & $<2$ & $<6$ \\
\hline Methyl acrylate & $\mu \mathrm{g} / \mathrm{L}$ & U.S. & & & $<2$ & $<2$ & $<2$ & $<2$ & $<2$ & $<2$ & $<2$ & $<2$ & $<2$ & $<6$ \\
\hline Methyl-t-butylether (MTBE) & $\mu \mathrm{g} / \mathrm{L}$ & U.S. & & & $<2$ & $<2$ & $<2$ & $<2$ & $<2$ & $<2$ & $<2$ & $<2$ & $<2$ & $<6$ \\
\hline Methylene chloride (Dichloromethane) & $\mu \mathrm{g} / \mathrm{L}$ & U.S. & $15,000 / 5$ & SWEG/EPA & $<0.4$ & $<0.4$ & $<0.4$ & $<0.4$ & $<0.4$ & $<0.4$ & $<0.4$ & $<0.4$ & $<0.4$ & $<1.2$ \\
\hline \begin{tabular}{|l|} 
Methyl methacrylate \\
\end{tabular} & $\mu \mathrm{g} / \mathrm{L}$ & U.S. & & & $<2$ & $<2$ & $<2$ & $<2$ & $<2$ & $<2$ & $<2$ & $<2$ & $<2$ & $<6$ \\
\hline 4-Methyl-2-pentanone & $\mu \mathrm{g} / \mathrm{L}$ & U.S. & & & $<0.4$ & $<0.4$ & $<0.4$ & $<0.4$ & $<0.4$ & $<0.4$ & $<0.4$ & $<0.4$ & $<0.4$ & $<1.2$ \\
\hline \begin{tabular}{|l} 
Naphthalene \\
\end{tabular} & $\mu \mathrm{g} / \mathrm{L}$ & U.S. & 100 & EPA HA & $<0.4$ & $<0.4$ & $<0.4$ & $<0.4$ & $<0.4$ & $<0.4$ & $<0.4$ & $<0.4$ & $<0.4$ & $<1.2$ \\
\hline Nitrobenzene & $\mu \mathrm{g} / \mathrm{L}$ & U.S. & & & $<2$ & $<2$ & $<2$ & $<2$ & $<2$ & $<2$ & $<2$ & $<2$ & $<2$ & $<6$ \\
\hline 2-Nitropropane & $\mu \mathrm{g} / \mathrm{L}$ & U.S. & & & $<2$ & $<2$ & $<2$ & $<2$ & $<2$ & $<2$ & $<2$ & $<2$ & $<2$ & $<6$ \\
\hline Pentachloroethane & $\mu \mathrm{g} / \mathrm{L}$ & U.S. & & & $<2$ & $<2$ & $<2$ & $<2$ & $<2$ & $<2$ & $<2$ & $<2$ & $<2$ & $<6$ \\
\hline \begin{tabular}{|l|} 
Propionitrile (Ethyl cyanide) \\
\end{tabular} & $\mu \mathrm{g} / \mathrm{L}$ & U.S. & & & $<10$ & $<10$ & $<10$ & $<10$ & $<10$ & $<10$ & $<10$ & $<10$ & $<10$ & $<30$ \\
\hline n-Propylbenzene & $\mu \mathrm{g} / \mathrm{L}$ & U.S. & & & $<0.4$ & $<0.4$ & $<0.4$ & $<0.4$ & $<0.4$ & $<0.4$ & $<0.4$ & $<0.4$ & $<0.4$ & $<1.2$ \\
\hline \begin{tabular}{|l|l|} 
Styrene \\
\end{tabular} & $\mu \mathrm{g} / \mathrm{L}$ & U.S. & 100 & EPA & $<0.4$ & $<0.4$ & $<0.4$ & $<0.4$ & $<0.4$ & $<0.4$ & $<0.4$ & $<0.4$ & $<0.4$ & $<1.2$ \\
\hline 1,1,1,2-Tetrachloroethane & $\mu \mathrm{g} / \mathrm{L}$ & U.S. & 70 & EPA HA & $<0.4$ & $<0.4$ & $<0.4$ & $<0.4$ & $<0.4$ & $<0.4$ & $<0.4$ & $<0.4$ & $<0.4$ & $<1.2$ \\
\hline 1,1,2,2-Tetrachloroethane & $\mu \mathrm{g} / \mathrm{L}$ & U.S. & 0.3 & EPA HA & $<0.4$ & $<0.4$ & $<0.4$ & $<0.4$ & $<0.4$ & $<0.4$ & $<0.4$ & $<0.4$ & $<0.4$ & $<1.2$ \\
\hline Tetrachloroethene & $\mu \mathrm{g} / \mathrm{L}$ & U.S. & 5 & EPA & $<0.4$ & $<0.4$ & $<0.4$ & $<0.4$ & $<0.4$ & $<0.4$ & $<0.4$ & $<0.4$ & $<0.4$ & $<1.2$ \\
\hline Tetrahydrofuran & $\mu \mathrm{g} / \mathrm{L}$ & U.S. & & & $<2$ & $<2$ & $<2$ & $<2$ & $<2$ & $<2$ & $<2$ & $<2$ & $<2$ & $<6$ \\
\hline \begin{tabular}{|l|} 
Toluene \\
\end{tabular} & $\mu \mathrm{g} / \mathrm{L}$ & U.S. & 1,000 & EPA & $<0.4$ & $<0.4$ & $<0.4$ & $<0.4$ & $<0.4$ & $<0.4$ & $<0.4$ & $<0.4$ & $<0.4$ & $<1.2$ \\
\hline 1,2,3-Trichlorobenzene & $\mu \mathrm{g} / \mathrm{L}$ & U.S. & & & $<0.4$ & $<0.4$ & $<0.4$ & $<0.4$ & $<0.4$ & $<0.4$ & $<0.4$ & $<0.4$ & $<0.4$ & $<1.2$ \\
\hline 1,2,4-Trichlorobenzene & $\mu \mathrm{g} / \mathrm{L}$ & U.S. & 70 & EPA & $<0.4$ & $<0.4$ & $<0.4$ & $<0.4$ & $<0.4$ & $<0.4$ & $<0.4$ & $<0.4$ & $<0.4$ & $<1.2$ \\
\hline 1,1,1-Trichloroethane & $\mu \mathrm{g} / \mathrm{L}$ & U.S. & 200 & EPA & $<0.4$ & $<0.4$ & $<0.4$ & $<0.4$ & $<0.4$ & $<0.4$ & $<0.4$ & $<0.4$ & $<0.4$ & $<1.2$ \\
\hline \begin{tabular}{|l}
$1,1,2$-Trichloroethane \\
\end{tabular} & $\mu \mathrm{g} / \mathrm{L}$ & U.S. & 5 & EPA & $<0.4$ & $<0.4$ & $<0.4$ & $<0.4$ & $<0.4$ & $<0.4$ & $<0.4$ & $<0.4$ & $<0.4$ & $<1.2$ \\
\hline Trichloroethene & $\mu \mathrm{g} / \mathrm{L}$ & U.S. & 5 & EPA & $<0.4$ & $<0.4$ & $<0.4$ & $<0.4$ & $<0.4$ & $<0.4$ & $<0.4$ & $<0.4$ & $<0.4$ & $<1.2$ \\
\hline Trichlorofluoromethane & $\mu \mathrm{g} / \mathrm{L}$ & U.S. & 2,000 & EPA HA & $<0.4$ & $<0.4$ & $<0.4$ & $<0.4$ & $<0.4$ & $<0.4$ & $<0.4$ & $<0.4$ & $<0.4$ & $<1.2$ \\
\hline 1,2,3-Trichloropropane & $\mu \mathrm{g} / \mathrm{L}$ & U.S. & 40 & EPA HA & $<0.4$ & $<0.4$ & $<0.4$ & $<0.4$ & $<0.4$ & $<0.4$ & $<0.4$ & $<0.4$ & $<0.4$ & $<1.2$ \\
\hline 1,2,4-Trimethylbenzene & $\mu \mathrm{g} / \mathrm{L}$ & U.S. & & & $<0.4$ & $<0.4$ & $<0.4$ & $<0.4$ & $<0.4$ & $<0.4$ & $<0.4$ & $<0.4$ & $<0.4$ & $<1.2$ \\
\hline 1,3,5-Trimethylbenzene & $\mu \mathrm{g} / \mathrm{L}$ & U.S. & & & $<0.4$ & $<0.4$ & $<0.4$ & $<0.4$ & $<0.4$ & $<0.4$ & $<0.4$ & $<0.4$ & $<0.4$ & $<1.2$ \\
\hline \begin{tabular}{|l|} 
Vinyl Acetate \\
\end{tabular} & $\mu \mathrm{g} / \mathrm{L}$ & U.S. & & & $<2$ & $<2$ & $<2$ & $<2$ & $<2$ & $<2$ & $<2$ & $<2$ & $<2$ & $<6$ \\
\hline Vinyl Chloride & $\mu \mathrm{g} / \mathrm{L}$ & U.S. & 2 & EPA & $<2$ & $<2$ & $<2$ & $<2$ & $<2$ & $<2$ & $<2$ & $<2$ & $<2$ & $<6$ \\
\hline \begin{tabular}{|l|} 
m\&p-Xylene \\
\end{tabular} & $\mu \mathrm{g} / \mathrm{L}$ & U.S. & Total Xylenes 10,000 & EPA & $<0.4$ & $<0.4$ & $<0.4$ & $<0.4$ & $<0.4$ & $<0.4$ & $<0.4$ & $<0.4$ & $<0.4$ & $<1.2$ \\
\hline o-Xylene & $\mu \mathrm{g} / \mathrm{L}$ & U.S. & Total Xylenes 10,000 & EPA & $<0.4$ & 1.5 & $<0.4$ & $<0.4$ & $<0.4$ & $<0.4$ & $<0.4$ & $<0.4$ & $<0.4$ & $<1.2$ \\
\hline & & & & & & & & & & & & & & \\
\hline Volatile Organics - Non-T argets (T ent & ied Com & pounds $(>1=8$ & $80 \%$ match quality & & & & & & & & & & & \\
\hline \begin{tabular}{|l|} 
Acetaldehyde \\
\end{tabular} & $\mu \mathrm{g} / \mathrm{L}$ & U.S. & & & 3 & not found & not found & not found & not found & not found & not found & not found & not found & not found \\
\hline Butyraldehyde (Butanal) & $\mu \mathrm{g} / \mathrm{L}$ & U.S. & & & not found & not found & not found & not found & not found & not found & not found & not found & not found & not found \\
\hline \begin{tabular}{|l|}
1,8 -Cineole \\
\end{tabular} & $\mu \mathrm{g} / \mathrm{L}$ & U.S. & & & not found & not found & not found & not found & not found & not found & not found & not found & not found & not found \\
\hline \begin{tabular}{|l|} 
Cyclohexanone \\
\end{tabular} & $\mu \mathrm{g} / \mathrm{L}$ & U.S. & & & not found & not found & not found & not found & not found & not found & not found & not found & not found & not found \\
\hline \begin{tabular}{|l|l|} 
Difluorodimethylsilane \\
\end{tabular} & $\mu \mathrm{g} / \mathrm{L}$ & & & & not found & not found & not found & not found & not found & not found & not found & not found & not found & not found \\
\hline Dimethoxymethane (Formal) & $\mu \mathrm{g} / \mathrm{L}$ & U.S. & & & not found & not found & not found & not found & not found & not found & not found & not found & not found & not found \\
\hline 2,5-Dimethylfuran & $\mu \mathrm{g} / \mathrm{L}$ & U.S. & & & not found & not found & not found & not found & not found & not found & not found & not found & not found & not found \\
\hline 2,6-Dimethyl-1,7-octadiene & $\mu \mathrm{g} / \mathrm{L}$ & U.S. & & & not found & not found & not found & not found & not found & not found & not found & not found & not found & not found \\
\hline \begin{tabular}{|l|l} 
Dimethyl sulfide (Thiobismethane) \\
\end{tabular} & $\mu \mathrm{g} / \mathrm{L}$ & & & & not found & not found & not found & not found & not found & not found & not found & not found & not found & not found \\
\hline p-Dioxane & $\mu \mathrm{g} / \mathrm{L}$ & U.S. & & & not found & not found & not found & not found & not found & not found & not found & not found & not found & not found \\
\hline 1,3-Dioxolane (Ethylene glycol formal) & $\mu \mathrm{g} / \mathrm{L}$ & U.S. & & & not found & not found & not found & not found & not found & not found & not found & not found & not found & not found \\
\hline Ethyl acetate & $\mu \mathrm{g} / \mathrm{L}$ & U.S. & & & not found & not found & not found & not found & not found & not found & not found & not found & not found & not found \\
\hline \begin{tabular}{|l|} 
Fluorotrimethylsilane \\
\end{tabular} & $\mu \mathrm{g} / \mathrm{L}$ & U.S. & & & not found & not found & not found & not found & not found & not found & not found & not found & not found & not found \\
\hline 2-Heptanone & $\mu \mathrm{g} / \mathrm{L}$ & U.S. & & & not found & not found & not found & not found & not found & not found & not found & not found & not found & not found \\
\hline 3-Hexanone & $\mu \mathrm{g} / \mathrm{L}$ & U.S. & & & not found & not found & not found & not found & not found & not found & not found & not found & not found & not found \\
\hline 3-Hydroxy-3-methylbutyric acid & $\mu \mathrm{g} / \mathrm{L}$ & U.S. & & & not found & not found & not found & not found & not found & not found & not found & not found & not found & not found \\
\hline
\end{tabular}


Appendix 1. ISS SRV-K Potable Water (Regenerated) Summary for Expeditions 21 through 25

\begin{tabular}{|c|c|c|c|c|c|c|c|c|c|c|c|c|c|c|}
\hline Mission & & & & & ISS ULF3/Exp. 21 & ISS 20A & $\sqrt{\text { Exp. } 22}$ & Soyuz 20 & Exp. 22 & Soyuz 21/Exp. 23 & & SS ULF4/Exp. 2 & & \begin{tabular}{|l|} 
Soyuz 23/Exp. 25 \\
\end{tabular} \\
\hline Sample Location & & & Potable Water & & SRV-K Hot & SRV-K Warm & SRV-K Hot & SRV-K Warm & SRV-K Hot & SRV-K Hot & SRV-K Warm & SRV-K Warm & SRV-K Hot & SRV-K Warm \\
\hline $\begin{array}{l}\text { Sample Description } \\
\text { Sample Date } \\
\text { Analysis/Sample ID }\end{array}$ & Units & $\begin{array}{c}\text { Test } \\
\text { Conducted } \\
\text { by }\end{array}$ & $\begin{array}{c}\text { Maximum } \\
\text { Contaminant } \\
\text { Level } \\
\text { (MCL) } \\
\end{array}$ & $\begin{array}{c}\text { Maximum } \\
\text { Contaminant } \\
\text { Level } \\
\text { Source } \\
\end{array}$ & $\begin{array}{l}\text { Potable Water } \\
11 / 10 / 2009 \\
20091130011\end{array}$ & \begin{tabular}{|c|} 
Potable Water \\
$1 / 6 / 2010$ \\
20100222006 \\
\end{tabular} & \begin{tabular}{|c|} 
Potable Water \\
$2 / 3 / 2010$ \\
20100222008 \\
\end{tabular} & $\begin{array}{c}\text { Potable Water } \\
3 / 3 / 2010 \\
20100326002 \\
\end{array}$ & $\begin{array}{c}\text { Potable W ater } \\
3 / 3 / 2010 \\
20100326003 \\
\end{array}$ & $\begin{array}{c}\text { Potable Water } \\
3 / 31 / 2010 \\
20100603001\end{array}$ & \begin{tabular}{|c|} 
Potable W ater \\
$4 / 26 / 2010$ \\
20100527010 \\
\end{tabular} & \begin{tabular}{|c|} 
Potable Water \\
$5 / 18 / 2010$ \\
20100527011 \\
\end{tabular} & \begin{tabular}{|c|} 
Potable Water \\
$5 / 18 / 2010$ \\
20100527012 \\
\end{tabular} & $\begin{array}{c}\text { Potable Water } \\
11 / 23 / 2010 \\
20101129003 \\
\end{array}$ \\
\hline Isobutyronitrile & $\mu \mathrm{g} / \mathrm{L}$ & U.S. & & & not found & not found & not found & not found & not found & not found & not found & not found & not found & not found \\
\hline Isooctanol & $\mu \mathrm{g} / \mathrm{L}$ & U.S. & & & not found & not found & not found & not found & not found & not found & not found & not found & not found & not found \\
\hline L-Menthol & $\mu \mathrm{g} / \mathrm{L}$ & U.S. & & & not found & not found & not found & not found & not found & not found & not found & not found & not found & not found \\
\hline Menthone & $\mu \mathrm{g} / \mathrm{L}$ & U.S. & & & not found & not found & not found & not found & not found & not found & not found & not found & not found & not found \\
\hline \begin{tabular}{|l} 
Methyl acetate \\
\end{tabular} & $\mu \mathrm{g} / \mathrm{L}$ & U.S. & & & not found & not found & not found & not found & not found & not found & not found & not found & not found & not found \\
\hline cis-1-Methyl-4-(1-methylethenyl)-cyclohexane & $\mu \mathrm{g} / \mathrm{L}$ & U.S. & & & not found & not found & not found & not found & not found & not found & not found & not found & not found & not found \\
\hline 3-Methyl-2-pentanone & $\mu \mathrm{g} / \mathrm{L}$ & U.S. & & & not found & not found & not found & not found & not found & not found & not found & not found & not found & not found \\
\hline 2-Methyl-1-propene & $\mu \mathrm{g} / \mathrm{L}$ & U.S. & & & not found & not found & not found & not found & not found & not found & not found & not found & not found & not found \\
\hline \begin{tabular}{|l|} 
alpha-Methyl styrene \\
\end{tabular} & $\mu \mathrm{g} / \mathrm{L}$ & U.S. & & & not found & not found & not found & not found & not found & not found & not found & not found & not found & not found \\
\hline 2-Nonanone & $\mu \mathrm{g} / \mathrm{L}$ & U.S. & & & not found & not found & not found & not found & not found & not found & not found & not found & not found & not found \\
\hline 2 -0ctanone & $\mu \mathrm{g} / \mathrm{L}$ & U.S. & & & not found & not found & not found & not found & not found & not found & not found & not found & not found & not found \\
\hline 2-Pentanone & $\mu \mathrm{g} / \mathrm{L}$ & U.S. & & & not found & not found & not found & not found & not found & not found & not found & not found & not found & not found \\
\hline 1,1,2-Trichloro-1,2,2-trifluoroethane (Freon 113) & $\mu \mathrm{g} / \mathrm{L}$ & U.S. & & & not found & not found & not found & not found & not found & not found & not found & not found & not found & not found \\
\hline Trimethylsilanol & $\mu \mathrm{g} / \mathrm{L}$ & U.S. & & & not found & not found & not found & not found & not found & not found & not found & not found & not found & not found \\
\hline & & & & & & & & & & & & & & \\
\hline \begin{tabular}{|l} 
Extractable Organics \\
Actonhenone
\end{tabular} & $810 / \mathrm{I}$ & & & & & & & & & & & & & \\
\hline \begin{tabular}{|l|} 
Acetophenone \\
Benzaldehyde \\
\end{tabular} & $\begin{array}{l}\mu \mathrm{g} / \mathrm{L} \\
\mu \mathrm{g} / \mathrm{L}\end{array}$ & $\begin{array}{l}\text { U.S. } \\
\text { U.S. }\end{array}$ & & & $\begin{array}{l}<8 \\
<4\end{array}$ & $\begin{array}{l}<8 \\
<4\end{array}$ & $\begin{array}{l}<8 \\
<4\end{array}$ & $\begin{array}{l}<16 \\
<8\end{array}$ & $<16$ & $\frac{<16}{<8}$ & $\begin{array}{l}<8 \\
<4\end{array}$ & $\frac{<16}{<8}$ & $<8$ & $\begin{array}{c}<16 \\
<8\end{array}$ \\
\hline \begin{tabular}{|l|} 
Benzoic acid \\
\end{tabular} & $\mu \mathrm{g} / \mathrm{L}$ & U.S. & & & $<12$ & $<12$ & $<12$ & $<24$ & $<24$ & $<24$ & $<12$ & $<24$ & $<12$ & $<48$ \\
\hline \begin{tabular}{|l|} 
Benzothiazole \\
\end{tabular} & $\mu \mathrm{g} / \mathrm{L}$ & U.S. & & & $<4$ & $<4$ & $<4$ & $<8$ & $<8$ & $<8$ & $<4$ & $<8$ & $<4$ & $<8$ \\
\hline Benzyl alcohol & $\mu \mathrm{g} / \mathrm{L}$ & U.S. & & & $<4$ & $<4$ & $<4$ & $<8$ & $<8$ & $<8$ & $<4$ & $<8$ & $<4$ & $<8$ \\
\hline \begin{tabular}{|l} 
Benzyl butyl phthlate \\
\end{tabular} & $\mu \mathrm{g} / \mathrm{L}$ & U.S. & 7,000 & EPA DWEL & $<4$ & $<4$ & $<4$ & $<8$ & $<8$ & $<8$ & $<4$ & $<8$ & $<4$ & $<8$ \\
\hline 2-Butoxyethanol & $\mu \mathrm{g} / \mathrm{L}$ & U.S. & & & $<8$ & $<8$ & $<8$ & $<16$ & $<16$ & $<16$ & $<8$ & $<16$ & $<8$ & $<16$ \\
\hline 2-(2-Butoxyethoxy)ethanol & $\mu \mathrm{g} / \mathrm{L}$ & U.S. & & & $<8$ & $<8$ & $<8$ & $<16$ & $<16$ & $<16$ & $<8$ & $<16$ & $<8$ & $<16$ \\
\hline 2-(2-Butoxyethoxy)ethyl acetate & $\mu \mathrm{g} / \mathrm{L}$ & U.S. & & & $<4$ & $<4$ & $<4$ & $<8$ & $<8$ & $<8$ & $<4$ & $<8$ & $<4$ & $<8$ \\
\hline $\mathrm{n}$-Butylpalmitate & $\mu \mathrm{g} / \mathrm{L}$ & U.S. & & & $<8$ & $<8$ & $<8$ & $<16$ & $<16$ & $<16$ & $<8$ & $<16$ & $<8$ & $<16$ \\
\hline Butylated hydroxyanisole (BHA) & $\mu \mathrm{g} / \mathrm{L}$ & U.S. & & & $<4$ & $<4$ & $<4$ & $<8$ & $<8$ & $<8$ & $<4$ & $<8$ & $<4$ & $<8$ \\
\hline N-Butylbenzenesulfonamide & $\mu \mathrm{g} / \mathrm{L}$ & U.S. & & & $<4$ & $<4$ & $<4$ & $<8$ & $<8$ & $<8$ & $<4$ & $<8$ & $<4$ & $<8$ \\
\hline 3-tert-Butylphenol & $\mu \mathrm{g} / \mathrm{L}$ & U.S. & & & $<12$ & $<12$ & $<12$ & $<24$ & $<24$ & $<24$ & $<12$ & $<24$ & $<12$ & $<24$ \\
\hline \begin{tabular}{|l|} 
Caffeine \\
\end{tabular} & $\mu \mathrm{g} / \mathrm{L}$ & U.S. & & & $<4$ & $<4$ & $<4$ & $<8$ & $<8$ & $<8$ & $<4$ & $<8$ & $<4$ & $<8$ \\
\hline tris-2-Chloroethyl phosphate & $\mu \mathrm{g} / \mathrm{L}$ & U.S. & & & $<4$ & $<4$ & $<4$ & $<8$ & $<8$ & $<8$ & $<4$ & $<8$ & $<4$ & $<8$ \\
\hline \begin{tabular}{|l|} 
Cholesterol \\
\end{tabular} & $\mu \mathrm{g} / \mathrm{L}$ & U.S. & & & $<32$ & $<32$ & $<32$ & $<64$ & $<64$ & $<64$ & $<32$ & $<64$ & $<32$ & $<64$ \\
\hline o-Cresol (2-Methylphenol) & $\mu \mathrm{g} / \mathrm{L}$ & U.S. & & & $<4$ & $<4$ & $<4$ & $<8$ & $<8$ & $<8$ & $<4$ & $<8$ & $<4$ & $<8$ \\
\hline Cyclododecane & $\mu \mathrm{g} / \mathrm{L}$ & U.S. & & & $<4$ & $<4$ & $<4$ & $<8$ & $<8$ & $<8$ & $<4$ & $<8$ & $<4$ & $<8$ \\
\hline \begin{tabular}{|l} 
Decamethylcyclopentasiloxane \\
\end{tabular} & $\mu \mathrm{g} / \mathrm{L}$ & U.S. & & & $<4$ & $<4$ & $<4$ & $<8$ & $<8$ & $<8$ & $<4$ & $<8$ & $<4$ & $<8$ \\
\hline Decanoic acid & $\mu \mathrm{g} / \mathrm{L}$ & U.S. & & & $<8$ & $<8$ & $<8$ & $<16$ & $<16$ & $<16$ & $<8$ & $<16$ & $<8$ & $<24$ \\
\hline 2,6-Di-t-butyl-1,4-benzoquinone & $\mu \mathrm{g} / \mathrm{L}$ & U.S. & & & $<4$ & $<4$ & $<4$ & $<8$ & $<8$ & $<8$ & $<4$ & $<8$ & $<4$ & $<8$ \\
\hline 2,4-Di-t-butylphenol & $\mu \mathrm{g} / \mathrm{L}$ & U.S. & & & $<4$ & $<4$ & $<4$ & $<8$ & $<8$ & $<8$ & $<4$ & $<8$ & $<4$ & $<8$ \\
\hline 1,4 Diacetylbenzene & $\mu \mathrm{g} / \mathrm{L}$ & U.S. & & & $<4$ & $<4$ & $<4$ & $<8$ & $<8$ & $<8$ & $<4$ & $<8$ & $<4$ & $<8$ \\
\hline N,N-Dibutylformamide & $\mu \mathrm{g} / \mathrm{L}$ & U.S. & & & $<4$ & $<4$ & $<4$ & $<8$ & $<8$ & $<8$ & $<4$ & $<8$ & $<4$ & $<8$ \\
\hline \begin{tabular}{|l|l} 
Dibutyl phthalate \\
\end{tabular} & $\mu \mathrm{g} / \mathrm{L}$ & U.S. & $40,000 / 4,000$ & SWEG/EPA DWEL & 8 & $<4$ & 5 & $<8$ & $<8$ & $<8$ & $<4$ & $<8$ & 6 & $<8$ \\
\hline \begin{tabular}{|l} 
Dibutylamine \\
\end{tabular} & $\mu \mathrm{g} / \mathrm{L}$ & U.S. & Dialkylamines 300 & SWEG & $<4$ & $<4$ & $<4$ & $<8$ & $<8$ & $<8$ & $<4$ & $<8$ & $<4$ & $<8$ \\
\hline N,N-Diethyl-m-toluamide & $\mu \mathrm{g} / \mathrm{L}$ & U.S. & & & $<4$ & $<4$ & $<4$ & $<8$ & $<8$ & $<8$ & $<4$ & $<8$ & $<4$ & $<8$ \\
\hline Diethylphthalate & $\mu \mathrm{g} / \mathrm{L}$ & U.S. & 30,000 & EPA DWEL & $<4$ & $<4$ & $<4$ & $<8$ & $<8$ & $<8$ & $<4$ & $<8$ & $<4$ & $<8$ \\
\hline \begin{tabular}{|l|} 
Diethylene glycol monoethyl ether \\
\end{tabular} & $\mu \mathrm{g} / \mathrm{L}$ & U.S. & & & $<4$ & $<4$ & $<4$ & $<8$ & $<8$ & $<8$ & $<4$ & $<8$ & $<4$ & $<8$ \\
\hline N,N-Diethylformamide & $\mu \mathrm{g} / \mathrm{L}$ & U.S. & & & $<12$ & $<12$ & $<12$ & $<24$ & $<24$ & $<24$ & $<12$ & $<24$ & $<12$ & $<24$ \\
\hline Diiodomethane (Methyl iodide) & $\mu \mathrm{g} / \mathrm{L}$ & U.S. & & & $<4$ & $<4$ & $<4$ & $<8$ & $<8$ & $<8$ & $<4$ & $<8$ & $<4$ & $<8$ \\
\hline \begin{tabular}{|l|} 
Diisopropyl adipate \\
\end{tabular} & $\mu \mathrm{g} / \mathrm{L}$ & U.S. & & & $<4$ & $<4$ & $<4$ & $<8$ & $<8$ & $<8$ & $<4$ & $<8$ & $<4$ & $<8$ \\
\hline \begin{tabular}{|l|} 
Dimethyl phthalate \\
\end{tabular} & $\mu \mathrm{g} / \mathrm{L}$ & U.S. & & & $<4$ & $<4$ & $<4$ & $<8$ & $<8$ & $<8$ & $<4$ & $<8$ & $<4$ & $<8$ \\
\hline N,N-Dimethyl acetamide & $\mu \mathrm{g} / \mathrm{L}$ & U.S. & & & $<4$ & $<4$ & $<4$ & $<8$ & $<8$ & $<8$ & $<4$ & $<8$ & $<4$ & $<8$ \\
\hline \begin{tabular}{|l|}
$\mathrm{N}, \mathrm{N}$-Dimethylbenzylamine \\
\end{tabular} & $\mu \mathrm{g} / \mathrm{L}$ & U.S. & \begin{tabular}{|l|l|} 
Dialkylamines 300 \\
\end{tabular} & SWEG & $<4$ & $<4$ & $<4$ & $<8$ & $<8$ & $<8$ & $<4$ & $<8$ & $<4$ & $<8$ \\
\hline N,N-Dimethylformamide & $\mu \mathrm{g} / \mathrm{L}$ & U.S. & & & $<8$ & $<8$ & $<8$ & $<16$ & $<16$ & $<16$ & $<8$ & $<16$ & $<8$ & $<16$ \\
\hline Dipropylene glycol methyl ether & $\mu \mathrm{g} / \mathrm{L}$ & U.S. & & & $<4$ & $<4$ & $<4$ & $<8$ & $<8$ & $<8$ & $<4$ & $<8$ & $<4$ & $<8$ \\
\hline
\end{tabular}

NA=Not analyzed; MI=Matrix interference
${ }^{*}$ MORD limit $1.5 \mathrm{mg} / \mathrm{L}$ (Russian method)

**limit does not include contribution from formate

\#IDS allowable limit atter min
SWEG - 1000 days (5-2006) 
Appendix 1. ISS SRV-K Potable Water (Regenerated) Summary for Expeditions 21 through 25

\begin{tabular}{|c|c|c|c|c|c|c|c|c|c|c|c|c|c|c|}
\hline \multirow{2}{*}{\begin{tabular}{|l} 
Mission \\
Sample Location
\end{tabular}} & \multirow[b]{3}{*}{ Units } & \multirow[b]{3}{*}{$\begin{array}{c}\text { Test } \\
\text { Conducted } \\
\text { by }\end{array}$} & \multirow{3}{*}{$\begin{array}{l}\text { Potable Water } \\
\text { Maximum } \\
\text { Contaminant } \\
\text { Level } \\
\text { (MCL) }\end{array}$} & \multirow[b]{3}{*}{$\begin{array}{c}\text { Maximum } \\
\text { Contaminant } \\
\text { Level } \\
\text { Source }\end{array}$} & \multirow{3}{*}{\begin{tabular}{|c|} 
ISS ULF3/Exp. 21 \\
SRV-K Hot \\
\\
Potable Water \\
11/10/2009 \\
20091130011 \\
\end{tabular}} & \multicolumn{2}{|c|}{ ISS 20A/Exp. 22} & \multicolumn{2}{|c|}{ Soyuz 20/Exp. 22} & \multirow{3}{*}{\begin{tabular}{|c|} 
Soyuz 21/Exp. 23 \\
SRV-K Hot \\
\\
Potable Water \\
3/31/2010 \\
$\mathbf{2 0 1 0 0 6 0 3 0 0 1}$ \\
\end{tabular}} & \multicolumn{3}{|c|}{ ISS ULF4/Exp. 23} & \multirow{3}{*}{\begin{tabular}{|c|} 
Soyuz 23/Exp. 25 \\
SRV-K Warm \\
\\
Potable Water \\
11/23/2010 \\
$\mathbf{2 0 1 0 1 1 2 9 0 0 3}$ \\
\end{tabular}} \\
\hline & & & & & & \begin{tabular}{|l|} 
SRV-K W arm \\
\end{tabular} & \begin{tabular}{|l|} 
SRV-K Hot \\
\end{tabular} & SRV-K Warm & $\begin{array}{ll}\text { SRV-K Hot } \\
\end{array}$ & & \begin{tabular}{l|l|} 
SRV-K Warm \\
\end{tabular} & SRV-K Warm & SRV-K Hot & \\
\hline $\begin{array}{l}\text { Sample Description } \\
\text { Sample Date } \\
\text { Analysis/Sample ID }\end{array}$ & & & & & & \begin{tabular}{|c|} 
\\
Potable Water \\
$1 / 6 / 2010$ \\
20100222006
\end{tabular} & \begin{tabular}{|c|} 
Potable Water \\
$2 / 3 / 2010$ \\
20100222008
\end{tabular} & $\begin{array}{c}\text { Potable Water } \\
3 / 3 / 2010 \\
20100326002\end{array}$ & $\begin{array}{c}\text { Potable Water } \\
3 / 3 / 2010 \\
20100326003\end{array}$ & & $\begin{array}{c}\text { Potable Water } \\
4 / 26 / 2010 \\
20100527010\end{array}$ & $\begin{array}{c}\text { Potable Water } \\
5 / 18 / 2010 \\
20100527011\end{array}$ & \begin{tabular}{|c|} 
Potable Water \\
$5 / 18 / 2010$ \\
20100527012
\end{tabular} & \\
\hline Dodecamethylcyclohexasiloxane & $\mu \mathrm{g} / \mathrm{L}$ & \begin{tabular}{|l|} 
U.S. \\
\end{tabular} & & & $<4$ & \begin{tabular}{|c|}
$<4$ \\
\end{tabular} & $<4$ & $<8$ & $<8$ & $<8$ & $<4$ & $<8$ & $<4$ & $<8$ \\
\hline 2-Ethoxyethanol & $\mu \mathrm{g} / \mathrm{L}$ & U.S. & & & $<4$ & $<4$ & $<4$ & $<8$ & $<8$ & $<8$ & $<4$ & $<8$ & $<4$ & $<16$ \\
\hline 2-Ethyl-1-hexanol & $\mu \mathrm{g} / \mathrm{L}$ & U.S. & & & $<4$ & $<4$ & $<4$ & $<8$ & $<8$ & $<8$ & $<4$ & $<8$ & $<4$ & $<8$ \\
\hline 2-Ethylhexanoic acid & $\mu \mathrm{g} / \mathrm{L}$ & U.S. & & & $<4$ & $<4$ & $<4$ & $<8$ & $<8$ & $<8$ & $<4$ & $<8$ & $<4$ & $<16$ \\
\hline bis-2-Ethylhexyl adipate & $\mu \mathrm{g} / \mathrm{L}$ & U.S. & 400 & EPA & $<4$ & $<4$ & $<4$ & $<8$ & $<8$ & $<8$ & $<4$ & $<8$ & $<4$ & $<8$ \\
\hline bis-2-Ethylhexyl phthalate (Dioctyl phthlate) & $\mu \mathrm{g} / \mathrm{L}$ & U.S. & $20,000 / 6$ & SWEG/EPA & $<4$ & $<4$ & $<4$ & $<8$ & $<8$ & $<8$ & $<4$ & $<8$ & $<4$ & $<8$ \\
\hline \begin{tabular}{|l|} 
4-Ethylmorpholine \\
\end{tabular} & $\mu \mathrm{g} / \mathrm{L}$ & U.S. & & & $<4$ & $<4$ & $<4$ & $<8$ & $<8$ & $<8$ & $<4$ & $<8$ & $<4$ & $<8$ \\
\hline 1-Formylpiperidine & $\mu \mathrm{g} / \mathrm{L}$ & U.S. & & & $<4$ & $<4$ & $<4$ & $<8$ & $<8$ & $<8$ & $<4$ & $<8$ & $<4$ & $<8$ \\
\hline Heptanoic acid & $\frac{\mu g / L}{\mu g / L}$ & U.S. & & & $<4$ & $<4$ & $<4$ & $<8$ & $<8$ & $<8$ & $<4$ & $<8$ & $<4$ & $<24$ \\
\hline 2-Heptanone & $\mu \mathrm{g} / \mathrm{L}$ & U.S. & & & $<4$ & $<4$ & $<4$ & $<8$ & $<8$ & $<8$ & $<4$ & $<8$ & $<4$ & $<8$ \\
\hline gamma-Hexalactone & $\mu \mathrm{g} / \mathrm{L}$ & U.S. & & & $<4$ & $<4$ & $<4$ & $<8$ & $<8$ & $<8$ & $<4$ & $<8$ & $<4$ & $<8$ \\
\hline \begin{tabular}{|l} 
Hexanoic acid \\
\end{tabular} & $\mu \mathrm{g} / \mathrm{L}$ & U.S. & & & $<8$ & $<8$ & $<8$ & $<16$ & $<16$ & $<16$ & $<8$ & $<16$ & $<8$ & $<24$ \\
\hline 2-Hexanol & $\mu \mathrm{g} / \mathrm{L}$ & U.S. & & & $<4$ & $<4$ & $<4$ & $<8$ & $<8$ & $<8$ & $<4$ & $<8$ & $<4$ & $<8$ \\
\hline 2-Hydroxybenzothiazole & $\mu \mathrm{g} / \mathrm{L}$ & U.S. & & & $<4$ & $<4$ & $<4$ & $<8$ & $<8$ & $<8$ & $<4$ & $<8$ & $<4$ & $<8$ \\
\hline Ibuprofen & $\mu \mathrm{g} / \mathrm{L}$ & U.S. & & & $<4$ & $<4$ & $<4$ & $<8$ & $<8$ & $<8$ & $<4$ & $<8$ & $<4$ & $<24$ \\
\hline Iodoform & $\mu \mathrm{g} / \mathrm{L}$ & U.S. & & & $<4$ & $<4$ & $<4$ & $<8$ & $<8$ & $<8$ & $<4$ & $<8$ & $<4$ & $<8$ \\
\hline Isophorone & $\mu \mathrm{g} / \mathrm{L}$ & U.S. & 100 & EPA HA & $<4$ & $<4$ & $<4$ & $<8$ & $<8$ & $<8$ & $<4$ & $<8$ & $<4$ & $<8$ \\
\hline 4-Isopropylphenol & $\mu \mathrm{g} / \mathrm{L}$ & U.S. & & & $<4$ & $<4$ & $<4$ & $<8$ & $<8$ & $<8$ & $<4$ & $<8$ & $<4$ & $<8$ \\
\hline Lauramide & $\mu \mathrm{g} / \mathrm{L}$ & U.S. & & & $<4$ & $<4$ & $<4$ & $<8$ & $<8$ & $<8$ & $<4$ & $<8$ & $<4$ & $<8$ \\
\hline Lauric acid (Dodecanoic acid) & $\mu \mathrm{g} / \mathrm{L}$ & U.S. & & & $<120$ & $<120$ & $<120$ & $<240$ & $<240$ & $<240$ & $<120$ & $<240$ & $<120$ & $<240$ \\
\hline p-Menth-1-en-8-ol (alpha-Terpineol) & $\mu \mathrm{g} / \mathrm{L}$ & U.S. & & & $<4$ & $<4$ & $<4$ & $<8$ & $<8$ & $<8$ & $<4$ & $<8$ & $<4$ & $<8$ \\
\hline 2-Mercaptobenzothiazole & $\frac{\mu g / L}{\mu g}$ & U.S. & 30,000 & SWEG & $<40$ & $<40$ & $<40$ & $<80$ & $<80$ & $<80$ & $<40$ & $<80$ & $<40$ & $<80$ \\
\hline 2-Methyl-2,4-pentanediol & $\mu \mathrm{g} / \mathrm{L}$ & U.S. & & & $<4$ & $<4$ & $<4$ & $<8$ & $<8$ & $<8$ & $<4$ & $<8$ & $<4$ & $<8$ \\
\hline 1-Methyl-2-pyrolidinone & $\mu \mathrm{g} / \mathrm{L}$ & U.S. & & & $<4$ & $<4$ & $<4$ & $<8$ & $<8$ & $<8$ & $<4$ & $<8$ & $<4$ & $<8$ \\
\hline \begin{tabular}{|l|} 
Methyl-4-hydroxybenzoate \\
\end{tabular} & $\mu \mathrm{g} / \mathrm{L}$ & U.S. & & & $<4$ & $<4$ & $<4$ & $<8$ & $<8$ & $<8$ & $<4$ & $<8$ & $<4$ & $<8$ \\
\hline \begin{tabular}{|l|} 
Methyl sulfone \\
\end{tabular} & $\mu \mathrm{g} / \mathrm{L}$ & U.S. & & & $<4$ & $<4$ & $<4$ & $<8$ & $<8$ & $<8$ & $<4$ & $<8$ & $<4$ & $<8$ \\
\hline 2-Methyl butyric acid & $\mu \mathrm{g} / \mathrm{L}$ & U.S. & & & $<12$ & $<12$ & $<12$ & $<24$ & $<24$ & $<24$ & $<12$ & $<24$ & $<12$ & $<24$ \\
\hline 2-Methylthiobenzothiazole & $\mu \mathrm{g} / \mathrm{L}$ & U.S. & & & $<4$ & $<4$ & $<4$ & $<8$ & $<8$ & $<8$ & $<4$ & $<8$ & 4 & $<8$ \\
\hline Monomethyl phthalate & $\mu \mathrm{g} / \mathrm{L}$ & U.S. & & & $<4$ & $<4$ & $<4$ & $<8$ & $<8$ & $<8$ & $<4$ & $<8$ & $<4$ & $<8$ \\
\hline Myristic acid & $\mu \mathrm{g} / \mathrm{L}$ & U.S. & & & $<24$ & $<24$ & $<24$ & $<48$ & $<48$ & $<48$ & $<24$ & $<48$ & $<24$ & $<64$ \\
\hline (+)-Neomenthol & $\frac{\mu g / L}{\mu g / L}$ & U.S. & & & $<4$ & $<4$ & $<4$ & $<8$ & $<8$ & $<8$ & $<4$ & $<8$ & $\frac{21}{<4}$ & $<8$ \\
\hline Nicotine & $\mu \mathrm{g} / \mathrm{L}$ & U.S. & & & $<4$ & $<4$ & $<4$ & $<8$ & $<8$ & $<8$ & $<4$ & $<8$ & $<4$ & $<8$ \\
\hline Nonadecane & $\mu \mathrm{g} / \mathrm{L}$ & U.S. & & & $<4$ & $<4$ & $<4$ & $<8$ & $<8$ & $<8$ & $<4$ & $<8$ & $<4$ & $<8$ \\
\hline Nonanoic acid & $\mu \mathrm{g} / \mathrm{L}$ & U.S. & & & $<12$ & $<12$ & $<12$ & $<24$ & $<24$ & $<24$ & $<12$ & $<24$ & $<12$ & $<24$ \\
\hline 1-Octadecanol & $\frac{\mu g / L}{\mu g / L}$ & U.S. & & & $<12$ & $<12$ & $<12$ & $<24$ & $<24$ & $<24$ & $<12$ & $<24$ & $<12$ & $<24$ \\
\hline \begin{tabular}{|l} 
Octamethylcyclotetrasiloxane \\
\end{tabular} & $\frac{\mu g / L}{\mu g}$ & U.S. & & & $<4$ & $<4$ & $<4$ & $<8$ & $<8$ & $<8$ & $<4$ & $<8$ & $<4$ & $<8$ \\
\hline Octanoic acid & $\mu \mathrm{g} / \mathrm{L}$ & U.S. & & & $<8$ & $<8$ & $<8$ & $<16$ & $<16$ & $<16$ & $<8$ & $<16$ & $<8$ & $<48$ \\
\hline 4-tert-0ctylphenol & $\mu \mathrm{g} / \mathrm{L}$ & U.S. & & & $<4$ & $<4$ & $<4$ & $<8$ & $<8$ & $<8$ & $<4$ & $<8$ & $<4$ & $<8$ \\
\hline Oleic acid & $\mu \mathrm{g} / \mathrm{L}$ & U.S. & & & $<40$ & $<40$ & $<40$ & $<80$ & $<80$ & $<80$ & $<40$ & $<80$ & $<40$ & $<80$ \\
\hline \begin{tabular}{|l|l|} 
Oxindole \\
\end{tabular} & $\frac{\mu g / L}{\mu g / L}$ & U.S. & & & $<4$ & $<4$ & $<4$ & $<8$ & $<8$ & $<8$ & $<4$ & $<8$ & $<4$ & $<8$ \\
\hline Palmitic acid & $\mu \mathrm{g} / \mathrm{L}$ & U.S. & & & $<120$ & $<120$ & $<120$ & $<240$ & $<240$ & $<240$ & $<120$ & $<240$ & $<120$ & $<240$ \\
\hline Palmitoleic acid & $\mu \mathrm{g} / \mathrm{L}$ & U.S. & & & $\mathrm{NA}$ & NA & $\mathrm{NA}$ & $\mathrm{NA}$ & $\mathrm{NA}$ & $\mathrm{NA}$ & $\mathrm{NA}$ & NA & NA & NA \\
\hline \begin{tabular}{|l|} 
Pentacosane \\
\end{tabular} & $\mu \mathrm{g} / \mathrm{L}$ & U.S. & & & $<4$ & $<4$ & $<4$ & $<8$ & $<8$ & $<8$ & $<4$ & $<8$ & $<4$ & $<8$ \\
\hline sec-Phenethyl alcohol & $\frac{\mu g / L}{\mu g / L}$ & U.S. & & & $<4$ & $<4$ & $<4$ & $<8$ & $<8$ & $<8$ & $<4$ & $<8$ & $<4$ & $<8$ \\
\hline Phenol & $\mu \mathrm{g} / \mathrm{L}$ & U.S. & $1,000 / 4,000$ & MORD/SWEG & $<4$ & $<4$ & $<4$ & $<8$ & $<8$ & $<8$ & $<4$ & $<8$ & $<4$ & $<8$ \\
\hline 2-Phenoxyethanol & $\mu \mathrm{g} / \mathrm{L}$ & U.S. & & & $<4$ & $<4$ & $<4$ & $<8$ & $<8$ & $<8$ & $<4$ & $<8$ & $<4$ & $<8$ \\
\hline \begin{tabular}{|l|} 
N-Phenyl-2-naphthylamine \\
\end{tabular} & $\mu \mathrm{g} / \mathrm{L}$ & U.S. & 260,000 & SWEG & 4 & $<4$ & $<4$ & $<8$ & $<8$ & $<8$ & $<4$ & $<8$ & $<4$ & $<8$ \\
\hline 2-Phenyl-2-propanol & $\mu \mathrm{g} / \mathrm{L}$ & U.S. & & & $<4$ & $<4$ & $<4$ & $<8$ & $<8$ & $<8$ & $<4$ & $<8$ & $<4$ & $<8$ \\
\hline 2-Phenylacetic acid & $\frac{\mu g / L}{\mu g}$ & U.S. & & & $<16$ & $<16$ & $<16$ & $<32$ & $<32$ & $<32$ & $<16$ & $<32$ & $<16$ & $<32$ \\
\hline Phenethyl alcohol & $\mu \mathrm{g} / \mathrm{L}$ & U.S. & & & $<4$ & $<4$ & $<4$ & $<8$ & $<8$ & $<8$ & $<4$ & $<8$ & $<4$ & $<8$ \\
\hline 2-Phenylphenol & $\mu \mathrm{g} / \mathrm{L}$ & U.S. & & & $<4$ & $<4$ & $<4$ & $<8$ & $<8$ & $<8$ & $<4$ & $<8$ & $<4$ & $<8$ \\
\hline \begin{tabular}{|l|l|l} 
Salicyclic Acid \\
\end{tabular} & $\mu \mathrm{g} / \mathrm{L}$ & U.S. & & & $<32$ & $<32$ & $<32$ & $<64$ & $<64$ & $<64$ & $<32$ & $<64$ & $<32$ & $<64$ \\
\hline \begin{tabular}{|l|} 
trans-Squalene \\
\end{tabular} & $\frac{\mu g / L}{\mu g / L}$ & U.S. & & & $<8$ & $<8$ & $<8$ & $<16$ & $<16$ & $<8$ & $<8$ & $<16$ & $<8$ & $<16$ \\
\hline
\end{tabular}

NA=Not analyzed; MI=Matrix interference
${ }^{*}$ MORD limit $1.5 \mathrm{mg} / \mathrm{L}$ (Russian method)

*limit does not include contribution from formate

SWEG - 1000 days (5-2006) 
Appendix 1. ISS SRV-K Potable Water (Regenerated) Summary for Expeditions 21 through 25

\begin{tabular}{|c|c|c|c|c|c|c|c|c|c|c|c|c|c|c|}
\hline Mission & & & & & ISS ULF3/Exp. 21 & ISS 20A & /Exp. 22 & Soyuz 20 & Exp. 22 & \begin{tabular}{|l|} 
Soyuz 21/Exp. 23 \\
\end{tabular} & & SS ULF4/Exp. 2 & & \begin{tabular}{|l|} 
Soyuz 23/Exp. 25 \\
\end{tabular} \\
\hline Sample Location & & & Potable W ater & & SRV-K Hot & \begin{tabular}{|l|} 
SRV-K W arm \\
\end{tabular} & SRV-K Hot & SRV-K Warm & SRV-K Hot & SRV-K Hot & \begin{tabular}{|l|} 
SRV-K Warm \\
\end{tabular} & \begin{tabular}{|l|} 
SRV-K Warm \\
\end{tabular} & \begin{tabular}{|l|} 
SRV-K Hot \\
\end{tabular} & \\
\hline $\begin{array}{l}\text { Sample Description } \\
\text { Sample Date } \\
\text { Analysis/Sample ID }\end{array}$ & Units & $\begin{array}{c}\text { Test } \\
\text { Conducted } \\
\text { by }\end{array}$ & $\begin{array}{c}\text { Maximum } \\
\text { Contaminant } \\
\text { Level } \\
\text { (MCL) }\end{array}$ & $\begin{array}{c}\text { Maximum } \\
\text { Contaminant } \\
\text { L evel } \\
\text { Source } \\
\end{array}$ & $\begin{array}{l}\text { Potable Water } \\
11 / 10 / 2009 \\
20091130011\end{array}$ & \begin{tabular}{|c|} 
Potable W ater \\
$1 / 6 / 2010$ \\
20100222006 \\
\end{tabular} & \begin{tabular}{|c|} 
Potable Water \\
$2 / 3 / 2010$ \\
20100222008 \\
\end{tabular} & $\begin{array}{c}\text { Potable Water } \\
3 / 3 / 2010 \\
20100326002\end{array}$ & $\begin{array}{c}\text { Potable Water } \\
3 / 3 / 2010 \\
20100326003\end{array}$ & $\begin{array}{c}\text { Potable Water } \\
3 / 31 / 2010 \\
20100603001\end{array}$ & \begin{tabular}{|c|} 
Potable W ater \\
$4 / 26 / 2010$ \\
20100527010 \\
\end{tabular} & \begin{tabular}{|c|} 
Potable Water \\
$5 / 18 / 2010$ \\
20100527011 \\
\end{tabular} & \begin{tabular}{|c|} 
Potable Water \\
$5 / 18 / 2010$ \\
20100527012 \\
\end{tabular} & $\begin{array}{l}\text { Potable Water } \\
11 / 23 / 2010 \\
20101129003\end{array}$ \\
\hline \begin{tabular}{|l|} 
Stearic acid \\
\end{tabular} & $\mu \mathrm{g} / \mathrm{L}$ & U.S. & & & $\mathrm{NA}$ & $\mathrm{NA}$ & $\mathrm{NA}$ & $\mathrm{NA}$ & $\mathrm{NA}$ & NA & $\mathrm{NA}$ & $\mathrm{NA}$ & $\mathrm{NA}$ & $\mathrm{NA}$ \\
\hline 1-Tetradecanol & $\mu \mathrm{g} / \mathrm{L}$ & U.S. & & & $<4$ & $<4$ & $<4$ & $<8$ & $<8$ & $<8$ & $<4$ & $<8$ & $<4$ & $<8$ \\
\hline Tetramethylsuccinonitrile & $\mu \mathrm{g} / \mathrm{L}$ & U.S. & & & $<4$ & $<4$ & $<4$ & $<8$ & $<8$ & $<8$ & $<4$ & $<8$ & $<4$ & $<8$ \\
\hline \begin{tabular}{|l|} 
Tetramethyl thiourea \\
\end{tabular} & $\mu \mathrm{g} / \mathrm{L}$ & U.S. & & & $<4$ & $<4$ & $<4$ & $<8$ & $<8$ & $<8$ & $<4$ & $<8$ & $<4$ & $<8$ \\
\hline Tetramethylurea & $\mu \mathrm{g} / \mathrm{L}$ & U.S. & & & $<4$ & $<4$ & $<4$ & $<8$ & $<8$ & $<8$ & $<4$ & $<8$ & $<4$ & $<8$ \\
\hline \begin{tabular}{|l|l|} 
Thymol \\
\end{tabular} & $\mu \mathrm{g} / \mathrm{L}$ & U.S. & & & $<4$ & $<4$ & $<4$ & $<8$ & $<8$ & $<8$ & $<4$ & $<8$ & $<4$ & $<8$ \\
\hline $1,3,5-$-Triallyl-1,3,5-triazine-2,4,6(1H,3H,5H)-trione & $\mu \mathrm{g} / \mathrm{L}$ & U.S. & & & $<4$ & $<4$ & $<4$ & $<8$ & $<8$ & $<8$ & $<4$ & $<8$ & $<4$ & $<8$ \\
\hline Tributylamine & $\mu \mathrm{g} / \mathrm{L}$ & U.S. & Trialkylamines 400 & SWEG & $<4$ & $<4$ & $<4$ & $<8$ & $<8$ & $<8$ & $<4$ & $<8$ & $<4$ & $<8$ \\
\hline Tributyl phosphate & $\mu \mathrm{g} / \mathrm{L}$ & U.S. & & & $<4$ & $<4$ & $<4$ & $<8$ & $<8$ & $<8$ & $<4$ & $<8$ & $<4$ & $<8$ \\
\hline Triethyl phosphate & $\mu \mathrm{g} / \mathrm{L}$ & U.S. & & & $<8$ & $<8$ & $<8$ & $<16$ & $<16$ & $<16$ & $<8$ & $<16$ & $<8$ & $<16$ \\
\hline 2,2,4-Trimethyl-1,3-pentanediol diisobutyrate & $\mu \mathrm{g} / \mathrm{L}$ & U.S. & & & $<8$ & $<8$ & $<8$ & $<16$ & $<16$ & $<16$ & $<8$ & $<16$ & $<8$ & $<16$ \\
\hline Tripropylene glycol monomethyl ether & $\mu \mathrm{g} / \mathrm{L}$ & U.S. & & & $<4$ & $<4$ & $<4$ & $<8$ & $<8$ & $<8$ & $<4$ & $<8$ & $<4$ & $<8$ \\
\hline Undecanoic acid & $\mu \mathrm{g} / \mathrm{L}$ & U.S. & & & $<24$ & $<24$ & $<24$ & $<48$ & $<48$ & $<48$ & $<24$ & $<48$ & $<24$ & $<48$ \\
\hline \begin{tabular}{|l|} 
2-Undecanone \\
\end{tabular} & $\mu \mathrm{g} / \mathrm{L}$ & U.S. & & & $<4$ & $<4$ & $<4$ & $<8$ & $<8$ & $<8$ & $<4$ & $<8$ & $<4$ & $<8$ \\
\hline Valeric acid (Pentanoic acid) & $\mu \mathrm{g} / \mathrm{L}$ & U.S. & & & $<24$ & $<24$ & $<24$ & $<48$ & $<48$ & $<48$ & $<24$ & $<48$ & $<24$ & $<48$ \\
\hline Vanillin & $\mu \mathrm{g} / \mathrm{L}$ & U.S. & & & $<8$ & $<8$ & $<8$ & $<16$ & $<16$ & $<16$ & $<8$ & $<16$ & $<8$ & $<16$ \\
\hline & & & & & & & & & & & & & & \\
\hline Alcohols (DAI/GC/M S) & & & & & & & & & & & & & & \\
\hline 1-Butanol & $\mu \mathrm{g} / \mathrm{L}$ & U.S. & & & $<100$ & $<100$ & $<100$ & $<100$ & $<100$ & $<100$ & $<100$ & $<100$ & $<100$ & $<100$ \\
\hline 2-Butanol & $\mu \mathrm{g} / \mathrm{L}$ & U.S. & & & $<100$ & $<100$ & $<100$ & $<100$ & $<100$ & $<100$ & $<100$ & $<100$ & $<100$ & $<100$ \\
\hline \begin{tabular}{|l|} 
Ethanol \\
\end{tabular} & $\mu \mathrm{g} / \mathrm{L}$ & U.S. & & & $<100$ & $<100$ & $<100$ & $<100$ & $<100$ & $<100$ & $\angle 100$ & $<100$ & $<100$ & $<100$ \\
\hline Methanol & $\mu \mathrm{g} / \mathrm{L}$ & U.S. & & & $<100$ & $<100$ & $<100$ & $<100$ & $<100$ & $<100$ & $<100$ & $<100$ & $<100$ & $<100$ \\
\hline 2-Methyl-1-butanol & $\mu \mathrm{g} / \mathrm{L}$ & U.S. & & & $<100$ & $<100$ & $<100$ & $<100$ & $<100$ & $<100$ & $<100$ & $<100$ & $<100$ & $<100$ \\
\hline \begin{tabular}{|l|} 
2-Methyl-2-butanol \\
\end{tabular} & $\mu \mathrm{g} / \mathrm{L}$ & U.S. & & & $<100$ & $<100$ & $<100$ & $<100$ & $<100$ & $<100$ & $<100$ & $<100$ & $<100$ & $<100$ \\
\hline 3-Methyl-1-butanol (Isopentanol) & $\mu \mathrm{g} / \mathrm{L}$ & U.S. & & & $<100$ & $<100$ & $<100$ & $<100$ & $<100$ & $<100$ & $<100$ & $<100$ & $<100$ & $<100$ \\
\hline 2-Methyl-1-propanol & $\mu \mathrm{g} / \mathrm{L}$ & U.S. & & & $<100$ & $<100$ & $<100$ & $<100$ & $<100$ & $<100$ & $<100$ & $<100$ & $<100$ & $<100$ \\
\hline 2-Methyl-2-propanol & $\mu \mathrm{g} / \mathrm{L}$ & U.S. & & & $<100$ & $<100$ & $<100$ & $<100$ & $<100$ & $<100$ & $<100$ & $<100$ & $<100$ & $<100$ \\
\hline 1-Pentanol (Amyl alcohol) & $\mu \mathrm{g} / \mathrm{L}$ & U.S. & & & $<100$ & $<100$ & $<100$ & $<100$ & $<100$ & $<100$ & $<100$ & $<100$ & $<100$ & $<100$ \\
\hline 2-Pentanol (sec-A myl alcohol) & $\mu \mathrm{g} / \mathrm{L}$ & U.S. & & & $<100$ & $<100$ & $<100$ & $<100$ & $<100$ & $<100$ & $<100$ & $<100$ & $<100$ & $<100$ \\
\hline 3-Pentanol & $\mu \mathrm{g} / \mathrm{L}$ & U.S. & & & $<100$ & $<100$ & $<100$ & $<100$ & $<100$ & $<100$ & $<100$ & $<100$ & $<100$ & $<100$ \\
\hline 1-Propanol & $\mu \mathrm{g} / \mathrm{L}$ & U.S. & & & $<100$ & $<100$ & $<100$ & $<100$ & $<100$ & $<100$ & $<100$ & $<100$ & $<100$ & $<100$ \\
\hline 2-Propanol (Isopropanol) & $\mu \mathrm{g} / \mathrm{L}$ & U.S. & & & $<100$ & $<100$ & $<100$ & $<100$ & $<100$ & $<100$ & $<100$ & $<100$ & $<100$ & $<100$ \\
\hline & & & & & & & & & & & & & & \\
\hline Glycols (DAI/GC/MS) & & & & & & & & & & & & & & \\
\hline 1,2-Ethanediol (Ethylene glycol) & $\mu \mathrm{g} / \mathrm{L}$ & U.S. & $12000 / 14000$ & MORD/EPA HA & $<1000$ & $<1000$ & $<1000$ & $<1000$ & $<1000$ & $<1000$ & $<1000$ & $<1000$ & $<1000$ & $<1000$ \\
\hline 1,2-Propanediol (Propylene glycol) & $\mu \mathrm{g} / \mathrm{L}$ & U.S. & & & $<500$ & $<500$ & $<500$ & $<500$ & $<500$ & $<500$ & $<500$ & $<500$ & $<500$ & $<500$ \\
\hline & & & & & & & & & & & & & & \\
\hline \begin{tabular}{|l} 
Glycerol (LC/M S/MS) \\
\end{tabular} & & & & & & & & & & & & & & \\
\hline Glycerol (1,2,3-Propanetriol) & $\mu \mathrm{g} / \mathrm{L}$ & U.S. & & & NA & NA & $\mathrm{NA}$ & NA & $\mathrm{NA}$ & $\mathrm{NA}$ & $\mathrm{NA}$ & $\mathrm{NA}$ & $\mathrm{NA}$ & $<300$ \\
\hline Silanols (LC/M S/MS) (R \& D Method -NIST trace & ble stan & dard not avail & lable) & & & 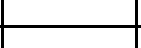 & 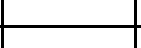 & & & & & & & \\
\hline $\begin{array}{l}\text { Dimethylsilanediol (DMSD) } \\
\end{array}$ & $\mu \mathrm{g} / \mathrm{L}$ & U.S. & & & $\mathrm{NA}$ & NA & NA & $\mathrm{NA}$ & $\mathrm{NA}$ & $\mathrm{NA}$ & $\mathrm{NA}$ & NA & NA & $<400$ \\
\hline & & & & & & & & & & & & & & \\
\hline \begin{tabular}{|l|} 
Carboxylates (CE) \\
\end{tabular} & & & & & & & & & & & & & & \\
\hline \begin{tabular}{|l|} 
Acetate \\
\end{tabular} & $\mu \mathrm{g} / \mathrm{L}$ & U.S. & & & $<125$ & $<125$ & $<125$ & $<125$ & $<125$ & $<125$ & $<125$ & $<125$ & $<125$ & $<125$ \\
\hline Formate & $\mu \mathrm{g} / \mathrm{L}$ & U.S. & $2,500,000$ & SWEG & $<125$ & $<125$ & $<125$ & $<125$ & $<125$ & $<125$ & $<125$ & $<125$ & $<125$ & $<125$ \\
\hline \begin{tabular}{|l} 
Glycolate \\
\end{tabular} & $\mu \mathrm{g} / \mathrm{L}$ & U.S. & & & $<125$ & $<125$ & $<125$ & $<125$ & $<125$ & $<125$ & $<125$ & $<125$ & $<125$ & $<125$ \\
\hline \begin{tabular}{|l|} 
Glyoxylate \\
\end{tabular} & $\mu \mathrm{g} / \mathrm{L}$ & U.S. & & & $<125$ & $<125$ & $<125$ & $<125$ & $<125$ & $<125$ & $<125$ & $<125$ & $<125$ & $<125$ \\
\hline \begin{tabular}{|l} 
Lactate \\
\end{tabular} & $\frac{\mu g / L}{\mu g}$ & U.S. & & & $<1000$ & $<1000$ & $<1000$ & $<1000$ & $<1000$ & $<1000$ & $<1000$ & $<1000$ & $<1000$ & $<1000$ \\
\hline Oxalate & $\mu \mathrm{g} / \mathrm{L}$ & U.S. & & & $<125$ & $<125$ & MI & $<125$ & $<125$ & $<125$ & $<125$ & $<125$ & $<125$ & $<125$ \\
\hline Propionate & $\mu \mathrm{g} / \mathrm{L}$ & U.S. & & & $<125$ & $<125$ & $<125$ & $<125$ & $<125$ & $<125$ & $<125$ & $<125$ & $<125$ & $<125$ \\
\hline & & & & & & & & & & & & & & \\
\hline
\end{tabular}


Appendix 1. ISS SRV-K Potable Water (Regenerated) Summary for Expeditions 21 through 25

\begin{tabular}{|c|c|c|c|c|c|c|c|c|c|c|c|c|c|c|}
\hline Mission & & & & & ISS ULF3/Exp. 21 & ISS 20A & Exp. 22 & Soyuz 20 & Exp. 22 & Soyuz 21/Exp. 23 & & SS ULF4/Exp. 2 & & \begin{tabular}{|l|} 
Soyuz 23/Exp. 25 \\
\end{tabular} \\
\hline Sample Location & & & Potable Water & & SRV-K Hot & SRV-K Warm & SRV-K Hot & SRV-K Warm & SRV-K Hot & SRV-K Hot & SRV-K Warm & SRV-K Warm & SRV-K Hot & SRV-K Warm \\
\hline \begin{tabular}{|l} 
Sample Description \\
Sample Date \\
Analysis/Sample ID
\end{tabular} & Units & $\begin{array}{c}\text { Test } \\
\text { Conducted } \\
\text { by }\end{array}$ & $\begin{array}{l}\text { Maximum } \\
\text { Contaminant } \\
\text { Level } \\
\text { (MCL) }\end{array}$ & $\begin{array}{c}\text { Maximum } \\
\text { Contaminant } \\
\text { Level } \\
\text { Source }\end{array}$ & $\begin{array}{c}\text { Potable Water } \\
11 / 10 / 2009 \\
20091130011\end{array}$ & $\begin{array}{c}\text { Potable Water } \\
1 / 6 / 2010 \\
20100222006\end{array}$ & $\begin{array}{c}\text { Potable Water } \\
2 / 3 / 2010 \\
20100222008\end{array}$ & $\begin{array}{c}\text { Potable Water } \\
3 / 3 / 2010 \\
20100326002\end{array}$ & $\begin{array}{c}\text { Potable Water } \\
3 / 3 / 2010 \\
20100326003\end{array}$ & $\begin{array}{l}\text { Potable Water } \\
3 / 31 / 2010 \\
20100603001\end{array}$ & $\begin{array}{c}\text { Potable Water } \\
4 / 26 / 2010 \\
20100527010\end{array}$ & $\left|\begin{array}{c}\text { Potable Water } \\
5 / 18 / 2010 \\
20100527011\end{array}\right|$ & $\mid \begin{array}{c}\text { Potable Water } \\
5 / 18 / 2010 \\
20100527012\end{array}$ & $\begin{array}{l}\text { Potable Water } \\
11 / 23 / 2010 \\
20101129003\end{array}$ \\
\hline \begin{tabular}{|l|} 
Aldehydes \\
\end{tabular} & & & & & & & & & & & & & & \\
\hline \begin{tabular}{|l} 
Formaldehyde \\
\end{tabular} & $\mu \mathrm{g} / \mathrm{L}$ & U.S. & $12,000 / 1,000$ & SWEG/EPA HA & $<2$ & $<2$ & $<2$ & $<2$ & $<2$ & 6 & 3 & 2 & 5 & $<5$ \\
\hline Amines (CE) & & & & & & & & & & & & & & \\
\hline Ethylamine & $\mu \mathrm{g} / \mathrm{L}$ & U.S. & Monoalkylamines 2000 & SWEG & $<125$ & $<125$ & $<125$ & $<125$ & $<125$ & $<125$ & $<125$ & $<125$ & $<125$ & $<125$ \\
\hline \begin{tabular}{|l|} 
Methylamine \\
\end{tabular} & $\mu \mathrm{g} / \mathrm{L}$ & U.S. & Monoalkylamines 2000 & SWEG & $<125$ & $<125$ & $<125$ & $<125$ & $<125$ & $<125$ & $<125$ & $<125$ & $<125$ & $<125$ \\
\hline \begin{tabular}{|l} 
n-Propylamine \\
\end{tabular} & $\mu \mathrm{g} / \mathrm{L}$ & U.S. & Monoalkylamines 2000 & SWEG & $<125$ & $<125$ & $<125$ & $<125$ & $<125$ & $<125$ & $<125$ & $<125$ & $<125$ & $<125$ \\
\hline \begin{tabular}{|l} 
Trimethylamine \\
\end{tabular} & $\mu \mathrm{g} / \mathrm{L}$ & U.S. & Trialkylamines 400 & SWEG & $<125$ & $<125$ & $<125$ & $<125$ & $<125$ & $<125$ & $<125$ & $<125$ & $<125$ & $<125$ \\
\hline Non-volatiles (LC/UV-VIS) & & & & & & & & & & & & & & \\
\hline Urea & $\mu \mathrm{g} / \mathrm{L}$ & U.S. & & & $<800$ & $<800$ & $<800$ & $<800$ & $<800$ & $<800$ & $<800$ & $<800$ & $<800$ & $<800$ \\
\hline Caprolactam & $\mu \mathrm{g} / \mathrm{L}$ & U.S. & 100,000 & SWEG & $<4$ & $<4$ & $<4$ & $<8$ & $<8$ & $<8$ & $<4$ & $<8$ & $<8$ & $<16$ \\
\hline & & & & & & & & & & & & & & \\
\hline \begin{tabular}{|l|} 
Organic Carbon Recovery \\
\end{tabular} & percent & U.S. & & & 9.26 & 1.92 & 0.53 & 0.00 & 0.08 & 0.51 & 0.68 & 0.56 & 3.08 & 0.00 \\
\hline Unaccounted Organic Carbon & $\mathrm{mg} / \mathrm{L}$ & U.S. & & & 0.15 & 0.20 & 1.31 & 0.21 & 0.19 & 0.50 & 0.17 & 0.27 & 0.26 & 0.38 \\
\hline
\end{tabular}




\section{Appendix 2. ISS SVO-ZV Potable Water Summary for Expeditions 21 through 25}

\begin{tabular}{|c|c|c|c|c|c|c|c|c|c|c|c|c|}
\hline \multirow{3}{*}{\begin{tabular}{|l} 
Mission \\
Sample Location \\
Sample Description \\
Sample Date \\
Analysis/Sample ID \\
\end{tabular}} & \multirow[b]{3}{*}{ Units } & \multirow[b]{3}{*}{$\begin{array}{c}\text { Test } \\
\text { Conducted } \\
\text { by }\end{array}$} & \multirow{3}{*}{$\begin{array}{c}\text { Potable W ater } \\
\text { Maximum } \\
\text { Contaminant } \\
\text { Level } \\
\text { (MCL) } \\
\end{array}$} & \multirow{3}{*}{$\begin{array}{c}\text { Maximum } \\
\text { Contaminant } \\
\text { Level } \\
\text { Source } \\
\end{array}$} & \multicolumn{2}{|c|}{\begin{tabular}{l|l|l} 
Soyuz 19/Exp. 21 & ISS ULF3/Exp. \\
\end{tabular}} & \multicolumn{2}{|c|}{ ISS 20A/Exp. 22} & \multirow{2}{*}{\begin{tabular}{|c|} 
Soyuz 20/Exp. 22 \\
SVO-ZV
\end{tabular}} & \multirow{2}{*}{\begin{tabular}{|c|} 
Soyuz 21/Exp. 23 \\
SVO-ZV
\end{tabular}} & \multicolumn{2}{|c|}{ ISS ULF4/Exp. 23} \\
\hline & & & & & SVO-ZV & SVO-ZV & SVO-ZV & SVO-ZV & & & SVO-ZV & SVO-ZV \\
\hline & & & & & $\begin{array}{c}\text { Potable W ater } \\
10 / 20 / 2009 \\
20091214003\end{array}$ & $\begin{array}{c}\text { Potable Water } \\
11 / 10 / 2009 \\
20091130010\end{array}$ & $\begin{array}{c}\text { Potable } W \text { ater } \\
1 / 6 / 2010 \\
20100222005\end{array}$ & \begin{tabular}{|c|} 
Potable $W$ ater \\
$2 / 3 / 2010$ \\
20100222007
\end{tabular} & $\begin{array}{l}\text { Potable W ater } \\
3 / 3 / 2010 \\
20100326001\end{array}$ & $\begin{array}{c}\text { Potable W ater } \\
3 / 31 / 2010 \\
20100603002\end{array}$ & \begin{tabular}{|c|} 
Potable W ater \\
$4 / 26 / 2010$ \\
20100527009
\end{tabular} & $\begin{array}{c}\text { Potable W ater } \\
5 / 18 / 2010 \\
20100527013\end{array}$ \\
\hline \multicolumn{13}{|l|}{ Physical Characteristics } \\
\hline $\mathrm{pH}$ & $\mathrm{pH}$ units & U.S. & $5.5-9.0$ & MORD & 7.00 & 7.00 & 6.69 & 6.96 & 7.14 & 6.50 & 6.29 & 6.46 \\
\hline Conductivity & $\mu \mathrm{S} / \mathrm{cm}$ & U.S. & & & 334 & 304 & 362 & 362 & 363 & 300 & 251 & 269 \\
\hline Turbidity & NTU & U.S. & $1.5^{*}$ & MORD & 1.6 & 0.3 & 2.0 & 3.8 & 3.5 & NA & 1.1 & 1.3 \\
\hline Total Dissolved Solids & $\mathrm{mg} / \mathrm{L}$ & U.S. & $100\left(1,000^{*}\right)$ & MORD & $\mathrm{NA}$ & 180 & 215 & 222 & $\mathrm{NA}$ & $\mathrm{NA}$ & $\mathrm{NA}$ & 141 \\
\hline \multicolumn{12}{|l|}{ Iodine (LCV) } & \\
\hline Total I & $\mathrm{mg} / \mathrm{L}$ & U.S. & 0.05 & MORD & $<0.05$ & $<0.05$ & $<0.05$ & $<0.05$ & $<0.05$ & $<0.05$ & $<0.05$ & $<0.05$ \\
\hline \multicolumn{13}{|l|}{\begin{tabular}{|l|} 
Anions (IC/ISE) \\
\end{tabular}} \\
\hline Bromide & $\mathrm{mg} / \mathrm{L}$ & U.S. & & & $<0.5$ & $<0.5$ & $<0.5$ & $<0.5$ & $<0.5$ & $<0.5$ & $<0.5$ & $<0.5$ \\
\hline Chloride & $\mathrm{mg} / \mathrm{L}$ & U.S. & 250 & MORD & 9.75 & 8.91 & 9.79 & 9.81 & 10.1 & 10.2 & 8.61 & 8.68 \\
\hline Fluoride & $\mathrm{mg} / \mathrm{L}$ & U.S. & $1.5 / 4$ & MORD/EPA & $<0.1$ & $<0.1$ & $<0.1$ & $<0.1$ & $<0.1$ & 0.1 & $<0.1$ & $<0.1$ \\
\hline Nitrate as Nitrogen (NO3-N) & $\mathrm{mg} / \mathrm{L}$ & U.S. & 10 & MORD/EPA & 0.33 & $<0.11$ & 0.45 & 0.49 & 0.57 & 0.62 & $<0.11$ & $<0.11$ \\
\hline Nitrite as Nitrogen (NO2-N) & $\mathrm{mg} / \mathrm{L}$ & U.S. & 1 & EPA & $\mathrm{NA}$ & $\mathrm{NA}$ & $\mathrm{NA}$ & $\mathrm{NA}$ & $\mathrm{NA}$ & $\mathrm{NA}$ & $\mathrm{NA}$ & $\mathrm{NA}$ \\
\hline \begin{tabular}{|l} 
Phosphate as P (PO4-P) \\
\end{tabular} & $\mathrm{mg} / \mathrm{L}$ & U.S. & & & $<0.24$ & $<0.24$ & $<0.24$ & $<0.24$ & $<0.24$ & $<0.24$ & $<0.24$ & $<0.24$ \\
\hline Sulfate & $\mathrm{mg} / \mathrm{L}$ & U.S. & 250 & MORD & 38 & 28.4 & 40.7 & 40.8 & 41.8 & 42.1 & 25.5 & 26.3 \\
\hline & & & & & & & & & & & & \\
\hline \multicolumn{13}{|l|}{ Cations (IC) } \\
\hline Ammonia as Nitrogen (NH3-N) & $\mathrm{mg} / \mathrm{L}$ & U.S. & $2 / 1$ & MORD/SWEG & $<0.002$ & $<0.002$ & $<0.002$ & $<0.002$ & $<0.002$ & $<0.002$ & $<0.002$ & $<0.002$ \\
\hline Lithium & $\mathrm{mg} / \mathrm{L}$ & U.S. & & & $<0.002$ & $<0.002$ & $<0.002$ & $<0.002$ & $<0.002$ & $<0.002$ & $<0.002$ & $<0.002$ \\
\hline & & & & & & & & & & & & \\
\hline \multicolumn{13}{|l|}{ Metals (ICP/MS) } \\
\hline Calcium & $\mathrm{mg} / \mathrm{L}$ & U.S. & 100 & MORD & 44.4 & 39.5 & 48.6 & 49.5 & 47.4 & 49.9 & 33.1 & 32.9 \\
\hline Magnesium & $\mathrm{mg} / \mathrm{L}$ & U.S. & 50 & MORD & 11.1 & 10.1 & 11 & 11.1 & 11.2 & 12.1 & 8.65 & 8.19 \\
\hline Potassium & $\mathrm{mg} / \mathrm{L}$ & U.S. & & & 2.29 & 3.01 & 2.05 & 2.1 & 2.27 & 2.2 & 2.1 & 2.04 \\
\hline \begin{tabular}{|l|} 
Sodium \\
\end{tabular} & $\mathrm{mg} / \mathrm{L}$ & U.S. & & & 6.37 & 7.51 & 6.33 & 6.34 & 6.09 & 6.28 & 9.62 & 8.98 \\
\hline Aluminum & $\mu \mathrm{g} / \mathrm{L}$ & U.S. & & & 54 & 103 & 36 & 41 & 44 & 105 & 170 & 166 \\
\hline Antimony & $\mu \mathrm{g} / \mathrm{L}$ & U.S. & 6 & EPA & $<2$ & $<2$ & $<2$ & $<2$ & $<2$ & $<2$ & $<2$ & $<2$ \\
\hline Arsenic & $\mu \mathrm{g} / \mathrm{L}$ & U.S. & 10 & MORD/EPA & $<1$ & $<1$ & $<1$ & $<1$ & $<1$ & $<1$ & $<1$ & $<1$ \\
\hline Barium & $\mu \mathrm{g} / \mathrm{L}$ & U.S. & $1,000 / 10,000$ & MORD/SWEG & 25 & 7 & 33 & 33 & 36 & 36 & 6 & 6 \\
\hline Beryllium & $\mu \mathrm{g} / \mathrm{L}$ & U.S. & 4 & EPA & $<1$ & $<1$ & $<1$ & $<1$ & $<1$ & $<1$ & $<1$ & $<1$ \\
\hline Cadmium & $\mu \mathrm{g} / \mathrm{L}$ & U.S. & $5 / 22$ & MORD/SWEG & $<1$ & 3 & $<1$ & $<1$ & $<1$ & $<1$ & 1 & 1 \\
\hline Chromium & $\mu \mathrm{g} / \mathrm{L}$ & U.S. & 100 & MORD/EPA & $<5$ & $<5$ & $<5$ & $<5$ & $<5$ & $<5$ & $<5$ & $<5$ \\
\hline Cobalt & $\mu \mathrm{g} / \mathrm{L}$ & U.S. & & & $<1$ & $<1$ & $<1$ & $<1$ & $<1$ & $<1$ & $<1$ & $<1$ \\
\hline \begin{tabular}{|l|} 
Copper \\
\end{tabular} & $\mu \mathrm{g} / \mathrm{L}$ & U.S. & $1,000 / 1,300$ & MORD/EPA & 2 & $<1$ & 4 & 4 & 4 & 4 & 2 & 1 \\
\hline Iron & $\mu \mathrm{g} / \mathrm{L}$ & U.S. & 300 & MORD & 57 & 76 & 54 & 68 & 102 & 60 & 50 & 56 \\
\hline Lead & $\mu \mathrm{g} / \mathrm{L}$ & U.S. & $50 / 15$ & MORD/EPA & $<1$ & $<1$ & $<1$ & $<1$ & $<1$ & $<1$ & $<1$ & $<1$ \\
\hline \begin{tabular}{|l} 
Manganese \\
\end{tabular} & $\mu \mathrm{g} / \mathrm{L}$ & U.S. & $50 / 300$ & MORD/SWEG & 33 & 36 & 35 & 35 & 35 & 35 & 27 & 27 \\
\hline Mercury & $\mu \mathrm{g} / \mathrm{L}$ & U.S. & 2 & MORD/EPA & $<0.5$ & $<0.5$ & $<0.5$ & $<0.5$ & $<0.5$ & $<0.5$ & $<0.5$ & $<0.5$ \\
\hline \begin{tabular}{|l|} 
Molybdenum \\
\end{tabular} & $\mu \mathrm{g} / \mathrm{L}$ & U.S. & 40 & EPA HA & $<1$ & $<1$ & $<1$ & $<1$ & $<1$ & $<1$ & $<1$ & $<1$ \\
\hline Nickel & $\mu \mathrm{g} / \mathrm{L}$ & U.S. & $100 / 300$ & MORD/SWEG & 4 & 3 & 24 & 5 & 5 & 5 & 2 & 1 \\
\hline Selenium & $\mu \mathrm{g} / \mathrm{L}$ & U.S. & $10 / 50$ & MORD/EPA & $<1$ & $<1$ & $<1$ & $<1$ & $<1$ & $<1$ & $<1$ & $<1$ \\
\hline \begin{tabular}{|l|} 
Silver \\
\end{tabular} & $\mu \mathrm{g} / \mathrm{L}$ & U.S. & $500 / 400$ & MORD/SWEG & 111 & 28 & 173 & 405 & 276 & 187 & 132 & 160 \\
\hline Silver, Dissolved & $\mu \mathrm{g} / \mathrm{L}$ & U.S. & & & 81 & 18 & 56 & 110 & 69 & 90 & 86 & 125 \\
\hline Zinc & $\mu \mathrm{g} / \mathrm{L}$ & U.S. & $5,000 / 2,000$ & MORD/SWEG & 30 & 8 & 132 & 27 & 24 & 29 & 7 & 5 \\
\hline & & & & & & & & & & & & \\
\hline
\end{tabular}




\section{Appendix 2. ISS SVO-ZV Potable Water Summary for Expeditions 21 through 25}

\begin{tabular}{|c|c|c|c|c|c|c|c|c|c|c|c|c|}
\hline Mission & & & & & Soyuz 19/Exp. 21 & SS ULF3/Exp. 21 & ISS 20A & $\sqrt{\operatorname{Exp} .22}$ & Soyuz 20/Exp. 22 & Soyuz 21/Exp. 23 & ISS ULF4 & 4/Exp. 23 \\
\hline Sample Location & & & $\begin{array}{l}\text { Potable W ater } \\
\text { Maximum }\end{array}$ & Maximum & SVO-ZV & SVO-ZV & SVO-ZV & SVO-ZV & SVO-ZV & SVO-ZV & SVO-ZV & SVO-ZV \\
\hline $\begin{array}{l}\text { Sample Description } \\
\text { Sample Date } \\
\text { Analysis/Sample ID }\end{array}$ & Units & $\begin{array}{c}\text { Test } \\
\text { Conducted } \\
\text { by }\end{array}$ & $\begin{array}{l}\text { Contaminant } \\
\text { Level } \\
\text { (MCL) }\end{array}$ & $\begin{array}{c}\text { Contaminant } \\
\text { Level } \\
\text { Source }\end{array}$ & $\begin{array}{l}\text { Potable W ater } \\
10 / 20 / 2009 \\
20091214003\end{array}$ & $\begin{array}{c}\text { Potable Water } \\
11 / 10 / 2009 \\
20091130010\end{array}$ & $\begin{array}{c}\text { Potable } W \text { ater } \\
1 / 6 / 2010 \\
20100222005\end{array}$ & \begin{tabular}{|c|} 
Potable W ater \\
$2 / 3 / 2010$ \\
20100222007 \\
\end{tabular} & $\begin{array}{l}\text { Potable W ater } \\
3 / 3 / 2010 \\
20100326001\end{array}$ & $\begin{array}{c}\text { Potable W ater } \\
3 / 31 / 2010 \\
20100603002\end{array}$ & \begin{tabular}{|c|} 
Potable W ater \\
$4 / 26 / 2010$ \\
20100527009
\end{tabular} & \begin{tabular}{|c|} 
Potable W ater \\
$5 / 18 / 2010$ \\
20100527013
\end{tabular} \\
\hline Silicon (ICP/MS) & & & & & & & & & & & & \\
\hline Silicon (ICP/MS) & $\mu \mathrm{q} / \mathrm{L}$ & U.S. & & & $\mathrm{NA}$ & $\mathrm{NA}$ & $\mathrm{NA}$ & $\mathrm{NA}$ & $\mathrm{NA}$ & $\mathrm{NA}$ & $\mathrm{NA}$ & $\mathrm{NA}$ \\
\hline & & & & & & & & & & & & \\
\hline Total Organic Carbon (Sievers) & & & & & & & & & & & & \\
\hline Total Inorganic Carbon & $\mathrm{mg} / \mathrm{L}$ & U.S. & & & 31.5 & 29.7 & 31.7 & 32.9 & 31.4 & 31.9 & 25.8 & 25.6 \\
\hline Total Organic Carbon & $\mathrm{mg} / \mathrm{L}$ & U.S. & $20^{* *}$ & MORD & 2.70 & 0.36 & 3.38 & 3.26 & 3.48 & 3.53 & 0.48 & 0.48 \\
\hline & & & & & & & & & & & & \\
\hline Volatile Organics & & & & & & & & & & & & \\
\hline Acetone & $\mu \mathrm{g} / \mathrm{L}$ & U.S. & 15,000 & SWEG & $<2$ & $<2$ & $<2$ & $<2$ & $<2$ & $<6$ & $<2$ & $<2$ \\
\hline \begin{tabular}{|l|} 
Acryloniltrile \\
\end{tabular} & $\mu \mathrm{g} / \mathrm{L}$ & U.S. & & & $<2$ & $<2$ & $<2$ & $<2$ & $<2$ & $<6$ & $<2$ & $<2$ \\
\hline 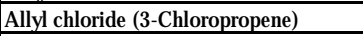 & $\mu \mathrm{g} / \mathrm{L}$ & U.S. & & & $<2$ & $<2$ & $<2$ & $<2$ & $<2$ & $<6$ & $<2$ & $<2$ \\
\hline Benzene & $\mu \mathrm{g} / \mathrm{L}$ & U.S. & 5 & EPA & $<0.4$ & $<0.4$ & $<0.4$ & $<0.4$ & $<0.4$ & $<1.2$ & $<0.4$ & $<0.4$ \\
\hline Bromobenzene & $\mu \mathrm{g} / \mathrm{L}$ & U.S. & & & $<0.4$ & $<0.4$ & $<0.4$ & $<0.4$ & $<0.4$ & $<1.2$ & $<0.4$ & $<0.4$ \\
\hline Bromochloromethane & $\mu \mathrm{g} / \mathrm{L}$ & U.S. & 90 & EPA HA & $<4$ & $<4$ & $<4$ & $<4$ & $<4$ & $<12$ & $<4$ & $<4$ \\
\hline Bromodichloromethane & $\mu \mathrm{g} / \mathrm{L}$ & U.S. & THM 80 & EPA & 1.9 & $<0.4$ & 3 & 3.1 & 3.5 & 2.6 & $<0.4$ & $<0.4$ \\
\hline Bromoform & $\mu \mathrm{g} / \mathrm{L}$ & U.S. & THM 80 & EPA & $<2$ & $<2$ & $<2$ & $<2$ & $<2$ & $<6$ & $<2$ & $<2$ \\
\hline Bromomethane & $\mu \mathrm{g} / \mathrm{L}$ & U.S. & 10 & EPA HA & $<2$ & $<2$ & $<2$ & $<2$ & $<2$ & $<6$ & $<2$ & $<2$ \\
\hline 2-Butanone (Methyl ethyl ketone) & $\mu \mathrm{g} / \mathrm{L}$ & U.S. & 4,000 & EPA HA & $<2$ & $<2$ & $<2$ & $<2$ & $<2$ & $<6$ & $<2$ & $<2$ \\
\hline n-Butylbenzene & $\mu \mathrm{g} / \mathrm{L}$ & U.S. & & & $<0.4$ & $<0.4$ & $<0.4$ & $<0.4$ & $<0.4$ & $<1.2$ & $<0.4$ & $<0.4$ \\
\hline sec-Butylbenzene & $\mu \mathrm{g} / \mathrm{L}$ & U.S. & & & $<0.4$ & $<0.4$ & $<0.4$ & $<0.4$ & $<0.4$ & $<1.2$ & $<0.4$ & $<0.4$ \\
\hline tert-Butylbenzene & $\mu \mathrm{g} / \mathrm{L}$ & U.S. & & & $<0.4$ & $<0.4$ & $<0.4$ & $<0.4$ & $<0.4$ & $<1.2$ & $<0.4$ & $<0.4$ \\
\hline Carbon disulfide & $\mu \mathrm{g} / \mathrm{L}$ & U.S. & & & $<2$ & $<2$ & $<2$ & $<2$ & $<2$ & $<6$ & $<2$ & $<2$ \\
\hline \begin{tabular}{|l|} 
Carbon tetrachloride \\
\end{tabular} & $\mu \mathrm{g} / \mathrm{L}$ & U.S. & 5 & EPA & $<0.4$ & $<0.4$ & $<0.4$ & $<0.4$ & $<0.4$ & $<1.2$ & $<0.4$ & $<0.4$ \\
\hline \begin{tabular}{|l|} 
Chloroacetonitrile \\
\end{tabular} & $\mu \mathrm{g} / \mathrm{L}$ & U.S. & & & $<10$ & $<10$ & $<10$ & $<10$ & $<10$ & $<30$ & $<10$ & $<10$ \\
\hline Chlorobenzene & $\mu \mathrm{g} / \mathrm{L}$ & U.S. & 100 & EPA & $<0.4$ & $<0.4$ & $<0.4$ & $<0.4$ & $<0.4$ & $<1.2$ & $<0.4$ & $<0.4$ \\
\hline 1-Chlorobutane (Butyl chloride) & $\mu \mathrm{g} / \mathrm{L}$ & U.S. & & & $<0.4$ & $<0.4$ & $<0.4$ & $<0.4$ & $<0.4$ & $<1.2$ & $<0.4$ & $<0.4$ \\
\hline Chloroethane & $\mu \mathrm{g} / \mathrm{L}$ & U.S. & & & $<2$ & $<2$ & $<2$ & $<2$ & $<2$ & $<6$ & $<2$ & $<2$ \\
\hline \begin{tabular}{|l|} 
Chloroform \\
\end{tabular} & $\mu \mathrm{g} / \mathrm{L}$ & U.S. & $6,500 /$ THM 80 & SWEG/EPA & 51.9 & 1.6 & 67.9 & 76.8 & 89.2 & 47.8 & 1.4 & 1.6 \\
\hline Chloromethane & $\mu \mathrm{g} / \mathrm{L}$ & U.S. & 30 & EPA HA & $<2$ & $<2$ & $<2$ & $<2$ & $<2$ & $<6$ & $<2$ & $<2$ \\
\hline 2-Chlorotoluene & $\mu \mathrm{g} / \mathrm{L}$ & U.S. & 100 & EPA HA & $<0.4$ & $<0.4$ & $<0.4$ & $<0.4$ & $<0.4$ & $<1.2$ & $<0.4$ & $<0.4$ \\
\hline 4-Chlorotoluene & $\mu \mathrm{g} / \mathrm{L}$ & U.S. & 100 & EPA HA & $<0.4$ & $<0.4$ & $<0.4$ & $<0.4$ & $<0.4$ & $<1.2$ & $<0.4$ & $<0.4$ \\
\hline Dibromochloromethane & $\mu \mathrm{g} / \mathrm{L}$ & U.S. & THM 80 & EPA & $<0.4$ & $<0.4$ & $<0.4$ & $<0.4$ & $<0.4$ & $<1.2$ & $<0.4$ & $<0.4$ \\
\hline 1,2-Dibromo-3-chloropropane (DBCP) & $\mu \mathrm{g} / \mathrm{L}$ & U.S. & 0.2 & EPA & $<2$ & $<2$ & $<2$ & $<2$ & $<2$ & $<6$ & $<2$ & $<2$ \\
\hline 1,2-Dibromoethane (EDB) & $\mu \mathrm{g} / \mathrm{L}$ & U.S. & 0.05 & EPA & $<0.4$ & $<0.4$ & $<0.4$ & $<0.4$ & $<0.4$ & $<1.2$ & $<0.4$ & $<0.4$ \\
\hline Dibromomethane & $\mu \mathrm{g} / \mathrm{L}$ & U.S. & & & $<0.4$ & $<0.4$ & $<0.4$ & $<0.4$ & $<0.4$ & $<1.2$ & $<0.4$ & $<0.4$ \\
\hline 1,2-Dichlorobenzene & $\mu \mathrm{g} / \mathrm{L}$ & U.S. & 600 & EPA & $<0.4$ & $<0.4$ & $<0.4$ & $<0.4$ & $<0.4$ & $<1.2$ & $<0.4$ & $<0.4$ \\
\hline 1,3-Dichlorobenzene & $\mu \mathrm{g} / \mathrm{L}$ & U.S. & 600 & EPA HA & $<0.4$ & $<0.4$ & $<0.4$ & $<0.4$ & $<0.4$ & $<1.2$ & $<0.4$ & $<0.4$ \\
\hline 1,4-Dichlorobenzene & $\mu \mathrm{g} / \mathrm{L}$ & U.S. & 75 & EPA & $<0.4$ & $<0.4$ & $<0.4$ & $<0.4$ & $<0.4$ & $<1.2$ & $<0.4$ & $<0.4$ \\
\hline \begin{tabular}{|l|l|} 
trans-1,4-Dichloro-2-butene \\
\end{tabular} & $\mu \mathrm{g} / \mathrm{L}$ & U.S. & & & $<0.4$ & $<0.4$ & $<0.4$ & $<0.4$ & $<0.4$ & $<1.2$ & $<0.4$ & $<0.4$ \\
\hline \begin{tabular}{|l|} 
Dichlorodifluoromethane \\
\end{tabular} & $\mu \mathrm{g} / \mathrm{L}$ & U.S. & 1,000 & EPA HA & $<2$ & $<2$ & $<2$ & $<2$ & $<2$ & $<6$ & $<2$ & $<2$ \\
\hline 1,1-Dichloroethane & $\mu \mathrm{g} / \mathrm{L}$ & U.S. & & & $<0.4$ & $<0.4$ & $<0.4$ & $<0.4$ & $<0.4$ & $<1.2$ & $<0.4$ & $<0.4$ \\
\hline 1,2-Dichloroethane & $\mu \mathrm{g} / \mathrm{L}$ & U.S. & 5 & EPA & $<0.4$ & $<0.4$ & $<0.4$ & $<0.4$ & $<0.4$ & $<1.2$ & $<0.4$ & $<0.4$ \\
\hline 1,1-Dichloroethene & $\mu \mathrm{g} / \mathrm{L}$ & U.S. & 7 & EPA & $<0.4$ & $<0.4$ & $<0.4$ & $<0.4$ & $<0.4$ & $<1.2$ & $<0.4$ & $<0.4$ \\
\hline cis1,2-Dichloroethene & $\mu \mathrm{g} / \mathrm{L}$ & U.S. & 70 & EPA & $<0.4$ & $<0.4$ & $<0.4$ & $<0.4$ & $<0.4$ & $<1.2$ & $<0.4$ & $<0.4$ \\
\hline trans-1,2-Dichloroethene & $\mu \mathrm{g} / \mathrm{L}$ & U.S. & 100 & EPA & $<0.4$ & $<0.4$ & $<0.4$ & $<0.4$ & $<0.4$ & $<1.2$ & $<0.4$ & $<0.4$ \\
\hline 1,2-Dichloropropane & $\mu \mathrm{g} / \mathrm{L}$ & U.S. & 5 & EPA & $<0.4$ & $<0.4$ & $<0.4$ & $<0.4$ & $<0.4$ & $<1.2$ & $<0.4$ & $<0.4$ \\
\hline 1,3-Dichloropropane & $\mu \mathrm{g} / \mathrm{L}$ & U.S. & & & $<0.4$ & $<0.4$ & $<0.4$ & $<0.4$ & $<0.4$ & $<1.2$ & $<0.4$ & $<0.4$ \\
\hline 2,2-Dichloropropane & $\mu \mathrm{g} / \mathrm{L}$ & U.S. & & & $<0.4$ & $<0.4$ & $<0.4$ & $<0.4$ & $<0.4$ & $<1.2$ & $<0.4$ & $<0.4$ \\
\hline
\end{tabular}




\section{Appendix 2. ISS SVO-ZV Potable Water Summary for Expeditions 21 through 25}

\begin{tabular}{|c|c|c|c|c|c|c|c|c|c|c|c|c|}
\hline Mission & & & & & Soyuz 19/Exp. 21 & SS ULF3/Exp. 21 & ISS 20A & $\sqrt{\operatorname{Exp} .22}$ & Soyuz 20/Exp. 22 & \begin{tabular}{|l|} 
Soyuz 21/Exp. 23 \\
\end{tabular} & ISS ULF4 & $4 /$ Exp. 23 \\
\hline Sample Location & & & $\begin{array}{l}\text { Potable W ater } \\
\text { Maximum }\end{array}$ & Maximum & SVO-ZV & SVO-ZV & SVO-ZV & SVO-ZV & SVO-ZV & \begin{tabular}{|l|} 
SVO-ZV \\
\end{tabular} & \begin{tabular}{|l|} 
SVO-ZV \\
\end{tabular} & SVO-ZV \\
\hline $\begin{array}{l}\text { Sample Description } \\
\text { Sample Date } \\
\text { Analysis/Sample ID }\end{array}$ & Units & $\begin{array}{c}\text { Test } \\
\text { Conducted } \\
\text { by }\end{array}$ & $\begin{array}{l}\text { Contaminant } \\
\text { Level } \\
\text { (MCL) }\end{array}$ & $\begin{array}{l}\text { Contaminant } \\
\text { Level } \\
\text { Source }\end{array}$ & $\begin{array}{c}\text { Potable W ater } \\
10 / 20 / 2009 \\
20091214003\end{array}$ & $\begin{array}{c}\text { Potable Water } \\
11 / 10 / 2009 \\
20091130010\end{array}$ & $\begin{array}{c}\text { Potable } W \text { ater } \\
1 / 6 / 2010 \\
20100222005\end{array}$ & \begin{tabular}{|c|} 
Potable W ater \\
$2 / 3 / 2010$ \\
20100222007
\end{tabular} & $\begin{array}{l}\text { Potable W ater } \\
3 / 3 / 2010 \\
20100326001\end{array}$ & $\begin{array}{l}\text { Potable W ater } \\
3 / 31 / 2010 \\
20100603002\end{array}$ & \begin{tabular}{|c|} 
Potable W ater \\
$4 / 26 / 2010$ \\
20100527009
\end{tabular} & $\begin{array}{l}\text { Potable W ater } \\
5 / 18 / 2010 \\
20100527013\end{array}$ \\
\hline \begin{tabular}{|l|} 
Analysis/Sample ID \\
1,1-Dichloropropanone
\end{tabular} & $\mu \mathrm{g} / \mathrm{L}$ & U.S. & & & $<2$ & $<2$ & $<2$ & \begin{tabular}{|c|}
$20100 \angle 2 \angle 001$ \\
$<2$ \\
\end{tabular} & $<2$ & $<6$ & \begin{tabular}{|c|}
$2010002 / 009$ \\
$<2$ \\
\end{tabular} & \begin{tabular}{|c}
20100527013 \\
$<2$ \\
\end{tabular} \\
\hline 1,1-Dichloropropene & $\mu \mathrm{g} / \mathrm{L}$ & U.S. & & & $<0.4$ & $<0.4$ & $<0.4$ & $<0.4$ & $<0.4$ & $<1.2$ & $<0.4$ & $<0.4$ \\
\hline cis-1,3-Dichloropropene & $\mu \mathrm{g} / \mathrm{L}$ & U.S. & & & $<0.4$ & $<0.4$ & $<0.4$ & $<0.4$ & $<0.4$ & $<1.2$ & $<0.4$ & $<0.4$ \\
\hline trans-1,3-Dichloropropene & $\mu \mathrm{g} / \mathrm{L}$ & U.S. & & & $<2$ & $<2$ & $<2$ & $<2$ & $<2$ & $<6$ & $<2$ & $<2$ \\
\hline \begin{tabular}{|l|} 
Diethyl ether \\
\end{tabular} & $\mu \mathrm{g} / \mathrm{L}$ & U.S. & & & $<2$ & $<2$ & $<2$ & $<2$ & $<2$ & $<6$ & $<2$ & $<2$ \\
\hline Ethylbenzene & $\mu \mathrm{g} / \mathrm{L}$ & U.S. & 700 & EPA & $<0.4$ & $<0.4$ & $<0.4$ & $<0.4$ & $<0.4$ & $<1.2$ & $<0.4$ & $<0.4$ \\
\hline Ethyl methacrylate & $\mu \mathrm{g} / \mathrm{L}$ & U.S. & & & $<2$ & $<2$ & $<2$ & $<2$ & $<2$ & $<6$ & $<2$ & $<2$ \\
\hline Hexachlorobutadiene & $\mu \mathrm{g} / \mathrm{L}$ & U.S. & 1 & EPA HA & $<2$ & $<2$ & $<2$ & $<2$ & $<2$ & $<6$ & $<2$ & $<2$ \\
\hline Hexachloroethane & $\mu \mathrm{g} / \mathrm{L}$ & U.S. & 1 & EPA HA & $<2$ & $<2$ & $<2$ & $<2$ & $<2$ & $<6$ & $<2$ & $<2$ \\
\hline 2-Hexanone & $\mu \mathrm{g} / \mathrm{L}$ & U.S. & & & $<2$ & $<2$ & $<2$ & $<2$ & $<2$ & $<6$ & $<2$ & $<2$ \\
\hline Iodomethane & $\mu \mathrm{g} / \mathrm{L}$ & U.S. & & & $<2$ & $<2$ & $<2$ & $<2$ & $<2$ & $<6$ & $<2$ & $<2$ \\
\hline Isopropylbenzene (Cumene) & $\mu \mathrm{g} / \mathrm{L}$ & U.S. & 4,000 & EPA DWEL & $<0.4$ & $<0.4$ & $<0.4$ & $<0.4$ & $<0.4$ & $<1.2$ & $<0.4$ & $<0.4$ \\
\hline 4-Isopropyltoluene (Cymene) & $\mu \mathrm{g} / \mathrm{L}$ & U.S. & & & $<0.4$ & $<0.4$ & $<0.4$ & $<0.4$ & $<0.4$ & $<1.2$ & $<0.4$ & $<0.4$ \\
\hline Methacrylonitrile & $\mu \mathrm{g} / \mathrm{L}$ & U.S. & & & $<2$ & $<2$ & $<2$ & $<2$ & $<2$ & $<6$ & $<2$ & $<2$ \\
\hline Methyl acrylate & $\mu \mathrm{g} / \mathrm{L}$ & U.S. & & & $<2$ & $<2$ & $<2$ & $<2$ & $<2$ & $<6$ & $<2$ & $<2$ \\
\hline Methyl-t-butylether (MTBE) & $\mu \mathrm{g} / \mathrm{L}$ & U.S. & & & $<2$ & $<2$ & $<2$ & $<2$ & $<2$ & $<6$ & $<2$ & $<2$ \\
\hline Methylene chloride (Dichloromethane) & $\mu \mathrm{g} / \mathrm{L}$ & U.S. & $15,000 / 5$ & SWEG/EPA & $<0.4$ & $<0.4$ & $<0.4$ & $<0.4$ & $<0.4$ & $<1.2$ & $<0.4$ & $<0.4$ \\
\hline Methyl methacrylate & $\mu \mathrm{g} / \mathrm{L}$ & U.S. & & & $<2$ & $<2$ & $<2$ & $<2$ & $<2$ & $<6$ & $<2$ & $<2$ \\
\hline 4-Methyl-2-pentanone & $\mu \mathrm{g} / \mathrm{L}$ & U.S. & & & $<0.4$ & $<0.4$ & $<0.4$ & $<0.4$ & $<0.4$ & $<1.2$ & $<0.4$ & $<0.4$ \\
\hline Naphthalene & $\mu \mathrm{g} / \mathrm{L}$ & U.S. & 100 & EPA HA & $<0.4$ & $<0.4$ & $<0.4$ & $<0.4$ & $<0.4$ & $<1.2$ & $<0.4$ & $<0.4$ \\
\hline Nitrobenzene & $\mu \mathrm{g} / \mathrm{L}$ & U.S. & & & $<2$ & $<2$ & $<2$ & $<2$ & $<2$ & $<6$ & $<2$ & $<2$ \\
\hline 2-Nitropropane & $\mu \mathrm{g} / \mathrm{L}$ & U.S. & & & $<2$ & $<2$ & $<2$ & $<2$ & $<2$ & $<6$ & $<2$ & $<2$ \\
\hline Pentachloroethane & $\mu \mathrm{g} / \mathrm{L}$ & U.S. & & & $<2$ & $<2$ & $<2$ & $<2$ & $<2$ & $<6$ & $<2$ & $<2$ \\
\hline Propionitrile (Ethyl cyanide) & $\mu \mathrm{g} / \mathrm{L}$ & U.S. & & & $<10$ & $<10$ & $<10$ & $<10$ & $<10$ & $<30$ & $<10$ & $<10$ \\
\hline n-Propylbenzene & $\mu \mathrm{g} / \mathrm{L}$ & U.S. & & & $<0.4$ & $<0.4$ & $<0.4$ & $<0.4$ & $<0.4$ & $<1.2$ & $<0.4$ & $<0.4$ \\
\hline Styrene & $\mu \mathrm{g} / \mathrm{L}$ & U.S. & 100 & EPA & $<0.4$ & $<0.4$ & $<0.4$ & $<0.4$ & $<0.4$ & $<1.2$ & $<0.4$ & $<0.4$ \\
\hline 1,1,1,2-Tetrachloroethane & $\mu \mathrm{g} / \mathrm{L}$ & U.S. & 70 & EPA HA & $<0.4$ & $<0.4$ & $<0.4$ & $<0.4$ & $<0.4$ & $<1.2$ & $<0.4$ & $<0.4$ \\
\hline 1,1,2,2-Tetrachloroethane & $\mu \mathrm{g} / \mathrm{L}$ & U.S. & 0.3 & EPA HA & $<0.4$ & $<0.4$ & $<0.4$ & $<0.4$ & $<0.4$ & $<1.2$ & $<0.4$ & $<0.4$ \\
\hline Tetrachloroethene & $\mu \mathrm{g} / \mathrm{L}$ & U.S. & 5 & EPA & $<0.4$ & $<0.4$ & $<0.4$ & $<0.4$ & $<0.4$ & $<1.2$ & $<0.4$ & $<0.4$ \\
\hline Tetrahydrofuran & $\mu \mathrm{g} / \mathrm{L}$ & U.S. & & & $<2$ & $<2$ & $<2$ & $<2$ & $<2$ & $<6$ & $<2$ & $<2$ \\
\hline \begin{tabular}{|l|} 
Toluene \\
\end{tabular} & $\mu \mathrm{g} / \mathrm{L}$ & U.S. & 1,000 & EPA & $<0.4$ & $<0.4$ & $<0.4$ & $<0.4$ & $<0.4$ & $<1.2$ & $<0.4$ & $<0.4$ \\
\hline 1,2,3-Trichlorobenzene & $\mu \mathrm{g} / \mathrm{L}$ & U.S. & & & $<0.4$ & $<0.4$ & $<0.4$ & $<0.4$ & $<0.4$ & $<1.2$ & $<0.4$ & $<0.4$ \\
\hline 1,2,4-Trichlorobenzene & $\mu \mathrm{g} / \mathrm{L}$ & U.S. & 70 & EPA & $<0.4$ & $<0.4$ & $<0.4$ & $<0.4$ & $<0.4$ & $<1.2$ & $<0.4$ & $<0.4$ \\
\hline 1,1,1-Trichloroethane & $\mu \mathrm{g} / \mathrm{L}$ & U.S. & 200 & EPA & $<0.4$ & $<0.4$ & $<0.4$ & $<0.4$ & $<0.4$ & $<1.2$ & $<0.4$ & $<0.4$ \\
\hline 1,1,2-Trichloroethane & $\mu \mathrm{g} / \mathrm{L}$ & U.S. & 5 & EPA & $<0.4$ & $<0.4$ & $<0.4$ & $<0.4$ & $<0.4$ & $<1.2$ & $<0.4$ & $<0.4$ \\
\hline Trichloroethene & $\mu \mathrm{g} / \mathrm{L}$ & U.S. & 5 & EPA & $<0.4$ & $<0.4$ & $<0.4$ & $<0.4$ & $<0.4$ & $<1.2$ & $<0.4$ & $<0.4$ \\
\hline Trichlorofluoromethane & $\mu \mathrm{g} / \mathrm{L}$ & U.S. & 2,000 & EPA HA & $<0.4$ & $<0.4$ & $<0.4$ & $<0.4$ & $<0.4$ & $<1.2$ & $<0.4$ & $<0.4$ \\
\hline 1,2,3-Trichloropropane & $\mu \mathrm{g} / \mathrm{L}$ & U.S. & 40 & EPA HA & $<0.4$ & $<0.4$ & $<0.4$ & $<0.4$ & $<0.4$ & $<1.2$ & $<0.4$ & $<0.4$ \\
\hline 1,2,4-Trimethylbenzene & $\mu \mathrm{g} / \mathrm{L}$ & U.S. & & & $<0.4$ & $<0.4$ & $<0.4$ & $<0.4$ & $<0.4$ & $<1.2$ & $<0.4$ & $<0.4$ \\
\hline $1,3,5$-Trimethylbenzene & $\mu \mathrm{g} / \mathrm{L}$ & U.S. & & & $<0.4$ & $<0.4$ & $<0.4$ & $<0.4$ & $<0.4$ & $<1.2$ & $<0.4$ & $<0.4$ \\
\hline Vinyl Acetate & $\mu \mathrm{g} / \mathrm{L}$ & U.S. & & & $<2$ & $<2$ & $<2$ & $<2$ & $<2$ & $<6$ & $<2$ & $<2$ \\
\hline Vinyl Chloride & $\mu \mathrm{g} / \mathrm{L}$ & U.S. & 2 & EPA & $<2$ & $<2$ & $<2$ & $<2$ & $<2$ & $<6$ & $<2$ & $<2$ \\
\hline m\&p-Xylene & $\mu \mathrm{g} / \mathrm{L}$ & U.S. & \begin{tabular}{|l|} 
Total Xylenes 10,000 \\
\end{tabular} & EPA & $<0.4$ & $<0.4$ & $<0.4$ & $<0.4$ & $<0.4$ & $<1.2$ & $<0.4$ & $<0.4$ \\
\hline 0 -Xylene & $\mu \mathrm{g} / \mathrm{L}$ & U.S. & \begin{tabular}{|l|} 
Total X ylenes 10,000 \\
\end{tabular} & EPA & $<0.4$ & $<0.4$ & $<0.4$ & $<0.4$ & $<0.4$ & $<1.2$ & $<0.4$ & $<0.4$ \\
\hline
\end{tabular}


Appendix 2. ISS SVO-ZV Potable Water Summary for Expeditions 21 through 25

\begin{tabular}{|c|c|c|c|c|c|c|c|c|c|c|c|c|}
\hline \multirow{3}{*}{\begin{tabular}{|l|} 
Mission \\
Sample Location \\
Sample Description \\
Sample Date \\
Analysis/Sample ID \\
\end{tabular}} & \multirow[b]{3}{*}{ Units } & \multirow[b]{3}{*}{$\begin{array}{c}\text { Test } \\
\text { Conducted } \\
\text { by }\end{array}$} & \multirow{3}{*}{$\begin{array}{c}\text { Potable W ater } \\
\text { Maximum } \\
\text { Contaminant } \\
\text { Level } \\
\text { (MCL) } \\
\end{array}$} & \multirow{3}{*}{$\begin{array}{c}\text { Maximum } \\
\text { Contaminant } \\
\text { Level } \\
\text { Source }\end{array}$} & \multicolumn{2}{|c|}{\begin{tabular}{|l|l|} 
Soyuz 19/Exp. 21 & ISS ULF3/Exp. 21 \\
\end{tabular}} & \multicolumn{2}{|c|}{ ISS 20A/Exp. 22} & \multirow{2}{*}{\begin{tabular}{|c|} 
Soyuz 20/Exp. 22 \\
SVO-ZV
\end{tabular}} & \multirow{2}{*}{\begin{tabular}{|c|} 
Soyuz 21/Exp. 23 \\
SVO-ZV
\end{tabular}} & \multicolumn{2}{|c|}{ ISS ULF4/Exp. 23} \\
\hline & & & & & SVO-ZV & SVO-ZV & SVO-ZV & SVO-ZV & & & SVO-ZV & SVO-ZV \\
\hline & & & & & $\begin{array}{c}\text { Potable W ater } \\
10 / 20 / 2009 \\
20091214003\end{array}$ & $\begin{array}{c}\text { Potable Water } \\
11 / 10 / 2009 \\
20091130010\end{array}$ & $\begin{array}{c}\text { Potable } W \text { ater } \\
1 / 6 / 2010 \\
20100222005\end{array}$ & \begin{tabular}{|c|} 
Potable W ater \\
$2 / 3 / 2010$ \\
20100222007
\end{tabular} & $\begin{array}{c}\text { Potable W ater } \\
3 / 3 / 2010 \\
20100326001\end{array}$ & $\begin{array}{c}\text { Potable Water } \\
3 / 31 / 2010 \\
20100603002\end{array}$ & $\begin{array}{c}\text { Potable Water } \\
4 / 26 / 2010 \\
20100527009\end{array}$ & $\begin{array}{c}\text { Potable Water } \\
5 / 18 / 2010 \\
20100527013\end{array}$ \\
\hline \multicolumn{13}{|c|}{ Volatile Organics - Non-Targets (Tentatively Identified Compounds ( $>\mid=80 \%$ match quality) } \\
\hline Acetaldehyde & $\mu \mathrm{g} / \mathrm{L}$ & U.S. & & & not found & not found & not found & not found & not found & not found & not found & not found \\
\hline Butyraldehyde (Butanal) & $\mu \mathrm{g} / \mathrm{L}$ & U.S. & & & not found & not found & not found & not found & not found & not found & not found & not found \\
\hline 1,8-Cineole & $\mu \mathrm{g} / \mathrm{L}$ & U.S. & & & not found & not found & not found & not found & not found & not found & not found & not found \\
\hline Cyclohexanone & $\mu \mathrm{g} / \mathrm{L}$ & U.S. & & & not found & not found & not found & not found & not found & not found & not found & not found \\
\hline Difluorodimethylsilane & $\mu \mathrm{g} / \mathrm{L}$ & U.S. & & & not found & not found & not found & not found & not found & not found & not found & not found \\
\hline Dimethoxymethane (Formal) & $\mu \mathrm{g} / \mathrm{L}$ & U.S. & & & not found & not found & not found & not found & not found & not found & not found & not found \\
\hline 2,5-Dimethylfuran & $\mu \mathrm{g} / \mathrm{L}$ & U.S. & & & not found & not found & not found & not found & not found & not found & not found & not found \\
\hline 2,6-Dimethyl-1,7-octadiene & $\mu \mathrm{g} / \mathrm{L}$ & U.S. & & & not found & not found & not found & not found & not found & not found & not found & not found \\
\hline Dimethyl sulfide (Thiobismethane) & $\mu \mathrm{g} / \mathrm{L}$ & U.S. & & & not found & not found & not found & not found & not found & not found & not found & not found \\
\hline p-Dioxane & $\mu \mathrm{g} / \mathrm{L}$ & U.S. & & & not found & not found & not found & not found & not found & not found & not found & not found \\
\hline 1,3-Dioxolane (Ethylene glycol formal) & $\mu \mathrm{g} / \mathrm{L}$ & U.S. & & & not found & not found & not found & not found & not found & not found & not found & not found \\
\hline Ethyl acetate & $\mu \mathrm{g} / \mathrm{L}$ & U.S. & & & not found & not found & not found & not found & not found & not found & not found & not found \\
\hline Fluorotrimethylsilane & $\mu \mathrm{g} / \mathrm{L}$ & U.S. & & & not found & not found & not found & not found & not found & not found & not found & not found \\
\hline 2-Heptanone & $\mu \mathrm{g} / \mathrm{L}$ & U.S. & & & not found & not found & not found & not found & not found & not found & not found & not found \\
\hline 3-Hexanone & $\mu \mathrm{g} / \mathrm{L}$ & U.S. & & & not found & not found & not found & not found & not found & not found & not found & not found \\
\hline 3-Hydroxy-3-methylbutyric acid & $\mu \mathrm{g} / \mathrm{L}$ & U.S. & & & not found & not found & not found & not found & not found & not found & not found & not found \\
\hline Isobutyronitrile & $\mu \mathrm{g} / \mathrm{L}$ & U.S. & & & not found & not found & not found & not found & not found & not found & not found & not found \\
\hline Isooctanol & $\mu \mathrm{g} / \mathrm{L}$ & U.S. & & & not found & not found & not found & not found & not found & not found & not found & not found \\
\hline L-Menthol & $\mu \mathrm{g} / \mathrm{L}$ & U.S. & & & not found & not found & not found & not found & not found & not found & not found & not found \\
\hline Menthone & $\mu \mathrm{g} / \mathrm{L}$ & U.S. & & & not found & not found & not found & not found & not found & not found & not found & not found \\
\hline Methyl acetate & $\mu \mathrm{g} / \mathrm{L}$ & U.S. & & & not found & not found & not found & not found & not found & not found & not found & not found \\
\hline cis-1-Methyl-4-(1-methylethenyl)-cyclohexane & $\mu \mathrm{g} / \mathrm{L}$ & U.S. & & & not found & not found & not found & not found & not found & not found & not found & not found \\
\hline 3-Methyl-2-pentanone & $\mu \mathrm{g} / \mathrm{L}$ & U.S. & & & not found & not found & not found & not found & not found & not found & not found & not found \\
\hline 2-Methyl-1-propene & $\mu \mathrm{g} / \mathrm{L}$ & U.S. & & & not found & not found & not found & not found & not found & not found & not found & not found \\
\hline \begin{tabular}{|l|} 
alpha-Methyl styrene \\
\end{tabular} & $\mu \mathrm{g} / \mathrm{L}$ & U.S. & & & not found & not found & not found & not found & not found & not found & not found & not found \\
\hline 2-Nonanone & $\mu \mathrm{g} / \mathrm{L}$ & U.S. & & & not found & not found & not found & not found & not found & not found & not found & not found \\
\hline 2-Octanone & $\mu \mathrm{g} / \mathrm{L}$ & U.S. & & & not found & not found & not found & not found & not found & not found & not found & not found \\
\hline 2-Pentanone & $\mu \mathrm{g} / \mathrm{L}$ & U.S. & & & not found & not found & not found & not found & not found & not found & not found & not found \\
\hline 1,1,2-Trichloro-1,2,2-trifluoroethane (Freon 113) & $\mu \mathrm{g} / \mathrm{L}$ & U.S. & & & not found & not found & not found & not found & not found & not found & not found & not found \\
\hline Trimethylsilanol & $\mu \mathrm{g} / \mathrm{L}$ & U.S. & & & not found & not found & not found & not found & not found & not found & not found & not found \\
\hline & & & & & & & & & & & & \\
\hline \multicolumn{13}{|l|}{\begin{tabular}{|l|} 
Extractable Organics \\
\end{tabular}} \\
\hline \begin{tabular}{|l|} 
Acetophenone \\
\end{tabular} & $\mu \mathrm{g} / \mathrm{L}$ & U.S. & & & $<16$ & $<8$ & $<8$ & $<8$ & $<8$ & $<32$ & $<16$ & $<16$ \\
\hline \begin{tabular}{|l|} 
Benzaldehyde \\
\end{tabular} & $\mu \mathrm{g} / \mathrm{L}$ & U.S. & & & $<8$ & $<4$ & $<4$ & $<4$ & $<4$ & $<16$ & $<8$ & $<8$ \\
\hline Benzoic acid & $\mu \mathrm{g} / \mathrm{L}$ & U.S. & & & $<24$ & $<12$ & $<12$ & $<12$ & $<12$ & $<48$ & $<24$ & $<24$ \\
\hline Benzothiazole & $\mu \mathrm{g} / \mathrm{L}$ & U.S. & & & $<8$ & $<4$ & $<4$ & $<4$ & $<4$ & $<16$ & $<8$ & $<8$ \\
\hline \begin{tabular}{|l|} 
Benzyl alcohol \\
\end{tabular} & $\mu \mathrm{g} / \mathrm{L}$ & U.S. & & & $<8$ & $<4$ & $<4$ & $<4$ & $<4$ & $<16$ & $<8$ & $<8$ \\
\hline Benzyl butyl phthlate & $\mu \mathrm{g} / \mathrm{L}$ & U.S. & 7,000 & EPA DWEL & $<8$ & $<4$ & $<4$ & $<4$ & $<4$ & $<16$ & $<8$ & $<8$ \\
\hline 2-Butoxyethanol & $\mu \mathrm{g} / \mathrm{L}$ & U.S. & & & $<16$ & $<8$ & $<8$ & $<8$ & $<8$ & $<32$ & $<16$ & $<16$ \\
\hline 2-(2-Butoxyethoxy)ethanol & $\mu \mathrm{g} / \mathrm{L}$ & U.S. & & & $<16$ & $<8$ & $<8$ & $<8$ & $<8$ & $<32$ & $<16$ & $<16$ \\
\hline 2-(2-Butoxyethoxy)ethyl acetate & $\mu \mathrm{g} / \mathrm{L}$ & U.S. & & & $<8$ & $<4$ & $<4$ & $<4$ & $<4$ & $<16$ & $<8$ & $<8$ \\
\hline n-Butylpalmitate & $\mu \mathrm{g} / \mathrm{L}$ & U.S. & & & $<16$ & $<8$ & $<8$ & $<8$ & $<8$ & $<32$ & $<16$ & $<16$ \\
\hline Butylated hydroxyanisole (BHA) & $\mu \mathrm{g} / \mathrm{L}$ & U.S. & & & $<8$ & $<4$ & $<4$ & $<4$ & $<4$ & $<16$ & $<8$ & $<8$ \\
\hline N-Butylbenzenesulfonamide & $\mu \mathrm{g} / \mathrm{L}$ & U.S. & & & $<8$ & $<4$ & $<4$ & $<4$ & $<4$ & $<16$ & $<8$ & $<8$ \\
\hline 3-tert-Butylphenol & $\mu \mathrm{g} / \mathrm{L}$ & U.S. & & & $<24$ & $<12$ & $<12$ & $<12$ & $<12$ & $\angle 48$ & $<24$ & $<24$ \\
\hline Caffeine & $\mu \mathrm{g} / \mathrm{L}$ & U.S. & & & $<8$ & $<4$ & $<4$ & $<4$ & $<4$ & $<16$ & $<8$ & $<8$ \\
\hline tris-2-Chloroethyl phosphate & $\mu \mathrm{g} / \mathrm{L}$ & U.S. & & & $<8$ & $<4$ & $<4$ & $<4$ & $<4$ & $<16$ & $<8$ & $<8$ \\
\hline
\end{tabular}

NA=Not analyzed; MI=Matrix interference

*MORD limit $1.5 \mathrm{mg} / \mathrm{L}$ (Russian method)
**limit does not include contribution from formate

\#TDS allowable limit after mineralization

SWEG - 1000 days (5-2006) 
Appendix 2. ISS SVO-ZV Potable Water Summary for Expeditions 21 through 25

\begin{tabular}{|c|c|c|c|c|c|c|c|c|c|c|c|c|}
\hline Mission & & & & & Soyuz 19/Exp. 21 & ISS ULF3/Exp. 21 & ISS 20A & /Exp. 22 & Soyuz 20/Exp. 22 & \begin{tabular}{|l|} 
Soyuz 21/Exp. 23 \\
\end{tabular} & ISS ULF4 & 4/Exp. 23 \\
\hline Sample Location & & & $\begin{array}{l}\text { Potable W ater } \\
\text { Maximum }\end{array}$ & Maximum & SVO-ZV & SVO-ZV & SVO-ZV & SVO-ZV & SVO-ZV & SVO-ZV & SVO-ZV & SVO-ZV \\
\hline $\begin{array}{l}\text { Sample Description } \\
\text { Sample Date } \\
\text { Analysis/Sample ID }\end{array}$ & Units & $\begin{array}{c}\text { Test } \\
\text { Conducted } \\
\text { by }\end{array}$ & $\begin{array}{c}\text { Contaminant } \\
\text { Level } \\
(\mathrm{MCL}) \\
\end{array}$ & $\begin{array}{c}\text { Contaminant } \\
\text { Level } \\
\text { Source } \\
\end{array}$ & $\begin{array}{l}\text { Potable W ater } \\
10 / 20 / 2009 \\
20091214003\end{array}$ & $\begin{array}{c}\text { Potable W ater } \\
11 / 10 / 2009 \\
20091130010 \\
\end{array}$ & $\begin{array}{c}\text { Potable } W \text { ater } \\
1 / 6 / 2010 \\
20100222005 \\
\end{array}$ & \begin{tabular}{|c|} 
Potable W ater \\
$2 / 3 / 2010$ \\
20100222007 \\
\end{tabular} & $\begin{array}{c}\text { Potable W ater } \\
3 / 3 / 2010 \\
20100326001\end{array}$ & $\begin{array}{c}\text { Potable W ater } \\
3 / 31 / 2010 \\
20100603002 \\
\end{array}$ & \begin{tabular}{|c|} 
Potable $W$ ater \\
$4 / 26 / 2010$ \\
20100527009 \\
\end{tabular} & $\begin{array}{c}\text { Potable W ater } \\
5 / 18 / 2010 \\
20100527013 \\
\end{array}$ \\
\hline \begin{tabular}{|l|} 
Cholesterol \\
\end{tabular} & $\mu \mathrm{g} / \mathrm{L}$ & U.S. & & & $<64$ & $<32$ & $\begin{array}{c}<32 \\
<3\end{array}$ & $<32$ & $<32$ & $<128$ & \begin{tabular}{|c|}
20004 \\
$<64$ \\
\end{tabular} & $<64$ \\
\hline 0-Cresol (2-Methylphenol) & $\mu \mathrm{g} / \mathrm{L}$ & U.S. & & & $<8$ & $<4$ & $<4$ & $<4$ & $<4$ & $<16$ & $<8$ & $<8$ \\
\hline Cyclododecane & $\mu \mathrm{g} / \mathrm{L}$ & U.S. & & & $<8$ & $<4$ & $<4$ & $<4$ & $<4$ & $<16$ & $<8$ & $<8$ \\
\hline Decamethylcyclopentasiloxane & $\mu \mathrm{g} / \mathrm{L}$ & U.S. & & & $<8$ & $<4$ & $<4$ & $<4$ & $<4$ & $<16$ & $<8$ & $<8$ \\
\hline \begin{tabular}{|l|} 
Decanoic acid \\
\end{tabular} & $\mu \mathrm{g} / \mathrm{L}$ & U.S. & & & $<16$ & $<8$ & $<8$ & $<8$ & $<8$ & $<32$ & $<16$ & $<16$ \\
\hline 2,6-Di-t-butyl-1,4-benzoquinone & $\mu \mathrm{g} / \mathrm{L}$ & U.S. & & & $<8$ & $<4$ & $<4$ & $<4$ & $<4$ & $<16$ & $<8$ & $<8$ \\
\hline 2,4-Di-t-butylphenol & $\mu \mathrm{g} / \mathrm{L}$ & U.S. & & & $<8$ & $<4$ & $<4$ & $<4$ & $<4$ & $<16$ & $<8$ & $<8$ \\
\hline 1,4 Diacetylbenzene & $\mu \mathrm{g} / \mathrm{L}$ & U.S. & & & $<8$ & $<4$ & $<4$ & $<4$ & $<4$ & $<16$ & $<8$ & $<8$ \\
\hline N,N-Dibutylformamide & $\mu \mathrm{g} / \mathrm{L}$ & U.S. & & & $<8$ & $<4$ & $<4$ & $<4$ & $<4$ & $<16$ & $<8$ & $<8$ \\
\hline \begin{tabular}{|l|} 
Dibutyl phthalate \\
\end{tabular} & $\mu \mathrm{g} / \mathrm{L}$ & U.S. & $40,000 / 4,000$ & SWEG/EPA DWEL & $<8$ & $<4$ & $<4$ & $<4$ & $<4$ & $<16$ & $<8$ & $<8$ \\
\hline \begin{tabular}{|l|} 
Dibutylamine \\
\end{tabular} & $\mu \mathrm{g} / \mathrm{L}$ & U.S. & \begin{tabular}{|l|} 
Dialkylamines 300 \\
\end{tabular} & SWEG & $<8$ & $<4$ & $<4$ & $<4$ & $<4$ & $<16$ & $<8$ & $<8$ \\
\hline \begin{tabular}{|l|}
$\mathrm{N}, \mathrm{N}$-Diethyl-m-toluamide \\
\end{tabular} & $\mu \mathrm{g} / \mathrm{L}$ & U.S. & & & $<8$ & $<4$ & $<4$ & $<4$ & $<4$ & $<16$ & $<8$ & $<8$ \\
\hline Diethylphthalate & $\mu \mathrm{g} / \mathrm{L}$ & U.S. & 30,000 & EPA DWEL & $<8$ & $<4$ & $<4$ & $<4$ & $<4$ & $<16$ & $<8$ & $<8$ \\
\hline Diethylene glycol monoethyl ether & $\mu \mathrm{g} / \mathrm{L}$ & U.S. & & & $<8$ & $<4$ & $<4$ & $<4$ & $<4$ & $<16$ & $<8$ & $<8$ \\
\hline N,N-Diethylformamide & $\mu \mathrm{g} / \mathrm{L}$ & U.S. & & & $<24$ & $<12$ & $<12$ & $<12$ & $<12$ & $<48$ & $<24$ & $<24$ \\
\hline \begin{tabular}{|l|} 
Diiodomethane (Methyl iodide) \\
\end{tabular} & $\mu \mathrm{g} / \mathrm{L}$ & U.S. & & & $<8$ & $<4$ & $<4$ & $<4$ & $<4$ & $<16$ & $<8$ & $<8$ \\
\hline \begin{tabular}{|l|} 
Diisopropyl adipate \\
\end{tabular} & $\mu \mathrm{g} / \mathrm{L}$ & U.S. & & & $<8$ & $<4$ & $<4$ & $<4$ & $<4$ & $<16$ & $<8$ & $<8$ \\
\hline \begin{tabular}{|l} 
Dimethyl phthalate \\
\end{tabular} & $\mu \mathrm{g} / \mathrm{L}$ & U.S. & & & $<8$ & $<4$ & $<4$ & $<4$ & $<4$ & $<16$ & $<8$ & $<8$ \\
\hline N,N-Dimethyl acetamide & $\mu \mathrm{g} / \mathrm{L}$ & U.S. & & & $<8$ & $<4$ & $<4$ & $<4$ & $<4$ & $<16$ & $<8$ & $<8$ \\
\hline N,N-Dimethylbenzylamine & $\mu \mathrm{g} / \mathrm{L}$ & U.S. & \begin{tabular}{|l|} 
Dialkylamines 300 \\
\end{tabular} & SWEG & $<8$ & $<4$ & $<4$ & $<4$ & $<4$ & $<16$ & $<8$ & $<8$ \\
\hline N,N-Dimethylformamide & $\mu \mathrm{g} / \mathrm{L}$ & U.S. & & & $<16$ & $<8$ & $<8$ & $<8$ & $<8$ & $<32$ & $<16$ & $<16$ \\
\hline \begin{tabular}{|l|} 
Dipropylene glycol methyl ether \\
\end{tabular} & $\mu \mathrm{g} / \mathrm{L}$ & U.S. & & & $<8$ & $<4$ & $<4$ & $<4$ & $<4$ & $<16$ & $<8$ & $<8$ \\
\hline \begin{tabular}{|l|} 
Dodecamethylcyclohexasiloxane \\
\end{tabular} & $\mu \mathrm{g} / \mathrm{L}$ & U.S. & & & $<8$ & $<4$ & $<4$ & $<4$ & $<4$ & $<16$ & $<8$ & $<8$ \\
\hline 2-Ethoxyethanol & $\mu \mathrm{g} / \mathrm{L}$ & U.S. & & & $<8$ & $<4$ & $<4$ & $<4$ & $<4$ & $<16$ & $<8$ & $<8$ \\
\hline 2-Ethyl-1-hexanol & $\mu \mathrm{g} / \mathrm{L}$ & U.S. & & & $<8$ & $<4$ & $<4$ & $<4$ & $<4$ & $<16$ & $<8$ & $<8$ \\
\hline 2-Ethylhexanoic acid & $\mu \mathrm{g} / \mathrm{L}$ & U.S. & & & $<8$ & $<4$ & $<4$ & $<4$ & $<4$ & $<16$ & $<8$ & $<8$ \\
\hline bis-2-Ethylhexyl adipate & $\mu \mathrm{g} / \mathrm{L}$ & U.S. & 400 & EPA & $<8$ & $<4$ & $<4$ & $<4$ & $<4$ & $<16$ & $<8$ & $<8$ \\
\hline bis-2-Ethylhexyl phthalate (Dioctyl phthlate) & $\mu \mathrm{g} / \mathrm{L}$ & U.S. & $20,000 / 6$ & SWEG/EPA & $<8$ & $<4$ & $<4$ & $<4$ & $<4$ & $<16$ & $<8$ & $<8$ \\
\hline 4-Ethylmorpholine & $\mu \mathrm{g} / \mathrm{L}$ & U.S. & & & $<8$ & $<4$ & $<4$ & $<4$ & $<4$ & $\angle 16$ & $<8$ & $<8$ \\
\hline 1-Formylpiperidine & $\mu \mathrm{g} / \mathrm{L}$ & U.S. & & & $<8$ & $<4$ & $<4$ & $<4$ & $<4$ & $<16$ & $<8$ & $<8$ \\
\hline Heptanoic acid & $\mu \mathrm{g} / \mathrm{L}$ & U.S. & & & $<8$ & $<4$ & $<4$ & $<4$ & $<4$ & $<16$ & $<8$ & $<8$ \\
\hline 2-Heptanone & $\mu \mathrm{g} / \mathrm{L}$ & U.S. & & & $<8$ & $<4$ & $<4$ & $<4$ & $<4$ & $<16$ & $<8$ & $<8$ \\
\hline gamma-Hexalactone & $\mu \mathrm{g} / \mathrm{L}$ & U.S. & & & $<8$ & $<4$ & $<4$ & $<4$ & $<4$ & $\angle 16$ & $<8$ & $<8$ \\
\hline Hexanoic acid & $\mu \mathrm{g} / \mathrm{L}$ & U.S. & & & $<16$ & $<8$ & $<8$ & $<8$ & $<8$ & $<32$ & $<16$ & $<16$ \\
\hline 2-Hexanol & $\mu \mathrm{g} / \mathrm{L}$ & U.S. & & & $<8$ & $<4$ & $<4$ & $<4$ & $<4$ & $<16$ & $<8$ & $<8$ \\
\hline 2-Hydroxybenzothiazole & $\mu \mathrm{g} / \mathrm{L}$ & U.S. & & & $<8$ & $<4$ & $<4$ & $<4$ & $<4$ & $<16$ & $<8$ & $<8$ \\
\hline Ibuprofen & $\mu \mathrm{g} / \mathrm{L}$ & U.S. & & & $<8$ & $<4$ & $<4$ & $<4$ & $<4$ & $<16$ & $<8$ & $<8$ \\
\hline Iodoform & $\mu \mathrm{g} / \mathrm{L}$ & U.S. & & & $<8$ & $<4$ & $<4$ & $<4$ & $<4$ & $<16$ & $<8$ & $<8$ \\
\hline Isophorone & $\mu \mathrm{g} / \mathrm{L}$ & U.S. & 100 & EPA HA & $<8$ & $<4$ & $<4$ & $<4$ & $<4$ & $<16$ & $<8$ & $<8$ \\
\hline 4-Isopropylphenol & $\mu \mathrm{g} / \mathrm{L}$ & U.S. & & & $<8$ & $<4$ & $<4$ & $<4$ & $<4$ & $<16$ & $<8$ & $<8$ \\
\hline \begin{tabular}{|l} 
Lauramide \\
\end{tabular} & $\mu \mathrm{g} / \mathrm{L}$ & U.S. & & & $<8$ & $<4$ & $<4$ & $<4$ & $<4$ & $<16$ & $<8$ & $<8$ \\
\hline Lauric acid (Dodecanoic acid) & $\mu \mathrm{g} / \mathrm{L}$ & U.S. & & & $<240$ & $<120$ & $<120$ & $<120$ & $<120$ & $<480$ & $<240$ & $<240$ \\
\hline p-Menth-1-en-8-ol (alpha-Terpineol) & $\mu \mathrm{g} / \mathrm{L}$ & U.S. & & & $<8$ & $<4$ & $<4$ & $<4$ & $<4$ & $<16$ & $<8$ & $<8$ \\
\hline 2-Mercaptobenzothiazole & $\mu \mathrm{g} / \mathrm{L}$ & U.S. & 30,000 & SWEG & $<80$ & $\angle 40$ & $\angle 40$ & $\angle 40$ & $\angle 40$ & $<160$ & $<80$ & $<80$ \\
\hline 2-Methyl-2,4-pentanediol & $\mu \mathrm{g} / \mathrm{L}$ & U.S. & & & $<8$ & $<4$ & $<4$ & $<4$ & $<4$ & $<16$ & $<8$ & $<8$ \\
\hline \begin{tabular}{|l|} 
1-Methyl-2-pyrrolidinone \\
\end{tabular} & $\mu \mathrm{g} / \mathrm{L}$ & U.S. & & & $<8$ & $<4$ & $<4$ & $<4$ & $<4$ & $<16$ & $<8$ & $<8$ \\
\hline Methyl-4-hydroxybenzoate & $\mu \mathrm{g} / \mathrm{L}$ & U.S. & & & $<8$ & $<4$ & $<4$ & $<4$ & $<4$ & $<16$ & $<8$ & $<8$ \\
\hline Methyl sulfone & $\mu \mathrm{g} / \mathrm{L}$ & U.S. & & & $<8$ & $<4$ & $<4$ & $<4$ & $<4$ & $<16$ & $<8$ & $<8$ \\
\hline
\end{tabular}


Appendix 2. ISS SVO-ZV Potable Water Summary for Expeditions 21 through 25

\begin{tabular}{|c|c|c|c|c|c|c|c|c|c|c|c|c|}
\hline Mission & \multirow[b]{3}{*}{ Units } & \multirow[b]{3}{*}{\begin{tabular}{|c|} 
Test \\
Conducted \\
by
\end{tabular}} & \multirow{3}{*}{$\begin{array}{c}\text { Potable W ater } \\
\text { Maximum } \\
\text { Contaminant } \\
\text { Level } \\
\text { (MCL) } \\
\end{array}$} & \multirow{3}{*}{$\begin{array}{c}\text { Maximum } \\
\text { Contaminant } \\
\text { Level } \\
\text { Source } \\
\end{array}$} & \multicolumn{2}{|c|}{\begin{tabular}{|l|l|} 
Soyuz 19/Exp. 21 & ISS ULF3/Exp. 21 \\
\end{tabular}} & \multicolumn{2}{|c|}{ ISS 20A/Exp. 22} & \multirow{2}{*}{$\begin{array}{c}\text { Soyuz 20/Exp. 22 } \\
\text { SVO-ZV }\end{array}$} & \multirow{2}{*}{\begin{tabular}{|c|} 
Soyuz 21/Exp. 23 \\
SVO-ZV
\end{tabular}} & \multicolumn{2}{|c|}{ ISS ULF4/Exp. 23} \\
\hline Sample Location & & & & & SVO-ZV & SVO-ZV & SVO-ZV & SVO-ZV & & & SVO-ZV & SVO-ZV \\
\hline $\begin{array}{l}\text { Sample Description } \\
\text { Sample Date } \\
\text { Analysis/Sample ID }\end{array}$ & & & & & $\begin{array}{l}\text { Potable W ater } \\
10 / 20 / 2009 \\
20091214003\end{array}$ & $\begin{array}{c}\text { Potable W ater } \\
11 / 10 / 2009 \\
20091130010 \\
\end{array}$ & $\begin{array}{c}\text { Potable } W \text { ater } \\
1 / 6 / 2010 \\
20100222005 \\
\end{array}$ & \begin{tabular}{|c|} 
Potable W ater \\
$2 / 3 / 2010$ \\
20100222007 \\
\end{tabular} & $\begin{array}{c}\text { Potable W ater } \\
3 / 3 / 2010 \\
20100326001\end{array}$ & $\begin{array}{c}\text { Potable W ater } \\
3 / 31 / 2010 \\
20100603002\end{array}$ & \begin{tabular}{|c|} 
Potable W ater \\
$4 / 26 / 2010$ \\
20100527009
\end{tabular} & $\begin{array}{c}\text { Potable } W \text { ater } \\
5 / 18 / 2010 \\
20100527013\end{array}$ \\
\hline 2-Methyl butyric acid & $\mu \mathrm{g} / \mathrm{L}$ & \begin{tabular}{|l|} 
U.S. \\
\end{tabular} & & & $<24$ & $<12$ & $<12$ & \begin{tabular}{|c|}
$<12$ \\
\end{tabular} & $<12$ & $<48$ & \begin{tabular}{|l|}
2024 \\
$<24$ \\
\end{tabular} & $<24$ \\
\hline 2-Methylthiobenzothiazole & $\mu \mathrm{g} / \mathrm{L}$ & U.S. & & & $<8$ & $<4$ & $<4$ & $<4$ & $<4$ & $<16$ & $<8$ & $<8$ \\
\hline Monomethyl phthalate & $\mu \mathrm{g} / \mathrm{L}$ & U.S. & & & $<8$ & $<4$ & $<4$ & $<4$ & $<4$ & $<16$ & $<8$ & $<8$ \\
\hline Myristic acid & $\mu \mathrm{g} / \mathrm{L}$ & U.S. & & & $<48$ & $<24$ & $<24$ & $<24$ & $<24$ & $<96$ & $<48$ & $<48$ \\
\hline (+)-Neomenthol & $\mu \mathrm{g} / \mathrm{L}$ & U.S. & & & $<8$ & $<4$ & $<4$ & $<4$ & $<4$ & $<16$ & $<8$ & $<8$ \\
\hline Nicotine & $\mu \mathrm{g} / \mathrm{L}$ & U.S. & & & $<8$ & $<4$ & $<4$ & $<4$ & $<4$ & $<16$ & $<8$ & $<8$ \\
\hline Nonadecane & $\mu \mathrm{g} / \mathrm{L}$ & U.S. & & & $<8$ & $<4$ & $<4$ & $<4$ & $<4$ & $<16$ & $<8$ & $<8$ \\
\hline \begin{tabular}{|l} 
Nonanoic acid \\
\end{tabular} & $\mu \mathrm{g} / \mathrm{L}$ & U.S. & & & $<24$ & $<12$ & $<12$ & $<12$ & $<12$ & $<48$ & $<24$ & $<24$ \\
\hline 1-Octadecanol & $\mu \mathrm{g} / \mathrm{L}$ & U.S. & & & $<24$ & $<12$ & $<12$ & $<12$ & $<12$ & $\angle 48$ & $<24$ & $<24$ \\
\hline Octamethylcyclotetrasiloxane & $\mu \mathrm{g} / \mathrm{L}$ & U.S. & & & $<8$ & $<4$ & $<4$ & $<4$ & $<4$ & $<16$ & $<8$ & $<8$ \\
\hline Octanoic acid & $\mu \mathrm{g} / \mathrm{L}$ & U.S. & & & $<16$ & $<8$ & $<8$ & $<8$ & $<8$ & $<32$ & $<16$ & $<16$ \\
\hline 4-tert-Octylphenol & $\mu \mathrm{g} / \mathrm{L}$ & U.S. & & & $<8$ & $<4$ & $<4$ & $<4$ & $<4$ & $<16$ & $<8$ & $<8$ \\
\hline \begin{tabular}{|l} 
Oleic acid \\
\end{tabular} & $\mu \mathrm{g} / \mathrm{L}$ & U.S. & & & $<80$ & $<40$ & $<40$ & $<40$ & $<40$ & $<160$ & $<80$ & $<80$ \\
\hline \begin{tabular}{|l|} 
Oxindole \\
\end{tabular} & $\mu \mathrm{g} / \mathrm{L}$ & U.S. & & & $<8$ & $<4$ & $<4$ & $<4$ & $<4$ & $<16$ & $<8$ & $<8$ \\
\hline Palmitic acid & $\mu \mathrm{g} / \mathrm{L}$ & U.S. & & & $<240$ & $<120$ & $<120$ & $<120$ & $<120$ & $\angle 480$ & $<240$ & $<240$ \\
\hline Palmitoleic acid & $\mu \mathrm{g} / \mathrm{L}$ & U.S. & & & $\mathrm{NA}$ & $\mathrm{NA}$ & $\mathrm{NA}$ & $\mathrm{NA}$ & $\mathrm{NA}$ & $\mathrm{NA}$ & $\mathrm{NA}$ & $\mathrm{NA}$ \\
\hline \begin{tabular}{|l|} 
Pentacosane \\
\end{tabular} & $\mu \mathrm{g} / \mathrm{L}$ & U.S. & & & $<8$ & $<4$ & $<4$ & $<4$ & $<4$ & $<16$ & $<8$ & $<8$ \\
\hline sec-Phenethyl alcohol & $\mu \mathrm{g} / \mathrm{L}$ & U.S. & & & $<8$ & $<4$ & $<4$ & $<4$ & $<4$ & $<16$ & $<8$ & $<8$ \\
\hline Phenol & $\mu \mathrm{g} / \mathrm{L}$ & U.S. & $1,000 / 4,000$ & MORD/SWEG & $<8$ & $<4$ & $<4$ & $<4$ & $<4$ & $<16$ & $<8$ & $<8$ \\
\hline 2-Phenoxyethanol & $\mu \mathrm{g} / \mathrm{L}$ & U.S. & & & $<8$ & $<4$ & $<4$ & $<4$ & $<4$ & $<16$ & $<8$ & $<8$ \\
\hline N-Phenyl-2-naphthylamine & $\mu \mathrm{g} / \mathrm{L}$ & U.S. & 260,000 & SWEG & $<8$ & $<4$ & $<4$ & $<4$ & $<4$ & $<16$ & $<8$ & $<8$ \\
\hline 2-Phenyl-2-propanol & $\mu \mathrm{g} / \mathrm{L}$ & U.S. & & & $<8$ & $<4$ & $<4$ & $<4$ & $<4$ & $<16$ & $<8$ & $<8$ \\
\hline 2-Phenylacetic acid & $\mu \mathrm{g} / \mathrm{L}$ & U.S. & & & $<32$ & $<16$ & $<16$ & $<16$ & $<16$ & $<64$ & $<32$ & $<32$ \\
\hline \begin{tabular}{|l|} 
Phenethyl alcohol \\
\end{tabular} & $\mu \mathrm{g} / \mathrm{L}$ & U.S. & & & $<8$ & $<4$ & $<4$ & $<4$ & $<4$ & $<16$ & $<8$ & $<8$ \\
\hline 2-Phenylphenol & $\mu \mathrm{g} / \mathrm{L}$ & U.S. & & & $<8$ & $<4$ & $<4$ & $<4$ & $<4$ & $<16$ & $<8$ & $<8$ \\
\hline Salicyclic Acid & $\mu \mathrm{g} / \mathrm{L}$ & U.S. & & & $<64$ & $<32$ & $<32$ & $<32$ & $<32$ & $<128$ & $<64$ & $<64$ \\
\hline trans-Squalene & $\mu \mathrm{g} / \mathrm{L}$ & U.S. & & & $<16$ & $<8$ & $<8$ & $<8$ & $<8$ & $<32$ & $<16$ & $<16$ \\
\hline \begin{tabular}{|l|} 
Stearic acid \\
\end{tabular} & $\mu \mathrm{g} / \mathrm{L}$ & U.S. & & & $\mathrm{NA}$ & $\mathrm{NA}$ & $\mathrm{NA}$ & NA & $\mathrm{NA}$ & $\mathrm{NA}$ & $\mathrm{NA}$ & $\mathrm{NA}$ \\
\hline 1-Tetradecanol & $\mu \mathrm{g} / \mathrm{L}$ & U.S. & & & $<8$ & $<4$ & $<4$ & $<4$ & $<4$ & $<16$ & $<8$ & $<8$ \\
\hline Tetramethylsuccinonitrile & $\mu \mathrm{g} / \mathrm{L}$ & U.S. & & & $<8$ & $<4$ & $<4$ & $<4$ & $<4$ & $<16$ & $<8$ & $<8$ \\
\hline Tetramethyl thiourea & $\mu \mathrm{g} / \mathrm{L}$ & U.S. & & & $<8$ & $<4$ & $<4$ & $<4$ & $<4$ & $<16$ & $<8$ & $<8$ \\
\hline Tetramethylurea & $\mu \mathrm{g} / \mathrm{L}$ & U.S. & & & $<8$ & $<4$ & $<4$ & $<4$ & $<4$ & $<16$ & $<8$ & $<8$ \\
\hline Thymol & $\mu \mathrm{g} / \mathrm{L}$ & U.S. & & & $<8$ & $<4$ & $<4$ & $<4$ & $<4$ & $<16$ & $<8$ & $<8$ \\
\hline 1,3,5-Triallyl-1,3,5-triazine-2,4,6(1H,3H,5H)-trione & $\mu \mathrm{g} / \mathrm{L}$ & U.S. & & & $<8$ & $<4$ & $<4$ & $<4$ & $<4$ & $<16$ & $<8$ & $<8$ \\
\hline Tributylamine & $\mu \mathrm{g} / \mathrm{L}$ & U.S. & Trialkylamines 400 & SWEG & $<8$ & $<4$ & $<4$ & $<4$ & $<4$ & $<16$ & $<8$ & $<8$ \\
\hline Tributyl phosphate & $\mu \mathrm{g} / \mathrm{L}$ & U.S. & & & $<8$ & $<4$ & $<4$ & $<4$ & $<4$ & $<16$ & $<8$ & $<8$ \\
\hline \begin{tabular}{|l|} 
Triethyl phosphate \\
\end{tabular} & $\mu \mathrm{g} / \mathrm{L}$ & U.S. & & & $<16$ & $<8$ & $<8$ & $<8$ & $<8$ & $<32$ & $<16$ & $<16$ \\
\hline 2,2,4-Trimethyl-1,3-pentanediol diisobutyrate & $\mu \mathrm{g} / \mathrm{L}$ & U.S. & & & $<16$ & $<8$ & $<8$ & $<8$ & $<8$ & $<32$ & $<16$ & $<16$ \\
\hline Tripropylene glycol monomethyl ether & $\mu \mathrm{g} / \mathrm{L}$ & U.S. & & & $<8$ & $<4$ & $<4$ & $<4$ & $<4$ & $<16$ & $<8$ & $<8$ \\
\hline Undecanoic acid & $\mu \mathrm{g} / \mathrm{L}$ & U.S. & & & $\angle 48$ & $<24$ & $<24$ & $<24$ & $<24$ & $<96$ & $\angle 48$ & $<48$ \\
\hline 2-Undecanone & $\mu \mathrm{g} / \mathrm{L}$ & U.S. & & & $<8$ & $<4$ & $<4$ & $<4$ & $<4$ & $<16$ & $<8$ & $<8$ \\
\hline Valeric acid (Pentanoic acid) & $\mu \mathrm{g} / \mathrm{L}$ & U.S. & & & $<48$ & $<24$ & $<24$ & $<24$ & $<24$ & $<96$ & $<48$ & $<48$ \\
\hline $\begin{array}{l}\text { Vanillin } \\
\end{array}$ & $\mu \mathrm{g} / \mathrm{L}$ & U.S. & & & $<16$ & $<8$ & $<8$ & $<8$ & $<8$ & $<32$ & $<16$ & $<16$ \\
\hline \multicolumn{13}{|l|}{\begin{tabular}{|l|} 
Alcohols (DAI/GC/M S) \\
\end{tabular}} \\
\hline 1-Butanol & $\mu \mathrm{g} / \mathrm{L}$ & U.S. & & & $<100$ & $<100$ & $<100$ & $<100$ & $<100$ & $<100$ & $<100$ & $<100$ \\
\hline 2-Butanol & $\mu \mathrm{g} / \mathrm{L}$ & U.S. & & & $<100$ & $<100$ & $<100$ & $<100$ & $<100$ & $<100$ & $<100$ & $<100$ \\
\hline Ethanol & $\mu \mathrm{g} / \mathrm{L}$ & U.S. & & & $<100$ & $<100$ & $<100$ & $<100$ & $<100$ & $<100$ & $<100$ & $<100$ \\
\hline
\end{tabular}




\section{Appendix 2. ISS SVO-ZV Potable Water Summary for Expeditions 21 through 25}

\begin{tabular}{|c|c|c|c|c|c|c|c|c|c|c|c|c|}
\hline Mission & & & & & Soyuz 19/Exp. 21 & SS ULF3/Exp. 21 & ISS 20A & Exp. 22 & Soyuz 20/Exp. 22 & Soyuz 21/Exp. 23 & ISS ULF4 & 4/Exp. 23 \\
\hline Sample Location & & & $\begin{array}{l}\text { Potable W ater } \\
\text { Maximum }\end{array}$ & Maximum & SVO-ZV & SVO-ZV & SVO-ZV & SVO-ZV & SVO-ZV & SVO-ZV & SVO-ZV & SVO-ZV \\
\hline $\begin{array}{l}\text { Sample Description } \\
\text { Sample Date } \\
\text { Analysis/Sample ID }\end{array}$ & Units & $\begin{array}{c}\text { Test } \\
\text { Conducted } \\
\text { by }\end{array}$ & $\begin{array}{c}\text { Contaminant } \\
\text { Level } \\
\text { (MCL) }\end{array}$ & $\begin{array}{c}\text { Contaminant } \\
\text { Level } \\
\text { Source } \\
\end{array}$ & $\begin{array}{c}\text { Potable W ater } \\
10 / 20 / 2009 \\
20091214003\end{array}$ & $\begin{array}{c}\text { Potable Water } \\
11 / 10 / 2009 \\
20091130010 \\
\end{array}$ & $\begin{array}{c}\text { Potable } W \text { ater } \\
1 / 6 / 2010 \\
20100222005\end{array}$ & \begin{tabular}{|c|} 
Potable W ater \\
$2 / 3 / 2010$ \\
20100222007 \\
\end{tabular} & $\begin{array}{l}\text { Potable W ater } \\
3 / 3 / 2010 \\
20100326001\end{array}$ & $\begin{array}{c}\text { Potable Water } \\
3 / 31 / 2010 \\
20100603002\end{array}$ & \begin{tabular}{|c|} 
Potable W ater \\
$4 / 26 / 2010$ \\
20100527009 \\
\end{tabular} & \begin{tabular}{|c} 
Potable W ater \\
$5 / 18 / 2010$ \\
20100527013
\end{tabular} \\
\hline Methanol & $\mu \mathrm{g} / \mathrm{L}$ & $\begin{array}{l}\text { U.S. } \\
\end{array}$ & & & $<100$ & $<100$ & $<100$ & $<100$ & $<100$ & $<100$ & \begin{tabular}{|l|}
$<100$ \\
\end{tabular} & $<100$ \\
\hline 2-Methyl-1-butanol & $\mu \mathrm{g} / \mathrm{L}$ & U.S. & & & $<100$ & $<100$ & $<100$ & $<100$ & $<100$ & $<100$ & $<100$ & $<100$ \\
\hline 2-Methyl-2-butanol & $\mu \mathrm{g} / \mathrm{L}$ & U.S. & & & $<100$ & $<100$ & $<100$ & $<100$ & $<100$ & $<100$ & $<100$ & $<100$ \\
\hline 3-Methyl-1-butanol (Isopentanol) & $\mu \mathrm{g} / \mathrm{L}$ & U.S. & & & $<100$ & $<100$ & $<100$ & $<100$ & $<100$ & $<100$ & $<100$ & $<100$ \\
\hline 2-Methyl-1-propanol & $\mu \mathrm{g} / \mathrm{L}$ & U.S. & & & $<100$ & $<100$ & $<100$ & $<100$ & $<100$ & $<100$ & $<100$ & $<100$ \\
\hline 2-Methyl-2-propanol & $\mu \mathrm{g} / \mathrm{L}$ & U.S. & & & $<100$ & $<100$ & $<100$ & $<100$ & $<100$ & $<100$ & $<100$ & $<100$ \\
\hline 1-Pentanol (Amyl alcohol) & $\mu \mathrm{g} / \mathrm{L}$ & U.S. & & & $<100$ & $<100$ & $<100$ & $<100$ & $<100$ & $<100$ & $<100$ & $<100$ \\
\hline 2-Pentanol (sec-Amyl alcohol) & $\mu \mathrm{g} / \mathrm{L}$ & U.S. & & & $<100$ & $<100$ & $<100$ & $<100$ & $<100$ & $<100$ & $<100$ & $<100$ \\
\hline 3-Pentanol & $\mu \mathrm{g} / \mathrm{L}$ & U.S. & & & $<100$ & $<100$ & $<100$ & $<100$ & $<100$ & $<100$ & $<100$ & $<100$ \\
\hline 1-Propanol & $\mu \mathrm{g} / \mathrm{L}$ & U.S. & & & $<100$ & $<100$ & $<100$ & $<100$ & $<100$ & $<100$ & $<100$ & $<100$ \\
\hline 2-Propanol (Isopropanol) & $\mu \mathrm{g} / \mathrm{L}$ & U.S. & & & $<100$ & $<100$ & $<100$ & $<100$ & $<100$ & $<100$ & $<100$ & $<100$ \\
\hline & & & & & & & & & & & & \\
\hline Glycols (DAI/GC/MS) & & & & & & & & & & & & \\
\hline 1,2-Ethanediol (Ethylene glycol) & $\mu \mathrm{g} / \mathrm{L}$ & U.S. & $12000 / 14000$ & MORD/EPA HA & $<1000$ & $<1000$ & $<1000$ & $<1000$ & $<1000$ & $<1000$ & $<1000$ & $<1000$ \\
\hline 1,2-Propanediol (Propylene glycol) & $\mu \mathrm{g} / \mathrm{L}$ & U.S. & & & $<500$ & $<500$ & $<500$ & $<500$ & $<500$ & $<500$ & $<500$ & $<500$ \\
\hline & & & & & & & & & & & & \\
\hline Glycerol (LC/M S/MS) & & & & & & & & & & & & \\
\hline Glycerol (1,2,3-Propanetriol) & $\mu \mathrm{g} / \mathrm{L}$ & U.S. & & & $\mathrm{NA}$ & $\mathrm{NA}$ & $\mathrm{NA}$ & $\mathrm{NA}$ & NA & $\mathrm{NA}$ & $\mathrm{NA}$ & $\mathrm{NA}$ \\
\hline Silanols (LC/MS/MS) (R\&D Meth & ble stan & dard not avai & lable) & & & & & & & & & \\
\hline Dimethylsilanediol (DMSD) & $\mu \mathrm{g} / \mathrm{L}$ & U.S. & & & $\mathrm{NA}$ & $\mathrm{NA}$ & $\mathrm{NA}$ & $\mathrm{NA}$ & $\mathrm{NA}$ & $\mathrm{NA}$ & $\mathrm{NA}$ & $\mathrm{NA}$ \\
\hline Carboxylates (CE) & & & & & & & & & & & & \\
\hline & & & & & & & & & & & & \\
\hline Acetate & $\mu \mathrm{g} / \mathrm{L}$ & U.S. & & & $<125$ & $<125$ & $<125$ & $<125$ & $<125$ & $<125$ & $<125$ & $<125$ \\
\hline Formate & $\mu \mathrm{g} / \mathrm{L}$ & U.S. & $2,500,000$ & SWEG & $<125$ & $<125$ & $<125$ & $<125$ & $<125$ & $<125$ & $<125$ & $<125$ \\
\hline Glycolate & $\mu \mathrm{g} / \mathrm{L}$ & U.S. & & & $<125$ & $<125$ & $<125$ & $<125$ & $<125$ & $<125$ & $<125$ & $<125$ \\
\hline Glyoxylate & $\mu \mathrm{g} / \mathrm{L}$ & U.S. & & & $<125$ & $<125$ & $<125$ & $<125$ & $<125$ & $<125$ & $<125$ & $<125$ \\
\hline Lactate & $\mu \mathrm{g} / \mathrm{L}$ & U.S. & & & $<1000$ & $<1000$ & $<1000$ & $<1000$ & $<1000$ & $<1000$ & $<1000$ & $<1000$ \\
\hline Oxalate & $\mu \mathrm{g} / \mathrm{L}$ & U.S. & & & $<125$ & $<125$ & MI & $<125$ & $<125$ & $<125$ & $<125$ & $<125$ \\
\hline Propionate & $\mu \mathrm{g} / \mathrm{L}$ & U.S. & & & $<125$ & $<125$ & $<125$ & $<125$ & $<125$ & $<125$ & $<125$ & $<125$ \\
\hline \begin{tabular}{|l} 
Aldehydes \\
\end{tabular} & & & & & & & & & & & & \\
\hline Formaldehyde & $\mu \mathrm{g} / \mathrm{L}$ & U.S. & $12,000 / 1,000$ & SWEG/EPA HA & $<2$ & 20 & $<2$ & $<2$ & $<2$ & 3 & 4 & 4 \\
\hline & & & & & & & & & & & & \\
\hline Amines (CE) & & & & & & & & & & & & \\
\hline Ethylamine & $\mu \mathrm{g} / \mathrm{L}$ & U.S. & \begin{tabular}{|l|} 
Monoalkylamines 2000 \\
\end{tabular} & SWEG & $<125$ & $<125$ & $<125$ & $<125$ & $<125$ & $<125$ & $<125$ & $<125$ \\
\hline Methylamine & $\mu \mathrm{g} / \mathrm{L}$ & U.S. & \begin{tabular}{|l|} 
Monoalkylamines 2000 \\
\end{tabular} & SWEG & $<125$ & $<125$ & $<125$ & $<125$ & $<125$ & $<125$ & $<125$ & $<125$ \\
\hline n-Propylamine & $\mu \mathrm{g} / \mathrm{L}$ & U.S. & \begin{tabular}{|l|} 
Monoalkylamines 2000 \\
\end{tabular} & SWEG & $<125$ & $<125$ & $<125$ & $<125$ & $<125$ & $<125$ & $<125$ & $<125$ \\
\hline Trimethylamine & $\mu \mathrm{g} / \mathrm{L}$ & U.S. & \begin{tabular}{|l|} 
Trialkylamines 400 \\
\end{tabular} & SWEG & $<125$ & $<125$ & $<125$ & $<125$ & $<125$ & $<125$ & $<125$ & $<125$ \\
\hline Non-volatiles (LC/UV-VIS) & & & & & & & & & & & & \\
\hline Urea & $\mu \mathrm{g} / \mathrm{L}$ & U.S. & & & $<800$ & $<800$ & $<800$ & $<800$ & $<800$ & $<800$ & $<800$ & $<800$ \\
\hline Caprolactam & $\mu \mathrm{g} / \mathrm{L}$ & U.S. & 100,000 & SWEG & $<8$ & $<4$ & $<4$ & $<4$ & $<4$ & $<16$ & $<8$ & $<8$ \\
\hline & & & & & & & & & & & & \\
\hline Organic Carbon Recovery & percent & U.S. & & & 0.20 & 2.25 & 0.21 & 0.24 & 0.27 & 0.18 & 0.36 & 0.37 \\
\hline Unaccounted Organic Carbon & $\mathrm{mg} / \mathrm{L}$ & U.S. & & & 2.69 & 0.35 & 3.37 & 3.25 & 3.47 & 3.52 & 0.48 & 0.47 \\
\hline
\end{tabular}


Appendix 3. ISS WPA RIP and PWD Summary for Expeditions 21 through 25

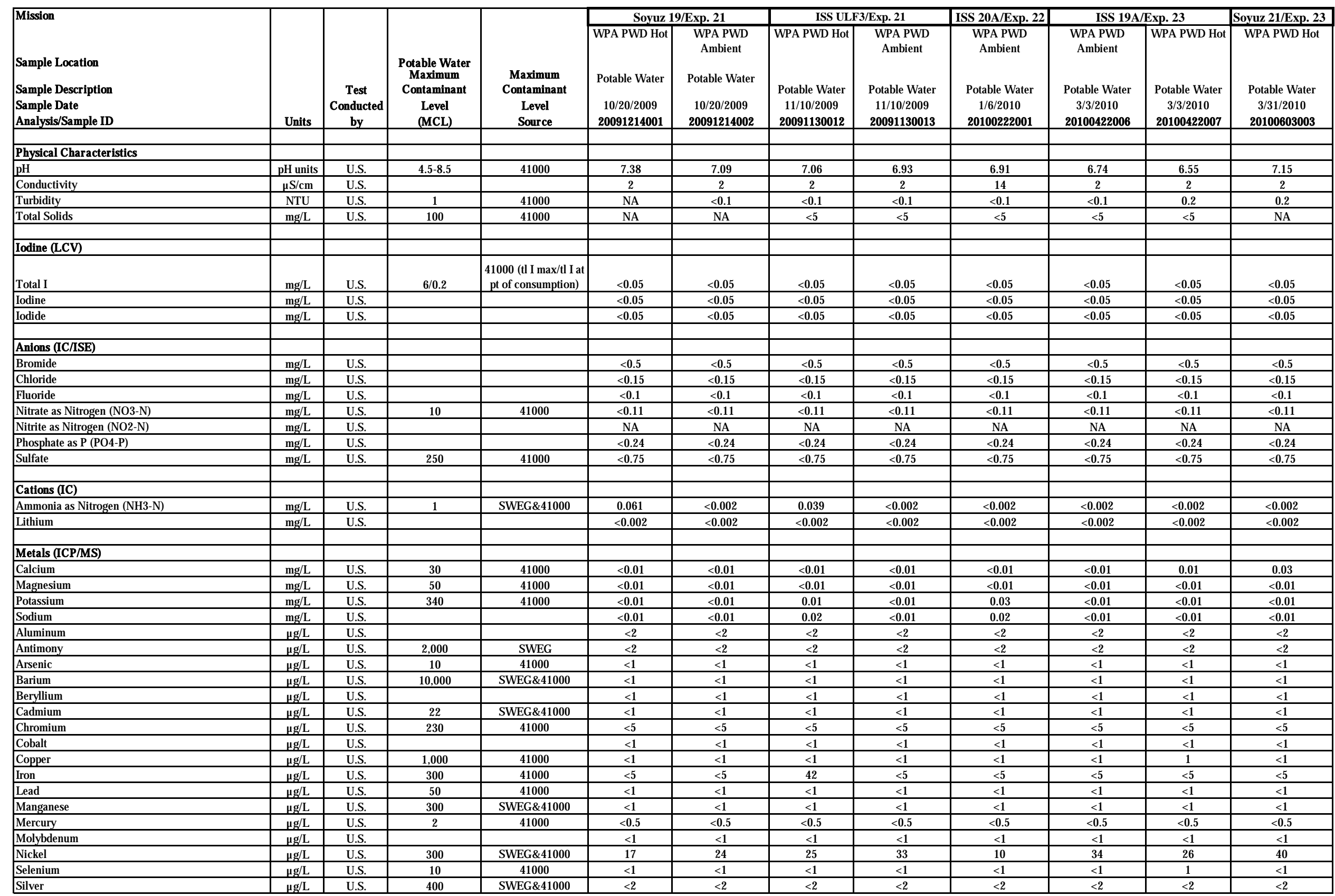


Appendix 3. ISS WPA RIP and PWD Summary for Expeditions 21 through 25

\begin{tabular}{|c|c|c|c|c|c|c|c|c|c|c|c|c|}
\hline \multirow[b]{2}{*}{\begin{tabular}{|l} 
Mission \\
Sample Location \\
Sample Description \\
Sample Date \\
Analysis/Sample ID
\end{tabular}} & \multirow[b]{2}{*}{ Units } & \multirow[b]{2}{*}{$\begin{array}{c}\text { Test } \\
\text { Conducted } \\
\text { by }\end{array}$} & \multirow[b]{2}{*}{$\begin{array}{c}\text { Potable W ater } \\
\text { Maximum } \\
\text { Contaminant } \\
\text { Level } \\
\text { (MCL) }\end{array}$} & \multirow[b]{2}{*}{$\begin{array}{c}\text { Maximum } \\
\text { Contaminant } \\
\text { Level } \\
\text { Source }\end{array}$} & \multicolumn{2}{|c|}{ Soyuz 19/Exp. 21} & \multicolumn{2}{|c|}{ ISS ULF3/Exp. 21} & \multirow[b]{2}{*}{\begin{tabular}{|c|} 
ISS 20A/Exp. 22 \\
W PA PWD \\
Ambient \\
\\
Potable W ater \\
$1 / 6 / 2010$ \\
20100222001 \\
\end{tabular}} & \multicolumn{2}{|c|}{ ISS 19A/Exp. 23} & \multirow[b]{2}{*}{\begin{tabular}{|c|} 
Soyuz 21/Exp. 23 \\
W PA PW D Hot \\
\\
Potable Water \\
$3 / 31 / 2010$ \\
20100603003 \\
\end{tabular}} \\
\hline & & & & & \begin{tabular}{|c|} 
W PA PWD Hot \\
\\
Potable W ater \\
10/20/2009 \\
20091214001 \\
\end{tabular} & $\begin{array}{c}\text { WPA PWD } \\
\text { Ambient } \\
\text { Potable Water } \\
\text { 10/20/2009 } \\
20091214002\end{array}$ & \begin{tabular}{|c|} 
W PA PW D Hot \\
\\
Potable Water \\
$11 / 10 / 2009$ \\
20091130012 \\
\end{tabular} & $\begin{array}{l}\text { WPA PWD } \\
\text { Ambient } \\
\\
\text { Potable W ater } \\
11 / 10 / 2009 \\
20091130013\end{array}$ & & $\begin{array}{c}\text { WPA PWD } \\
\text { Ambient } \\
\\
\text { Potable Water } \\
3 / 3 / 2010 \\
20100422006\end{array}$ & \begin{tabular}{|c|} 
WPA PWD Hot \\
\\
Potable W ater \\
$3 / 3 / 2010$ \\
20100422007 \\
\end{tabular} & \\
\hline Zinc & $\mu \mathrm{g} / \mathrm{L}$ & U.S. & 2,000 & SWEG\&41000 & $<1$ & $<1$ & $<1$ & $<1$ & $<1$ & $<1$ & $<1$ & $<1$ \\
\hline \multicolumn{13}{|l|}{ Silicon (ICP/MS) } \\
\hline \begin{tabular}{|l} 
Silicon (ICP/MS) \\
\end{tabular} & & & & & NA & NA & NA & 43 & 22 & 136 & 185 & $\mathrm{NA}$ \\
\hline \multicolumn{13}{|l|}{ Total Organic Carbon (Sievers) } \\
\hline \begin{tabular}{|l} 
Total Inorganic Carbon \\
\end{tabular} & $\mathrm{mg} / \mathrm{L}$ & U.S. & & & 0.71 & 0.73 & 1.09 & 1.08 & 1.22 & 1.34 & 1.23 & 1.70 \\
\hline Total Organic Carbon & $\mathrm{mg} / \mathrm{L}$ & U.S. & 3 & 41000 & 0.30 & 0.22 & 0.22 & 0.14 & 0.16 & 0.17 & 0.16 & 0.26 \\
\hline \multicolumn{13}{|l|}{ Volatile Organics } \\
\hline \begin{tabular}{|l} 
Acetone \\
\end{tabular} & $\mu \mathrm{g} / \mathrm{L}$ & U.S. & 15,000 & SWEG & $<2$ & $<2$ & $<2$ & $<2$ & $<2$ & 39 & 11 & $<2$ \\
\hline \begin{tabular}{|l|} 
Acryloniltrile \\
\end{tabular} & $\mu \mathrm{g} / \mathrm{L}$ & U.S. & & & $<2$ & $<2$ & $<2$ & $<2$ & $<2$ & $<2$ & $<2$ & $<2$ \\
\hline Allyl chloride (3-Chloropropene) & $\mu \mathrm{g} / \mathrm{L}$ & U.S. & & & $<2$ & $<2$ & $<2$ & $<2$ & $<2$ & $<2$ & $<2$ & $<2$ \\
\hline Benzene & $\mu \mathrm{g} / \mathrm{L}$ & U.S. & $70 / 5$ & SWEG/EPA & $<0.4$ & $<0.4$ & $<0.4$ & $<0.4$ & $<0.4$ & $<0.4$ & $<0.4$ & $<0.4$ \\
\hline Bromobenzene & $\mu \mathrm{g} / \mathrm{L}$ & U.S. & & & $<0.4$ & $<0.4$ & $<0.4$ & $<0.4$ & $<0.4$ & $<0.4$ & $<0.4$ & $<0.4$ \\
\hline Bromochloromethane & $\mu \mathrm{g} / \mathrm{L}$ & U.S. & 90 & EPA HA & $<4$ & $<4$ & $<4$ & $<4$ & $<4$ & $<4$ & $<4$ & $<4$ \\
\hline \begin{tabular}{|l} 
Bromoform \\
\end{tabular} & $\mu \mathrm{g} / \mathrm{L}$ & U.S. & THM 80 & EPA & $<2$ & $<2$ & $<2$ & $<2$ & $<2$ & $<2$ & $<2$ & $<2$ \\
\hline Bromomethane & $\mu \mathrm{g} / \mathrm{L}$ & U.S. & 10 & EPA HA & $<2$ & $<2$ & $<2$ & $<2$ & $<2$ & $<2$ & $<2$ & $<2$ \\
\hline 2-Butanone (Methyl ethyl ketone) & $\mu \mathrm{g} / \mathrm{L}$ & U.S. & $54000 / 4000$ & SWEG/EPA & $<2$ & $<2$ & $<2$ & $<2$ & $<2$ & $<2$ & $<2$ & $<2$ \\
\hline n-Butylbenzene & $\mu \mathrm{g} / \mathrm{L}$ & U.S. & & & $<0.4$ & $<0.4$ & $<0.4$ & $<0.4$ & $<0.4$ & $<0.4$ & $<0.4$ & $<0.4$ \\
\hline sec-Butylbenzene & $\mu \mathrm{g} / \mathrm{L}$ & U.S. & & & $<0.4$ & $<0.4$ & $<0.4$ & $<0.4$ & $<0.4$ & $<0.4$ & $<0.4$ & $<0.4$ \\
\hline tert-Butylbenzene & $\mu \mathrm{g} / \mathrm{L}$ & U.S. & & & $<0.4$ & $<0.4$ & $<0.4$ & $<0.4$ & $<0.4$ & $<0.4$ & $<0.4$ & $<0.4$ \\
\hline Carbon disulfide & $\mu \mathrm{g} / \mathrm{L}$ & U.S. & & & $<2$ & $<2$ & $<2$ & $<2$ & $<2$ & $<2$ & $<2$ & $<2$ \\
\hline Carbon tetrachloride & $\mu \mathrm{g} / \mathrm{L}$ & U.S. & 5 & EPA & $<0.4$ & $<0.4$ & $<0.4$ & $<0.4$ & $<0.4$ & $<0.4$ & $<0.4$ & $<0.4$ \\
\hline \begin{tabular}{|l} 
Chloroacetonitrile \\
\end{tabular} & $\mu \mathrm{g} / \mathrm{L}$ & U.S. & & & $<10$ & $<10$ & $<10$ & $<10$ & $<10$ & $<10$ & $<10$ & $<10$ \\
\hline \begin{tabular}{|l|} 
Chlorobenzene \\
\end{tabular} & $\mu \mathrm{g} / \mathrm{L}$ & U.S. & 100 & EPA & $<0.4$ & $<0.4$ & $<0.4$ & $<0.4$ & $<0.4$ & $<0.4$ & $<0.4$ & $<0.4$ \\
\hline 1-Chlorobutane (Butyl chloride) & $\mu \mathrm{g} / \mathrm{L}$ & U.S. & & & $<0.4$ & $<0.4$ & $<0.4$ & $<0.4$ & $<0.4$ & $<0.4$ & $<0.4$ & $<0.4$ \\
\hline Chloroethane & $\mu \mathrm{g} / \mathrm{L}$ & U.S. & & & $<2$ & $<2$ & $<2$ & $<2$ & $<2$ & $<2$ & $<2$ & $<2$ \\
\hline \begin{tabular}{|l|} 
Chloroform \\
\end{tabular} & $\mu \mathrm{g} / \mathrm{L}$ & U.S. & $6,500 / \mathrm{THM} 80$ & SWEG/EPA & $<0.4$ & $<0.4$ & $<0.4$ & $<0.4$ & $<0.4$ & $<0.4$ & $<0.4$ & $<0.4$ \\
\hline Chloromethane & $\mu \mathrm{g} / \mathrm{L}$ & U.S. & 30 & EPA HA & $<2$ & $<2$ & $<2$ & $<2$ & $<2$ & $<2$ & $<2$ & $<2$ \\
\hline 2-Chlorotoluene & $\mu \mathrm{g} / \mathrm{L}$ & U.S. & 100 & EPA HA & $<0.4$ & $<0.4$ & $<0.4$ & $<0.4$ & $<0.4$ & $<0.4$ & $<0.4$ & $<0.4$ \\
\hline 4-Chlorotoluene & $\mu \mathrm{g} / \mathrm{L}$ & U.S. & 100 & EPA HA & $<0.4$ & $<0.4$ & $<0.4$ & $<0.4$ & $<0.4$ & $<0.4$ & $<0.4$ & $<0.4$ \\
\hline Dibromochloromethane & $\mu \mathrm{g} / \mathrm{L}$ & U.S. & THM 80 & EPA & $<0.4$ & $<0.4$ & $<0.4$ & $<0.4$ & $<0.4$ & $<0.4$ & $<0.4$ & $<0.4$ \\
\hline 1,2-Dibromo-3-chloropropane (DBCP) & $\mu \mathrm{g} / \mathrm{L}$ & U.S. & 0.2 & EPA & $<2$ & $<2$ & $<2$ & $<2$ & $<2$ & $<2$ & $<2$ & $<2$ \\
\hline 1,2-Dibromoethane (EDB) & $\mu \mathrm{g} / \mathrm{L}$ & U.S. & 0.05 & EPA & $<0.4$ & $<0.4$ & $<0.4$ & $<0.4$ & $<0.4$ & $<0.4$ & $<0.4$ & $<0.4$ \\
\hline \begin{tabular}{|l} 
Dibromomethane \\
\end{tabular} & $\mu \mathrm{g} / \mathrm{L}$ & U.S. & & & $<0.4$ & $<0.4$ & $<0.4$ & $<0.4$ & $<0.4$ & $<0.4$ & $<0.4$ & $<0.4$ \\
\hline 1,2-Dichlorobenzene & $\mu \mathrm{g} / \mathrm{L}$ & U.S. & 600 & EPA & $<0.4$ & $<0.4$ & $<0.4$ & $<0.4$ & $<0.4$ & $<0.4$ & $<0.4$ & $<0.4$ \\
\hline 1,3-Dichlorobenzene & $\mu \mathrm{g} / \mathrm{L}$ & U.S. & 600 & EPA HA & $<0.4$ & $<0.4$ & $<0.4$ & $<0.4$ & $<0.4$ & $<0.4$ & $<0.4$ & $<0.4$ \\
\hline 1,1-Dichloroethane & $\mu \mathrm{g} / \mathrm{L}$ & U.S. & & & $<0.4$ & $<0.4$ & $<0.4$ & $<0.4$ & $<0.4$ & $<0.4$ & $<0.4$ & $<0.4$ \\
\hline 1,2-Dichloroethane & $\mu \mathrm{g} / \mathrm{L}$ & U.S. & 5 & EPA & $<0.4$ & $<0.4$ & $<0.4$ & $<0.4$ & $<0.4$ & $<0.4$ & $<0.4$ & $<0.4$ \\
\hline 1,1-Dichloroethene & $\mu \mathrm{g} / \mathrm{L}$ & U.S. & 7 & EPA & $<0.4$ & $<0.4$ & $<0.4$ & $<0.4$ & $<0.4$ & $<0.4$ & $<0.4$ & $<0.4$ \\
\hline cis1,2-Dichloroethene & $\mu \mathrm{g} / \mathrm{L}$ & U.S. & 70 & EPA & $<0.4$ & $<0.4$ & $<0.4$ & $<0.4$ & $<0.4$ & $<0.4$ & $<0.4$ & $<0.4$ \\
\hline \begin{tabular}{|l|} 
trans-1,2-Dichloroethene \\
\end{tabular} & $\mu \mathrm{g} / \mathrm{L}$ & U.S. & 100 & EPA & $<0.4$ & $<0.4$ & $<0.4$ & $<0.4$ & $<0.4$ & $<0.4$ & $<0.4$ & $<0.4$ \\
\hline 1,2-Dichloropropane & $\mu \mathrm{g} / \mathrm{L}$ & U.S. & 5 & EPA & $<0.4$ & $<0.4$ & $<0.4$ & $<0.4$ & $<0.4$ & $<0.4$ & $<0.4$ & $<0.4$ \\
\hline
\end{tabular}

NA=Not analyzed;

SWEG - 1000 days (11-2008) 


\begin{tabular}{|c|c|c|c|c|c|c|c|c|c|c|c|c|}
\hline \multirow{2}{*}{ Mission } & \multirow[b]{5}{*}{ Units } & \multirow{5}{*}{$\begin{array}{c}\text { Test } \\
\text { Conducted } \\
\text { by }\end{array}$} & \multirow{5}{*}{$\begin{array}{c}\text { Potable Water } \\
\text { Maximum } \\
\text { Contaminant } \\
\text { Level } \\
\text { (MCL) }\end{array}$} & \multirow{5}{*}{$\begin{array}{c}\text { Maximum } \\
\text { Contaminant } \\
\text { Level } \\
\text { Source }\end{array}$} & \multicolumn{2}{|c|}{ Soyuz 19/Exp. 21} & \multicolumn{2}{|c|}{ ISS ULF3/Exp. 21} & \multirow{5}{*}{\begin{tabular}{|c|} 
ISS 20A/Exp. 22 \\
W PA PWD \\
Ambient \\
\\
Potable Water \\
$1 / 6 / 2010$ \\
20100222001 \\
\end{tabular}} & \multicolumn{2}{|c|}{ ISS 19A/Exp. 23} & \multirow{5}{*}{\begin{tabular}{|c|} 
Soyuz 21/Exp. 23 \\
W PA PW D Hot \\
\\
\\
Potable Water \\
$3 / 31 / 2010$ \\
20100603003 \\
\end{tabular}} \\
\hline & & & & & \begin{tabular}{|l|} 
W PA PWD Hot \\
\end{tabular} & $\begin{array}{c}\text { WPA PWD } \\
\text { Ambient }\end{array}$ & WPA PWD Hot & $\begin{array}{c}\text { WPA PWD } \\
\text { Ambient }\end{array}$ & & $\begin{array}{c}\text { WPA PWD } \\
\text { Ambient }\end{array}$ & \begin{tabular}{|l|} 
WPA PWD Hot \\
\end{tabular} & \\
\hline Sample Location & & & & & Potable W ater & Potable Water & (2. & & & & & \\
\hline $\begin{array}{l}\text { Sample Description } \\
\text { Sample Date }\end{array}$ & & & & & $10 / 20 / 2009$ & 10/20/2009 & \begin{tabular}{|c|} 
Potable W ater \\
$11 / 10 / 2009$
\end{tabular} & $\begin{array}{c}\text { Potable Water } \\
\text { 11/10/2009 }\end{array}$ & & $\begin{array}{l}\text { Potable W ater } \\
\text { 3/3/2010 }\end{array}$ & \begin{tabular}{|c|} 
Potable $W$ ater \\
$3 / 3 / 2010$
\end{tabular} & \\
\hline Analysis/Sample ID & & & & & 20091214001 & 20091214002 & 20091130012 & & & 20100422006 & 20100422007 & \\
\hline 1,3-Dichloropropane & $\mu \mathrm{g} / \mathrm{L}$ & U.S. & & & $<0.4$ & $<0.4$ & $<0.4$ & $<0.4$ & $<0.4$ & $<0.4$ & $<0.4$ & $<0.4$ \\
\hline 2,2-Dichloropropane & $\mu \mathrm{g} / \mathrm{L}$ & U.S. & & & $<0.4$ & $<0.4$ & $<0.4$ & $<0.4$ & $<0.4$ & $<0.4$ & $<0.4$ & $<0.4$ \\
\hline 1,1-Dichloropropanone & $\mu \mathrm{g} / \mathrm{L}$ & U.S. & & & $<2$ & $<2$ & $<2$ & $<2$ & $<2$ & $<2$ & $<2$ & $<2$ \\
\hline 1,1-Dichloropropene & $\mu \mathrm{g} / \mathrm{L}$ & U.S. & & & $<0.4$ & $<0.4$ & $<0.4$ & $<0.4$ & $<0.4$ & $<0.4$ & $<0.4$ & $<0.4$ \\
\hline trans-1,3-Dichloropropene & $\mu \mathrm{g} / \mathrm{L}$ & U.S. & & & $<2$ & $<2$ & $<2$ & $<2$ & $<2$ & $<2$ & $<2$ & $<2$ \\
\hline \begin{tabular}{|l|} 
Diethyl ether \\
\end{tabular} & $\mu \mathrm{g} / \mathrm{L}$ & U.S. & & & $<2$ & $<2$ & $<2$ & $<2$ & $<2$ & $<2$ & $<2$ & $<2$ \\
\hline Ethylbenzene & $\mu \mathrm{g} / \mathrm{L}$ & U.S. & 700 & EPA & $<0.4$ & $<0.4$ & $<0.4$ & $<0.4$ & $<0.4$ & $<0.4$ & $<0.4$ & $<0.4$ \\
\hline \begin{tabular}{|l|} 
Ethyl methacrylate \\
\end{tabular} & $\mu \mathrm{g} / \mathrm{L}$ & U.S. & & & $<2$ & $<2$ & $<2$ & $<2$ & $<2$ & $<2$ & $<2$ & $<2$ \\
\hline Hexachlorobutadiene & $\mu \mathrm{g} / \mathrm{L}$ & U.S. & 1 & EPA HA & $<2$ & $<2$ & $<2$ & $<2$ & $<2$ & $<2$ & $<2$ & $<2$ \\
\hline \begin{tabular}{|l|} 
Hexachloroethane \\
\end{tabular} & $\mu \mathrm{g} / \mathrm{L}$ & U.S. & 1 & EPA HA & $<2$ & $<2$ & $<2$ & $<2$ & $<2$ & $<2$ & $<2$ & $<2$ \\
\hline 2-Hexanone & $\mu \mathrm{g} / \mathrm{L}$ & U.S. & & & $<2$ & $<2$ & $<2$ & $<2$ & $<2$ & $<2$ & $<2$ & $<2$ \\
\hline Iodomethane & $\mu \mathrm{g} / \mathrm{L}$ & U.S. & & & $<2$ & $<2$ & $<2$ & $<2$ & $<2$ & $<2$ & $<2$ & $<2$ \\
\hline Isopropylbenzene (Cumene) & $\mu \mathrm{g} / \mathrm{L}$ & U.S. & 4,000 & EPA DWEL & $<0.4$ & $<0.4$ & $<0.4$ & $<0.4$ & $<0.4$ & $<0.4$ & $<0.4$ & $<0.4$ \\
\hline 4-Isopropyltoluene (Cymene) & $\mu \mathrm{g} / \mathrm{L}$ & U.S. & & & $<0.4$ & $<0.4$ & $<0.4$ & $<0.4$ & $<0.4$ & $<0.4$ & $<0.4$ & $<0.4$ \\
\hline Methacrylonitrile & $\mu \mathrm{g} / \mathrm{L}$ & U.S. & & & $<2$ & $<2$ & $<2$ & $<2$ & $<2$ & $<2$ & $<2$ & $<2$ \\
\hline Methyl-t-butylether (MTBE) & $\mu \mathrm{g} / \mathrm{L}$ & U.S. & & & $<2$ & $<2$ & $<2$ & $<2$ & $<2$ & $<2$ & $<2$ & $<2$ \\
\hline Methylene chloride (Dichloromethane) & $\mu \mathrm{g} / \mathrm{L}$ & U.S. & $15,000 / 5$ & SWEG/EPA & $<0.4$ & $<0.4$ & $<0.4$ & $<0.4$ & $<0.4$ & $<0.4$ & $<0.4$ & $<0.4$ \\
\hline \begin{tabular}{|l|} 
Methyl methacrylate \\
\end{tabular} & $\mu \mathrm{g} / \mathrm{L}$ & U.S. & & & $<2$ & $<2$ & $<2$ & $<2$ & $<2$ & $<2$ & $<2$ & $<2$ \\
\hline 4-Methyl-2-pentanone & $\mu \mathrm{g} / \mathrm{L}$ & U.S. & & & $<0.4$ & $<0.4$ & $<0.4$ & $<0.4$ & $<0.4$ & $<0.4$ & $<0.4$ & $<0.4$ \\
\hline Naphthalene & $\mu \mathrm{g} / \mathrm{L}$ & U.S. & 100 & EPA HA & $<0.4$ & $<0.4$ & $<0.4$ & $<0.4$ & $<0.4$ & $<0.4$ & $<0.4$ & $<0.4$ \\
\hline Nitrobenzene & $\mu \mathrm{g} / \mathrm{L}$ & U.S. & & & $<2$ & $<2$ & $<2$ & $<2$ & $<2$ & $<2$ & $<2$ & $<2$ \\
\hline 2-Nitropropane & $\mu \mathrm{g} / \mathrm{L}$ & U.S. & & & $<2$ & $<2$ & $<2$ & $<2$ & $<2$ & $<2$ & $<2$ & $<2$ \\
\hline Pentachloroethane & $\mu \mathrm{g} / \mathrm{L}$ & U.S. & & & $<2$ & $<2$ & $<2$ & $<2$ & $<2$ & $<2$ & $<2$ & $<2$ \\
\hline Propionitrile (Ethyl cyanide) & $\mu \mathrm{g} / \mathrm{L}$ & U.S. & & & $<10$ & $<10$ & $<10$ & $<10$ & $<10$ & $<10$ & $<10$ & $<10$ \\
\hline n-Propylbenzene & $\mu \mathrm{g} / \mathrm{L}$ & U.S. & & & $<0.4$ & $<0.4$ & $<0.4$ & $<0.4$ & $<0.4$ & $<0.4$ & $<0.4$ & $<0.4$ \\
\hline Styrene & $\mu \mathrm{g} / \mathrm{L}$ & U.S. & 100 & EPA & $<0.4$ & $<0.4$ & $<0.4$ & $<0.4$ & $<0.4$ & $<0.4$ & $<0.4$ & $<0.4$ \\
\hline 1,1,1,2-Tetrachloroethane & $\mu \mathrm{g} / \mathrm{L}$ & U.S. & 70 & EPA HA & $<0.4$ & $<0.4$ & $<0.4$ & $<0.4$ & $<0.4$ & $<0.4$ & $<0.4$ & $<0.4$ \\
\hline 1,1,2,2-Tetrachloroethane & $\mu \mathrm{g} / \mathrm{L}$ & U.S. & 0.3 & EPA HA & $<0.4$ & $<0.4$ & $<0.4$ & $<0.4$ & $<0.4$ & $<0.4$ & $<0.4$ & $<0.4$ \\
\hline \begin{tabular}{|l|} 
Tetrachloroethene \\
\end{tabular} & $\mu \mathrm{g} / \mathrm{L}$ & U.S. & 5 & EPA & $<0.4$ & $<0.4$ & $<0.4$ & $<0.4$ & $<0.4$ & $<0.4$ & $<0.4$ & $<0.4$ \\
\hline Tetrahydrofuran & $\mu \mathrm{g} / \mathrm{L}$ & U.S. & & & $<2$ & $<2$ & $<2$ & $<2$ & $<2$ & $<2$ & $<2$ & $<2$ \\
\hline Toluene & $\mu \mathrm{g} / \mathrm{L}$ & U.S. & 1,000 & EPA & $<0.4$ & $<0.4$ & $<0.4$ & $<0.4$ & $<0.4$ & $<0.4$ & $<0.4$ & $<0.4$ \\
\hline 1,2,3-Trichlorobenzene & $\mu \mathrm{g} / \mathrm{L}$ & U.S. & & & $<0.4$ & $<0.4$ & $<0.4$ & $<0.4$ & $<0.4$ & $<0.4$ & $<0.4$ & $<0.4$ \\
\hline 1,2,4-Trichlorobenzene & $\mu \mathrm{g} / \mathrm{L}$ & U.S. & 70 & EPA & $<0.4$ & $<0.4$ & $<0.4$ & $<0.4$ & $<0.4$ & $<0.4$ & $<0.4$ & $<0.4$ \\
\hline 1,1,1-Trichloroethane & $\mu \mathrm{g} / \mathrm{L}$ & U.S. & 200 & EPA & $<0.4$ & $<0.4$ & $<0.4$ & $<0.4$ & $<0.4$ & $<0.4$ & $<0.4$ & $<0.4$ \\
\hline 1,1,2-Trichloroethane & $\mu \mathrm{g} / \mathrm{L}$ & U.S. & 5 & EPA & $<0.4$ & $<0.4$ & $<0.4$ & $<0.4$ & $<0.4$ & $<0.4$ & $<0.4$ & $<0.4$ \\
\hline Trichloroethene & $\mu \mathrm{g} / \mathrm{L}$ & U.S. & 5 & EPA & $<0.4$ & $<0.4$ & $<0.4$ & $<0.4$ & $<0.4$ & $<0.4$ & $<0.4$ & $<0.4$ \\
\hline Trichlorofluoromethane & $\mu \mathrm{g} / \mathrm{L}$ & U.S. & 2,000 & EPA HA & $<0.4$ & $<0.4$ & $<0.4$ & $<0.4$ & $<0.4$ & $<0.4$ & $<0.4$ & $<0.4$ \\
\hline Vinyl Acetate & $\mu \mathrm{g} / \mathrm{L}$ & U.S. & & & $<2$ & $<2$ & $<2$ & $<2$ & $<2$ & $<2$ & $<2$ & $<2$ \\
\hline Vinyl Chloride & $\mu \mathrm{g} / \mathrm{L}$ & U.S. & 2 & EPA & $<2$ & $<2$ & $<2$ & $<2$ & $<2$ & $<2$ & $<2$ & $<2$ \\
\hline m\&p-Xylene & $\mu \mathrm{g} / \mathrm{L}$ & U.S. & \begin{tabular}{|l|l} 
Total X ylenes 10,000 \\
\end{tabular} & EPA & $<0.4$ & $<0.4$ & $<0.4$ & $<0.4$ & $<0.4$ & $<0.4$ & $<0.4$ & $<0.4$ \\
\hline 0 -Xylene & $\mu \mathrm{g} / \mathrm{L}$ & U.S. & \begin{tabular}{|l|} 
Total $\mathrm{X}$ ylenes 10,000 \\
\end{tabular} & EPA & $<0.4$ & $<0.4$ & $<0.4$ & $<0.4$ & $<0.4$ & $<0.4$ & $<0.4$ & $<0.4$ \\
\hline & & & & & & & & & & & & \\
\hline
\end{tabular}




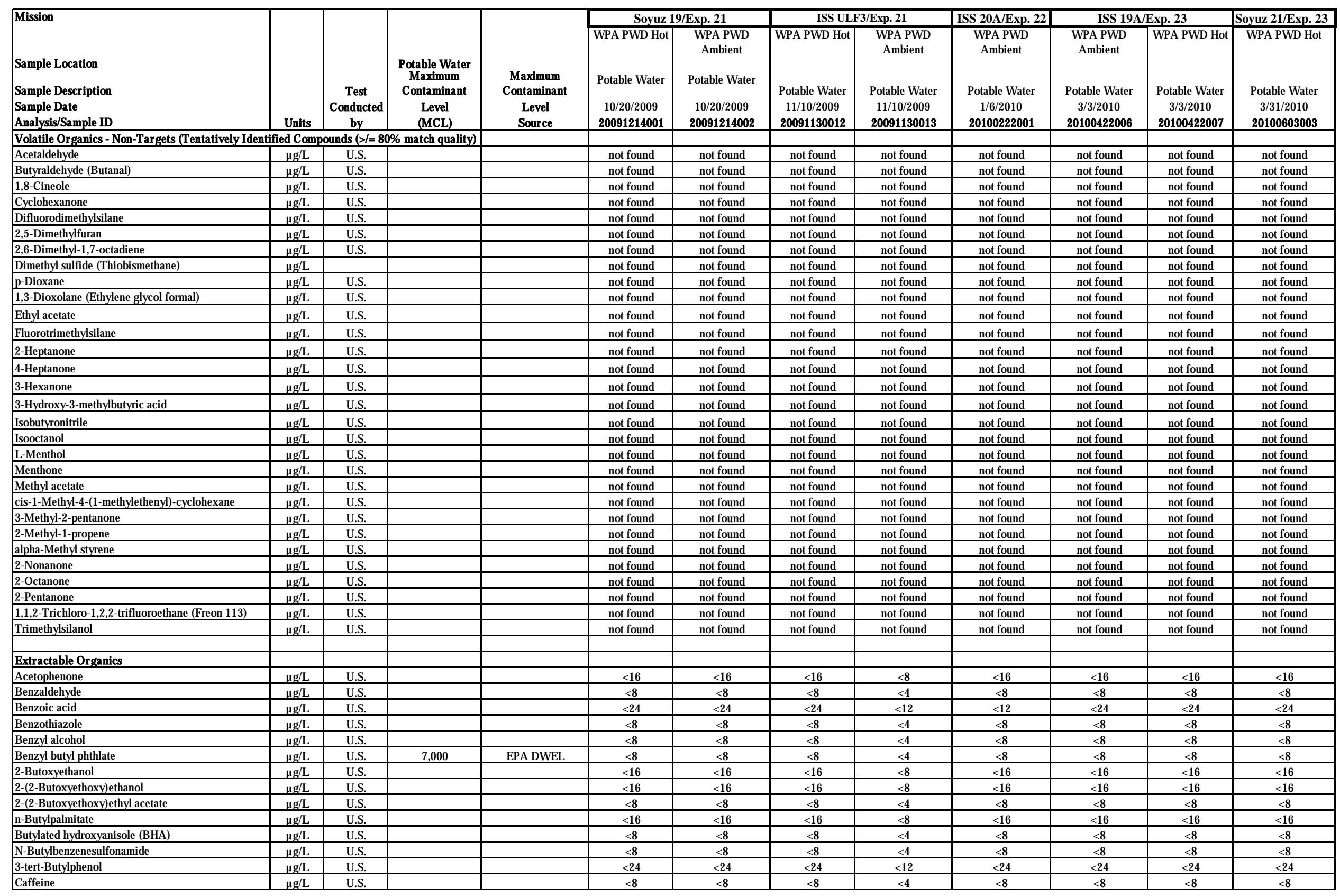




\begin{tabular}{|c|c|c|c|c|c|c|c|c|c|c|c|c|}
\hline \multirow[t]{2}{*}{ Mission } & \multirow[b]{5}{*}{ Units } & \multirow{5}{*}{$\begin{array}{c}\text { Test } \\
\text { Conducted } \\
\text { by }\end{array}$} & \multirow{5}{*}{$\begin{array}{c}\text { Potable Water } \\
\text { Maximum } \\
\text { Contaminant } \\
\text { Level } \\
\text { (MCL) }\end{array}$} & \multirow{5}{*}{$\begin{array}{c}\text { Maximum } \\
\text { Contaminant } \\
\text { Level } \\
\text { Source }\end{array}$} & \multicolumn{2}{|c|}{ Soyuz 19/Exp. 21} & \multicolumn{2}{|c|}{ ISS ULF3/Exp. 21} & \multirow{5}{*}{\begin{tabular}{|c|} 
ISS 20A/Exp. 22 \\
W PA PWD \\
Ambient \\
\\
Potable Water \\
$1 / 6 / 2010$ \\
20100222001 \\
\end{tabular}} & \multicolumn{2}{|c|}{ ISS 19A/Exp. 23} & \multirow{5}{*}{\begin{tabular}{|c} 
Soyuz 21/Exp. 23 \\
W PA PW D Hot \\
\\
Potable Water \\
$3 / 31 / 2010$ \\
20100603003 \\
\end{tabular}} \\
\hline & & & & & \begin{tabular}{|l|} 
W PA PWD Hot \\
\end{tabular} & $\begin{array}{c}\text { WPA PWD } \\
\text { Ambient }\end{array}$ & WPA PWD Hot & $\begin{array}{c}\text { WPA PWD } \\
\text { Ambient }\end{array}$ & & $\begin{array}{l}\text { WPA PWD } \\
\text { Ambient }\end{array}$ & \begin{tabular}{|l|} 
WPA PWD Hot \\
\end{tabular} & \\
\hline Sample Location & & & & & Potable W ater & Potable Water & (2. & & & & 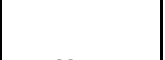 & \\
\hline $\begin{array}{l}\text { Sample Description } \\
\text { Sample Date }\end{array}$ & & & & & 10/20/2009 & 10/20/2009 & $\begin{array}{c}\text { Potable Water } \\
11 / 10 / 2009\end{array}$ & $\begin{array}{c}\text { Potable Water } \\
11 / 10 / 2009\end{array}$ & & $\begin{array}{c}\text { Potable W ater } \\
3 / 3 / 2010\end{array}$ & $\begin{array}{c}\text { Potable Water } \\
3 / 3 / 2010\end{array}$ & \\
\hline Analysis/Sample ID & & & & & 20091214001 & 20091214002 & 20091130012 & & & 20100422006 & 20100422007 & \\
\hline tris-2-Chloroethyl phosphate & $\mu \mathrm{g} / \mathrm{L}$ & U.S. & & & $<8$ & $<8$ & $<8$ & $<4$ & $<8$ & $<8$ & $<8$ & $<8$ \\
\hline \begin{tabular}{|l|} 
Cholesterol \\
\end{tabular} & $\mu \mathrm{g} / \mathrm{L}$ & U.S. & & & $<64$ & $<64$ & $<64$ & $<32$ & $<64$ & $<64$ & $<64$ & $<64$ \\
\hline 0-Cresol (2-Methylphenol) & $\mu \mathrm{g} / \mathrm{L}$ & U.S. & & & $<8$ & $<8$ & $<8$ & $<4$ & $<8$ & $<8$ & $<8$ & $<8$ \\
\hline Cyclododecane & $\mu \mathrm{g} / \mathrm{L}$ & U.S. & & & $<8$ & $<8$ & $<8$ & $<4$ & $<8$ & $<8$ & $<8$ & $<8$ \\
\hline Decanoic acid & $\mu \mathrm{g} / \mathrm{L}$ & U.S. & & & $<16$ & $<16$ & $<16$ & $<8$ & $<16$ & $<16$ & $<16$ & $<16$ \\
\hline 2,6-Di-t-butyl-1,4-benzoquinone & $\mu \mathrm{g} / \mathrm{L}$ & U.S. & & & $<8$ & $<8$ & $<8$ & $<4$ & $<8$ & $<8$ & $<8$ & $<8$ \\
\hline 2,4-Di-t-butylphenol & $\mu \mathrm{g} / \mathrm{L}$ & U.S. & & & $<8$ & $<8$ & $<8$ & $<4$ & $<8$ & $<8$ & $<8$ & $<8$ \\
\hline 1,4 Diacetylbenzene & $\mu \mathrm{g} / \mathrm{L}$ & U.S. & & & $<8$ & $<8$ & $<8$ & $<4$ & $<8$ & $<8$ & $<8$ & $<8$ \\
\hline N,N-Dibutylformamide & $\mu \mathrm{g} / \mathrm{L}$ & U.S. & & & $<8$ & $<8$ & $<8$ & $<4$ & $<8$ & $<8$ & $<8$ & $<8$ \\
\hline \begin{tabular}{|l|} 
Dibutyl phthalate \\
\end{tabular} & $\mu \mathrm{g} / \mathrm{L}$ & U.S. & $40,000 / 4,000$ & SWEG/EPA DWEL & $<8$ & $<8$ & $<8$ & $<4$ & $<8$ & $<8$ & $<8$ & $<8$ \\
\hline Dibutylamine & $\mu \mathrm{g} / \mathrm{L}$ & U.S. & Dialkylamines 300 & SWEG & $<8$ & $<8$ & $<8$ & $<4$ & $<8$ & $<8$ & $<8$ & $<8$ \\
\hline N,N-Diethyl-m-toluamide & $\mu \mathrm{g} / \mathrm{L}$ & U.S. & & & $<8$ & $<8$ & $<8$ & $<4$ & $<8$ & $<8$ & $<8$ & $<8$ \\
\hline Diethylphthalate & $\mu \mathrm{g} / \mathrm{L}$ & U.S. & 30,000 & EPA DWEL & $<8$ & $<8$ & $<8$ & $<4$ & $<8$ & $<8$ & $<8$ & $<8$ \\
\hline Diethylene glycol monoethyl ether & $\mu \mathrm{g} / \mathrm{L}$ & U.S. & & & $<8$ & $<8$ & $<8$ & $<4$ & $<8$ & $<8$ & $<8$ & $<8$ \\
\hline \begin{tabular}{|l|}
$\mathrm{N}, \mathrm{N}$-Diethylformamide \\
\end{tabular} & $\mu \mathrm{g} / \mathrm{L}$ & U.S. & & & $<24$ & $<24$ & $<24$ & $<12$ & $<24$ & $<24$ & $<24$ & $<24$ \\
\hline \begin{tabular}{|l} 
Diisopropyl adipate \\
\end{tabular} & $\mu \mathrm{g} / \mathrm{L}$ & U.S. & & & $<8$ & $<8$ & $<8$ & $<4$ & $<8$ & $<8$ & $<8$ & $<8$ \\
\hline Dimethyl phthalate & $\mu \mathrm{g} / \mathrm{L}$ & U.S. & & & $<8$ & $<8$ & $<8$ & $<4$ & $<8$ & $<8$ & $<8$ & $<8$ \\
\hline N,N-Dimethyl acetamide & $\mu \mathrm{g} / \mathrm{L}$ & U.S. & & & $<8$ & $<8$ & $<8$ & $<4$ & $<8$ & $<8$ & $<8$ & $<8$ \\
\hline N,N-Dimethylbenzylamine & $\mu \mathrm{g} / \mathrm{L}$ & U.S. & Dialkylamines 300 & SWEG & $<8$ & $<8$ & $<8$ & $<4$ & $<8$ & $<8$ & $<8$ & $<8$ \\
\hline \begin{tabular}{|l|}
$\mathrm{N}, \mathrm{N}$-Dimethylformamide \\
\end{tabular} & $\mu \mathrm{g} / \mathrm{L}$ & U.S. & & & $<16$ & $<16$ & $<16$ & $<8$ & $<16$ & $<16$ & $<16$ & $<16$ \\
\hline \begin{tabular}{|l|} 
Dipropylene glycol methyl ether \\
\end{tabular} & $\mu \mathrm{g} / \mathrm{L}$ & U.S. & & & $<8$ & $<8$ & $<8$ & $<4$ & $<8$ & $<8$ & $<8$ & $<8$ \\
\hline Dodecamethylcyclohexasiloxane & $\mu \mathrm{g} / \mathrm{L}$ & U.S. & & & $<8$ & $<8$ & $<8$ & $<4$ & $<8$ & $<8$ & $<8$ & $<8$ \\
\hline 2-Ethoxyethanol & $\mu \mathrm{g} / \mathrm{L}$ & U.S. & & & $<8$ & $<8$ & $<8$ & $<4$ & $<8$ & $<8$ & $<8$ & $<8$ \\
\hline 2-Ethyl-1-hexanol & $\mu \mathrm{g} / \mathrm{L}$ & U.S. & & & $<8$ & $<8$ & $<8$ & $<4$ & $<8$ & $<8$ & $<8$ & $<8$ \\
\hline 2-Ethylhexanoic acid & $\mu \mathrm{g} / \mathrm{L}$ & U.S. & & & $<8$ & $<8$ & $<8$ & $<4$ & $<8$ & $<8$ & $<8$ & $<8$ \\
\hline bis-2-Ethylhexyl adipate & $\mu \mathrm{g} / \mathrm{L}$ & U.S. & 400 & EPA & $<8$ & $<8$ & $<8$ & $<4$ & $<8$ & $<8$ & $<8$ & $<8$ \\
\hline bis-2-Ethylhexyl phthalate (Dioctyl phthlate) & $\mu \mathrm{g} / \mathrm{L}$ & U.S. & $20,000 / 6$ & SWEG/EPA & $<8$ & $<8$ & $<8$ & $<4$ & $<8$ & $<8$ & $<8$ & $<8$ \\
\hline 4-Ethylmorpholine & $\mu \mathrm{g} / \mathrm{L}$ & U.S. & & & $<8$ & $<8$ & $<8$ & $<4$ & $<8$ & $<8$ & $<8$ & $<8$ \\
\hline 1-Formylpiperidine & $\mu \mathrm{g} / \mathrm{L}$ & U.S. & & & $<8$ & $<8$ & $<8$ & $<4$ & $<8$ & $<8$ & $<8$ & $<8$ \\
\hline Heptanoic acid & $\mu \mathrm{g} / \mathrm{L}$ & U.S. & & & $<8$ & $<8$ & $<8$ & $<4$ & $<8$ & $<8$ & $<8$ & $<8$ \\
\hline 2-Heptanone & $\mu \mathrm{g} / \mathrm{L}$ & U.S. & & & $<8$ & $<8$ & $<8$ & $<4$ & $<8$ & $<8$ & $<8$ & $<8$ \\
\hline gamma-Hexalactone & $\mu \mathrm{g} / \mathrm{L}$ & U.S. & & & $<8$ & $<8$ & $<8$ & $<4$ & $<8$ & $<8$ & $<8$ & $<8$ \\
\hline Hexanoic acid & $\mu \mathrm{g} / \mathrm{L}$ & U.S. & & & $<16$ & $<16$ & $<16$ & $<8$ & $<16$ & $<16$ & $<16$ & $<16$ \\
\hline 2-Hexanol & $\mu \mathrm{g} / \mathrm{L}$ & U.S. & & & $<8$ & $<8$ & $<8$ & $<4$ & $<8$ & $<8$ & $<8$ & $<8$ \\
\hline \begin{tabular}{|l|} 
2-Hydroxybenzothiazole \\
\end{tabular} & $\mu \mathrm{g} / \mathrm{L}$ & U.S. & & & $<8$ & $<8$ & $<8$ & $<4$ & $<8$ & $<8$ & $<8$ & $<8$ \\
\hline Ibuprofen & $\mu \mathrm{g} / \mathrm{L}$ & U.S. & & & $<8$ & $<8$ & $<8$ & $<4$ & $<8$ & $<8$ & $<8$ & $<8$ \\
\hline Iodoform & $\mu \mathrm{g} / \mathrm{L}$ & U.S. & & & $<8$ & $<8$ & $<8$ & $<4$ & $<8$ & $<8$ & $<8$ & $<8$ \\
\hline Lauric acid (Dodecanoic acid) & $\mu \mathrm{g} / \mathrm{L}$ & U.S. & & & $<240$ & $<240$ & $<240$ & $<120$ & $<240$ & $<240$ & $<240$ & $<240$ \\
\hline p-Menth-1-en-8-ol (alpha-Terpineol) & $\mu \mathrm{g} / \mathrm{L}$ & U.S. & & & $<8$ & $<8$ & $<8$ & $<4$ & $<8$ & $<8$ & $<8$ & $<8$ \\
\hline 2-Mercaptobenzothiazole & $\mu \mathrm{g} / \mathrm{L}$ & U.S. & 30,000 & SWEG & $<80$ & $<80$ & $<80$ & $<40$ & $<80$ & $<80$ & $<80$ & $<80$ \\
\hline 2-Methyl-2,4-pentanediol & $\mu \mathrm{g} / \mathrm{L}$ & U.S. & & & $<8$ & $<8$ & $<8$ & $<4$ & $<8$ & $<8$ & $<8$ & $<8$ \\
\hline 1-Methyl-2-pyrrolidinone & $\mu \mathrm{g} / \mathrm{L}$ & U.S. & & & $<8$ & $<8$ & $<8$ & $<4$ & $<8$ & $<8$ & $<8$ & $<8$ \\
\hline Methyl-4-hydroxybenzoate & $\mu \mathrm{g} / \mathrm{L}$ & U.S. & & & $<8$ & $<8$ & $<8$ & $<4$ & $<8$ & $<8$ & $<8$ & $<8$ \\
\hline
\end{tabular}


Appendix 3. ISS WPA RIP and PWD Summary for Expeditions 21 through 25

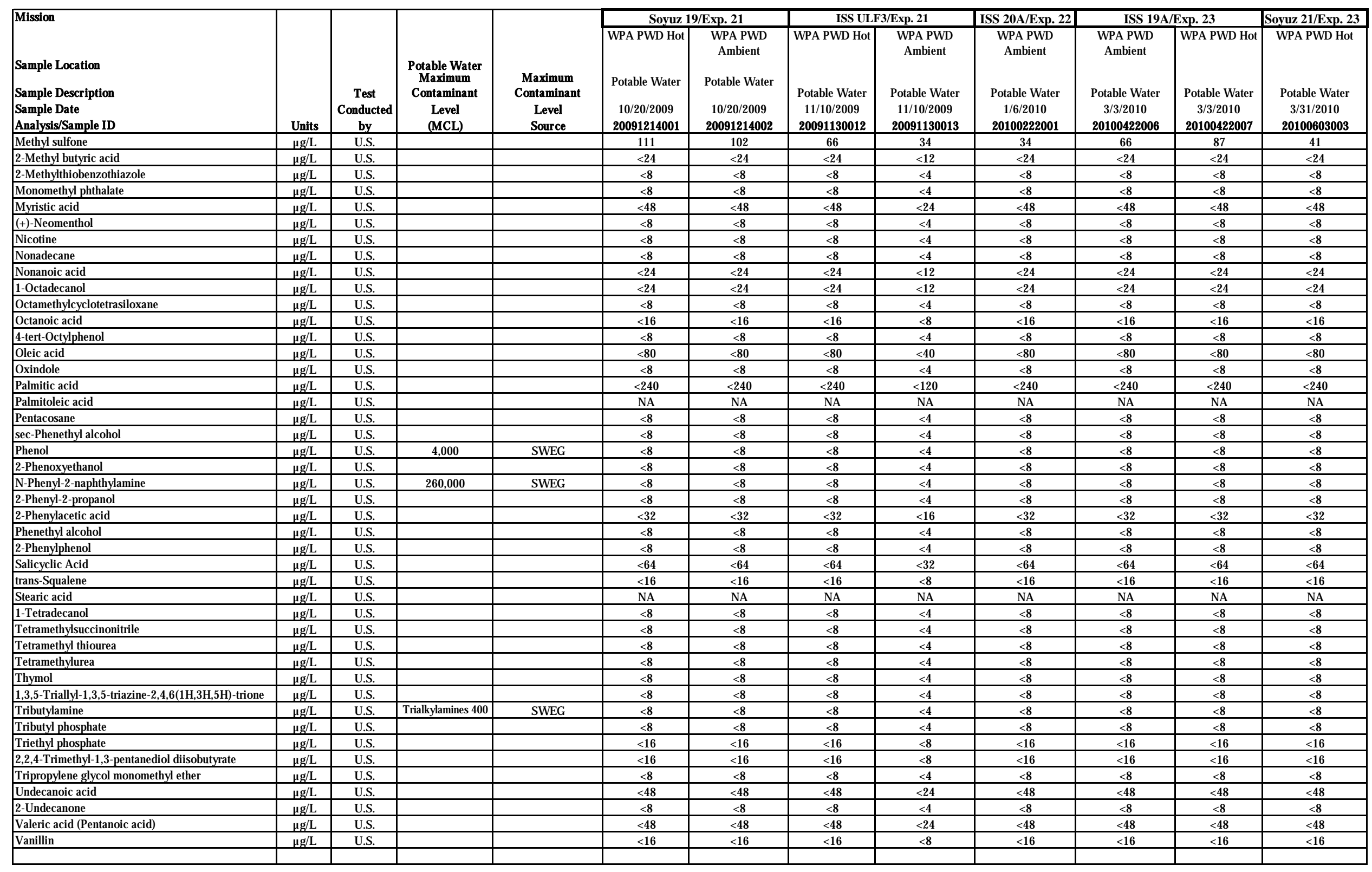


Appendix 3. ISS WPA RIP and PWD Summary for Expeditions 21 through 25

\begin{tabular}{|c|c|c|c|c|c|c|c|c|c|c|c|c|}
\hline Mission & \multirow[b]{2}{*}{ Units } & \multirow[b]{2}{*}{$\begin{array}{c}\text { Test } \\
\begin{array}{c}\text { Conducted } \\
\text { by }\end{array} \\
\end{array}$} & \multirow[b]{2}{*}{$\begin{array}{c}\text { Potable W ater } \\
\text { Maximum } \\
\text { Contaminant } \\
\text { Level } \\
\text { (MCL) } \\
\end{array}$} & \multirow[b]{2}{*}{$\begin{array}{c}\text { Maximum } \\
\text { Contaminant } \\
\text { Level } \\
\text { Source } \\
\end{array}$} & \multicolumn{2}{|c|}{ Soyuz 19/Exp. 21} & \multicolumn{2}{|c|}{ ISS ULF3/Exp. 21} & \multirow[b]{2}{*}{\begin{tabular}{|c|} 
ISS 20A/Exp. 22 \\
WPA PWD \\
Ambient \\
\\
Potable Water \\
$1 / 6 / 2010$ \\
20100222001 \\
\end{tabular}} & \multicolumn{2}{|c|}{ ISS 19A/Exp. 23} & \multirow[b]{2}{*}{\begin{tabular}{|c|} 
Soyuz 21/Exp. 23 \\
W PA PW D Hot \\
\\
Potable Water \\
$3 / 31 / 2010$ \\
20100603003 \\
\end{tabular}} \\
\hline $\begin{array}{l}\text { Sample Location } \\
\text { Sample Description } \\
\text { Sample Date } \\
\text { Analysis/Sample ID }\end{array}$ & & & & & \begin{tabular}{|c|} 
WPA PWD Hot \\
\\
Potable W ater \\
10/20/2009 \\
20091214001 \\
\end{tabular} & $\begin{array}{c}\text { WPA PWD } \\
\text { A mbient } \\
\text { Potable Water } \\
\text { 10/20/2009 } \\
20091214002\end{array}$ & \begin{tabular}{|c|} 
WPA PWD Hot \\
\\
Potable Water \\
$11 / 10 / 2009$ \\
20091130012 \\
\end{tabular} & $\begin{array}{c}\text { WPA PWD } \\
\text { Ambient } \\
\\
\text { Potable Water } \\
\text { 11/10/2009 } \\
20091130013\end{array}$ & & $\begin{array}{c}\text { WPA PWD } \\
\text { Ambient } \\
\\
\text { Potable Water } \\
3 / 3 / 2010 \\
20100422006\end{array}$ & \begin{tabular}{|c|} 
WPA PWD Hot \\
\\
Potable W ater \\
$3 / 3 / 2010$ \\
20100422007 \\
\end{tabular} & \\
\hline \multicolumn{13}{|c|}{\begin{tabular}{|l|} 
Acid Extractables-EPA 625 List \\
\end{tabular}} \\
\hline 4-Chloro-3-methylphenol & $\mu \mathrm{g} / \mathrm{L}$ & U.S. & & & $<16$ & $<16$ & $<16$ & $<8$ & $<16$ & $<16$ & $<16$ & $<16$ \\
\hline 2-Chlorophenol & $\mu \mathrm{g} / \mathrm{L}$ & U.S. & 40 & EPA HA & $<16$ & $<16$ & $<16$ & $<8$ & $<16$ & $<16$ & $<16$ & $<16$ \\
\hline 2,4-Dichlorophenol & $\mu \mathrm{g} / \mathrm{L}$ & U.S. & 20 & EPA HA & $<16$ & $<16$ & $<16$ & $<8$ & $<16$ & $<16$ & $<16$ & $<16$ \\
\hline 2,4-Dinitrophenol & $\mu \mathrm{g} / \mathrm{L}$ & U.S. & & & $<16$ & $<16$ & $<16$ & $<8$ & $<16$ & $<16$ & $<16$ & $<16$ \\
\hline 2-Methyl-4,6-dinitrophenol & $\mu \mathrm{g} / \mathrm{L}$ & U.S. & & & $<16$ & $<16$ & $<16$ & $<8$ & $<16$ & $<16$ & $<16$ & $<16$ \\
\hline 2-Nitrophenol & $\mu \mathrm{g} / \mathrm{L}$ & U.S. & & & $<16$ & $<16$ & $<16$ & $<8$ & $<16$ & $<16$ & $<16$ & $<16$ \\
\hline 4-Nitrophenol & $\mu \mathrm{g} / \mathrm{L}$ & U.S. & 60 & EPA HA & $<16$ & $<16$ & $<16$ & $<8$ & $<16$ & $<16$ & $<16$ & $<16$ \\
\hline \begin{tabular}{|l} 
Pentachlorophenol \\
\end{tabular} & $\mu \mathrm{g} / \mathrm{L}$ & U.S. & 1 & EPA & $<16$ & $<16$ & $<16$ & $<8$ & $<16$ & $<16$ & $<16$ & $<16$ \\
\hline Phenol & $\mu \mathrm{g} / \mathrm{L}$ & U.S. & $4,000 / 2,000$ & SWEG/EPA HA & $<8$ & $<8$ & $<8$ & $<4$ & $<8$ & $<8$ & $<8$ & $<8$ \\
\hline 2,4,5-Trichlorophenol & $\mu \mathrm{g} / \mathrm{L}$ & U.S. & & & $<16$ & $<16$ & $<16$ & $<8$ & $<16$ & $<16$ & $<16$ & $<16$ \\
\hline 2,4,6-Trichlorophenol & $\mu \mathrm{g} / \mathrm{L}$ & U.S. & 10 & EPA DWEL & $<16$ & $<16$ & $<16$ & $<8$ & $<16$ & $<16$ & $<16$ & $<16$ \\
\hline 4-Methylphenol & $\mu \mathrm{g} / \mathrm{L}$ & U.S. & & & $\mathrm{NA}$ & NA & $\mathrm{NA}$ & $\mathrm{NA}$ & $<4$ & $<8$ & $<8$ & $<8$ \\
\hline \multirow{2}{*}{\multicolumn{13}{|c|}{ Base/Neutral Extractables - EPA 625 List }} \\
\hline & & & & & & & & & & & & \\
\hline 3,3-Dichlorobenzidine & $\mu \mathrm{g} / \mathrm{L}$ & U.S. & & & $<16$ & $<16$ & $<16$ & $<8$ & $<16$ & $<16$ & $<16$ & $<16$ \\
\hline bis-(2-Ethylhexyl)phthalate & $\mu \mathrm{g} / \mathrm{L}$ & U.S. & $20,000 / 6$ & SWEG/EPA & $<8$ & $<8$ & $<8$ & $<4$ & $<8$ & $<8$ & $<8$ & $<8$ \\
\hline Benzyl butyl phthalate & $\mu \mathrm{g} / \mathrm{L}$ & U.S. & 7,000 & EPA DWEL & $<8$ & $<8$ & $<8$ & $<4$ & $<8$ & $<8$ & $<8$ & $<8$ \\
\hline Dibutylphthalate & $\mu \mathrm{g} / \mathrm{L}$ & U.S. & $40,000 / 4,000$ & SWEG/EPA DWEL & $<8$ & $<8$ & $<8$ & $<4$ & $<8$ & $<8$ & $<8$ & $<8$ \\
\hline Diethylphthalate & $\mu \mathrm{g} / \mathrm{L}$ & U.S. & 30,000 & EPA DWEL & $<8$ & $<8$ & $<8$ & $<4$ & $<8$ & $<8$ & $<8$ & $<8$ \\
\hline Dimethylphthalate & $\mu \mathrm{g} / \mathrm{L}$ & U.S. & & & $<8$ & $<8$ & $<8$ & $<4$ & $<8$ & $<8$ & $<8$ & $<8$ \\
\hline Di-n-octyl phthalate & $\mu \mathrm{g} / \mathrm{L}$ & U.S. & & & $<16$ & $<16$ & $<16$ & $<8$ & $<16$ & $<16$ & $<16$ & $<16$ \\
\hline N-Nitrosodimethylamine & $\mu \mathrm{g} / \mathrm{L}$ & U.S. & & & $<16$ & $<16$ & $<16$ & $<8$ & $<16$ & $<16$ & $<16$ & $<16$ \\
\hline N-Nitrosodiphenylamine & $\mu \mathrm{g} / \mathrm{L}$ & U.S. & & & $<16$ & $<16$ & $<16$ & $<8$ & $<16$ & $<16$ & $<16$ & $<16$ \\
\hline N-Nitrosodi-n-propylamine & $\mu \mathrm{g} / \mathrm{L}$ & U.S. & & & $<16$ & $<16$ & $<16$ & $<8$ & $<16$ & $<16$ & $<16$ & $<16$ \\
\hline 2,4-Dinitrotoluene & $\mu \mathrm{g} / \mathrm{L}$ & U.S. & 100 & EPA DWEL & $<16$ & $<16$ & $<16$ & $<8$ & $<16$ & $<16$ & $<16$ & $<16$ \\
\hline 2,6-Dinitrotoluene & $\mu \mathrm{g} / \mathrm{L}$ & U.S. & 40 & EPA DWEL & $<16$ & $<16$ & $<16$ & $<8$ & $<16$ & $<16$ & $<16$ & $<16$ \\
\hline Isophorone & $\mu \mathrm{g} / \mathrm{L}$ & U.S. & 100 & EPA HA & $<8$ & $<8$ & $<8$ & $<4$ & $<8$ & $<8$ & $<8$ & $<8$ \\
\hline Nitrobenzene & $\mu \mathrm{g} / \mathrm{L}$ & U.S. & & & $<16$ & $<16$ & $<16$ & $<8$ & $<16$ & $<16$ & $<16$ & $<16$ \\
\hline Acenaphthene & $\mu \mathrm{g} / \mathrm{L}$ & U.S. & 2000 & EPA DWEL & $<16$ & $<16$ & $<16$ & $<8$ & $<16$ & $<16$ & $<16$ & $<16$ \\
\hline Acenaphthylene & $\mu \mathrm{g} / \mathrm{L}$ & U.S. & & & $<16$ & $<16$ & $<16$ & $<8$ & $\mathrm{NA}$ & $<16$ & $<16$ & $<16$ \\
\hline \begin{tabular}{|l|} 
Anthracene \\
\end{tabular} & $\mu \mathrm{g} / \mathrm{L}$ & U.S. & 10,000 & EPA DWEL & $<16$ & $<16$ & $<16$ & $<8$ & $<16$ & $<16$ & $<16$ & $<16$ \\
\hline Benzo(a)anthracene & $\mu \mathrm{g} / \mathrm{L}$ & U.S. & & & $<16$ & $<16$ & $<16$ & $<8$ & $<16$ & $<16$ & $<16$ & $<16$ \\
\hline Benzo(a)pyrene & $\mu \mathrm{g} / \mathrm{L}$ & U.S. & 0.2 & EPA & $<10$ & $<10$ & $<10$ & $<5$ & $<10$ & $<10$ & $<10$ & $<10$ \\
\hline Benzo(b)fluoranthene & $\mu \mathrm{g} / \mathrm{L}$ & U.S. & & & $<8$ & $<8$ & $<8$ & $<4$ & $<8$ & $<8$ & $<8$ & $<8$ \\
\hline Benzo(ghi)perylene & $\mu \mathrm{g} / \mathrm{L}$ & U.S. & & & $<10$ & $<10$ & $<10$ & $<5$ & $<10$ & $<10$ & $<10$ & $<10$ \\
\hline Benzo(k)fluoroanthene & $\mu \mathrm{g} / \mathrm{L}$ & U.S. & & & $<8$ & $<8$ & $<8$ & $<4$ & $<8$ & $<8$ & $<8$ & $<8$ \\
\hline Fluorene & $\mu \mathrm{g} / \mathrm{L}$ & U.S. & 1,000 & EPA DWEL & $<16$ & $<16$ & $<16$ & $<8$ & $<16$ & $<16$ & $<16$ & $<16$ \\
\hline Indeno(1,2,3-cd)pyrene & $\mu \mathrm{g} / \mathrm{L}$ & U.S. & & & $<10$ & $<10$ & $<10$ & $<5$ & $<10$ & $<10$ & $<10$ & $<10$ \\
\hline Naphthalene & $\mu \mathrm{g} / \mathrm{L}$ & U.S. & 100 & EPA HA & $\angle 40$ & $\angle 40$ & $\angle 40$ & $<20$ & $\angle 40$ & $<40$ & $\angle 40$ & $\angle 40$ \\
\hline Phenanthrene & $\mu \mathrm{g} / \mathrm{L}$ & U.S. & & & $<8$ & $<8$ & $<8$ & $<4$ & $<8$ & $<8$ & $<8$ & $<8$ \\
\hline Pyrene & $\mu \mathrm{g} / \mathrm{L}$ & U.S. & & & $<8$ & $<8$ & $<8$ & $<4$ & $<8$ & $<8$ & $<8$ & $<8$ \\
\hline bis(2-Chloroethyl) ether & $\mu \mathrm{g} / \mathrm{L}$ & U.S. & & & $<16$ & $<16$ & $<16$ & $<8$ & $<16$ & $<16$ & $<16$ & $<16$ \\
\hline
\end{tabular}

NA=Not analyzed;

SWEG - 1000 days (11-2008) 


\begin{tabular}{|c|c|c|c|c|c|c|c|c|c|c|c|c|}
\hline Mission & \multirow[b]{2}{*}{ Units } & \multirow[b]{2}{*}{$\begin{array}{c}\text { Test } \\
\begin{array}{c}\text { Conducted } \\
\text { by }\end{array} \\
\end{array}$} & \multirow[b]{2}{*}{$\begin{array}{c}\text { Potable Water } \\
\text { Maximum } \\
\text { Contaminant } \\
\text { Level } \\
\text { (MCL) } \\
\end{array}$} & \multirow[b]{2}{*}{$\begin{array}{c}\text { Maximum } \\
\text { Contaminant } \\
\text { Level } \\
\text { Source } \\
\end{array}$} & \multicolumn{2}{|c|}{ Soyuz 19/Exp. 21} & \multicolumn{2}{|c|}{ ISS ULF3/Exp. 21} & \multirow[b]{2}{*}{\begin{tabular}{|c|} 
ISS 20A/Exp. 22 \\
WPA PWD \\
Ambient \\
\\
Potable Water \\
$1 / 6 / 2010$ \\
20100222001 \\
\end{tabular}} & \multicolumn{2}{|c|}{ ISS 19A/Exp. 23} & \multirow[b]{2}{*}{\begin{tabular}{|c|} 
Soyuz 21/Exp. 23 \\
W PA PWD Hot \\
\\
\\
Potable Water \\
3/31/2010 \\
20100603003
\end{tabular}} \\
\hline $\begin{array}{l}\text { Sample Location } \\
\text { Sample Description } \\
\text { Sample Date } \\
\text { Analysis/Sample ID }\end{array}$ & & & & & \begin{tabular}{|c|} 
WPA PWD Hot \\
\\
Potable W ater \\
10/20/2009 \\
20091214001 \\
\end{tabular} & $\begin{array}{c}\text { WPA PWD } \\
\text { Ambient } \\
\text { Potable Water } \\
\text { 10/20/2009 } \\
20091214002\end{array}$ & \begin{tabular}{|c|} 
WPA PWD Hot \\
\\
Potable Water \\
$11 / 10 / 2009$ \\
20091130012 \\
\end{tabular} & $\begin{array}{c}\text { W PA PWD } \\
\text { Ambient } \\
\\
\text { Potable Water } \\
\text { 11/10/2009 } \\
20091130013\end{array}$ & & $\begin{array}{c}\text { WPA PWD } \\
\text { Ambient } \\
\\
\text { Potable Water } \\
3 / 3 / 2010 \\
20100422006\end{array}$ & \begin{tabular}{|c|} 
WPA PWD Hot \\
\\
Potable W ater \\
$3 / 3 / 2010$ \\
20100422007 \\
\end{tabular} & \\
\hline bis(2-Chloroethoxy) methane & $\mu \mathrm{g} / \mathrm{L}$ & U.S. & & & $<16$ & $<16$ & $<16$ & $<8$ & $<16$ & $<16$ & $<16$ & $<16$ \\
\hline bis(2-Chloroisopropyl) ether & $\mu \mathrm{g} / \mathrm{L}$ & U.S. & 300 & EPA HA & $<16$ & $<16$ & $<16$ & $<8$ & $<16$ & $<16$ & $<16$ & $<16$ \\
\hline 4-Bromophenyl phenyl ether & $\mu \mathrm{g} / \mathrm{L}$ & U.S. & & & $<16$ & $<16$ & $<16$ & $<8$ & $<16$ & $<16$ & $<16$ & $<16$ \\
\hline 4-Chlorophenyl phenyl ether & $\mu \mathrm{g} / \mathrm{L}$ & U.S. & & & $<16$ & $<16$ & $<16$ & $<8$ & $<16$ & $<16$ & $<16$ & $<16$ \\
\hline 1,2-Dichlorobenzene & $\mu \mathrm{g} / \mathrm{L}$ & U.S. & 600 & EPA & $<16$ & $<16$ & $<16$ & $<8$ & $<16$ & $<16$ & $<16$ & $<16$ \\
\hline 1,3-Dichlorobenzene & $\mu \mathrm{g} / \mathrm{L}$ & U.S. & 600 & EPA HA & $<16$ & $<16$ & $<16$ & $<8$ & $<16$ & $<16$ & $<16$ & $<16$ \\
\hline 1,4-Dichlorobenzene & $\mu \mathrm{g} / \mathrm{L}$ & U.S. & 75 & EPA & $<16$ & $<16$ & $<16$ & $<8$ & $<16$ & $<16$ & $<16$ & $<16$ \\
\hline \begin{tabular}{|l} 
Hexachlorobenzene \\
\end{tabular} & $\mu \mathrm{g} / \mathrm{L}$ & U.S. & 30 & EPA DWEL & $<16$ & $<16$ & $<16$ & $<8$ & $<16$ & $<16$ & $<16$ & $<16$ \\
\hline Hexachlorobutadiene & $\mu \mathrm{g} / \mathrm{L}$ & U.S. & 1 & EPA HA & $<16$ & $<16$ & $<16$ & $<8$ & $<16$ & $<16$ & $<16$ & $<16$ \\
\hline Hexachlorocyclopentadiene & $\mu \mathrm{g} / \mathrm{L}$ & U.S. & 50 & EPA & $<16$ & $<16$ & $<16$ & $<8$ & $<16$ & $<16$ & $<16$ & $<16$ \\
\hline Hexachloroethane & $\mu \mathrm{g} / \mathrm{L}$ & U.S. & 1 & EPA HA & $<16$ & $<16$ & $<16$ & $<8$ & $<16$ & $<16$ & $<16$ & $<16$ \\
\hline 1,2,4-Trichlorobenzene & $\mu \mathrm{g} / \mathrm{L}$ & U.S. & 70 & EPA & $<16$ & $<16$ & $<16$ & $<8$ & $<16$ & $<16$ & $<16$ & $<16$ \\
\hline \multicolumn{13}{|l|}{\begin{tabular}{|l|} 
Alcohols (DAI/GC/MS) \\
\end{tabular}} \\
\hline 1-Butanol & $\mu \mathrm{g} / \mathrm{L}$ & U.S. & & & $<100$ & $<100$ & $<100$ & $<100$ & $<100$ & $<100$ & $<100$ & $<100$ \\
\hline Ethanol & $\mu \mathrm{g} / \mathrm{L}$ & U.S. & & & $<100$ & $<100$ & $<100$ & $<100$ & $<100$ & $<100$ & $<100$ & $<100$ \\
\hline Methanol & $\mu \mathrm{g} / \mathrm{L}$ & U.S. & 40000 & SWEG & $<100$ & $<100$ & $<100$ & $<100$ & $<100$ & $<100$ & $<100$ & $<100$ \\
\hline 2-Methyl-1-butanol & $\mu \mathrm{g} / \mathrm{L}$ & U.S. & & & $<100$ & $<100$ & $<100$ & $<100$ & $<100$ & $<100$ & $<100$ & $<100$ \\
\hline 2-Methyl-2-butanol & $\mu \mathrm{g} / \mathrm{L}$ & U.S. & & & $<100$ & $<100$ & $<100$ & $<100$ & $<100$ & $<100$ & $<100$ & $<100$ \\
\hline 3-Methyl-1-butanol (Isopentanol) & $\mu \mathrm{g} / \mathrm{L}$ & U.S. & & & $<100$ & $<100$ & $<100$ & $<100$ & $<100$ & $<100$ & $<100$ & $<100$ \\
\hline 2-Methyl-1-propanol & $\mu \mathrm{g} / \mathrm{L}$ & U.S. & & & $<100$ & $<100$ & $<100$ & $<100$ & $<100$ & $<100$ & $<100$ & $<100$ \\
\hline 2-Methyl-2-propanol & $\mu \mathrm{g} / \mathrm{L}$ & U.S. & & & $<100$ & $<100$ & $<100$ & $<100$ & $<100$ & $<100$ & $<100$ & $<100$ \\
\hline 1-Pentanol (Amyl alcohol) & $\mu \mathrm{g} / \mathrm{L}$ & U.S. & & & $<100$ & $<100$ & $<100$ & $<100$ & $<100$ & $<100$ & $<100$ & $<100$ \\
\hline 2-Pentanol (sec-A myl alcohol) & $\mu \mathrm{g} / \mathrm{L}$ & U.S. & & & $<100$ & $<100$ & $<100$ & $<100$ & $<100$ & $<100$ & $<100$ & $<100$ \\
\hline 3-Pentanol & $\mu \mathrm{g} / \mathrm{L}$ & U.S. & & & $<100$ & $<100$ & $<100$ & $<100$ & $<100$ & $<100$ & $<100$ & $<100$ \\
\hline 1-Propanol & $\mu \mathrm{g} / \mathrm{L}$ & U.S. & & & $<100$ & $<100$ & $<100$ & $<100$ & $<100$ & $<100$ & $<100$ & $<100$ \\
\hline 2-Propanol (Isopropanol) & $\mu \mathrm{g} / \mathrm{L}$ & U.S. & & & $<100$ & $<100$ & $<100$ & $<100$ & $<100$ & $<100$ & $<100$ & $<100$ \\
\hline \multicolumn{13}{|l|}{ Glycols (DAI/GC/MS) } \\
\hline \begin{tabular}{|l|} 
1,2-Ethanediol (Ethylene glycol) \\
\end{tabular} & $\mu \mathrm{g} / \mathrm{L}$ & U.S. & $12000 / 4000$ & MORD/SWEG & $<1000$ & $<1000$ & $<1000$ & $<1000$ & $<1000$ & $<1000$ & $<1000$ & $<1000$ \\
\hline 1,2-Propanediol (Propylene glycol) & $\mu \mathrm{g} / \mathrm{L}$ & U.S. & 1700000 & SWEG & $<500$ & $<500$ & $<500$ & $<500$ & $<500$ & $<500$ & $<500$ & $<500$ \\
\hline \multicolumn{13}{|l|}{ Glycerol (LC/MS/MS) } \\
\hline Glycerol (1,2,3-Propanetriol) & $\mu \mathrm{g} / \mathrm{L}$ & U.S. & & & $\mathrm{NA}$ & $\mathrm{NA}$ & $\mathrm{NA}$ & $\mathrm{NA}$ & $\mathrm{NA}$ & $\mathrm{NA}$ & NA & $\mathrm{NA}$ \\
\hline & & & & & & & & & & & & \\
\hline \multicolumn{13}{|c|}{ Silanols (GC/MS \& LC/MS/MS) (R\&D Method - NIST traceable standard not available) } \\
\hline Dimethylsilanediol (DMSD) & $\mu \mathrm{g} / \mathrm{L}$ & U.S. & & & $\mathrm{NA}$ & NA & $\mathrm{NA}$ & $<400$ & $<400$ & $<400$ & $<400$ & $\mathrm{NA}$ \\
\hline Formate & $\mu \mathrm{g} / \mathrm{L}$ & U.S. & $2,500,000$ & SWEG & $<125$ & $<125$ & $<125$ & $<125$ & $<125$ & $<125$ & $<125$ & $<125$ \\
\hline Glycolate & $\mu \mathrm{g} / \mathrm{L}$ & U.S. & & & $<125$ & $<125$ & $<125$ & $<125$ & $<125$ & $<125$ & $<125$ & $<125$ \\
\hline Glyoxylate & $\mu \mathrm{g} / \mathrm{L}$ & U.S. & & & $<125$ & $<125$ & $<125$ & $<125$ & $<125$ & $<125$ & $<125$ & $<125$ \\
\hline Lactate & $\mu \mathrm{g} / \mathrm{L}$ & U.S. & & & $<1000$ & $<1000$ & $<1000$ & $<1000$ & $<1000$ & $<1000$ & $<1000$ & $<1000$ \\
\hline Oxalate & $\mu \mathrm{g} / \mathrm{L}$ & U.S. & & & $<125$ & $<125$ & $<125$ & $<125$ & $<125$ & $<125$ & $<125$ & $<125$ \\
\hline Propionate & $\mu \mathrm{g} / \mathrm{L}$ & U.S. & & & $<125$ & $<125$ & $<125$ & $<125$ & $<125$ & $<125$ & $<125$ & $<125$ \\
\hline
\end{tabular}

NA=Not analyzed;

SWEG - 1000 days (11-2008) 
Appendix 3. ISS WPA RIP and PWD Summary for Expeditions 21 through 25

\begin{tabular}{|c|c|c|c|c|c|c|c|c|c|c|c|c|}
\hline \multirow[b]{2}{*}{\begin{tabular}{|l} 
Mission \\
Sample Location \\
Sample Description \\
Sample Date \\
Analysis/Sample ID \\
\end{tabular}} & \multirow[b]{2}{*}{ Units } & \multirow[b]{2}{*}{$\begin{array}{c}\text { Test } \\
\text { Conducted } \\
\text { by } \\
\end{array}$} & \multirow[b]{2}{*}{$\begin{array}{c}\text { Potable Water } \\
\text { Maximum } \\
\text { Contaminant } \\
\text { Level } \\
\text { (MCL) } \\
\end{array}$} & \multirow[b]{2}{*}{$\begin{array}{c}\text { Maximum } \\
\text { Contaminant } \\
\text { Level } \\
\text { Source } \\
\end{array}$} & \multicolumn{2}{|c|}{ Soyuz 19/Exp. 21} & \multicolumn{2}{|c|}{ ISS ULF3/Exp. 21} & \multirow[b]{2}{*}{\begin{tabular}{|c|} 
ISS 20A/Exp. 22 \\
WPA PWD \\
Ambient \\
\\
Potable Water \\
$1 / 6 / 2010$ \\
20100222001 \\
\end{tabular}} & \multicolumn{2}{|c|}{ ISS 19A/Exp. 23} & \multirow[b]{2}{*}{\begin{tabular}{|c|} 
Soyuz 21/Exp. 23 \\
W PA PW D Hot \\
\\
\\
Potable Water \\
3/31/2010 \\
20100603003
\end{tabular}} \\
\hline & & & & & $\begin{array}{c}\text { W PA PW D Hot } \\
\text { Potable W ater } \\
\text { 10/20/2009 } \\
20091214001 \\
\end{array}$ & $\begin{array}{c}\text { WPA PWD } \\
\text { A mbient } \\
\text { Potable Water } \\
\text { 10/20/2009 } \\
20091214002 \\
\end{array}$ & \begin{tabular}{|c|} 
Potable Water \\
$11 / 10 / 2009$ \\
20091130012 \\
\end{tabular} & $\begin{array}{c}\text { Potable W ater } \\
11 / 10 / 2009 \\
20091130013 \\
\end{array}$ & & $\begin{array}{c}\text { WPA PWD } \\
\text { Ambient } \\
\\
\text { Potable Water } \\
3 / 3 / 2010 \\
20100422006 \\
\end{array}$ & \begin{tabular}{|c|} 
WPA PW D Hot \\
\\
Potable W ater \\
$3 / 3 / 2010$ \\
20100422007 \\
\end{tabular} & \\
\hline \multicolumn{13}{|l|}{ Aldehydes } \\
\hline Formaldehyde & $\mu \mathrm{g} / \mathrm{L}$ & U.S. & $12,000 / 1,000$ & SWEG/EPA HA & $<2$ & 2 & $<2$ & $<2$ & 4 & 5 & 2 & 4 \\
\hline Ethylamine & $\mu \mathrm{g} / \mathrm{L}$ & U.S. & \begin{tabular}{|l|} 
Monoalkylamines 2000 \\
\end{tabular} & SWEG & $<125$ & $<125$ & $<125$ & $<125$ & $<125$ & $<125$ & $<125$ & $<125$ \\
\hline Methylamine & $\mu \mathrm{g} / \mathrm{L}$ & U.S. & \begin{tabular}{|l|} 
Monoalkylamines 2000 \\
\end{tabular} & SWEG & $<125$ & $<125$ & $<125$ & $<125$ & $<125$ & $<125$ & $<125$ & $<125$ \\
\hline n-Propylamine & $\mu \mathrm{g} / \mathrm{L}$ & U.S. & \begin{tabular}{|l|} 
Monoalkylamines 2000 \\
\end{tabular} & SWEG & $<125$ & $<125$ & $<125$ & $<125$ & $<125$ & $<125$ & $<125$ & $<125$ \\
\hline Trimethylamine & $\mu \mathrm{g} / \mathrm{L}$ & U.S. & \begin{tabular}{|l|} 
Trialkylamines 400 \\
\end{tabular} & SWEG & $<125$ & $<125$ & $<125$ & $<125$ & $<125$ & $<125$ & $<125$ & $<125$ \\
\hline \multicolumn{13}{|l|}{ Non-volatiles (LC/UV-VIS) } \\
\hline Urea & $\mu \mathrm{g} / \mathrm{L}$ & U.S. & & & $<800$ & $<800$ & $<800$ & $<800$ & $<800$ & $<800$ & $<800$ & $<800$ \\
\hline Caprolactam & $\mu \mathrm{g} / \mathrm{L}$ & U.S. & 100,000 & SWEG & $<8$ & $<8$ & $<8$ & $<4$ & $<8$ & $<8$ & $<8$ & $<8$ \\
\hline Organic Carbon Recovery & percent & U.S. & & & 9.60 & 12.05 & 7.59 & 6.33 & 6.46 & 24.73 & 18.64 & 4.73 \\
\hline Unaccounted Organic Carbon & $\mathrm{mg} / \mathrm{L}$ & U.S. & & & 0.27 & 0.19 & 0.21 & 0.13 & 0.15 & 0.13 & 0.13 & 0.24 \\
\hline
\end{tabular}


Appendix 3. ISS WPA RIP and PWD Summary for Expeditions 21 through 25

\begin{tabular}{|c|c|c|c|c|c|c|c|c|c|c|c|}
\hline \multirow[b]{2}{*}{\begin{tabular}{|l} 
Mission \\
\\
Sample Location \\
Sample Description \\
Sample Date \\
Analysis/Sample ID \\
\end{tabular}} & \multirow[b]{2}{*}{ Units } & \multicolumn{2}{|c|}{ ISS ULF4/Exp. 23} & \multicolumn{3}{|c|}{ Soyuz 22/Exp. 24} & \multicolumn{2}{|c|}{ Soyuz 23/Exp. 25} & \multicolumn{2}{|c|}{ ISS 19A/Exp. 23} & \multirow[b]{2}{*}{$\begin{array}{c}\text { Soyuz 22/Exp. } 24 \\
\text { W PA RIP } \\
\\
\text { Processed Water } \\
7 / 29 / 2010 \\
20100926004 \\
\end{array}$} \\
\hline & & $\begin{array}{c}\text { WPA PWD } \\
\text { Ambient } \\
\\
\text { Potable Water } \\
4 / 26 / 2010 \\
20100527006\end{array}$ & $\begin{array}{l}\text { W PA PWD } \\
\text { Ambient } \\
\\
\text { Potable Water } \\
5 / 18 / 2010 \\
20100527007\end{array}$ & $\begin{array}{c}\text { WPA PWD } \\
\text { Ambient } \\
\\
\text { Potable Water } \\
7 / 14 / 2010 \\
20100926001\end{array}$ & \begin{tabular}{|c|} 
W PA PWD Hot \\
\\
Potable W ater \\
$8 / 25 / 2010$ \\
20100926002 \\
\end{tabular} & $\begin{array}{l}\text { WPA PWD } \\
\text { Ambient } \\
\\
\text { Potable Water } \\
9 / 15 / 2010 \\
20100926003 \\
\end{array}$ & \begin{tabular}{|c|} 
W PA PWD Hot \\
\\
Potable W ater \\
$10 / 19 / 2010$ \\
20101129001 \\
\end{tabular} & \begin{tabular}{|c|} 
WPA PWD Hot \\
\\
Potable Water \\
$11 / 23 / 2010$ \\
20101129002 \\
\end{tabular} & \begin{tabular}{c|} 
PWD Aux Port \\
\\
Processed \\
Water \\
$2 / 25 / 2010$ \\
20100422009 \\
\end{tabular} & \begin{tabular}{|c|} 
WPA RIP \\
\\
Processed \\
Water \\
$2 / 25 / 2010$ \\
20100422008 \\
\end{tabular} & \\
\hline \multicolumn{12}{|l|}{ Physical Characteristics } \\
\hline $\mathrm{pH}$ & $\mathrm{pH}$ units & 7.35 & 7.25 & 7.95 & 7.88 & 7.56 & 6.86 & 6.95 & 6.05 & 6.18 & 7.31 \\
\hline Conductivity & $\mu \mathrm{S} / \mathrm{cm}$ & 2 & 1 & 1 & 1 & 1 & 4 & 2 & 3 & 3 & 3 \\
\hline Turbidity & NTU & $<0.1$ & $<0.1$ & $<0.1$ & $<0.1$ & $<0.1$ & $\mathrm{NA}$ & $<0.1$ & 0.1 & $<0.1$ & $\mathrm{NA}$ \\
\hline Total Solids & $\mathrm{mg} / \mathrm{L}$ & $<5$ & $<5$ & $\mathrm{NA}$ & $\mathrm{NA}$ & $\mathrm{NA}$ & $\mathrm{NA}$ & $\mathrm{NA}$ & $<5$ & $<5$ & $\mathrm{NA}$ \\
\hline \multicolumn{12}{|l|}{ Iodine (LCV) } \\
\hline Total I & $\mathrm{mg} / \mathrm{L}$ & $<0.05$ & $<0.05$ & $<0.05$ & $<0.05$ & $<0.05$ & $<0.05$ & $<0.05$ & 2.24 & 2.72 & 2.63 \\
\hline Iodine & $\mathrm{mg} / \mathrm{L}$ & $<0.05$ & $<0.05$ & $<0.05$ & $<0.05$ & $<0.05$ & $<0.05$ & $<0.05$ & 1.11 & 2.03 & 2.05 \\
\hline Iodide & $\mathrm{mg} / \mathrm{L}$ & $<0.05$ & $<0.05$ & $<0.05$ & $<0.05$ & $<0.05$ & $<0.05$ & $<0.05$ & 1.13 & 0.69 & 0.58 \\
\hline \multicolumn{11}{|l|}{ Anions (IC/ISE) } & \\
\hline Bromide & $\mathrm{mg} / \mathrm{L}$ & $<0.5$ & $<0.5$ & $<0.5$ & $<0.5$ & $<0.5$ & $<0.5$ & $<0.5$ & $<0.5$ & $<0.5$ & $<0.5$ \\
\hline Chloride & $\mathrm{mg} / \mathrm{L}$ & $<0.15$ & $<0.15$ & $<0.15$ & $<0.15$ & $<0.15$ & $<0.15$ & $<0.15$ & $<0.15$ & $<0.15$ & $<0.15$ \\
\hline Fluoride & $\mathrm{mg} / \mathrm{L}$ & $<0.1$ & $<0.1$ & $<0.1$ & $<0.1$ & $<0.1$ & $<0.1$ & $<0.1$ & $<0.1$ & $<0.1$ & $<0.1$ \\
\hline Nitrate as Nitrogen (NO3-N) & $\mathrm{mg} / \mathrm{L}$ & $<0.11$ & $<0.11$ & $<0.11$ & $<0.11$ & $<0.11$ & $<0.11$ & $<0.11$ & $<0.11$ & $<0.11$ & $<0.11$ \\
\hline Nitrite as Nitrogen (NO2-N) & $\mathrm{mg} / \mathrm{L}$ & NA & NA & NA & NA & NA & NA & $\mathrm{NA}$ & $\mathrm{NA}$ & $\mathrm{NA}$ & NA \\
\hline \begin{tabular}{|l|} 
Phosphate as P (PO4-P) \\
\end{tabular} & $\mathrm{mg} / \mathrm{L}$ & $<0.24$ & $<0.24$ & $<0.02$ & $<0.02$ & $<0.02$ & $<0.01$ & $<0.01$ & $<0.24$ & $<0.24$ & $<0.02$ \\
\hline Sulfate & $\mathrm{mg} / \mathrm{L}$ & $<0.75$ & $<0.75$ & $<0.75$ & $<0.75$ & $<0.75$ & $<0.75$ & $<0.75$ & $<0.75$ & $<0.75$ & $<0.75$ \\
\hline & & \multicolumn{9}{|c|}{\begin{tabular}{|l|} 
Cations (IC) \\
\end{tabular}} & \\
\hline $\begin{array}{l}\text { Ammonia as Nitrogen (NH3-N) } \\
\end{array}$ & $\mathrm{mg} / \mathrm{L}$ & $<0.002$ & $<0.002$ & $<0.002$ & $<0.002$ & $<0.002$ & $<0.002$ & $<0.002$ & $<0.002$ & $<0.002$ & $<0.002$ \\
\hline Lithium & $\mathrm{mg} / \mathrm{L}$ & $<0.002$ & $<0.002$ & $<0.002$ & $<0.002$ & $<0.002$ & $<0.002$ & $<0.002$ & $<0.002$ & $<0.002$ & $<0.002$ \\
\hline \multicolumn{12}{|l|}{ Metals (ICP/MS) } \\
\hline \begin{tabular}{|l} 
Calcium \\
\end{tabular} & $\mathrm{mg} / \mathrm{L}$ & $<0.01$ & $<0.01$ & $<0.02$ & 0.03 & 0.03 & 0.03 & $<0.01$ & $<0.01$ & $<0.01$ & 0.03 \\
\hline \begin{tabular}{|l|} 
Magnesium \\
\end{tabular} & $\mathrm{mg} / \mathrm{L}$ & $<0.01$ & $<0.01$ & $<0.02$ & $<0.02$ & $<0.02$ & $<0.01$ & $<0.01$ & $<0.01$ & $<0.01$ & $<0.02$ \\
\hline Potassium & $\mathrm{mg} / \mathrm{L}$ & $<0.01$ & $<0.01$ & $<0.02$ & $<0.02$ & $<0.02$ & 0.03 & $<0.01$ & 0.01 & $<0.01$ & $<0.02$ \\
\hline \begin{tabular}{|l|} 
Sodium \\
\end{tabular} & $\mathrm{mg} / \mathrm{L}$ & $<0.01$ & 0.02 & $<0.02$ & $<0.02$ & $<0.02$ & 0.02 & $<0.01$ & $<0.01$ & $<0.01$ & $<0.02$ \\
\hline Aluminum & $\mu \mathrm{g} / \mathrm{L}$ & $<2$ & $<2$ & $<4$ & $<4$ & $<4$ & $<2$ & $<<2$ & $<2$ & $<2$ & $<4$ \\
\hline Antimony & $\mu \mathrm{g} / \mathrm{L}$ & $<2$ & $<2$ & $<4$ & $<4$ & $<4$ & $<2$ & $<2$ & $<2$ & $<2$ & $<4$ \\
\hline Arsenic & $\mu \mathrm{g} / \mathrm{L}$ & $<1$ & $<1$ & $<2$ & $<2$ & $<2$ & $<1$ & $<1$ & $<1$ & $<1$ & $<2$ \\
\hline Barium & $\mu \mathrm{g} / \mathrm{L}$ & $<1$ & $<1$ & $<2$ & $<2$ & 15 & $<1$ & $<1$ & $<1$ & $<1$ & $<2$ \\
\hline Beryllium & $\mu \mathrm{g} / \mathrm{L}$ & $<1$ & $<1$ & $<2$ & $<2$ & $<2$ & $<1$ & $<1$ & $<1$ & $<1$ & $<2$ \\
\hline Cadmium & $\mu \mathrm{g} / \mathrm{L}$ & $<1$ & $<1$ & $<2$ & $<2$ & $<2$ & $<1$ & $<1$ & $<1$ & $<1$ & $<2$ \\
\hline Chromium & $\mu \mathrm{g} / \mathrm{L}$ & $<5$ & $<5$ & $<10$ & $<10$ & $<10$ & $<5$ & $<5$ & $<5$ & $<5$ & $<10$ \\
\hline Cobalt & $\mu \mathrm{g} / \mathrm{L}$ & $<1$ & $<1$ & $<2$ & $<2$ & $<2$ & $<1$ & $<1$ & $<1$ & $<1$ & $<2$ \\
\hline Copper & $\mu \mathrm{g} / \mathrm{L}$ & $<1$ & $<1$ & $<2$ & $<2$ & $<2$ & $<1$ & $<1$ & $<1$ & $<1$ & $<2$ \\
\hline Iron & $\mu \mathrm{g} / \mathrm{L}$ & $<5$ & $<5$ & $<10$ & $<10$ & $<10$ & $<5$ & $<5$ & $<5$ & $<5$ & $<10$ \\
\hline Lead & $\mu \mathrm{g} / \mathrm{L}$ & $<1$ & $<1$ & $<2$ & $<2$ & $<2$ & $<1$ & $<1$ & $<1$ & $<1$ & $<2$ \\
\hline Manganese & $\mu \mathrm{g} / \mathrm{L}$ & $<1$ & $<1$ & $<2$ & $<2$ & $<2$ & $<1$ & $<1$ & $<1$ & $<1$ & $<2$ \\
\hline Mercury & $\mu \mathrm{g} / \mathrm{L}$ & $<0.5$ & $<0.5$ & $<1$ & $<1$ & $<1$ & $<0.5$ & $<0.5$ & $<0.5$ & $<0.5$ & $<1$ \\
\hline Molybdenum & $\mu \mathrm{g} / \mathrm{L}$ & $<1$ & $<1$ & $<2$ & $<2$ & $<2$ & $<1$ & $<1$ & $<1$ & $<1$ & $<2$ \\
\hline Nickel & $\mu \mathrm{g} / \mathrm{L}$ & 27 & 29 & 29 & 17 & 4 & 17 & 23 & 270 & 42 & 53 \\
\hline Selenium & $\mu \mathrm{g} / \mathrm{L}$ & $<1$ & $<1$ & $<2$ & $<2$ & $<2$ & $<1$ & $<1$ & $<1$ & $<1$ & $<2$ \\
\hline Silver & $\mu \mathrm{g} / \mathrm{L}$ & $<2$ & $<2$ & $<4$ & $<4$ & $<4$ & $<2$ & $<2$ & $<2$ & $<2$ & $<4$ \\
\hline
\end{tabular}


Appendix 3. ISS WPA RIP and PWD Summary for Expeditions 21 through 25

\begin{tabular}{|c|c|c|c|c|c|c|c|c|c|c|c|}
\hline \multirow[t]{2}{*}{ Mission } & \multirow[b]{3}{*}{ Units } & \multicolumn{2}{|c|}{ ISS ULF4/Exp. 23} & \multicolumn{3}{|c|}{ Soyuz 22/Exp. 24} & \multicolumn{2}{|c|}{ Soyuz 23/Exp. 25 } & \multicolumn{2}{|c|}{ ISS 19A/Exp. 23} & \multirow{3}{*}{\begin{tabular}{|c|} 
Soyuz 22/Exp. 24 \\
W PA RIP \\
\\
\\
Processed Water \\
$7 / 29 / 2010$ \\
20100926004 \\
\end{tabular}} \\
\hline & & $\begin{array}{l}\text { WPA PWD } \\
\text { Ambient }\end{array}$ & $\begin{array}{c}\text { WPA PWD } \\
\text { Ambient }\end{array}$ & $\begin{array}{c}\text { WPA PWD } \\
\text { Ambient }\end{array}$ & WPA PWD Hot & $\begin{array}{c}\text { WPA PWD } \\
\text { Ambient }\end{array}$ & WPA PWD Hot & W PA PW D Hot & PWD Aux Port & WPA RIP & \\
\hline $\begin{array}{l}\text { Sample Location } \\
\\
\text { Sample Description } \\
\text { Sample Date } \\
\text { Analysis/Sample ID }\end{array}$ & & $\begin{array}{c}\text { Potable Water } \\
4 / 26 / 2010 \\
20100527006\end{array}$ & $\begin{array}{c}\text { Potable W ater } \\
5 / 18 / 2010 \\
20100527007\end{array}$ & $\begin{array}{l}\text { Potable W ater } \\
7 / 14 / 2010 \\
20100926001\end{array}$ & \begin{tabular}{|c|} 
Potable W ater \\
$8 / 25 / 2010$ \\
20100926002 \\
\end{tabular} & $\begin{array}{c}\text { Potable Water } \\
9 / 15 / 2010 \\
20100926003\end{array}$ & \begin{tabular}{|c|} 
Potable W ater \\
$10 / 19 / 2010$ \\
20101129001 \\
\end{tabular} & \begin{tabular}{|c|} 
Potable Water \\
$11 / 23 / 2010$ \\
20101129002
\end{tabular} & \begin{tabular}{c|} 
Processed \\
Water \\
$2 / 25 / 2010$ \\
20100422009
\end{tabular} & $\begin{array}{c}\text { Processed } \\
\text { Water } \\
2 / 25 / 2010 \\
20100422008 \\
\end{array}$ & \\
\hline Zinc & $\mu \mathrm{g} / \mathrm{L}$ & $<1$ & $<1$ & $<2$ & \begin{tabular}{|c|}
$<2$ \\
\end{tabular} & 2 & \begin{tabular}{|l|}
$<1$ \\
\end{tabular} & \begin{tabular}{|c|}
2010 \\
\end{tabular} & \begin{tabular}{c|}
2 \\
\end{tabular} & $<1$ & \begin{tabular}{|c|}
$<2$ \\
\end{tabular} \\
\hline \multicolumn{12}{|l|}{ Silicon (ICP/MS) } \\
\hline Silicon (ICP/MS) & & $\mathrm{NA}$ & $\mathrm{NA}$ & 1530 & 2130 & 1210 & 2360 & 223 & NA & $\mathrm{NA}$ & 1770 \\
\hline \multicolumn{11}{|l|}{ Total Organic Carbon (Sievers) } & \\
\hline $\begin{array}{l}\text { Total Inorganic Carbon } \\
\end{array}$ & $\mathrm{mg} / \mathrm{L}$ & 0.87 & 0.84 & 1.17 & 0.93 & 1.05 & 0.98 & 0.73 & 1.21 & 1.31 & 0.94 \\
\hline Total Organic Carbon & $\mathrm{mg} / \mathrm{L}$ & 0.16 & 0.22 & 1.51 & 2.19 & 1.11 & 2.51 & 0.15 & 0.24 & 0.18 & 2.18 \\
\hline \multicolumn{12}{|l|}{ Volatile Organics } \\
\hline Acetone & $\mu \mathrm{g} / \mathrm{L}$ & 11 & $<2$ & $<2$ & $<2$ & $<2$ & 40 & $<2$ & 25 & $<2$ & $<6$ \\
\hline Acryloniltrile & $\mu \mathrm{g} / \mathrm{L}$ & $<2$ & $<2$ & $<2$ & $<2$ & $<2$ & $<6$ & $<2$ & $<2$ & $<2$ & $<6$ \\
\hline Allyl chloride (3-Chloropropene) & $\mu \mathrm{g} / \mathrm{L}$ & $<2$ & $<2$ & $<2$ & $<2$ & $<2$ & $<6$ & $<2$ & $<2$ & $<2$ & $<6$ \\
\hline Benzene & $\mu \mathrm{g} / \mathrm{L}$ & $<0.4$ & $<0.4$ & $<0.4$ & $<0.4$ & $<0.4$ & $<1.2$ & $<0.4$ & $<0.4$ & $<0.4$ & $<1.2$ \\
\hline Bromobenzene & $\mu \mathrm{g} / \mathrm{L}$ & $<0.4$ & $<0.4$ & $<0.4$ & $<0.4$ & $<0.4$ & $<1.2$ & $<0.4$ & $<0.4$ & $<0.4$ & $<1.2$ \\
\hline Bromochloromethane & $\mu \mathrm{g} / \mathrm{L}$ & $<4$ & $<4$ & $<4$ & $<4$ & $<4$ & $<12$ & $<4$ & $<4$ & $<4$ & $<12$ \\
\hline Bromodichloromethane & $\mu \mathrm{g} / \mathrm{L}$ & $<0.4$ & $<0.4$ & $<0.4$ & $<0.4$ & $<0.4$ & $<1.2$ & $<0.4$ & $<0.4$ & $<0.4$ & $<1.2$ \\
\hline Bromoform & $\mu \mathrm{g} / \mathrm{L}$ & $<2$ & $<2$ & $<2$ & $<2$ & $<2$ & $<6$ & $<2$ & $<2$ & $<2$ & $<6$ \\
\hline Bromomethane & $\mu \mathrm{g} / \mathrm{L}$ & $<2$ & $<2$ & $<2$ & $<2$ & $<2$ & $<6$ & $<2$ & $<2$ & $<2$ & $<6$ \\
\hline 2-Butanone (Methyl ethyl ketone) & $\mu \mathrm{g} / \mathrm{L}$ & $<2$ & $<2$ & $<2$ & 4 & 4 & 24 & $<2$ & $<2$ & $<2$ & $<6$ \\
\hline n-Butylbenzene & $\mu \mathrm{g} / \mathrm{L}$ & $<0.4$ & $<0.4$ & $<0.4$ & $<0.4$ & $<0.4$ & $<1.2$ & $<0.4$ & $<0.4$ & $<0.4$ & $<1.2$ \\
\hline Sec-Butylbenzene & $\mu \mathrm{g} / \mathrm{L}$ & $<0.4$ & $<0.4$ & $<0.4$ & $<0.4$ & $<0.4$ & $<1.2$ & $<0.4$ & $<0.4$ & $<0.4$ & $<1.2$ \\
\hline tert-Butylbenzene & $\mu \mathrm{g} / \mathrm{L}$ & $<0.4$ & $<0.4$ & $<0.4$ & $<0.4$ & $<0.4$ & $<1.2$ & $<0.4$ & $<0.4$ & $<0.4$ & $<1.2$ \\
\hline Carbon disulfide & $\mu \mathrm{g} / \mathrm{L}$ & $<2$ & $<2$ & $<2$ & $<2$ & $<2$ & $<6$ & $<2$ & $<2$ & $<2$ & $<6$ \\
\hline Carbon tetrachloride & $\mu \mathrm{g} / \mathrm{L}$ & $<0.4$ & $<0.4$ & $<0.4$ & $<0.4$ & $<0.4$ & $<1.2$ & $<0.4$ & $<0.4$ & $<0.4$ & $<1.2$ \\
\hline Chloroacetonitrile & $\mu \mathrm{g} / \mathrm{L}$ & $<10$ & $<10$ & $<10$ & $<10$ & $<10$ & $<30$ & $<10$ & $<10$ & $<10$ & $<30$ \\
\hline Chlorobenzene & $\mu \mathrm{g} / \mathrm{L}$ & $<0.4$ & $<0.4$ & $<0.4$ & $<0.4$ & $<0.4$ & $<1.2$ & $<0.4$ & $<0.4$ & $<0.4$ & $<1.2$ \\
\hline 1-Chlorobutane (Butyl chloride) & $\mu \mathrm{g} / \mathrm{L}$ & $<0.4$ & $<0.4$ & $<0.4$ & $<0.4$ & $<0.4$ & $<1.2$ & $<0.4$ & $<0.4$ & $<0.4$ & $<1.2$ \\
\hline Chloroethane & $\mu \mathrm{g} / \mathrm{L}$ & $<2$ & $<2$ & $<2$ & $<2$ & $<2$ & $<6$ & $<2$ & $<2$ & $<2$ & $<6$ \\
\hline \begin{tabular}{|l} 
Chloroform \\
\end{tabular} & $\mu \mathrm{g} / \mathrm{L}$ & $<0.4$ & $<0.4$ & $<0.4$ & $<0.4$ & $<0.4$ & $<1.2$ & $<0.4$ & $<0.4$ & $<0.4$ & $<1.2$ \\
\hline Chloromethane & $\mu \mathrm{g} / \mathrm{L}$ & $<2$ & $<2$ & $<2$ & $<2$ & $<2$ & $<6$ & $<2$ & $<2$ & $<2$ & $<6$ \\
\hline 2-Chlorotoluene & $\mu \mathrm{g} / \mathrm{L}$ & $<0.4$ & $<0.4$ & $<0.4$ & $<0.4$ & $<0.4$ & $<1.2$ & $<0.4$ & $<0.4$ & $<0.4$ & $<1.2$ \\
\hline 4-Chlorotoluene & $\mu \mathrm{g} / \mathrm{L}$ & $<0.4$ & $<0.4$ & $<0.4$ & $<0.4$ & $<0.4$ & $<1.2$ & $<0.4$ & $<0.4$ & $<0.4$ & $<1.2$ \\
\hline Dibromochloromethane & $\mu \mathrm{g} / \mathrm{L}$ & $<0.4$ & $<0.4$ & $<0.4$ & $<0.4$ & $<0.4$ & $<1.2$ & $<0.4$ & $<0.4$ & $<0.4$ & $<1.2$ \\
\hline 1,2-Dibromo-3-chloropropane (DBCP) & $\mu \mathrm{g} / \mathrm{L}$ & $<2$ & $<2$ & $<2$ & $<2$ & $<2$ & $<6$ & $<2$ & $<2$ & $<2$ & $<6$ \\
\hline 1,2-Dibromoethane (EDB) & $\mu \mathrm{g} / \mathrm{L}$ & $<0.4$ & $<0.4$ & $<0.4$ & $<0.4$ & $<0.4$ & $<1.2$ & $<0.4$ & $<0.4$ & $<0.4$ & $<1.2$ \\
\hline \begin{tabular}{|l|} 
Dibromomethane \\
\end{tabular} & $\mu \mathrm{g} / \mathrm{L}$ & $<0.4$ & $<0.4$ & $<0.4$ & $<0.4$ & $<0.4$ & $<1.2$ & $<0.4$ & $<0.4$ & $<0.4$ & $<1.2$ \\
\hline 1,2-Dichlorobenzene & $\mu \mathrm{g} / \mathrm{L}$ & $<0.4$ & $<0.4$ & $<0.4$ & $<0.4$ & $<0.4$ & $<1.2$ & $<0.4$ & $<0.4$ & $<0.4$ & $<1.2$ \\
\hline 1,3-Dichlorobenzene & $\mu \mathrm{g} / \mathrm{L}$ & $<0.4$ & $<0.4$ & $<0.4$ & $<0.4$ & $<0.4$ & $<1.2$ & $<0.4$ & $<0.4$ & $<0.4$ & $<1.2$ \\
\hline 1,4-Dichlorobenzene & $\mu \mathrm{g} / \mathrm{L}$ & $<0.4$ & $<0.4$ & $<0.4$ & $<0.4$ & $<0.4$ & $<1.2$ & $<0.4$ & $<0.4$ & $<0.4$ & $<1.2$ \\
\hline trans-1,4-Dichloro-2-butene & $\mu \mathrm{g} / \mathrm{L}$ & $<0.4$ & $<0.4$ & $<0.4$ & $<0.4$ & $<0.4$ & $<1.2$ & $<0.4$ & $<0.4$ & $<0.4$ & $<1.2$ \\
\hline \begin{tabular}{|l|} 
Dichlorodifluoromethane \\
\end{tabular} & $\mu \mathrm{g} / \mathrm{L}$ & $<2$ & $<2$ & $<2$ & $<2$ & $<2$ & $<6$ & $<2$ & $<2$ & $<2$ & $<6$ \\
\hline 1,1-Dichloroethane & $\mu \mathrm{g} / \mathrm{L}$ & $<0.4$ & $<0.4$ & $<0.4$ & $<0.4$ & $<0.4$ & $<1.2$ & $<0.4$ & $<0.4$ & $<0.4$ & $<1.2$ \\
\hline 1,2-Dichloroethane & $\mu \mathrm{g} / \mathrm{L}$ & $<0.4$ & $<0.4$ & $<0.4$ & $<0.4$ & $<0.4$ & $<1.2$ & $<0.4$ & $<0.4$ & $<0.4$ & $<1.2$ \\
\hline 1,1-Dichloroethene & $\mu \mathrm{g} / \mathrm{L}$ & $<0.4$ & $<0.4$ & $<0.4$ & $<0.4$ & $<0.4$ & $<1.2$ & $<0.4$ & $<0.4$ & $<0.4$ & $<1.2$ \\
\hline cis1,2-Dichloroethene & $\mu \mathrm{g} / \mathrm{L}$ & $<0.4$ & $<0.4$ & $<0.4$ & $<0.4$ & $<0.4$ & $<1.2$ & $<0.4$ & $<0.4$ & $<0.4$ & $<1.2$ \\
\hline \begin{tabular}{|l|} 
trans-1,2-Dichloroethene \\
\end{tabular} & $\mu \mathrm{g} / \mathrm{L}$ & $<0.4$ & $<0.4$ & $<0.4$ & $<0.4$ & $<0.4$ & $<1.2$ & $<0.4$ & $<0.4$ & $<0.4$ & $<1.2$ \\
\hline 1,2-Dichloropropane & $\mu \mathrm{g} / \mathrm{L}$ & $<0.4$ & $<0.4$ & $<0.4$ & $<0.4$ & $<0.4$ & $<1.2$ & $<0.4$ & $<0.4$ & $<0.4$ & $<1.2$ \\
\hline
\end{tabular}


Appendix 3. ISS WPA RIP and PWD Summary for Expeditions 21 through 25

\begin{tabular}{|c|c|c|c|c|c|c|c|c|c|c|c|}
\hline Mission & & ISS ULI & Exp. 23 & & Soyuz 22/Exp. 2 & & $\begin{array}{l}\text { Soyuz } 23 \\
\end{array}$ & 3/Exp. 25 & ISS 19A & Exp. 23 & \begin{tabular}{|l|} 
Soyuz 22/Exp. 24 \\
\end{tabular} \\
\hline & & $\begin{array}{l}\text { WPA PWD } \\
\text { Ambient }\end{array}$ & $\begin{array}{c}\text { WPA PWD } \\
\text { Ambient }\end{array}$ & $\begin{array}{c}\text { WPA PWD } \\
\text { Ambient }\end{array}$ & WPA PWD Hot & $\begin{array}{c}\text { WPA PWD } \\
\text { Ambient }\end{array}$ & WPA PWD Hot & WPA PWD Hot & PWD Aux Port & W PA RIP & W PA RIP \\
\hline $\begin{array}{l}\text { Sample Location } \\
\\
\text { Sample Description } \\
\text { Sample Date } \\
\text { Analysis/Sample ID }\end{array}$ & Units & $\begin{array}{c}\text { Potable Water } \\
4 / 26 / 2010 \\
20100527006\end{array}$ & $\begin{array}{c}\text { Potable W ater } \\
5 / 18 / 2010 \\
20100527007\end{array}$ & $\begin{array}{l}\text { Potable W ater } \\
7 / 14 / 2010 \\
20100926001\end{array}$ & \begin{tabular}{|c|} 
Potable W ater \\
$8 / 25 / 2010$ \\
20100926002 \\
\end{tabular} & $\begin{array}{c}\text { Potable Water } \\
9 / 15 / 2010 \\
20100926003\end{array}$ & \begin{tabular}{|c|} 
Potable W ater \\
$10 / 19 / 2010$ \\
20101129001 \\
\end{tabular} & \begin{tabular}{|c|} 
Potable Water \\
$11 / 23 / 2010$ \\
20101129002
\end{tabular} & \begin{tabular}{c|} 
Processed \\
Water \\
$2 / 25 / 2010$ \\
20100422009
\end{tabular} & $\begin{array}{c}\text { Processed } \\
\text { Water } \\
2 / 25 / 2010 \\
20100422008\end{array}$ & $\begin{array}{c}\text { Processed Water } \\
7 / 29 / 2010 \\
20100926004\end{array}$ \\
\hline 1,3-Dichloropropane & $\mu \mathrm{g} / \mathrm{L}$ & $<0.4$ & $<0.4$ & $<0.4$ & \begin{tabular}{l|}
$<0.4$ \\
\end{tabular} & $<0.4$ & \begin{tabular}{l|}
$<1.2$ \\
\end{tabular} & $<0.4$ & $<0.4$ & $<0.4$ & $<1.2$ \\
\hline 2,2-Dichloropropane & $\mu \mathrm{g} / \mathrm{L}$ & $<0.4$ & $<0.4$ & $<0.4$ & $<0.4$ & $<0.4$ & $<1.2$ & $<0.4$ & $<0.4$ & $<0.4$ & $<1.2$ \\
\hline 1,1-Dichloropropanone & $\mu \mathrm{g} / \mathrm{L}$ & $<2$ & $<2$ & $<2$ & $<2$ & $<2$ & $<6$ & $<2$ & $<2$ & $<2$ & $<6$ \\
\hline 1,1-Dichloropropene & $\mu \mathrm{g} / \mathrm{L}$ & $<0.4$ & $<0.4$ & $<0.4$ & $<0.4$ & $<0.4$ & $<1.2$ & $<0.4$ & $<0.4$ & $<0.4$ & $<1.2$ \\
\hline cis-1,3-Dichloropropene & $\mu \mathrm{g} / \mathrm{L}$ & $<0.4$ & $<0.4$ & $<0.4$ & $<0.4$ & $<0.4$ & $<1.2$ & $<0.4$ & $<0.4$ & $<0.4$ & $<1.2$ \\
\hline trans-1,3-Dichloropropene & $\mu \mathrm{g} / \mathrm{L}$ & $<2$ & $<2$ & $<2$ & $<2$ & $<2$ & $<6$ & $<2$ & $<2$ & $<2$ & $<6$ \\
\hline \begin{tabular}{|l|} 
Diethyl ether \\
\end{tabular} & $\mu \mathrm{g} / \mathrm{L}$ & $<2$ & $<2$ & $<2$ & $<2$ & $<2$ & $<6$ & $<2$ & $<2$ & $<2$ & $<6$ \\
\hline Ethylbenzene & $\mu \mathrm{g} / \mathrm{L}$ & $<0.4$ & $<0.4$ & $<0.4$ & $<0.4$ & $<0.4$ & $<1.2$ & $<0.4$ & $<0.4$ & $<0.4$ & $<1.2$ \\
\hline Ethyl methacrylate & $\mu \mathrm{g} / \mathrm{L}$ & $<2$ & $<2$ & $<2$ & $<2$ & $<2$ & $<6$ & $<2$ & $<2$ & $<2$ & $<6$ \\
\hline Hexachlorobutadiene & $\mu \mathrm{g} / \mathrm{L}$ & $<2$ & $<2$ & $<2$ & $<2$ & $<2$ & $<6$ & $<2$ & $<2$ & $<2$ & $<6$ \\
\hline Hexachloroethane & $\mu \mathrm{g} / \mathrm{L}$ & $<2$ & $<2$ & $<2$ & $<2$ & $<2$ & $<6$ & $<2$ & $<2$ & $<2$ & $<6$ \\
\hline 2-Hexanone & $\mu \mathrm{g} / \mathrm{L}$ & $<2$ & $<2$ & $<2$ & $<2$ & $<2$ & $<6$ & $<2$ & $<2$ & $<2$ & $<6$ \\
\hline Iodomethane & $\mu \mathrm{g} / \mathrm{L}$ & $<2$ & $<2$ & $<2$ & $<2$ & $<2$ & $<6$ & $<2$ & 5 & $<2$ & $<6$ \\
\hline Isopropylbenzene (Cumene) & $\mu \mathrm{g} / \mathrm{L}$ & $<0.4$ & $<0.4$ & $<0.4$ & $<0.4$ & $<0.4$ & $<1.2$ & $<0.4$ & $<0.4$ & $<0.4$ & $<1.2$ \\
\hline 4-Isopropyltoluene (Cymene) & $\mu \mathrm{g} / \mathrm{L}$ & $<0.4$ & $<0.4$ & $<0.4$ & $<0.4$ & $<0.4$ & $<1.2$ & $<0.4$ & $<0.4$ & $<0.4$ & $<1.2$ \\
\hline Methacrylonitrile & $\mu \mathrm{g} / \mathrm{L}$ & $<2$ & $<2$ & $<2$ & $<2$ & $<2$ & $<6$ & $<2$ & $<2$ & $<2$ & $<6$ \\
\hline Methyl acrylate & $\mu \mathrm{g} / \mathrm{L}$ & $<2$ & $<2$ & $<2$ & $<2$ & $<2$ & $<6$ & $<2$ & $<2$ & $<2$ & $<6$ \\
\hline Methyl-t-butylether (MTBE) & $\mu \mathrm{g} / \mathrm{L}$ & $<2$ & $<2$ & $<2$ & $<2$ & $<2$ & $<6$ & $<2$ & $<2$ & $<2$ & $<6$ \\
\hline \begin{tabular}{|l} 
Methylene chloride (Dichloromethane) \\
\end{tabular} & $\mu \mathrm{g} / \mathrm{L}$ & $<0.4$ & $<0.4$ & $<0.4$ & $<0.4$ & $<0.4$ & $<1.2$ & $<0.4$ & $<0.4$ & $<0.4$ & $<1.2$ \\
\hline Methyl methacrylate & $\mu \mathrm{g} / \mathrm{L}$ & $<2$ & $<2$ & $<2$ & $<2$ & $<2$ & $<6$ & $<2$ & $<2$ & $<2$ & $<6$ \\
\hline 4-Methyl-2-pentanone & $\mu \mathrm{g} / \mathrm{L}$ & $<0.4$ & $<0.4$ & $<0.4$ & $<0.4$ & $<0.4$ & $<1.2$ & $<0.4$ & $<0.4$ & $<0.4$ & $<1.2$ \\
\hline Naphthalene & $\mu \mathrm{g} / \mathrm{L}$ & $<0.4$ & $<0.4$ & $<0.4$ & $<0.4$ & $<0.4$ & $<1.2$ & $<0.4$ & $<0.4$ & $<0.4$ & $<1.2$ \\
\hline Nitrobenzene & $\mu \mathrm{g} / \mathrm{L}$ & $<2$ & $<2$ & $<2$ & $<2$ & $<2$ & $<6$ & $<2$ & $<2$ & $<2$ & $<6$ \\
\hline 2-Nitropropane & $\mu \mathrm{g} / \mathrm{L}$ & $<2$ & $<2$ & $<2$ & $<2$ & $<2$ & $<6$ & $<2$ & $<2$ & $<2$ & $<6$ \\
\hline \begin{tabular}{|l|} 
Pentachloroethane \\
\end{tabular} & $\mu \mathrm{g} / \mathrm{L}$ & $<2$ & $<2$ & $<2$ & $<2$ & $<2$ & $<6$ & $<2$ & $<2$ & $<2$ & $<6$ \\
\hline Propionitrile (Ethyl cyanide) & $\mu \mathrm{g} / \mathrm{L}$ & $<10$ & $<10$ & $<10$ & $<10$ & $<10$ & $<30$ & $<10$ & $<10$ & $<10$ & $<30$ \\
\hline n-Propylbenzene & $\mu \mathrm{g} / \mathrm{L}$ & $<0.4$ & $<0.4$ & $<0.4$ & $<0.4$ & $<0.4$ & $<1.2$ & $<0.4$ & $<0.4$ & $<0.4$ & $<1.2$ \\
\hline Styrene & $\mu \mathrm{g} / \mathrm{L}$ & $<0.4$ & $<0.4$ & $<0.4$ & $<0.4$ & $<0.4$ & $<1.2$ & $<0.4$ & $<0.4$ & $<0.4$ & $<1.2$ \\
\hline 1,1,1,2-Tetrachloroethane & $\mu \mathrm{g} / \mathrm{L}$ & $<0.4$ & $<0.4$ & $<0.4$ & $<0.4$ & $<0.4$ & $<1.2$ & $<0.4$ & $<0.4$ & $<0.4$ & $<1.2$ \\
\hline 1,1,2,2-Tetrachloroethane & $\mu \mathrm{g} / \mathrm{L}$ & $<0.4$ & $<0.4$ & $<0.4$ & $<0.4$ & $<0.4$ & $<1.2$ & $<0.4$ & $<0.4$ & $<0.4$ & $<1.2$ \\
\hline \begin{tabular}{|l|} 
Tetrachloroethene \\
\end{tabular} & $\mu \mathrm{g} / \mathrm{L}$ & $<0.4$ & $<0.4$ & $<0.4$ & $<0.4$ & $<0.4$ & $<1.2$ & $<0.4$ & $<0.4$ & $<0.4$ & $<1.2$ \\
\hline Tetrahydrofuran & $\mu \mathrm{g} / \mathrm{L}$ & $<2$ & $<2$ & $<2$ & $<2$ & $<2$ & $<6$ & $<2$ & $<2$ & $<2$ & $<6$ \\
\hline \begin{tabular}{|l|} 
Toluene \\
\end{tabular} & $\mu \mathrm{g} / \mathrm{L}$ & $<0.4$ & $<0.4$ & $<0.4$ & $<0.4$ & $<0.4$ & $<1.2$ & $<0.4$ & $<0.4$ & $<0.4$ & $<1.2$ \\
\hline 1,2,3-Trichlorobenzene & $\mu \mathrm{g} / \mathrm{L}$ & $<0.4$ & $<0.4$ & $<0.4$ & $<0.4$ & $<0.4$ & $<1.2$ & $<0.4$ & $<0.4$ & $<0.4$ & $<1.2$ \\
\hline 1,2,4-Trichlorobenzene & $\mu \mathrm{g} / \mathrm{L}$ & $<0.4$ & $<0.4$ & $<0.4$ & $<0.4$ & $<0.4$ & $<1.2$ & $<0.4$ & $<0.4$ & $<0.4$ & $<1.2$ \\
\hline 1,1,1-Trichloroethane & $\mu \mathrm{g} / \mathrm{L}$ & $<0.4$ & $<0.4$ & $<0.4$ & $<0.4$ & $<0.4$ & $<1.2$ & $<0.4$ & $<0.4$ & $<0.4$ & $<1.2$ \\
\hline 1,1,2-Trichloroethane & $\mu \mathrm{g} / \mathrm{L}$ & $<0.4$ & $<0.4$ & $<0.4$ & $<0.4$ & $<0.4$ & $<1.2$ & $<0.4$ & $<0.4$ & $<0.4$ & $<1.2$ \\
\hline Trichloroethene & $\mu \mathrm{g} / \mathrm{L}$ & $<0.4$ & $<0.4$ & $<0.4$ & $<0.4$ & $<0.4$ & $<1.2$ & $<0.4$ & $<0.4$ & $<0.4$ & $<1.2$ \\
\hline Trichlorofluoromethane & $\mu \mathrm{g} / \mathrm{L}$ & $<0.4$ & $<0.4$ & $<0.4$ & $<0.4$ & $<0.4$ & $<1.2$ & $<0.4$ & $<0.4$ & $<0.4$ & $<1.2$ \\
\hline 1,2,3-Trichloropropane & $\mu \mathrm{g} / \mathrm{L}$ & $<0.4$ & $<0.4$ & $<0.4$ & $<0.4$ & $<0.4$ & $<1.2$ & $<0.4$ & $<0.4$ & $<0.4$ & $<1.2$ \\
\hline 1,2,4-Trimethylbenzene & $\mu \mathrm{g} / \mathrm{L}$ & $<0.4$ & $<0.4$ & $<0.4$ & $<0.4$ & $<0.4$ & $<1.2$ & $<0.4$ & $<0.4$ & $<0.4$ & $<1.2$ \\
\hline 1,3,5-Trimethylbenzene & $\mu \mathrm{g} / \mathrm{L}$ & $<0.4$ & $<0.4$ & $<0.4$ & $<0.4$ & $<0.4$ & $<1.2$ & $<0.4$ & $<0.4$ & $<0.4$ & $<1.2$ \\
\hline \begin{tabular}{|l|l} 
Vinyl Acetate \\
\end{tabular} & $\mu \mathrm{g} / \mathrm{L}$ & $<2$ & $<2$ & $<2$ & $<2$ & $<2$ & $<6$ & $<2$ & $<2$ & $<2$ & $<6$ \\
\hline \begin{tabular}{|l|} 
Vinyl Chloride \\
\end{tabular} & $\mu \mathrm{g} / \mathrm{L}$ & $<2$ & $<2$ & $<2$ & $<2$ & $<2$ & $<6$ & $<2$ & $<2$ & $<2$ & $<6$ \\
\hline m\&p-Xylene & $\mu \mathrm{g} / \mathrm{L}$ & $<0.4$ & $<0.4$ & $<0.4$ & $<0.4$ & $<0.4$ & $<1.2$ & $<0.4$ & $<0.4$ & $<0.4$ & $<1.2$ \\
\hline 0 -Xylene & $\mu \mathrm{g} / \mathrm{L}$ & $<0.4$ & $<0.4$ & $<0.4$ & $<0.4$ & $<0.4$ & $<1.2$ & $<0.4$ & $<0.4$ & 1.5 & $<1.2$ \\
\hline & & & & & & & & & & & \\
\hline
\end{tabular}


Appendix 3. ISS WPA RIP and PWD Summary for Expeditions 21 through 25

\begin{tabular}{|c|c|c|c|c|c|c|c|c|c|c|c|}
\hline \multirow[t]{2}{*}{ Mission } & \multirow[b]{3}{*}{ Units } & \multicolumn{2}{|c|}{ ISS ULF4/Exp. 23} & \multicolumn{3}{|c|}{ Soyuz 22/Exp. 24} & \multicolumn{2}{|c|}{ Soyuz 23/Exp. 25} & \multicolumn{2}{|c|}{ ISS 19A/Exp. 23} & \multirow{3}{*}{\begin{tabular}{|c|} 
Soyuz 22/Exp. 24 \\
W PA RIP \\
\\
\\
Processed Water \\
$7 / 29 / 2010$ \\
20100926004 \\
\end{tabular}} \\
\hline & & $\begin{array}{c}\text { WPA PWD } \\
\text { Ambient }\end{array}$ & $\begin{array}{c}\text { WPA PWD } \\
\text { Ambient }\end{array}$ & $\begin{array}{c}\text { WPA PWD } \\
\text { Ambient }\end{array}$ & WPA PWD Hot & $\begin{array}{c}\text { WPA PWD } \\
\text { Ambient }\end{array}$ & WPA PWD Hot & WPA PW D Hot & PWD Aux Port & WPA RIP & \\
\hline $\begin{array}{l}\text { Sample Location } \\
\text { Sample Description } \\
\text { Sample Date } \\
\text { Analysis/Sample ID }\end{array}$ & & $\begin{array}{c}\text { Potable W ater } \\
4 / 26 / 2010 \\
20100527006\end{array}$ & $\begin{array}{c}\text { Potable W ater } \\
5 / 18 / 2010 \\
20100527007\end{array}$ & $\begin{array}{l}\text { Potable W ater } \\
7 / 14 / 2010 \\
20100926001\end{array}$ & \begin{tabular}{|c|} 
Potable W ater \\
$8 / 25 / 2010$ \\
20100926002 \\
\end{tabular} & $\begin{array}{c}\text { Potable Water } \\
9 / 15 / 2010 \\
20100926003\end{array}$ & \begin{tabular}{|c|} 
Potable W ater \\
$10 / 19 / 2010$ \\
20101129001 \\
\end{tabular} & \begin{tabular}{|c|} 
Potable Water \\
$11 / 23 / 2010$ \\
20101129002
\end{tabular} & \begin{tabular}{c|} 
Processed \\
Water \\
$2 / 25 / 2010$ \\
20100422009
\end{tabular} & $\begin{array}{c}\text { Processed } \\
\text { Water } \\
2 / 25 / 2010 \\
20100422008 \\
\end{array}$ & \\
\hline \multicolumn{12}{|c|}{ Volatile Organics - Non-Targets (Tentatively Identified Comp } \\
\hline \begin{tabular}{|l|} 
Acetaldehyde \\
\end{tabular} & $\mu \mathrm{g} / \mathrm{L}$ & not found & not found & not found & not found & not found & not found & not found & not found & not found & not found \\
\hline Butyraldehyde (Butanal) & $\mu \mathrm{g} / \mathrm{L}$ & not found & not found & not found & not found & not found & not found & not found & not found & not found & not found \\
\hline 1,8-Cineole & $\mu \mathrm{g} / \mathrm{L}$ & not found & not found & not found & not found & not found & not found & not found & not found & not found & not found \\
\hline Cyclohexanone & $\mu \mathrm{g} / \mathrm{L}$ & not found & not found & not found & not found & not found & not found & not found & not found & not found & not found \\
\hline Difluorodimethylsilane & $\mu \mathrm{g} / \mathrm{L}$ & not found & not found & not found & not found & not found & not found & not found & not found & not found & not found \\
\hline 2,5-Dimethylfuran & $\mu \mathrm{g} / \mathrm{L}$ & not found & not found & not found & not found & not found & not found & not found & not found & not found & not found \\
\hline 2,6-Dimethyl-1,7-octadiene & $\mu \mathrm{g} / \mathrm{L}$ & not found & not found & not found & not found & not found & not found & not found & not found & not found & not found \\
\hline Dimethyl sulfide (Thiobismethane) & $\mu \mathrm{g} / \mathrm{L}$ & not found & not found & not found & not found & not found & not found & not found & not found & not found & not found \\
\hline p-Dioxane & $\mu \mathrm{g} / \mathrm{L}$ & not found & not found & not found & not found & not found & not found & not found & not found & not found & not found \\
\hline 1,3-Dioxolane (Ethylene glycol formal) & $\mu \mathrm{g} / \mathrm{L}$ & not found & not found & not found & not found & not found & not found & not found & not found & not found & not found \\
\hline Ethyl acetate & $\mu \mathrm{g} / \mathrm{L}$ & not found & not found & not found & not found & not found & not found & not found & not found & not found & not found \\
\hline Fluorotrimethylsilane & $\mu \mathrm{g} / \mathrm{L}$ & not found & not found & not found & not found & not found & not found & not found & not found & not found & not found \\
\hline 2-Heptanone & $\mu \mathrm{g} / \mathrm{L}$ & not found & not found & not found & not found & not found & not found & not found & not found & not found & not found \\
\hline 4-Heptanone & $\mu \mathrm{g} / \mathrm{L}$ & not found & not found & not found & not found & not found & not found & not found & not found & not found & not found \\
\hline 3-Hexanone & $\mu \mathrm{g} / \mathrm{L}$ & not found & not found & not found & not found & not found & not found & not found & not found & not found & not found \\
\hline 3-Hydroxy-3-methylbutyric acid & $\mu \mathrm{g} / \mathrm{L}$ & not found & not found & not found & not found & not found & not found & not found & not found & not found & not found \\
\hline Isobutyronitrile & $\mu \mathrm{g} / \mathrm{L}$ & not found & not found & not found & not found & not found & not found & not found & not found & not found & not found \\
\hline Isooctanol & $\mu \mathrm{g} / \mathrm{L}$ & not found & not found & not found & not found & not found & not found & not found & not found & not found & not found \\
\hline L-Menthol & $\mu \mathrm{g} / \mathrm{L}$ & not found & not found & not found & not found & not found & not found & not found & not found & not found & not found \\
\hline Menthone & $\mu \mathrm{g} / \mathrm{L}$ & not found & not found & not found & not found & not found & not found & not found & not found & not found & not found \\
\hline Methyl acetate & $\mu \mathrm{g} / \mathrm{L}$ & not found & not found & not found & not found & not found & not found & not found & not found & not found & not found \\
\hline \begin{tabular}{|c|} 
cis-1-Methyl-4-(1-methylethenyl)-cyclohexane \\
\end{tabular} & $\mu \mathrm{g} / \mathrm{L}$ & not found & not found & not found & not found & not found & not found & not found & not found & not found & not found \\
\hline 3-Methyl-2-pentanone & $\mu \mathrm{g} / \mathrm{L}$ & not found & not found & not found & not found & not found & not found & not found & not found & not found & not found \\
\hline 2-Methyl-1-propene & $\mu \mathrm{g} / \mathrm{L}$ & not found & not found & not found & not found & not found & not found & not found & not found & not found & not found \\
\hline alpha-Methyl styrene & $\mu \mathrm{g} / \mathrm{L}$ & not found & not found & not found & not found & not found & not found & not found & not found & not found & not found \\
\hline 2-Nonanone & $\mu \mathrm{g} / \mathrm{L}$ & not found & not found & not found & not found & not found & not found & not found & not found & not found & not found \\
\hline 2-Octanone & $\mu \mathrm{g} / \mathrm{L}$ & not found & not found & not found & not found & not found & not found & not found & not found & not found & not found \\
\hline 2-Pentanone & $\mu \mathrm{g} / \mathrm{L}$ & not found & not found & not found & not found & not found & not found & not found & not found & not found & not found \\
\hline 1,1,2-Trichloro-1,2,2-trifluoroethane (Freon 113) & $\mu \mathrm{g} / \mathrm{L}$ & not found & not found & not found & not found & not found & not found & not found & not found & not found & not found \\
\hline Trimethylsilanol & $\mu \mathrm{g} / \mathrm{L}$ & not found & not found & not found & not found & not found & not found & not found & not found & not found & not found \\
\hline \multicolumn{12}{|l|}{\begin{tabular}{|l} 
Extractable Organics \\
\end{tabular}} \\
\hline Acetophenone & $\mu \mathrm{g} / \mathrm{L}$ & $<16$ & $<8$ & $<8$ & $<8$ & $<16$ & $<16$ & $<16$ & $<16$ & $<16$ & $<40$ \\
\hline Benzaldehyde & $\mu \mathrm{g} / \mathrm{L}$ & $<8$ & $<4$ & $<4$ & $<4$ & $<8$ & $<8$ & $<8$ & $<8$ & $<8$ & $<20$ \\
\hline \begin{tabular}{|l|} 
Benzoic acid \\
\end{tabular} & $\mu \mathrm{g} / \mathrm{L}$ & $<24$ & $<12$ & $<12$ & $<12$ & $<24$ & $\angle 48$ & $\angle 48$ & $<24$ & $<24$ & $<60$ \\
\hline Benzothiazole & $\mu \mathrm{g} / \mathrm{L}$ & $<8$ & $<4$ & $<4$ & $<4$ & $<8$ & $<8$ & $<8$ & $<8$ & $<8$ & $<20$ \\
\hline Benzyl alcohol & $\mu \mathrm{g} / \mathrm{L}$ & $<8$ & $<4$ & $<4$ & $<4$ & $<8$ & $<8$ & $<8$ & $<8$ & $<8$ & $<20$ \\
\hline Benzyl butyl phthlate & $\mu \mathrm{g} / \mathrm{L}$ & $<8$ & $<4$ & $<4$ & $<4$ & $<8$ & $<8$ & $<8$ & $<8$ & $<8$ & $<20$ \\
\hline 2-Butoxyethanol & $\mu \mathrm{g} / \mathrm{L}$ & $<16$ & $<8$ & $<8$ & $<8$ & $<16$ & $<16$ & $<16$ & $<16$ & $<16$ & $\angle 40$ \\
\hline 2-(2-Butoxyethoxy)ethanol & $\mu \mathrm{g} / \mathrm{L}$ & $<16$ & $<8$ & $<8$ & $<8$ & $<16$ & $<16$ & $<16$ & $<16$ & $<16$ & $\angle 40$ \\
\hline 2-(2-Butoxyethoxy)ethyl acetate & $\mu \mathrm{g} / \mathrm{L}$ & $<8$ & $<4$ & $<4$ & $<4$ & $<8$ & $<8$ & $<8$ & $<8$ & $<8$ & $<20$ \\
\hline n-Butylpalmitate & $\mu \mathrm{g} / \mathrm{L}$ & $<16$ & $<8$ & $<8$ & $<8$ & $<16$ & $<16$ & $<16$ & $<16$ & $<16$ & $<40$ \\
\hline Butylated hydroxyanisole (BHA) & $\mu \mathrm{g} / \mathrm{L}$ & $<8$ & $<4$ & $<4$ & $<4$ & $<8$ & $<8$ & $<8$ & $<8$ & $<8$ & $<20$ \\
\hline N-Butylbenzenesulfonamide & $\mu \mathrm{g} / \mathrm{L}$ & $<8$ & $<4$ & $<4$ & $<4$ & $<8$ & $<8$ & $<8$ & $<8$ & $<8$ & $<20$ \\
\hline 3-tert-Butylphenol & $\mu \mathrm{g} / \mathrm{L}$ & $<24$ & $<12$ & $<12$ & $<12$ & $<24$ & $<24$ & $<24$ & $<24$ & $<24$ & $<60$ \\
\hline Caffeine & $\mu \mathrm{g} / \mathrm{L}$ & $<8$ & $<4$ & $<4$ & $<4$ & $<8$ & $<8$ & $<8$ & $<8$ & $<8$ & $<20$ \\
\hline
\end{tabular}


Appendix 3. ISS WPA RIP and PWD Summary for Expeditions 21 through 25

\begin{tabular}{|c|c|c|c|c|c|c|c|c|c|c|c|}
\hline Mission & & ISS ULI & Exp. 23 & & Soyuz 22/Exp. 2 & & $\begin{array}{ll}\text { Soyuz } 23 \\
\end{array}$ & 3/Exp. 25 & ISS 19A & Exp. 23 & \begin{tabular}{|l|} 
Soyuz 22/Exp. 24 \\
\end{tabular} \\
\hline & & $\begin{array}{l}\text { WPA PWD } \\
\text { Ambient }\end{array}$ & $\begin{array}{c}\text { WPA PWD } \\
\text { Ambient }\end{array}$ & $\begin{array}{c}\text { WPA PWD } \\
\text { Ambient }\end{array}$ & WPA PWD Hot & $\begin{array}{c}\text { WPA PWD } \\
\text { A mbient }\end{array}$ & WPA PWD Hot & WPA PWD Hot & PWD Aux Port & WPA RIP & W PA RIP \\
\hline $\begin{array}{l}\text { Sample Location } \\
\\
\text { Sample Description } \\
\text { Sample Date } \\
\text { Analysis/Sample ID }\end{array}$ & Units & $\begin{array}{c}\text { Potable Water } \\
4 / 26 / 2010 \\
20100527006\end{array}$ & $\begin{array}{c}\text { Potable W ater } \\
5 / 18 / 2010 \\
20100527007\end{array}$ & $\begin{array}{l}\text { Potable W ater } \\
7 / 14 / 2010 \\
20100926001\end{array}$ & \begin{tabular}{|c|} 
Potable $W$ ater \\
$8 / 25 / 2010$ \\
20100926002 \\
\end{tabular} & $\begin{array}{l}\text { Potable Water } \\
9 / 15 / 2010 \\
20100926003\end{array}$ & \begin{tabular}{|c|} 
Potable W ater \\
$10 / 19 / 2010$ \\
20101129001 \\
\end{tabular} & $\begin{array}{c}\text { Potable Water } \\
11 / 23 / 2010 \\
20101129002\end{array}$ & \begin{tabular}{c|} 
Processed \\
Water \\
$2 / 25 / 2010$ \\
20100422009
\end{tabular} & \begin{tabular}{c|} 
Processed \\
Water \\
$2 / 25 / 2010$ \\
20100422008
\end{tabular} & $\begin{array}{c}\text { Processed Water } \\
7 / 29 / 2010 \\
20100926004\end{array}$ \\
\hline tris-2-Chloroethyl phosphate & $\mu \mathrm{g} / \mathrm{L}$ & $<8$ & $<4$ & $<4$ & $<4$ & $<8$ & $<8$ & $<8$ & $<8$ & $<8$ & $<20$ \\
\hline \begin{tabular}{|l|} 
Cholesterol \\
\end{tabular} & $\mu \mathrm{g} / \mathrm{L}$ & $<64$ & $<32$ & $<32$ & $<32$ & $<64$ & $<64$ & $<64$ & $<64$ & $<64$ & $<160$ \\
\hline o-Cresol (2-Methylphenol) & $\mu \mathrm{g} / \mathrm{L}$ & $<8$ & $<4$ & $<4$ & $<4$ & $<8$ & $<8$ & $<8$ & $<8$ & $<8$ & $<20$ \\
\hline Cyclododecane & $\mu \mathrm{g} / \mathrm{L}$ & $<8$ & $<4$ & $<4$ & $<4$ & $<8$ & $<8$ & $<8$ & $<8$ & $<8$ & $<20$ \\
\hline Decamethylcyclopentasiloxane & $\mu \mathrm{g} / \mathrm{L}$ & $<8$ & $<4$ & $<4$ & $<4$ & $<8$ & $<8$ & $<8$ & $<8$ & $<8$ & $<20$ \\
\hline \begin{tabular}{|l|} 
Decanoic acid \\
\end{tabular} & $\mu \mathrm{g} / \mathrm{L}$ & $<16$ & $<8$ & $<8$ & $<8$ & $<16$ & $<24$ & $<24$ & $<16$ & $<16$ & $\angle 40$ \\
\hline 2,6-Di-t-butyl-1,4-benzoquinone & $\mu \mathrm{g} / \mathrm{L}$ & $<8$ & $<4$ & $<4$ & $<4$ & $<8$ & $<8$ & $<8$ & $<8$ & $<8$ & $<20$ \\
\hline 2,4-Di-t-butylphenol & $\mu \mathrm{g} / \mathrm{L}$ & $<8$ & $<4$ & $<4$ & $<4$ & $<8$ & $<8$ & $<8$ & $<8$ & $<8$ & $<20$ \\
\hline 1,4 Diacetylbenzene & $\mu \mathrm{g} / \mathrm{L}$ & $<8$ & $<4$ & $<4$ & $<4$ & $<8$ & $<8$ & $<8$ & $<8$ & $<8$ & $<20$ \\
\hline N,N-Dibutylformamide & $\mu \mathrm{g} / \mathrm{L}$ & $<8$ & $<4$ & $<4$ & $<4$ & $<8$ & $<8$ & $<8$ & $<8$ & $<8$ & $<20$ \\
\hline \begin{tabular}{|l|} 
Dibutyl phthalate \\
\end{tabular} & $\mu \mathrm{g} / \mathrm{L}$ & $<8$ & $<4$ & $<4$ & $<4$ & $<8$ & $<8$ & $<8$ & $<8$ & $<8$ & $<20$ \\
\hline Dibutylamine & $\mu \mathrm{g} / \mathrm{L}$ & $<8$ & $<4$ & $<4$ & $<4$ & $<8$ & $<8$ & $<8$ & $<8$ & $<8$ & $<20$ \\
\hline N,N-Diethyl-m-toluamide & $\mu \mathrm{g} / \mathrm{L}$ & $<8$ & $<4$ & $<4$ & $<4$ & $<8$ & $<8$ & $<8$ & $<8$ & $<8$ & $<20$ \\
\hline Diethylphthalate & $\mu \mathrm{g} / \mathrm{L}$ & $<8$ & $<4$ & $<4$ & $<4$ & $<8$ & $<8$ & $<8$ & $<8$ & $<8$ & $<20$ \\
\hline Diethylene glycol monoethyl ether & $\mu \mathrm{g} / \mathrm{L}$ & $<8$ & $<4$ & $<4$ & $<4$ & $<8$ & $<8$ & $<8$ & $<8$ & $<8$ & $<20$ \\
\hline N,N-Diethylformamide & $\mu \mathrm{g} / \mathrm{L}$ & $<24$ & $<12$ & $<12$ & $<12$ & $<24$ & $<24$ & $<24$ & $<24$ & $<24$ & $<60$ \\
\hline Diiodomethane (Methyl iodide) & $\mu \mathrm{g} / \mathrm{L}$ & $<8$ & $<4$ & $<4$ & $<4$ & $<8$ & $<8$ & $<8$ & $<8$ & $<8$ & $<20$ \\
\hline Diisopropyl adipate & $\mu \mathrm{g} / \mathrm{L}$ & $<8$ & $<4$ & $<4$ & $<4$ & $<8$ & $<8$ & $<8$ & $<8$ & $<8$ & $<20$ \\
\hline Dimethyl phthalate & $\mu \mathrm{g} / \mathrm{L}$ & $<8$ & $<4$ & $<4$ & $<4$ & $<8$ & $<8$ & $<8$ & $<8$ & $<8$ & $<20$ \\
\hline N,N-Dimethyl acetamide & $\mu \mathrm{g} / \mathrm{L}$ & $<8$ & $<4$ & $<4$ & $<4$ & $<8$ & $<8$ & $<8$ & $<8$ & $<8$ & $<20$ \\
\hline N,N-Dimethylbenzylamine & $\mu \mathrm{g} / \mathrm{L}$ & $<8$ & $<4$ & $<4$ & $<4$ & $<8$ & $<8$ & $<8$ & $<8$ & $<8$ & $<20$ \\
\hline N,N-Dimethylformamide & $\mu \mathrm{g} / \mathrm{L}$ & $<16$ & $<8$ & $<8$ & $<8$ & $<16$ & $<16$ & $<16$ & $<16$ & $<16$ & $\angle 40$ \\
\hline Dipropylene glycol methyl ether & $\mu \mathrm{g} / \mathrm{L}$ & $<8$ & $<4$ & $<4$ & $<4$ & $<8$ & $<8$ & $<8$ & $<8$ & $<8$ & $<20$ \\
\hline \begin{tabular}{|l|} 
Dodecamethylcyclohexasiloxane \\
\end{tabular} & $\mu \mathrm{g} / \mathrm{L}$ & $<8$ & $<4$ & $<4$ & $<4$ & $<8$ & $<8$ & $<8$ & $<8$ & $<8$ & $<20$ \\
\hline 2-Ethoxyethanol & $\mu \mathrm{g} / \mathrm{L}$ & $<8$ & $<4$ & $<4$ & $<4$ & $<8$ & $<16$ & $<16$ & $<8$ & $<8$ & $<20$ \\
\hline 2-Ethyl-1-hexanol & $\mu \mathrm{g} / \mathrm{L}$ & $<8$ & $<4$ & $<4$ & $<4$ & $<8$ & $<8$ & $<8$ & $<8$ & $<8$ & $<20$ \\
\hline 2-Ethylhexanoic acid & $\mu \mathrm{g} / \mathrm{L}$ & $<8$ & $<4$ & $<4$ & $<4$ & $<8$ & $<16$ & $<16$ & $<8$ & $<8$ & $<20$ \\
\hline bis-2-Ethylhexyl adipate & $\mu \mathrm{g} / \mathrm{L}$ & $<8$ & $<4$ & $<4$ & $<4$ & $<8$ & $<8$ & $<8$ & $<8$ & $<8$ & $<20$ \\
\hline bis-2-Ethylhexyl phthalate (Dioctyl phthlate) & $\mu \mathrm{g} / \mathrm{L}$ & $<8$ & $<4$ & $<4$ & $<4$ & $<8$ & $<8$ & $<8$ & $<8$ & $<8$ & $<20$ \\
\hline 4-Ethylmorpholine & $\mu \mathrm{g} / \mathrm{L}$ & $<8$ & $<4$ & $<4$ & $<4$ & $<8$ & $<8$ & $<8$ & $<8$ & $<8$ & $<20$ \\
\hline 1-Formylpiperidine & $\mu \mathrm{g} / \mathrm{L}$ & $<8$ & $<4$ & $<4$ & $<4$ & $<8$ & $<8$ & $<8$ & $<8$ & $<8$ & $<20$ \\
\hline Heptanoic acid & $\mu \mathrm{g} / \mathrm{L}$ & $<8$ & $<4$ & $<4$ & $<4$ & $<8$ & $<24$ & $<24$ & $<8$ & $<8$ & $<20$ \\
\hline 2-Heptanone & $\mu \mathrm{g} / \mathrm{L}$ & $<8$ & $<4$ & $<4$ & $<4$ & $<8$ & $<8$ & $<8$ & $<8$ & $<8$ & $<20$ \\
\hline gamma-Hexalactone & $\mu \mathrm{g} / \mathrm{L}$ & $<8$ & $<4$ & $<4$ & $<4$ & $<8$ & $<8$ & $<8$ & $<8$ & $<8$ & $<20$ \\
\hline Hexanoic acid & $\mu \mathrm{g} / \mathrm{L}$ & $<16$ & $<8$ & $<8$ & $<8$ & $<16$ & $<24$ & $<24$ & $<16$ & $<16$ & $<40$ \\
\hline 2-Hexanol & $\mu \mathrm{g} / \mathrm{L}$ & $<8$ & $<4$ & $<4$ & $<4$ & $<8$ & $<8$ & $<8$ & $<8$ & $<8$ & $<20$ \\
\hline 2-Hydroxybenzothiazole & $\mu \mathrm{g} / \mathrm{L}$ & $<8$ & $<4$ & $<4$ & $<4$ & $<8$ & $<8$ & $<8$ & $<8$ & $<8$ & $<20$ \\
\hline Ibuprofen & $\mu \mathrm{g} / \mathrm{L}$ & $<8$ & $<4$ & $<4$ & $<4$ & $<8$ & $<24$ & $<24$ & $<8$ & $<8$ & $<20$ \\
\hline Iodoform & $\mu \mathrm{g} / \mathrm{L}$ & $<8$ & $<4$ & $<4$ & $<4$ & $<8$ & $<8$ & $<8$ & $<8$ & $<8$ & $<20$ \\
\hline Isophorone & $\mu \mathrm{g} / \mathrm{L}$ & $<8$ & $<4$ & $<4$ & $<4$ & $<8$ & $<8$ & $<8$ & $<8$ & $<8$ & $<20$ \\
\hline 4-Isopropylphenol & $\mu \mathrm{g} / \mathrm{L}$ & $<8$ & $<4$ & $<4$ & $<4$ & $<8$ & $<8$ & $<8$ & $<8$ & $<8$ & $<20$ \\
\hline Lauramide & $\mu \mathrm{g} / \mathrm{L}$ & $<8$ & $<4$ & $<4$ & $<4$ & $<8$ & $<8$ & $<8$ & $<8$ & $<8$ & $<20$ \\
\hline Lauric acid (Dodecanoic acid) & $\mu \mathrm{g} / \mathrm{L}$ & $<240$ & $<120$ & $<120$ & $<120$ & $<240$ & $<240$ & $<240$ & $<240$ & $<240$ & $<600$ \\
\hline p-Menth-1-en-8-ol (alpha-Terpineol) & $\mu \mathrm{g} / \mathrm{L}$ & $<8$ & $<4$ & $<4$ & $<4$ & $<8$ & $<8$ & $<8$ & $<8$ & $<8$ & $<20$ \\
\hline 2-Mercaptobenzothiazole & $\mu \mathrm{g} / \mathrm{L}$ & $<80$ & $<40$ & $\angle 40$ & $\angle 40$ & $<80$ & $<80$ & $<80$ & $<80$ & $<80$ & $<200$ \\
\hline 2-Methyl-2,4-pentanediol & $\mu \mathrm{g} / \mathrm{L}$ & $<8$ & $<4$ & $<4$ & $<4$ & $<8$ & $<8$ & $<8$ & $<8$ & $<8$ & $<20$ \\
\hline 1-Methyl-2-pyrrolidinone & $\mu \mathrm{g} / \mathrm{L}$ & $<8$ & $<4$ & $<4$ & $<4$ & $<8$ & $<8$ & $<8$ & $<8$ & $<8$ & $<20$ \\
\hline Methyl-4-hydroxybenzoate & $\mu \mathrm{g} / \mathrm{L}$ & $<8$ & $<4$ & $<4$ & $<4$ & $<8$ & $<8$ & $<8$ & $<8$ & $<8$ & $<20$ \\
\hline
\end{tabular}


Appendix 3. ISS WPA RIP and PWD Summary for Expeditions 21 through 25

\begin{tabular}{|c|c|c|c|c|c|c|c|c|c|c|c|}
\hline \multirow[t]{2}{*}{ Mission } & & \multicolumn{2}{|c|}{ ISS ULF4/Exp. 23} & \multicolumn{3}{|c|}{ Soyuz 22/Exp. 24} & \multicolumn{2}{|c|}{ Soyuz 23/Exp. 25} & \multicolumn{2}{|c|}{ ISS 19A/Exp. 23} & \multirow{2}{*}{\begin{tabular}{|c|} 
Soyuz 22/Exp. 24 \\
W PA RIP
\end{tabular}} \\
\hline & & $\begin{array}{c}\text { WPA PWD } \\
\text { Ambient }\end{array}$ & $\begin{array}{c}\text { WPA PWD } \\
\text { Ambient }\end{array}$ & $\begin{array}{c}\text { WPA PWD } \\
\text { Ambient }\end{array}$ & WPA PWD Hot & $\begin{array}{c}\text { WPA PWD } \\
\text { Ambient }\end{array}$ & WPA PWD Hot & & PWD Aux Port & WPA RIP & \\
\hline Sample Location & & & & & & & & & Processed & Proceserd & \\
\hline $\begin{array}{l}\text { Sample Description } \\
\text { Sample Date }\end{array}$ & & $\begin{array}{l}\text { Potable W ater } \\
4 / 26 / 2010\end{array}$ & $\begin{array}{c}\text { Potable Water } \\
5 / 18 / 2010\end{array}$ & $\begin{array}{c}\text { Potable W ater } \\
7 / 14 / 2010\end{array}$ & \begin{tabular}{|c|} 
Potable Water \\
$8 / 25 / 2010$
\end{tabular} & $\begin{array}{l}\text { Potable Water } \\
\text { 9/15/2010 }\end{array}$ & \begin{tabular}{|c} 
Potable Water \\
$10 / 19 / 2010$
\end{tabular} & $\begin{array}{c}\text { Potable Water } \\
11 / 23 / 2010\end{array}$ & $\begin{array}{c}\text { Water } \\
2 / 25 / 2010\end{array}$ & $\begin{array}{c}\text { Water } \\
2 / 25 / 2010\end{array}$ & $\begin{array}{l}\text { Processed Water } \\
\text { 7/29/2010 }\end{array}$ \\
\hline Analysis/Sample ID & Units & 20100527006 & 20100527007 & 20100926001 & 20100926002 & 20100926003 & 20101129001 & 20101129002 & 20100422009 & 20100422008 & 20100926004 \\
\hline Methyl sulfone & $\mu \mathrm{g} / \mathrm{L}$ & 40 & 34 & 36 & $<4$ & $<8$ & 48 & 50 & 88 & 98 & 150 \\
\hline 2-Methyl butyric acid & $\mu \mathrm{g} / \mathrm{L}$ & $<24$ & $<12$ & $<12$ & $<12$ & $<24$ & $<24$ & $<24$ & $<24$ & $<24$ & $<60$ \\
\hline 2-Methylthiobenzothiazole & $\mu \mathrm{g} / \mathrm{L}$ & $<8$ & $<4$ & $<4$ & $<4$ & $<8$ & $<8$ & $<8$ & $<8$ & $<8$ & $<20$ \\
\hline Monomethyl phthalate & $\mu \mathrm{g} / \mathrm{L}$ & $<8$ & $<4$ & $<4$ & $<4$ & $<8$ & $<8$ & $<8$ & $<8$ & $<8$ & $<20$ \\
\hline Myristic acid & $\mu \mathrm{g} / \mathrm{L}$ & $<48$ & $<24$ & $<24$ & $<24$ & $<48$ & $<64$ & $<64$ & $<48$ & $<48$ & $<120$ \\
\hline (t)-Neomenthol & $\mu \mathrm{g} / \mathrm{L}$ & $<8$ & $<4$ & $<4$ & $<4$ & $<8$ & $<8$ & $<8$ & $<8$ & $<8$ & $<20$ \\
\hline Nicotine & $\mu \mathrm{g} / \mathrm{L}$ & $<8$ & $<4$ & $<4$ & $<4$ & $<8$ & $<8$ & $<8$ & $<8$ & $<8$ & $<20$ \\
\hline \begin{tabular}{|l|} 
Nonadecane \\
\end{tabular} & $\mu \mathrm{g} / \mathrm{L}$ & $<8$ & $<4$ & $<4$ & $<4$ & $<8$ & $<8$ & $<8$ & $<8$ & $<8$ & $<20$ \\
\hline Nonanoic acid & $\mu \mathrm{g} / \mathrm{L}$ & $<24$ & $<12$ & $<12$ & $<12$ & $<24$ & $<24$ & $<24$ & $<24$ & $<24$ & $<60$ \\
\hline 1-Octadecanol & $\mu \mathrm{g} / \mathrm{L}$ & $<24$ & $<12$ & $<12$ & $<12$ & $<24$ & $<24$ & $<24$ & $<24$ & $<24$ & $<60$ \\
\hline Octamethylcyclotetrasiloxane & $\mu \mathrm{g} / \mathrm{L}$ & $<8$ & $<4$ & $<4$ & $<4$ & $<8$ & $<8$ & $<8$ & $<8$ & $<8$ & $<20$ \\
\hline Octanoic acid & $\mu \mathrm{g} / \mathrm{L}$ & $<16$ & $<8$ & $<8$ & $<8$ & $<16$ & $<48$ & $<48$ & $<16$ & $<16$ & $<40$ \\
\hline 4-tert-Octylphenol & $\mu \mathrm{g} / \mathrm{L}$ & $<8$ & $<4$ & $<4$ & $<4$ & $<8$ & $<8$ & $<8$ & $<8$ & $<8$ & $<20$ \\
\hline Oleic acid & $\mu \mathrm{g} / \mathrm{L}$ & $<80$ & $<40$ & $<40$ & $<40$ & $<80$ & $<80$ & $<80$ & $<80$ & $<80$ & $<200$ \\
\hline \begin{tabular}{|l|} 
Oxindole \\
\end{tabular} & $\mu \mathrm{g} / \mathrm{L}$ & $<8$ & $<4$ & $<4$ & $<4$ & $<8$ & $<8$ & $<8$ & $<8$ & $<8$ & $<20$ \\
\hline Palmitic acid & $\mu \mathrm{g} / \mathrm{L}$ & $<240$ & $<120$ & $<120$ & $<120$ & $<240$ & $<240$ & $<240$ & $<240$ & $<240$ & $<600$ \\
\hline Palmitoleic acid & $\mu \mathrm{g} / \mathrm{L}$ & NA & NA & NA & $\mathrm{NA}$ & NA & NA & $\mathrm{NA}$ & $\mathrm{NA}$ & NA & $\mathrm{NA}$ \\
\hline \begin{tabular}{|l|} 
Pentacosane \\
\end{tabular} & $\mu \mathrm{g} / \mathrm{L}$ & $<8$ & $<4$ & $<4$ & $<4$ & $<8$ & $<8$ & $<8$ & $<8$ & $<8$ & $<20$ \\
\hline \begin{tabular}{|l|} 
sec-Phenethyl alcohol \\
\end{tabular} & $\mu \mathrm{g} / \mathrm{L}$ & $<8$ & $<4$ & $<4$ & $<4$ & $<8$ & $<8$ & $<8$ & $<8$ & $<8$ & $<20$ \\
\hline Phenol & $\mu \mathrm{g} / \mathrm{L}$ & $<8$ & $<4$ & $<4$ & $<4$ & $<8$ & $<8$ & $<8$ & $<8$ & $<8$ & $<20$ \\
\hline 2-Phenoxyethanol & $\mu \mathrm{g} / \mathrm{L}$ & $<8$ & $<4$ & $<4$ & $<4$ & $<8$ & $<8$ & $<8$ & $<8$ & $<8$ & $<20$ \\
\hline N-Phenyl-2-naphthylamine & $\mu \mathrm{g} / \mathrm{L}$ & $<8$ & $<4$ & $<4$ & $<4$ & $<8$ & $<8$ & $<8$ & $<8$ & $<8$ & $<20$ \\
\hline 2-Phenyl-2-propanol & $\mu \mathrm{g} / \mathrm{L}$ & $<8$ & $<4$ & $<4$ & $<4$ & $<8$ & $<8$ & $<8$ & $<8$ & $<8$ & $<20$ \\
\hline 2-Phenylacetic acid & $\mu \mathrm{g} / \mathrm{L}$ & $<32$ & $<16$ & $<16$ & $<16$ & $<32$ & $<32$ & $<32$ & $<32$ & $<32$ & $<80$ \\
\hline \begin{tabular}{|l|} 
Phenethyl alcohol \\
\end{tabular} & $\mu \mathrm{g} / \mathrm{L}$ & $<8$ & $<4$ & $<4$ & $<4$ & $<8$ & $<8$ & $<8$ & $<8$ & $<8$ & $<20$ \\
\hline 2-Phenylphenol & $\mu \mathrm{g} / \mathrm{L}$ & $<8$ & $<4$ & $<4$ & $<4$ & $<8$ & $<8$ & $<8$ & $<8$ & $<8$ & $<20$ \\
\hline \begin{tabular}{|l|} 
Salicyclic Acid \\
\end{tabular} & $\mu \mathrm{g} / \mathrm{L}$ & $<64$ & $<32$ & $<32$ & $<32$ & $<64$ & $<64$ & $<64$ & $<64$ & $<64$ & $<160$ \\
\hline trans-Squalene & $\mu \mathrm{g} / \mathrm{L}$ & $\begin{array}{l}<16 \\
\end{array}$ & $<8$ & $<8$ & $<8$ & $\begin{array}{l}<16 \\
\end{array}$ & $\begin{array}{l}<16 \\
\end{array}$ & $<16$ & $\begin{array}{l}<16 \\
\end{array}$ & $<16$ & $<40$ \\
\hline Stearic acid & $\mu \mathrm{g} / \mathrm{L}$ & $\mathrm{NA}$ & $\mathrm{NA}$ & NA & $\mathrm{NA}$ & $\mathrm{NA}$ & NA & $\mathrm{NA}$ & $\mathrm{NA}$ & NA & $\mathrm{NA}$ \\
\hline 1-Tetradecanol & $\mu \mathrm{g} / \mathrm{L}$ & $<8$ & $<4$ & $<4$ & $<4$ & $<8$ & $<8$ & $<8$ & $<8$ & $<8$ & $<20$ \\
\hline Tetramethylsuccinonitrile & $\mu \mathrm{g} / \mathrm{L}$ & $<8$ & $<4$ & $<4$ & $<4$ & $<8$ & $<8$ & $<8$ & $<8$ & $<8$ & $<20$ \\
\hline \begin{tabular}{|l|} 
Tetramethyl thiourea \\
\end{tabular} & $\mu \mathrm{g} / \mathrm{L}$ & $<8$ & $<4$ & $<4$ & $<4$ & $<8$ & $<8$ & $<8$ & $<8$ & $<8$ & $<20$ \\
\hline Tetramethylurea & $\mu \mathrm{g} / \mathrm{L}$ & $<8$ & $<4$ & $<4$ & $<4$ & $<8$ & $<8$ & $<8$ & $<8$ & $<8$ & $<20$ \\
\hline Thymol & $\mu \mathrm{g} / \mathrm{L}$ & $<8$ & $<4$ & $<4$ & $<4$ & $<8$ & $<8$ & $<8$ & $<8$ & $<8$ & $<20$ \\
\hline $1,3,5-$-Triallyl-1,3,5-triazine-2,4,6(1H,3H,5H)-trione & $\mu \mathrm{g} / \mathrm{L}$ & $<8$ & $<4$ & $<4$ & $<4$ & $<8$ & $<8$ & $<8$ & $<8$ & $<8$ & $<20$ \\
\hline Tributylamine & $\mu \mathrm{g} / \mathrm{L}$ & $<8$ & $<4$ & $<4$ & $<4$ & $<8$ & $<8$ & $<8$ & $<8$ & $<8$ & $<20$ \\
\hline Tributyl phosphate & $\mu \mathrm{g} / \mathrm{L}$ & $<8$ & $<4$ & $<4$ & $<4$ & $<8$ & $<8$ & $<8$ & $<8$ & $<8$ & $<20$ \\
\hline Triethyl phosphate & $\mu \mathrm{g} / \mathrm{L}$ & $<16$ & $<8$ & $<8$ & $<8$ & $<16$ & $<16$ & $<16$ & $<16$ & $<16$ & $<40$ \\
\hline 2,2,4-Trimethyl-1,3-pentanediol diisobutyrate & $\mu \mathrm{g} / \mathrm{L}$ & $<16$ & $<8$ & $<8$ & $<8$ & $<16$ & $<16$ & $<16$ & $<16$ & $<16$ & $<40$ \\
\hline Tripropylene glycol monomethyl ether & $\mu \mathrm{g} / \mathrm{L}$ & $<8$ & $<4$ & $<4$ & $<4$ & $<8$ & $<8$ & $<8$ & $<8$ & $<8$ & $<20$ \\
\hline Undecanoic acid & $\mu \mathrm{g} / \mathrm{L}$ & $<48$ & $<24$ & $<24$ & $<24$ & $<48$ & $<48$ & $<48$ & $<48$ & $<48$ & $<120$ \\
\hline 2-Undecanone & $\mu \mathrm{g} / \mathrm{L}$ & $<8$ & $<4$ & $<4$ & $<4$ & $<8$ & $<8$ & $<8$ & $<8$ & $<8$ & $<20$ \\
\hline Valeric acid (Pentanoic acid) & $\mu \mathrm{g} / \mathrm{L}$ & $<48$ & $<24$ & $<24$ & $<24$ & $<48$ & $<48$ & $<48$ & $<48$ & $<48$ & $<120$ \\
\hline Vanillin & $\mu \mathrm{g} / \mathrm{L}$ & $<16$ & $<8$ & $<8$ & $<8$ & $<16$ & $<16$ & $<16$ & $<16$ & $<16$ & $<40$ \\
\hline & & & & & & & & & & & \\
\hline
\end{tabular}


Appendix 3. ISS WPA RIP and PWD Summary for Expeditions 21 through 25

\begin{tabular}{|c|c|c|c|c|c|c|c|c|c|c|c|}
\hline \multirow[t]{2}{*}{ Mission } & \multirow[b]{3}{*}{ Units } & \multicolumn{2}{|c|}{ ISS ULF4/Exp. 23} & \multicolumn{3}{|c|}{ Soyuz 22/Exp. 24} & \multicolumn{2}{|c|}{ Soyuz 23/Exp. 25} & \multicolumn{2}{|c|}{ ISS 19A/Exp. 23} & \multirow{3}{*}{\begin{tabular}{|c|} 
Soyuz 22/Exp. 24 \\
W PA RIP \\
\\
\\
Processed Water \\
$7 / 29 / 2010$ \\
20100926004 \\
\end{tabular}} \\
\hline & & $\begin{array}{c}\text { WPA PWD } \\
\text { Ambient }\end{array}$ & $\begin{array}{c}\text { WPA PWD } \\
\text { Ambient }\end{array}$ & $\begin{array}{c}\text { WPA PWD } \\
\text { Ambient }\end{array}$ & W PA PWD Hot & $\begin{array}{c}\text { WPA PWD } \\
\text { Ambient }\end{array}$ & W PA PWD Hot & W PA PW D Hot & PWD Aux Port & W PA RIP & \\
\hline $\begin{array}{l}\text { Sample Location } \\
\\
\text { Sample Description } \\
\text { Sample Date } \\
\text { Analysis/Sample ID }\end{array}$ & & $\begin{array}{c}\text { Potable W ater } \\
4 / 26 / 2010 \\
20100527006\end{array}$ & $\begin{array}{c}\text { Potable W ater } \\
5 / 18 / 2010 \\
20100527007\end{array}$ & $\begin{array}{l}\text { Potable W ater } \\
7 / 14 / 2010 \\
20100926001\end{array}$ & \begin{tabular}{|c|} 
Potable W ater \\
$8 / 25 / 2010$ \\
20100926002 \\
\end{tabular} & $\begin{array}{l}\text { Potable Water } \\
9 / 15 / 2010 \\
20100926003\end{array}$ & \begin{tabular}{|c|} 
Potable W ater \\
$10 / 19 / 2010$ \\
20101129001 \\
\end{tabular} & \begin{tabular}{|c|} 
Potable Water \\
$11 / 23 / 2010$ \\
20101129002
\end{tabular} & $\begin{array}{c}\text { Processed } \\
\text { Water } \\
2 / 25 / 2010 \\
20100422009\end{array}$ & $\begin{array}{c}\text { Processed } \\
\text { Water } \\
2 / 25 / 2010 \\
20100422008\end{array}$ & \\
\hline \multicolumn{12}{|c|}{ Acid Extractables-EPA 625 List } \\
\hline 4-Chloro-3-methylphenol & $\mu \mathrm{g} / \mathrm{L}$ & $<16$ & $<8$ & $<8$ & $<8$ & $<16$ & $<16$ & $<16$ & $<16$ & $<16$ & $\angle 40$ \\
\hline 2-Chlorophenol & $\mu \mathrm{g} / \mathrm{L}$ & $<16$ & $<8$ & $<8$ & $<8$ & $<16$ & $<16$ & $<16$ & $<16$ & $<16$ & $\angle 40$ \\
\hline 2,4-Dichlorophenol & $\mu \mathrm{g} / \mathrm{L}$ & $<16$ & $<8$ & $<8$ & $<8$ & $<16$ & $<16$ & $<16$ & $<16$ & $<16$ & $<40$ \\
\hline 2,4-Dimethylphenol & $\mu \mathrm{g} / \mathrm{L}$ & $<16$ & $<8$ & $<8$ & $<8$ & $<16$ & $<16$ & $<16$ & $<16$ & $<16$ & $\angle 40$ \\
\hline 2,4-Dinitrophenol & $\mu \mathrm{g} / \mathrm{L}$ & $<16$ & $<8$ & $<8$ & $<8$ & $<16$ & $<16$ & $<16$ & $<16$ & $<16$ & $\angle 40$ \\
\hline 2-Methyl-4,6-dinitrophenol & $\mu \mathrm{g} / \mathrm{L}$ & $<16$ & $<8$ & $<8$ & $<8$ & $<16$ & $<16$ & $<16$ & $<16$ & $<16$ & $<40$ \\
\hline 2-Nitrophenol & $\mu \mathrm{g} / \mathrm{L}$ & $<16$ & $<8$ & $<8$ & $<8$ & $<16$ & $<16$ & $<16$ & $<16$ & $<16$ & $<40$ \\
\hline 4-Nitrophenol & $\mu \mathrm{g} / \mathrm{L}$ & $<16$ & $<8$ & $<8$ & $<8$ & $<16$ & $<16$ & $<16$ & $<16$ & $<16$ & $\angle 40$ \\
\hline Pentachlorophenol & $\mu \mathrm{g} / \mathrm{L}$ & $<16$ & $<8$ & $<8$ & $<8$ & $<16$ & $<16$ & $<16$ & $<16$ & $<16$ & $\angle 40$ \\
\hline Phenol & $\mu \mathrm{g} / \mathrm{L}$ & $<8$ & $<4$ & $<4$ & $<4$ & $<8$ & $<8$ & $<8$ & $<8$ & $<8$ & $<20$ \\
\hline 2,4,5-Trichlorophenol & $\mu \mathrm{g} / \mathrm{L}$ & $<16$ & $<8$ & $<8$ & $<8$ & $<16$ & $<16$ & $<16$ & $<16$ & $<16$ & $\angle 40$ \\
\hline 2,4,6-Trichlorophenol & $\mu \mathrm{g} / \mathrm{L}$ & $<16$ & $<8$ & $<8$ & $<8$ & $<16$ & $<16$ & $<16$ & $<16$ & $<16$ & $\angle 40$ \\
\hline 4-Methylphenol & $\mu \mathrm{g} / \mathrm{L}$ & $<8$ & $<4$ & $<4$ & $<4$ & $<8$ & $<8$ & $<8$ & $<8$ & $<8$ & $<20$ \\
\hline & & & & & & & & & & & \\
\hline \multicolumn{12}{|c|}{ Base/Neutral Extractables - EPA 625 List } \\
\hline Benzidine & $\mu \mathrm{g} / \mathrm{L}$ & $<16$ & $<8$ & $<8$ & $<8$ & $<16$ & $<16$ & $<16$ & $<16$ & $<16$ & $\angle 40$ \\
\hline 3,3-Dichlorobenzidine & $\mu \mathrm{g} / \mathrm{L}$ & $<16$ & $<8$ & $<8$ & $<8$ & $<16$ & $<16$ & $<16$ & $<16$ & $<16$ & $\angle 40$ \\
\hline bis-(2-Ethylhexyl)phthalate & $\mu \mathrm{g} / \mathrm{L}$ & $<8$ & $<4$ & $<4.0$ & $<4.0$ & $<8$ & $<8$ & $<8$ & $<8$ & $<8$ & $<20$ \\
\hline Benzyl butyl phthalate & $\mu \mathrm{g} / \mathrm{L}$ & $<8$ & $<4$ & $<4$ & $<4$ & $<8$ & $<8$ & $<8$ & $<8$ & $<8$ & $<20$ \\
\hline Dibutylphthalate & $\mu \mathrm{g} / \mathrm{L}$ & $<8$ & $<4$ & $<4$ & $<4$ & $<8$ & $<8$ & $<8$ & $<8$ & $<8$ & $<20$ \\
\hline Diethylphthalate & $\mu \mathrm{g} / \mathrm{L}$ & $<8$ & $<4$ & $<4$ & $<4$ & $<8$ & $<8$ & $<8$ & $<8$ & $<8$ & $<20$ \\
\hline Dimethylphthalate & $\mu \mathrm{g} / \mathrm{L}$ & $<8$ & $<4$ & $<4$ & $<4$ & $<8$ & $<8$ & $<8$ & $<8$ & $<8$ & $<20$ \\
\hline \begin{tabular}{|l} 
Di-n-octyl phthalate \\
\end{tabular} & $\mu \mathrm{g} / \mathrm{L}$ & $<16$ & $<8$ & $<8$ & $<8$ & $<16$ & $<16$ & $<16$ & $<16$ & $<16$ & $\angle 40$ \\
\hline N-Nitrosodimethylamine & $\mu \mathrm{g} / \mathrm{L}$ & $<16$ & $<8$ & $<8$ & $<8$ & $<16$ & $<16$ & $<16$ & $<16$ & $<16$ & $<40$ \\
\hline \begin{tabular}{|l|} 
N-Nitrosodiphenylamine \\
\end{tabular} & $\mu \mathrm{g} / \mathrm{L}$ & $<16$ & $<8$ & $<8$ & $<8$ & $<16$ & $<16$ & $<16$ & $<16$ & $<16$ & $<40$ \\
\hline N-Nitrosodi-n-propylamine & $\mu \mathrm{g} / \mathrm{L}$ & $<16$ & $<8$ & $<8$ & $<8$ & $<16$ & $<16$ & $<16$ & $<16$ & $<16$ & $\angle 40$ \\
\hline 2,4-Dinitrotoluene & $\mu \mathrm{g} / \mathrm{L}$ & $<16$ & $<8$ & $<8$ & $<8$ & $<16$ & $<16$ & $<16$ & $<16$ & $<16$ & $\angle 40$ \\
\hline 2,6-Dinitrotoluene & $\mu \mathrm{g} / \mathrm{L}$ & $<16$ & $<8$ & $<8$ & $<8$ & $<16$ & $<16$ & $<16$ & $<16$ & $<16$ & $\angle 40$ \\
\hline Isophorone & $\mu \mathrm{g} / \mathrm{L}$ & $<8$ & $<4$ & $<4$ & $<4$ & $<8$ & $<8$ & $<8$ & $<8$ & $<8$ & $<20$ \\
\hline \begin{tabular}{|l|} 
Nitrobenzene \\
\end{tabular} & $\mu \mathrm{g} / \mathrm{L}$ & $<16$ & $<8$ & $<8$ & $<8$ & $<16$ & $<16$ & $<16$ & $<16$ & $<16$ & $<40$ \\
\hline Acenaphthene & $\mu \mathrm{g} / \mathrm{L}$ & $<16$ & $<8$ & $\mathrm{NA}$ & $\mathrm{NA}$ & $\mathrm{NA}$ & $<16$ & $<16$ & $<16$ & $<16$ & $\mathrm{NA}$ \\
\hline Acenaphthylene & $\mu \mathrm{g} / \mathrm{L}$ & $<16$ & $<8$ & $<8$ & $<8$ & $<16$ & $<16$ & $<16$ & $<16$ & $<16$ & $<40$ \\
\hline Anthracene & $\mu \mathrm{g} / \mathrm{L}$ & $<16$ & $<8$ & $<8$ & $<8$ & $<16$ & $<16$ & $<16$ & $<16$ & $<16$ & $<40$ \\
\hline Benzo(a)anthracene & $\mu \mathrm{g} / \mathrm{L}$ & $<16$ & $<8$ & $<8$ & $<8$ & $<16$ & $<16$ & $<16$ & $<16$ & $<16$ & $<40$ \\
\hline Benzo(a)pyrene & $\mu \mathrm{g} / \mathrm{L}$ & $<10$ & $<5$ & $<5$ & $<5$ & $<10$ & $<10$ & $<10$ & $<10$ & $<10$ & $<25$ \\
\hline Benzo(b)fluoranthene & $\mu \mathrm{g} / \mathrm{L}$ & $<8$ & $<4$ & $<4$ & $<4$ & $<8$ & $<8$ & $<8$ & $<8$ & $<8$ & $<20$ \\
\hline Benzo(ghi)perylene & $\mu \mathrm{g} / \mathrm{L}$ & $<10$ & $<5$ & $<5$ & $<5$ & $<10$ & $<10$ & $<10$ & $<10$ & $<10$ & $<25$ \\
\hline Benzo(k)fluoroanthene & $\mu \mathrm{g} / \mathrm{L}$ & $<8$ & $<4$ & $<4$ & $<4$ & $<8$ & $<8$ & $<8$ & $<8$ & $<8$ & $<20$ \\
\hline Chrysene & $\mu \mathrm{g} / \mathrm{L}$ & $<20$ & $<10$ & $<10$ & $<10$ & $<20$ & $<20$ & $<20$ & $<20$ & $<20$ & $<50$ \\
\hline Dibenzo(a,h)anthracene & $\mu \mathrm{g} / \mathrm{L}$ & $<10$ & $<5$ & $<5$ & $<5$ & $<10$ & $<10$ & $<10$ & $<1$ & $<10$ & $<25$ \\
\hline Fluoranthene & $\mu \mathrm{g} / \mathrm{L}$ & $<8$ & $<4$ & $<4$ & $<4$ & $<8$ & $<8$ & $<8$ & $<8$ & $<8$ & $<20$ \\
\hline Fluorene & $\mu \mathrm{g} / \mathrm{L}$ & $<16$ & $<8$ & $<8$ & $<8$ & $<16$ & $<16$ & $<16$ & $<16$ & $<16$ & $\angle 40$ \\
\hline Indeno(1,2,3-cd)pyrene & $\mu \mathrm{g} / \mathrm{L}$ & $<10$ & $<5$ & $<5$ & $<5$ & $<10$ & $<10$ & $<10$ & $<10$ & $<10$ & $<25$ \\
\hline Naphthalene & $\mu \mathrm{g} / \mathrm{L}$ & $<40$ & $<20$ & $<20$ & $<20$ & $\angle 40$ & $\angle 40$ & $<40$ & $\angle 40$ & $<40$ & $<100$ \\
\hline Phenanthrene & $\mu \mathrm{g} / \mathrm{L}$ & $<8$ & $<4$ & $<4$ & $<4$ & $<8$ & $<8$ & $<8$ & $<8$ & $<8$ & $<20$ \\
\hline Pyrene & $\mu \mathrm{g} / \mathrm{L}$ & $<8$ & $<4$ & $<4$ & $<4$ & $<8$ & $<8$ & $<8$ & $<8$ & $<8$ & $<20$ \\
\hline bis(2-Chloroethyl) ether & $\mu \mathrm{g} / \mathrm{L}$ & $<16$ & $<8$ & $<8$ & $<8$ & $<16$ & $<16$ & $<16$ & $<16$ & $<16$ & $\angle 40$ \\
\hline
\end{tabular}


Appendix 3. ISS WPA RIP and PWD Summary for Expeditions 21 through 25

\begin{tabular}{|c|c|c|c|c|c|c|c|c|c|c|c|}
\hline \multirow[t]{2}{*}{ Mission } & \multirow[b]{3}{*}{ Units } & \multicolumn{2}{|c|}{ ISS ULF4/Exp. 23} & \multicolumn{3}{|c|}{ Soyuz 22/Exp. 24 } & \multicolumn{2}{|c|}{ Soyuz 23/Exp. 25} & \multicolumn{2}{|c|}{ ISS 19A/Exp. 23} & \multirow{2}{*}{\begin{tabular}{|c|} 
Soyuz 22/Exp. 24 \\
W PA RIP
\end{tabular}} \\
\hline & & $\begin{array}{l}\text { WPA PWD } \\
\text { Ambient }\end{array}$ & $\begin{array}{l}\text { WPA PWD } \\
\text { Ambient }\end{array}$ & $\begin{array}{c}\text { WPA PWD } \\
\text { Ambient }\end{array}$ & W PA PWD Hot & $\begin{array}{c}\text { WPA PWD } \\
\text { Ambient }\end{array}$ & W PA PWD Hot & W PA PW D Hot & PWD Aux Port & WPA RIP & \\
\hline $\begin{array}{l}\text { Sample Location } \\
\\
\text { Sample Description } \\
\text { Sample Date } \\
\text { Analysis/Sample ID }\end{array}$ & & $\begin{array}{c}\text { Potable Water } \\
4 / 26 / 2010 \\
20100527006\end{array}$ & $\begin{array}{c}\text { Potable W ater } \\
5 / 18 / 2010 \\
20100527007\end{array}$ & $\begin{array}{l}\text { Potable W ater } \\
7 / 14 / 2010 \\
20100926001\end{array}$ & \begin{tabular}{|c|} 
Potable $W$ ater \\
$8 / 25 / 2010$ \\
20100926002 \\
\end{tabular} & $\begin{array}{c}\text { Potable W ater } \\
9 / 15 / 2010 \\
20100926003\end{array}$ & \begin{tabular}{|c|} 
Potable W ater \\
$10 / 19 / 2010$ \\
20101129001 \\
\end{tabular} & \begin{tabular}{|c|} 
Potable Water \\
$11 / 23 / 2010$ \\
20101129002
\end{tabular} & \begin{tabular}{c|} 
Processed \\
Water \\
$2 / 25 / 2010$ \\
20100422009
\end{tabular} & $\begin{array}{c}\text { Processed } \\
\text { Water } \\
2 / 25 / 2010 \\
20100422008 \\
\end{array}$ & $\begin{array}{c}\text { Processed Water } \\
7 / 29 / 2010 \\
20100926004\end{array}$ \\
\hline bis(2-Chloroethoxy) methane & $\mu \mathrm{g} / \mathrm{L}$ & $<16$ & $<8$ & $<8$ & $<8$ & $<16$ & \begin{tabular}{|l|}
$<16$ \\
\end{tabular} & $<16$ & $<16$ & $<16$ & $<40$ \\
\hline bis(2-Chloroisopropyl) ether & $\mu \mathrm{g} / \mathrm{L}$ & $<16$ & $<8$ & $<8$ & $<8$ & $<16$ & $<16$ & $<16$ & $<16$ & $<16$ & $\angle 40$ \\
\hline 4-Bromophenyl phenyl ether & $\mu \mathrm{g} / \mathrm{L}$ & $<16$ & $<8$ & $<8$ & $<8$ & $<16$ & $<16$ & $<16$ & $<16$ & $<16$ & $<40$ \\
\hline 4-Chlorophenyl phenyl ether & $\mu \mathrm{g} / \mathrm{L}$ & $<16$ & $<8$ & $<8$ & $<8$ & $<16$ & $<16$ & $<16$ & $<16$ & $<16$ & $<40$ \\
\hline 2-Chloronaphthalene & $\mu \mathrm{g} / \mathrm{L}$ & $<16$ & $<8$ & $<8$ & $<8$ & $<16$ & $<16$ & $<16$ & $<16$ & $<16$ & $\angle 40$ \\
\hline 1,2-Dichlorobenzene & $\mu \mathrm{g} / \mathrm{L}$ & $<16$ & $<8$ & $<8$ & $<8$ & $<16$ & $<16$ & $<16$ & $<16$ & $<16$ & $\angle 40$ \\
\hline 1,3-Dichlorobenzene & $\mu \mathrm{g} / \mathrm{L}$ & $<16$ & $<8$ & $<8$ & $<8$ & $<16$ & $<16$ & $<16$ & $<16$ & $<16$ & $\angle 40$ \\
\hline 1,4-Dichlorobenzene & $\mu \mathrm{g} / \mathrm{L}$ & $<16$ & $<8$ & $<8$ & $<8$ & $<16$ & $<16$ & $<16$ & $<16$ & $<16$ & $<40$ \\
\hline Hexachlorobenzene & $\mu \mathrm{g} / \mathrm{L}$ & $<16$ & $<8$ & $<8$ & $<8$ & $<16$ & $<16$ & $<16$ & $<16$ & $<16$ & $\angle 40$ \\
\hline Hexachlorobutadiene & $\mu \mathrm{g} / \mathrm{L}$ & $<16$ & $<8$ & $<8$ & $<8$ & $<16$ & $<16$ & $<16$ & $<16$ & $<16$ & $\angle 40$ \\
\hline Hexachlorocyclopentadiene & $\mu \mathrm{g} / \mathrm{L}$ & $<16$ & $<8$ & $<8$ & $<8$ & $<16$ & $<16$ & $<16$ & $<16$ & $<16$ & $\angle 40$ \\
\hline Hexachloroethane & $\mu \mathrm{g} / \mathrm{L}$ & $<16$ & $<8$ & $<8$ & $<8$ & $<16$ & $<16$ & $<16$ & $<16$ & $<16$ & $<40$ \\
\hline 1,2,4-Trichlorobenzene & $\mu \mathrm{g} / \mathrm{L}$ & $<16$ & $<8$ & $<8$ & $<8$ & $<16$ & $<16$ & $<16$ & $<16$ & $<16$ & $\angle 40$ \\
\hline \multicolumn{12}{|l|}{ Alcohols (DAI/GC/MS) } \\
\hline 1-Butanol & $\mu \mathrm{g} / \mathrm{L}$ & $\angle 100$ & $\angle 100$ & $<100$ & $<100$ & $<100$ & $<100$ & $<100$ & $<100$ & $<100$ & $<100$ \\
\hline 2-Butanol & $\mu \mathrm{g} / \mathrm{L}$ & $<100$ & $<100$ & $<100$ & $<100$ & $<100$ & $<100$ & $<100$ & $<100$ & $<100$ & $<100$ \\
\hline Ethanol & $\mu \mathrm{g} / \mathrm{L}$ & $<100$ & $<100$ & $<100$ & $<100$ & $<100$ & $<100$ & $<100$ & $<100$ & $<100$ & $<100$ \\
\hline Methanol & $\mu \mathrm{g} / \mathrm{L}$ & $<100$ & $<100$ & $<100$ & $<100$ & $<100$ & $<100$ & $<100$ & $<100$ & $<100$ & $<100$ \\
\hline 2-Methyl-1-butanol & $\mu \mathrm{g} / \mathrm{L}$ & $<100$ & $<100$ & $<100$ & $<100$ & $<100$ & $<100$ & $<100$ & $<100$ & $<100$ & $<100$ \\
\hline 2-Methyl-2-butanol & $\mu \mathrm{g} / \mathrm{L}$ & $<100$ & $<100$ & $<100$ & $<100$ & $<100$ & $<100$ & $<100$ & $<100$ & $<100$ & $<100$ \\
\hline 3-Methyl-1-butanol (Isopentanol) & $\mu \mathrm{g} / \mathrm{L}$ & $<100$ & $<100$ & $<100$ & $<100$ & $<100$ & $<100$ & $<100$ & $<100$ & $<100$ & $<100$ \\
\hline 2-Methyl-1-propanol & $\mu \mathrm{g} / \mathrm{L}$ & $<100$ & $<100$ & $<100$ & $<100$ & $<100$ & $<100$ & $<100$ & $<100$ & $<100$ & $<100$ \\
\hline 2-Methyl-2-propanol & $\mu \mathrm{g} / \mathrm{L}$ & $<100$ & $<100$ & $<100$ & $<100$ & $<100$ & $<100$ & $<100$ & $<100$ & $<100$ & $<100$ \\
\hline 1-Pentanol (Amyl alcohol) & $\mu \mathrm{g} / \mathrm{L}$ & $<100$ & $<100$ & $<100$ & $<100$ & $<100$ & $<100$ & $<100$ & $<100$ & $<100$ & $<100$ \\
\hline 2-Pentanol (sec-Amyl alcohol) & $\mu \mathrm{g} / \mathrm{L}$ & $<100$ & $<100$ & $<100$ & $<100$ & $<100$ & $<100$ & $<100$ & $<100$ & $<100$ & $<100$ \\
\hline 3-Pentanol & $\mu \mathrm{g} / \mathrm{L}$ & $<100$ & $<100$ & $<100$ & $<100$ & $<100$ & $<100$ & $<100$ & $<100$ & $<100$ & $<100$ \\
\hline 1-Propanol & $\mu \mathrm{g} / \mathrm{L}$ & $<100$ & $<100$ & $\angle 100$ & $<100$ & $<100$ & $<100$ & $<100$ & $<100$ & $<100$ & $<100$ \\
\hline 2-Propanol (Isopropanol) & $\mu \mathrm{g} / \mathrm{L}$ & $<100$ & $<100$ & $<100$ & $<100$ & $<100$ & $<100$ & $<100$ & $<100$ & $<100$ & $<100$ \\
\hline & & & & & & & & & & & \\
\hline \multicolumn{12}{|l|}{ Glycols (DAI/GC/MS) } \\
\hline 1,2-Ethanediol (Ethylene glycol) & $\mu \mathrm{g} / \mathrm{L}$ & $<1000$ & $<1000$ & $<1000$ & $<1000$ & $<1000$ & $<1000$ & $<1000$ & $<1000$ & $<1000$ & $<1000$ \\
\hline 1,2-Propanediol (Propylene glycol) & $\mu \mathrm{g} / \mathrm{L}$ & $<500$ & $<500$ & $<500$ & $<500$ & $\angle 500$ & $\angle 500$ & $<500$ & $\angle 500$ & $\angle 500$ & $\angle 500$ \\
\hline \multicolumn{12}{|l|}{ Glycerol (LC/MS/MS) } \\
\hline Glycerol (1,2,3-Propanetriol) & $\mu \mathrm{g} / \mathrm{L}$ & $\mathrm{NA}$ & $\mathrm{NA}$ & $<300$ & $<300$ & $<300$ & $\angle 300$ & $\angle 300$ & $\mathrm{NA}$ & $\mathrm{NA}$ & $\angle 300$ \\
\hline & & & & & & & & & & & \\
\hline \multicolumn{12}{|c|}{ Silanols (GC/MS \& LC/MS/MS) (R\&D Method - NIST tracea } \\
\hline Dimethylsilanediol (DMSD) & $\mu \mathrm{g} / \mathrm{L}$ & $\mathrm{NA}$ & $\mathrm{NA}$ & 6100 & 8240 & 4730 & 8490 & $<400$ & $\mathrm{NA}$ & $\mathrm{NA}$ & 7340 \\
\hline & & & & & & & & & & & \\
\hline \multicolumn{12}{|l|}{ Carboxylates (CE) } \\
\hline Acetate & $\mu \mathrm{g} / \mathrm{L}$ & $<125$ & $<125$ & $<125$ & $<125$ & $<125$ & $<125$ & $<125$ & $<125$ & $<125$ & $<125$ \\
\hline Formate & $\mu \mathrm{g} / \mathrm{L}$ & $<125$ & $<125$ & $<125$ & $<125$ & $<125$ & $<125$ & $<125$ & $<125$ & $<125$ & $<125$ \\
\hline Glycolate & $\mu \mathrm{g} / \mathrm{L}$ & $<125$ & $<125$ & $<125$ & $<125$ & $<125$ & $<125$ & $<125$ & $<125$ & $<125$ & $<125$ \\
\hline \begin{tabular}{|l|} 
Glyoxylate \\
\end{tabular} & $\mu \mathrm{g} / \mathrm{L}$ & $<125$ & $<125$ & $<125$ & $<125$ & $<125$ & $<125$ & $<125$ & $<125$ & $<125$ & $<125$ \\
\hline Lactate & $\mu \mathrm{g} / \mathrm{L}$ & $<1000$ & $<1000$ & $<1000$ & $<1000$ & $<1000$ & $<1000$ & $<1000$ & $<1000$ & $<1000$ & $<1000$ \\
\hline Oxalate & $\mu \mathrm{g} / \mathrm{L}$ & $<125$ & $<125$ & $<125$ & $<125$ & $<125$ & $<125$ & $<125$ & $<125$ & $<125$ & $<125$ \\
\hline Propionate & $\mu \mathrm{g} / \mathrm{L}$ & $<125$ & $<125$ & $<125$ & $<125$ & $<125$ & $<125$ & $<125$ & $<125$ & $<125$ & $<125$ \\
\hline
\end{tabular}


Appendix 3. ISS WPA RIP and PWD Summary for Expeditions 21 through 25

\begin{tabular}{|c|c|c|c|c|c|c|c|c|c|c|c|}
\hline \multirow[t]{2}{*}{ Mission } & \multirow[b]{3}{*}{ Units } & \multicolumn{2}{|c|}{ ISS ULF4/Exp. 23} & \multicolumn{3}{|c|}{ Soyuz 22/Exp. 24} & \multicolumn{2}{|c|}{ Soyuz 23/Exp. 25} & \multicolumn{2}{|c|}{ ISS 19A/Exp. 23} & \multirow{3}{*}{$\begin{array}{c}\text { Soyuz 22/Exp. } 24 \\
\text { W PA RIP } \\
\\
\text { Processed Water } \\
7 / 29 / 2010 \\
20100926004 \\
\end{array}$} \\
\hline & & $\begin{array}{c}\text { WPA PWD } \\
\text { Ambient }\end{array}$ & $\begin{array}{c}\text { WPA PWD } \\
\text { Ambient }\end{array}$ & $\begin{array}{c}\text { W PA PWD } \\
\text { Ambient }\end{array}$ & WPA PWD Hot & $\begin{array}{c}\text { WPA PWD } \\
\text { Ambient }\end{array}$ & WPA PWD Hot & WPA PWD Hot & PWD Aux Port & W PA RIP & \\
\hline $\begin{array}{l}\text { Sample Location } \\
\text { Sample Description } \\
\text { Sample Date } \\
\text { Analysis/Sample ID }\end{array}$ & & $\begin{array}{c}\text { Potable W ater } \\
4 / 26 / 2010 \\
20100527006\end{array}$ & $\begin{array}{c}\text { Potable W ater } \\
5 / 18 / 2010 \\
20100527007\end{array}$ & $\begin{array}{c}\text { Potable W ater } \\
7 / 14 / 2010 \\
20100926001\end{array}$ & \begin{tabular}{|c|} 
Potable W ater \\
$8 / 25 / 2010$ \\
20100926002 \\
\end{tabular} & $\begin{array}{c}\text { Potable W ater } \\
9 / 15 / 2010 \\
20100926003\end{array}$ & $\begin{array}{c}\text { Potable W ater } \\
10 / 19 / 2010 \\
20101129001 \\
\end{array}$ & \begin{tabular}{|c|} 
Potable Water \\
$11 / 23 / 2010$ \\
20101129002 \\
\end{tabular} & $\begin{array}{c}\text { Processed } \\
\text { Water } \\
2 / 25 / 2010 \\
20100422009 \\
\end{array}$ & $\begin{array}{c}\text { Processed } \\
\text { Water } \\
2 / 25 / 2010 \\
20100422008 \\
\end{array}$ & \\
\hline & & & & & & & & & & & \\
\hline \multicolumn{12}{|l|}{\begin{tabular}{|l|} 
Aldehydes \\
\end{tabular}} \\
\hline Formaldehyde & $\mu \mathrm{g} / \mathrm{L}$ & 6 & 3 & 8 & 5 & 6 & $<5$ & $<5$ & 3 & 6 & 8 \\
\hline \multicolumn{12}{|l|}{ Amines (CE) } \\
\hline Ethylamine & $\mu \mathrm{g} / \mathrm{L}$ & $<125$ & $<125$ & $<125$ & $<125$ & $<125$ & $<125$ & $<125$ & $<125$ & $<125$ & $<125$ \\
\hline Methylamine & $\mu \mathrm{g} / \mathrm{L}$ & $<125$ & $<125$ & $<125$ & $<125$ & $<125$ & $<125$ & $<125$ & $<125$ & $<125$ & $<125$ \\
\hline n-Propylamine & $\mu \mathrm{g} / \mathrm{L}$ & $<125$ & $<125$ & $<125$ & $<125$ & $<125$ & $<125$ & $<125$ & $<125$ & $<125$ & $<125$ \\
\hline Trimethylamine & $\mu \mathrm{g} / \mathrm{L}$ & $<125$ & $<125$ & $<125$ & $<125$ & $<125$ & $<125$ & $\leq 125$ & $<125$ & $<125$ & $<125$ \\
\hline \multicolumn{11}{|l|}{ Non-volatiles (LC/UV-VIS) } & \\
\hline Urea & $\mu \mathrm{g} / \mathrm{L}$ & $<800$ & $<800$ & $<800$ & $<800$ & $<800$ & $<800$ & $<800$ & $<800$ & $<800$ & $<800$ \\
\hline Caprolactam & $\mu \mathrm{g} / \mathrm{L}$ & $<8$ & $<4$ & $<4$ & $<4$ & $<8$ & $<16$ & $<16$ & $<8$ & $<8$ & $<20$ \\
\hline & & & & & & & & & & & \\
\hline Organic Carbon Recovery & percent & 12.07 & 4.43 & 106.01 & 98.19 & 111.42 & 90.19 & 8.68 & 16.63 & 16.44 & 89.58 \\
\hline Unaccounted Organic Carbon & $\mathrm{mg} / \mathrm{L}$ & 0.14 & 0.21 & 0.00 & 0.04 & 0.00 & 0.25 & 0.13 & 0.20 & 0.15 & 0.23 \\
\hline
\end{tabular}

